\section{ar gor}

PPPL-2797

UC, $-420,426$
PREPARED FOR THE U.S. DEPARTMENT OF ENERGY, UNDER CONTRACT DE-AC02-76-CHO-3073

\title{
MEASUREMENTS OF CHARGED FUSION \\ PRODUCT DIFFUSION IN TFTR
}

\author{
BY
}

\author{
R.L. BOIVIN
}

December 1991
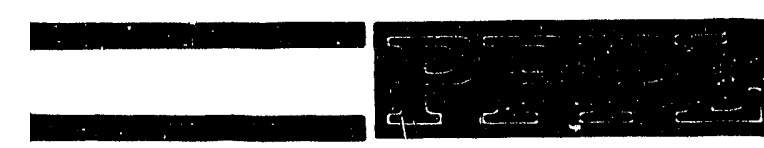

\section{maINGETON}

masma pargics

labogatogr

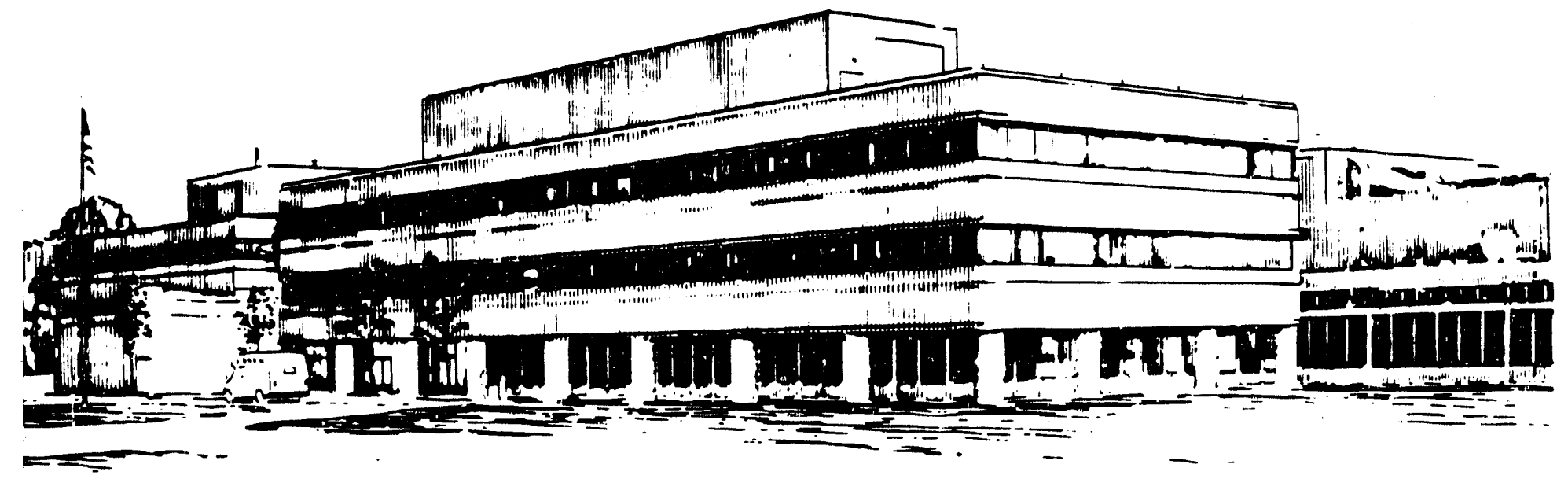




\section{NOTICE}

This report was prepared as an account of work sponsored by an agency of the United States Government. Neither the United States Government nor any agency thereof, nor any of their employees, makes any warranty, express or implied, or assumes any legal liability or responsibility for the accuracy, completeness, or usefulness of any information, apparatus, product, or process disclosed, or represents that its use would not infringe privately owned rights. Reference herein to any specific commercial produce, process, or service by trade name, trademark, manufacturer, or otherwise, does not necessarily constitute or imply its endorsement, recommendation, or favoring by the United States Government or any agency thereof. The views and opinions of authors expressed herein do not necessarily state or reflect those of the United States Government or any agency thereof.

\section{NOTICE}

This report has been reproduced directly from the best available copy.

Available to DOE and DOE contractors from the:

Office of Scientific and Technical Information

P.O. Box 62

Oak Ridge, TN 37831 ;

Prices available from (615) 576-8401.

Available to the public from the:

National Technical Information Service

U.S. Department of Commerce

5285 Port Royal Road

Springfield, Virginia 22164

$703-487-4650$ 
PPPL--2797

DE92 006815

\section{MEASUREMENTS \\ OF CHARGED FUSION PRODUCT \\ DIFFUSION IN TFTR}

Réjean Louis Boivin

October 1991 


\section{MEASUREMENTS \\ of Charged Fusion Product \\ DIFFUSION IN TFTR}




\title{
MEASUREMENTS \\ OF CHARGED FUSION PRODUCT \\ DIFFUSION IN TFTR
}

\section{Réjean Louis Boivin}

\author{
A Dissertation \\ presented to the \\ faculty of Princetor University \\ in candidacy for the degree \\ of Doctor of Philosophy
}

Recommended for acceptance by the

Depariment of

Astrophysical Sciences

October 1991 
The Goverment reserves for itself and others acting on its behalf a royalty free, nonexclusive, irrevocable, world-wide license for governmental purposes to publish, distribute, translate, duplicate, exhibit, and perform ary such data copyrighted by
the contractor.

\section{(c) 1991}

Réjean Louis Boivin

ALL RIGHTS RESERVED 


\section{A mes parents et frères; \\ Pour leur amour, et leur support}


. 


\section{Abstract}

The single particle confinement of charged fusion products, namely the $1 \mathrm{MeV}$ trition and the $3 \mathrm{MeV}$ proton, has been studied using a detector located near the outer midplane of TFTR. The detector, which measures the flux of escaping particles, is composed of a scintillator $[\mathrm{ZnS}(\mathrm{Ag})]$ and a system of collimating apertures, which permit pitch angle, energy and time resolution. It is mounted on a movable probe which can be inserted $25 \mathrm{~cm}$ into the vacuum vessel.

Measurements indicate a level of losses higher than expected from a first-orbit loss mechanism alone. The primary candidate for explaining the observed anomalous losses is the toroidal field ( $\mathrm{TF}$ ) stochastic ripple diffusion, theoretically discovered by Goldston, White and Boozer ${ }^{1}$. This loss mechanism is expected to be localized near the outer midplane where, at least at high current $(Z 1.0 \mathrm{MA})$, it would locally dominate over first-orbit losses. Calculations made with a mapping particle orbit code (MAPLOS) show a semi-quantitative agreement with the measurements. The predominant uncertainties in the numerical simulations were found to originate from the modeling of the first wall geometry and also from the assumed plasma current and source profiles. Direct measurements of the diffusion rate were performed by shadowing the detectur with a second movable probe used as an obstacle. The diffusion rate was also measured by moving the detector behind the radius of the RF limiters, located on the outer wall. Comparisons of these experimental results with numerical simulations, which include diffusive mechanisms, indicate a quantitative agreement with the TF stochastic ripple diffusion model.

${ }^{1}$ R. J. Goldston, R. B. White, and A. H. Boozer, Phys. Rev. Lett. 47, 647 (1981). 


\section{Contents}

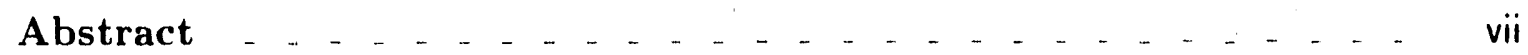

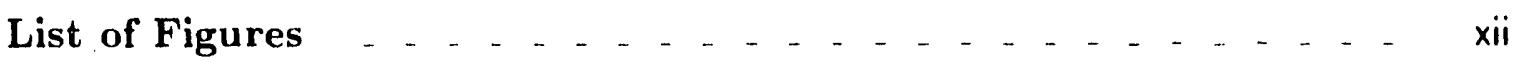

List of Tables … . . . . . . . . . .

Acknowledgments … . . . . . . . . . xix

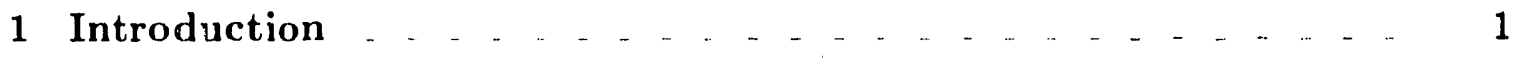

2 Previous Work _... . . . . . . . . . . . 5

2.1 Theoretical considerations _... . . . . . . . 5

2.1.1 Unperturbed orbits _... . . . . . . . 6

2.1 .2 Ripple trapping _. . . . . . . . . . . 10

2.1.3 Stochastic diffusion due to TF ripple _....... 13

2.1 .4 Turbulent diffusion _... . . . . . . . . 22

2.1.5 Other diffusion mechanisms _... . . . . . 22

2.2 Previous experimental results _ . . . . . . . . 23

2.2 .1 First-orbit losses _. . . . . . . . . . 25

2.2 .2 Toroidal field ripple losses _ . . . . . . . . 26

2.2.3 Turbulence-induced diffusion _. . . . . . . . . 27

2.2 .4 Other loss mechanisms _... . . . . . . . 28

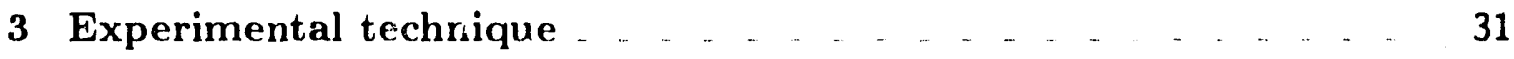


3.1 Detection system description _ _ _ . . . . . . . . . 32

3.1.1 Midplane probe _. _ _ _ _ _ . . . . . . 35

3.1.2 TFTR lost alpha detectors array _ _ _ . . . . . 45

3.1.3 Signal processing system _. . . . . . . . . . 45

3.2 Calibration _... . . . . . . . . . . . . 50

3.2 .1 The $\mathrm{ZnS}(\mathrm{Ag})$ scintillator _... . . . . . . . . . 50

3.2 .2 Optical calibration _. . . . . . . . . . . 63

3.2 .3 Supplementary diagnostics _ _ _ _ _ . . . . . 65

4 Characterization of CFP losses near the outer midplane _ _ _ 69

4.1 Theoretical expectations _ . . . . . . . . . . . . 70

4.2 Description of the experiments _ _ . . . . . . . . 84

4.3 Plasma current dependence _ _ _ _ . . . . . . . 85

4.3.1 Current dependence of the escaping flux _ _ _ _ _ _ 86

4.3.2 Pitch angle distribution _ _ . . . . . . . . . . 88

4.3.3 Poloidal distribution _ . . . . . . . . . . . 100

4.3.4 Absolute flux of CFPs _ _ _ _ _ _ _ _ . . . 102

4.3 .5 Energy distribution _. _ . . . . . . . . . . . . 105

4.4 Other experiments _ _ _ _ _ _ _ . . . . . . . . . 107

4.4.1 Current ramp experiment _. . . . . . . . . 109

4.4.2 Plasma compression _. . . . . . . . . . . 109

4.4.3 Beam power dependence _ _ . . . . . . . . . . 111

5 Direct measurements of diffusion _ _ _ _ _ _ _ _ _ _ _ 117

5.1 Preliminary observations _ _ . . . . . . . . . . 119

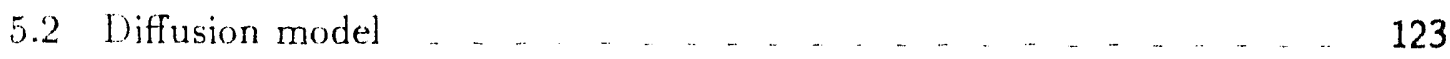

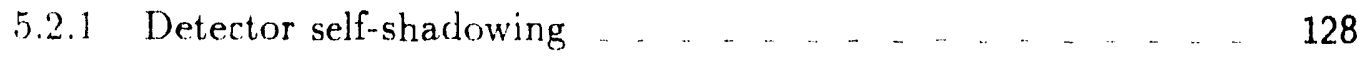

5.2 .2 Diffusion in the presence of a large obstacle _. . . . 130

5.2.3 Diffusion in the presence of a small obstacle _... . . . 133 
6 Other observed loss mechanisms _ _ _ _ . . . . . . . . 143

6.1 Neutral beam blips _. . . . . . . . . . . . 143

6.2 MHD-induced losses _. . . . . . . . . . . . . . 144

6.3 Sawteeth-induced losses _ _ . . . . . . . . . . 151

7 Suggestions for future work _ _ _ _ _ _ _ _ . . . . . 155

7.1 TF stochastic ripple diffusion _ _ . . . . . . . . 155

7.2 Ripple trapping _ _ . . . . . . . . . . . . . 156

7.3 MHD ripple-induced losses _ $\ldots \ldots \ldots$

7.4 ICRF high energy minority tail ions _ _ . . . . . . . . 160

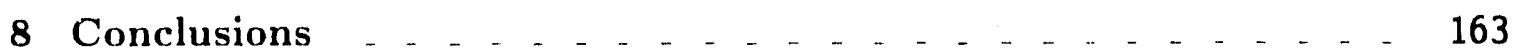

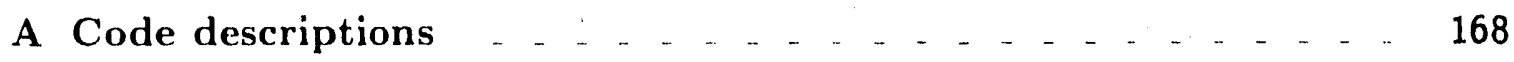

A.1 MAPLOS _. . . . . . . . . . . . . . . . . 168

A.2 Diffusion codes _. . . . . . . . . . . . . . . 173

A.2.1 Diffusion in presence of a small obstacle _ _ _ _ _ _ 173

A.2.2 Diffusion in presence of a large obstacle _ . . . . . $17 \mathrm{C}$

A.3 ORBIT code _. . . . . . . . . . . . . . . . 176

B Calibration details _... . . . . . . . . . . . 180

B.1 Calibration instrumentation _... _ . . . . . . . . 180

B.2 Beam parameters _. . . . _ . . . . . . . . . . . 181

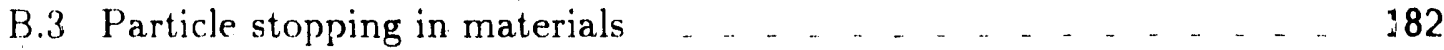

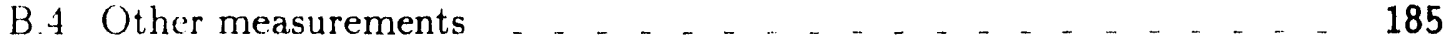

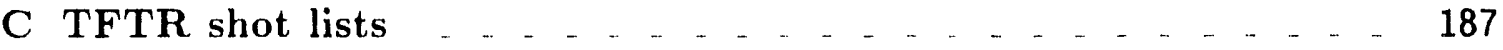

Bibliography _. . . . . . . . . . . . . . . . . . . . . . . 193 


\section{Figures}

1.1 Fusion reacíions cross-sections _. . . . . . . . . . . . . . 2

2.1 System of coordinates in toroid

2.2 Charged Fusion Product orbits _ _ _ . . . . . . . . . . . 9

2.3 Mapping of the particle classes _ . . . . . . . . . . . . . . 11

2.4 Field amplitude along a field line _. . . . . . . . . . . . 12

2.5 Ripple trapping region for TFTR _ _ _ . . . . . . . . . 14

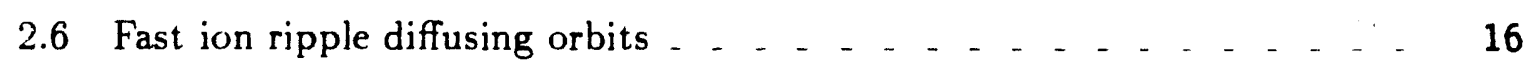

2.7 Poincaré plot of a CFP orbit in phase space _ _ _ _ _ _ _ _ 18

2.8 TFTR vacuum toroidal field ripple _ . . . . . . . . . . . . 19

2.9 Ripple loss domains _. . . . . . . . . . . . 20

2.10 Diagram of the impact location of a ripple lost particle $\quad 21$

2.11 Diagram of the MHD and pitch angle loss processes _ _ . . . 24

3.1 Detector's basic design _... . . . . . . . . . . 33

3.2 Location of detectors in TFTK _ . . . . . . . . . . 34

3.3 Midplane probe picture 36

3.4 Probe head picture _... . . . . . . . . . . . 37

3.5 First-orbit confined orbits _ _ _ _ _ _ _ _ _ 38

3.6 Midplane probe layout _... . . . . . . . . 40

3.7 Detail of midplane detector _ _ _ _ _ _ . . . . . 41

3.8 Aperture system dimensions _ . . . . . . . . . 42

3.9 Scintillator impact mapping _. . . . . . . . . . 43 
3.10 Geometrical resolution of midplane detector _ . . . . . . 44

3.11 Typical first-orbit loss for the bottom detector _ . . . . . . 46

3.12 Diagram of light recording system _ . . . . . . . . 48

3.13 Camera view of the scintillator _ . . . . . . . . . 49

3.14 Scintillator electron energy levels _. . . . . . . . . 51

3.15 High energy particles ranges in $\mathrm{ZnS}(\mathrm{Ag}) \ldots \ldots . \ldots 54$

$3.16 \mathrm{ZnS}(\mathrm{Ag})$ temperature dependence _.......... . . . . . 55

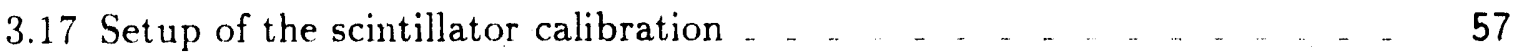

3.18 Pulse-height spectra from the scintillator calibration _.... 59

3.19 Scintillator efficiency vs. particle energy _ . . . . . . . 62

3.20 Angle of incidence dependence of light emissivity _ . . . . . 64

3.21 Detector optical resolution test _. . . . . . . . . 66

3.22 Photographic picture of the optical broadening _ . . . . . 67

3.23 Detector vignetting effect _ . . . . . . . . . . . . 68

4.1 Distribution of ripple diffusing particles in $\mu$ space _. . . . . . 72

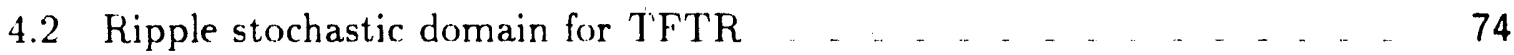

4.3 First-orbit confined trapped particles bounce point density _ . . . 75

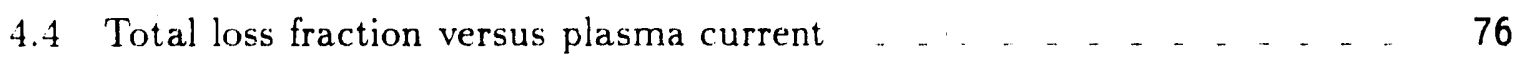

4.5 Vertical position dependence of the ripple step size _ _ f 78

4.6 Poloidal distribution of first wall impacts due to first-orbit and TF ripple losses _... . . . . . . . . . . . . . . 79

4.7 Confinement time distribution _... . . . . . . . 80

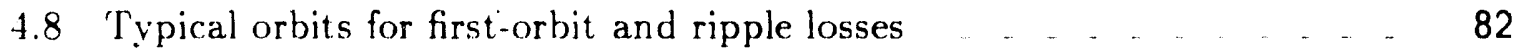

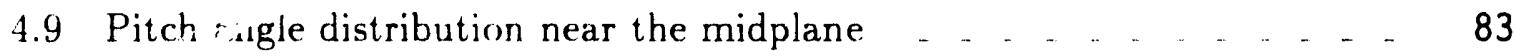

4.10 Neasured escaping flux current dependence _. . . . . . 87

4.11 Pitch angle distributions for current scan at large major radius _. 91

4.12 Fattest banana orbit variation with plasma current _..... 92

4.13 Pitch angle distributions for a "standard" plasma _. 94

4.14 Stochastic threshold scan _... . . . . . . . . 97 
4.15 Pitch angle distribution calculations with RF limiters _ _ _ . . 99

4.16 Variation of the pitch angle distribution during a partial detector radial scan

4.17 Poloidal distribution of lost CFPs _ . . . . . . . . . . . 104

4.18 Gyroradius distribution comparison _. . . . . . . . . . . . . 108

4.19 Current ramp experiment _. . . . . . . . . . . . . . . 110

4.20 Time dependence of the escaping flux during a plasma compression _ - 112

421 Neutral beam power dependence of CFP flux _ . . . . . . . . . 113

\$22 Time dependence of detected flux versus neutral beam power . _ . _ 115

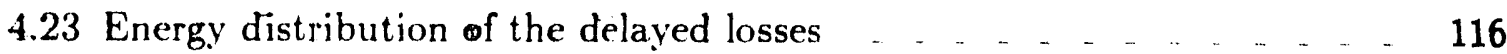

5.1 Aperture radial scan, $I_{p}=1.6 \mathrm{MA} \ldots \ldots \ldots$

5.2 Aperture radial scan comparison _.. . . . . . . . . . . . . . 121

5.3 Complete aperture radial scan, $I_{p}=1.15 \mathrm{MA} \ldots \ldots 122$

5.4 Experimental results of the probe shadowing experiment _ _ _ _ 124

5.5 Probe shadowing caleutation _ _ _ _ _ _ _ _ _ 126

5.6 Physical picture of the detector self-shadowing effect _ _ _ _ _ 129

5.7 Physical picture of the shadowing experiment with the RF limiters _ 132

5.8 RF limiters shadowing experiment with theoretical models _... _. _ 134

5.9 Shadowing experiment physical picture _... . . . . . . . . 136

5.10 Comparison of model with shadowing experiment results _ _ _ 138

5.11 Changes in the pitch angle distribution with the obstacle position _.. 141

6.1 Measured flux of (FPs during NBI blips _. 145

6.2 MHD-induced losses of CFPs during fishbone activity $\quad \ldots$

6.3 MHD finduced losses of CFPs during internal kink activity 147

6.4 Pitch 149

6.5 "Time dependence for two different pitch angles during MHD activity - 150

6.6 Burst of, escaping CFPs at the midplane detector during a sawtooth crash 152

6.7 Sawteeth modulated CFP losses at the midplane detector _ . . . . 153 
7.1 Conceptual design for a ripple-trapped particle detector _ _ _ _ _ 158

7.2 RF minority tail ions flux to detector during sarwtooth crashes _ _ . _ 161

A.1 Midplane vacuum TF ripple and numerical fit _ _ _ . . . . 171

A.2 MAPLOS algorithm _ . . . . . . . . . . . . . . 172

A.3 Poloidal position of TFTR probes and limiters _ _ . . . . . . 174

A.4 Block diagram for the small obstacle diffusion code _ _ _ _ _ _ _ 175

A.5 Block diagram for the large obstacle (RF limiters) code _ _ _ _ _ 177

A.6 Poloidal projection of an orbit at very low current _ . . . . . . 179

B.1 CFP energy loss through a $3 \mu \mathrm{m} \mathrm{Al} \mathrm{foil} \mathrm{\ldots} \mathrm{.} \mathrm{.} \mathrm{.} \mathrm{.} \mathrm{.} \mathrm{.} \mathrm{.} \mathrm{.} 184$

B.2 Setup for front to back comparison of the light emission of the scintillator 186 


\section{Tables}

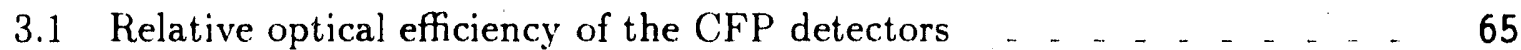

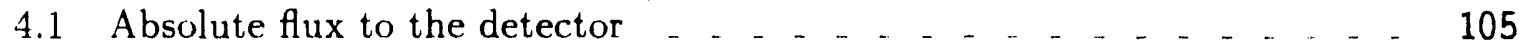

C.1 Shot list, current scan with large plasma _ _ _ . . . . . . . 187

C.2 Shot list, current scan with standard plasma _ _ . . . . . . . . 188

C.3 Shot list, power scan _ _ . . . . . . . . . . . . . . 189

C.4 Shot list, shadowing experiment with second probe _ _ _ _ _ _ 190

C.5 Shot list, shadowing experiment with RF limiters _ _ _ _ _ _ _ 191

C.6 Shot list, complete aperture radial scan with RF limiters _ _ _ _ . 192 


\section{Acknowledgments}

When I first considered doing my thesis work on alpha particle physics on TFTR, I was warned of the "evil" that comes with a big machine and its reputed student unfriendly environment. Looking back upon the two and an half years of my thesis work, I realize the opposite, that is how much great support I received, and how so many good people were involved in this work.

Great support indeed I received from my advisor, Stewart Zweben, who believed in me from the start, and never stopped encouraging me to do better. He taught me not only a great deal about alpha particle physics but also how to become a real, professional physicist. For his continuous support and for his dedication, I thank him very much. He deserves to be in the PPPL advisor's hall of fame, especially for his dedication to his students.

Substantial, but too often passed unnoticed, is the support that I received from Jim Strachan. He contributed greatly to the success of this research. And all this would not have been possible also, without the support, sometimes material, sometimes "political", from K. Young, D. Meade, R. Hawryluk, K. McGuire, S. Cohen, R. Goldston, D. Manos and L. Johnson. They all believed in the worthiness of my work and in my capabilities, and they gave me the opportunity to contribute to TFTR research efforts.

The heip was also important in the design and fabrication of the midplane probe. Steve Kilpatrick was always available for questions and problems with probes, and most important he made the cohabitation of probes easy. Bob Parsells, with his good sense of humour, made the fabrication and installation phases a bit less frustrating. And I wish to thank Tom Deverell for his help, and for sharing his vast machining expertise. Thanks also to D. Palladino and K. Owens for their precious contributions.

But once the probe was fabricated, it had to be installed and that's where Tim 
Bennett proved to be always available and dedicated to the cause. Precious help I obtained also, on many aspects of the probe and data acquisition systems, from S. Hayes, P. Sichta, G. Lemunyan, G. Barnes, B. Bergin and J. Montague.

But once it is installed, we have to know what the data means! L. Roquemore was always willing and cooperative when I needed the ion beam working during the calibration of the detectors. The contributions of R. Motley (who also lent the two precious surface barrier detectors), Z. Lin, R. Shoemaker and T. Holoman were also invaluable.

But then, we want to make sense of all that data, we want to get some physics out of this. The tielp that I received from Roscoe White, Steve Scott and Rob Goldston was important in the understanding of ripple transport. R. White, S. Scott and S. Zweben did also a tremendous job as readers, in helping clarifying my ideas and correcting my Québécois English. However, any remaining sacrilege to the language of Shakespeare is solely my responsibility. I would like to thank also G. Hammett, C. Barnes, M. Tuszewsky, C. Bush and Eric Fredrickson for their contributions. I was also very lucky to profit from the vast computer expertise and dedication of $M$. Diesso and J. Felt. My colleagues R. Duvall and D. Ward were very helpful, especially in dealing with the fast but often "dumb" CRAY computers. And to the TFTR group thank you for your help and for this great research opportunity.

Special thanks to FMI Inc. who gave us the precious material for the fabrication of the probe's armor and to the Natural Sciences and Engineering Research Council of Canada (NSERC) who supported for four years my stay in Princeton. This work has been done under U.S. D.o.E. contract No. DE-AC02-76-CH03073.

Thanks also to $S$. Jardin who as my second year advisor, was very supportive Many thanks to B. Sarfaty, D. Larsen and B. Carey who acted as the good Godmothers and helped in softening many rough edges of Princeton life.

Finally, but nonetheless, I had the fantastic opportunity of meeting and discovering some very nice and special people here in Princeton. Among them my classmates, Steve Anderson, C. Forest, Y.-S. Hwang, S. McCauley, D. Moore, Ron Radeztsky and Pat Simon and also John Cuthbertson and Jeff Dooling. Roger Beckie with his sharp (and always amusing) view of the society, enriched my Princeton's experience in many aspects, and helped me in keeping a more balanced way of life. Special thanks also to the Troiano's family for their continuous care. 
Finally, I owe much to the two Dudes, Kevin Ilcisin (including his still growing family) and Don Roberts, who both later proved to be visionaries (of my future), although they probably didn't have a perfect timing! I shared with them my most difficult and nost exciting times. I tried to give them in return as much morale support as they gave me earlier. I was sad to see my friends leaving for the West Coast (Yes! go West young man, truer than ever!) and I hope that none of our friendship will fade with distance.

Merci à tous et à toutes!

Réjean Boivin

Princeton, NJ

August, 1991 
It was the best of times, it w's the worst of times, it was the age of wisdom, it was the age of foolishness, it was the epoch of belief, it was the epoch of incredulity, it was the scason of Light, it was the secuson of Darkiness, it was the spring of hope, it was the uinter of despair, we had cverything before us, we had nothing before us, we were all going to Heaven, we were all going the other way...

Charles Dickens, A Tale of Two Cities, Bk.i, ch.1 


\section{Chapter 1}

\section{Introduction}

The prospect of an abundant and "clean" source of energy from thermonuclear fusion has been the focus of research for scientists for more than 30 years now. However, the process of producing energy from a self-sustained reaction must first go through an initial phase in which energy is injected. In the case of tokamak technology this is predominantly done by the injection of fast neutrals (Neutral Beam Injection, NBI) or of radio-wave energy (Radio Frequency, RF). Existing research tokamaks are still at this level, where large amounts of power $(\leqslant 30 \mathrm{MW})$ are injected and where only a small amount of fusion power ( $\lesssim 60 \mathrm{~kW}$ for NBI and $150 \mathrm{~kW}$ for $\mathrm{RF}$ ) is obtained in return.

One of the main reasons for such a low return can be seen in Fig. 1.1. In present day experiments, the fuel that is used consists only of deuterium (and sometimes of deuterium and ${ }^{3} \mathrm{He}$ ) but never of deuterium and tritium. Tritium is an unstable element and needs special handling capabilities. It has also been avoided so far because the $\mathrm{D}$ - $\mathrm{T}$ fusion reaction produces many more neutrons which would rapidly activate the tokamak. However, this reaction has the very big advantage of a much higher cross-section, and so the mix of deuterium with tritium is the envisioned fuel for fusion reactors. The other important feature of this fusion reaction comes from the production of the $3.5 \mathrm{MeV}$ alpha particle which, because of its charged state, could be confined by the poloidal and toroidal fields of the tokamak. In fact, this confinement is necessary because the alpha particle energy would serve to sustain the high temperature needed for the fusion reaction. When the power deposited by the alphas reaches the plasma energy loss rate the reaction reaches ignition ${ }^{1}$ and consequently, auxiliary heating would be no longer needed. 


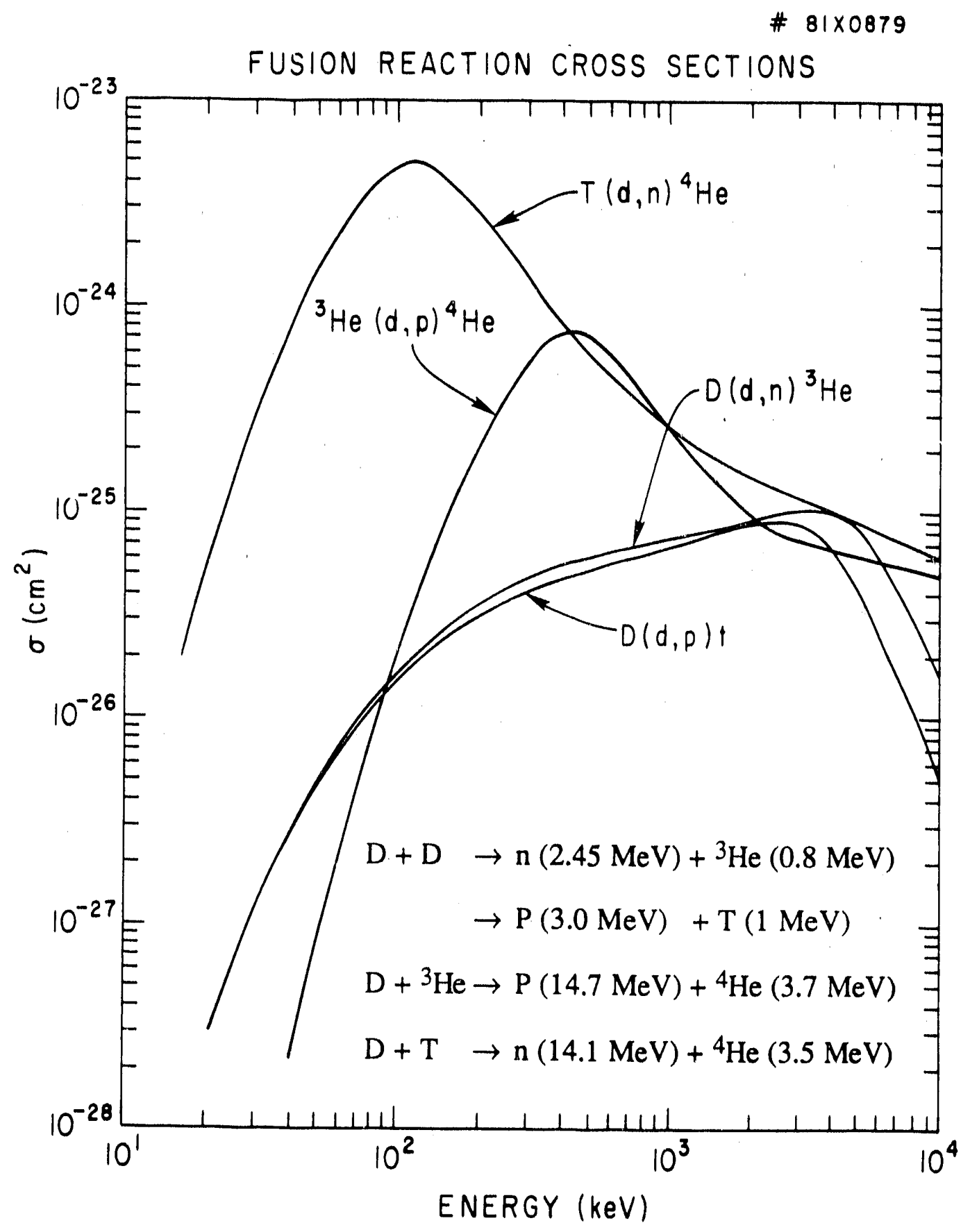

Figure 1.1: Cross-sections for the three elementary fusion reactions with their fusion products. 
Since the confinement of alpha particles is crucial, it is very important to understand and to eliminate any loss mechanism. Unfortunately, the direct experimental study of alpha particle heating physics has been hampered mainly because D-'I' fuels have not yet been used. However, it is possible to use the Charged Fusion Products (CFP) generated in the other fusion reactions, like the $1 \mathrm{MeV}$ triton, $3 \mathrm{MeV}$ proton, $0.8 \mathrm{MeV}{ }^{3} \mathrm{He}, 3.7 \mathrm{MeV}$ alpha and the $14.7 \mathrm{MeV}$ proton, in order to simulate the phy'sics of the $3.5 \mathrm{MeV}$ alpha particle.

On the other hand much work has been done on theoretical aspects. For example, it was discovered in $1981^{2}$ that the presence of periodic non uniformities (ripples) in the toroidal magnetic field could lead to the stochastic diffusion of high energy particles (e.g. CFPs). This loss mechanism, believed to be observable only towards the outer midplane of the tokamak, ${ }^{3}$ has, until now, never been the object of active experimental research. This subject regained importance when projections indicated that the alpha particles losses on the first wall of tokamaks would be highly localized. ${ }^{4}$ This could mean that, even with a globally small amount of losses (less than a few percent, negligible when trying to reach ignition), the localization of wall impacts could create wall heat load problems, as in BPX and ITER ${ }^{5,6}$ for example.

We report in this thesi- the results of experiments done on the Tokamak Fusion Test Reactor (TFTR) on the confinement of CFPs. These results were obtained by using a pitch angle (or equivalently, magnetic moment), energy and time resolved detector located just below the outer midplane, inside the first wall of TFTR. The detector was primarily designed to look at the effects of the toroidal field (TF) stochastic ripple diffusion on the confinement of trapped CFPs. Experimental results will be presented along with numerical simulations based on orbit following codes.

This thesis will be structured as follows. In Chapter 2 previous work both on theoretical and experimental aspects, will be reviewed. The emphasis will be on a review of work done on the subject of ripple transport, and specifically on TF stochastic ripple diffusion. In Chapter 3, we will examine the experimental technique, the detector, its characteristics and its calibration. In Chapter 4, experimental results from the midplane detector will be shown and will be compared to numerical simulations based on the theoretical model of TF ripple diffusion. ${ }^{2}$ Direct measurements of the trapped particle diffusion rates will be presented in Chapter 5, based on a technique involving obstacles (a probe or RF limiters) which shadowed the detector located behind it. Chapter 6 is a brief survey of other loss mechanisms that have been observed 
at the midplane with some possible explanations. Finally, in Chapters 7 and 8 , we will summarize and present avenues ior future research. 


\section{Chapter 2}

\section{Previous Work}

Since the beginning of fusion research, work has been done in the field of CFP physics, and more specifically on alpha particle confinement and alpha effects on plasma stability. The importance given to that topic is increasing steadily as we tokamak parameters approach reactor conditions. So far, much of the research effort has been devoted to theoretical aspects, since the experimental study of CFPs has been limited to a small number of experiments and machines. Theoretical considerations will be reviewed first, covering the principal sources of particle losses in tokamaks, followed by a review of experimental results.

\subsection{Theoretical considerations}

The internal structure of a tokamak can, because of its complexity, be the source of several loss mechanisms for CFPs. The first section concerns the basic magnetic (toroidal and poloidal) structure of a tokamak plasma and its associated ion drifts. These drifts are responsible for the most common and well understood loss process known as first-orbit losses (also called sometimes prompt losses simply because of the speed at which they occur). All the other diffusion or loss processes originate from orbit perturbations (from collisions or field perturbations) and would be superimposed on the first-orbit process. For CFPs, they are essentially perturbations of the magnetic field structure originating from an outside source like the toroidal field (TF) coil ripple (or also error fields) or from an inside source like magnetohydrodynamic (MHD) activity, coherent mode or sawteeth, or turbulence (of magnetic or electrostatic origin). 


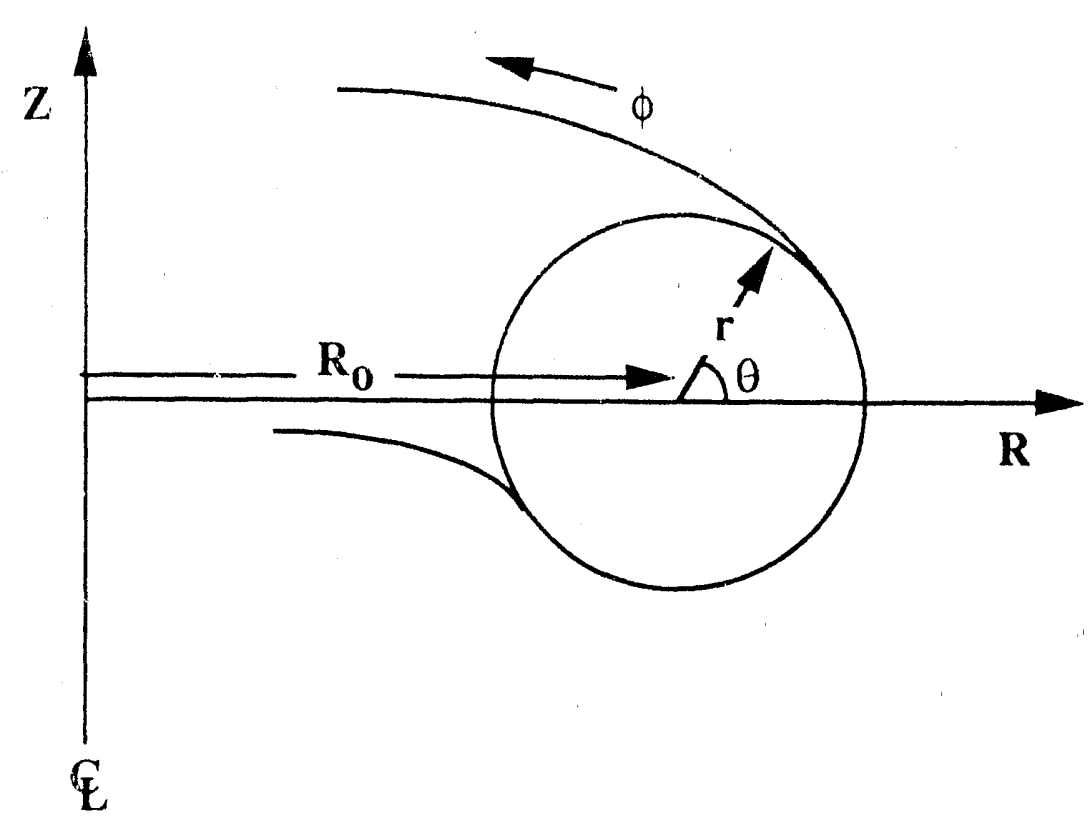

Figure 2.1: System of coordinates in toroidal geometry.

Before entering into the question of orbits and magnetic field it would be appropriate to describe the internal geometry of the tokamak and its associated variables. In Fig. 2.1 are shown the different coordinates. For the global geometry of the torus, we will use $\{R, Z, \phi\}$ which are respectively the major radius, the vertical position and the toroidal angle. In the frame of reference attached to the plasma we will use the variables $\{r, \theta\}$ which are respectively the minor radius and the poloidal angle. We also define the toroidal pitch angle $\chi$, as the angle of a particle trajectory with respect to the toroidal direction, i.e. $\cos \chi=\boldsymbol{v} \cdot \hat{\boldsymbol{\phi}} / \mathrm{v}$. By using this definition we neglect the poloidal field and so the parallel direction refers to the toroidal component only.

\subsubsection{Unperturbed orbits}

From a simple description of a plasma in terms of magnetic field and current distribution (poloidal magnetic field) one can recreate, at least numerically, the orbits of ions in order to study their confinement. For doing so, one can use the symmetries 
of a tokamak and the particularities of the energetic ions to simplify the problem. In this case three constants of motion can be found, the energy $E$, the magnetic moment $\mu \equiv m v_{\perp}^{2} / 2 B$, and the toroidal component of the canonical momentum $P_{\phi}=m v_{\|} R-q R A_{\phi}$, where $q$ is the particle charge and $A_{\phi}$ is the toroidal component of the vector potential. Each of them has a region of validity which is important to understand.

The conservation of the particle's energy can be "broken" in some cases. In the absence of electric fields, the particle's Hamiltonian is independent of time and its energy is thus conserved. However, when electric fields are present, externally applied or induced by RF heating or plasma compression, or at small scales, with the presence of collisions (e.g. slowing-down), the energy is no longer conserved. ${ }^{7}$ In the case of CFPs and for typical plasma conditions, large scale electric fields are negligible and the collision frequency is small (at least at their birth energy where the electron drag dominates), typically of the order of $1 \mathrm{sec}^{-1}$ for a $1 \mathrm{MeV}$ triton.

The magnetic moment is an adiabatic invariant and is the most better preserved constant of the three. It is conserved in the absence of sharp variations of the magnetic field in space $(\partial B / \partial x \ll B / \rho$, where $\rho$ is the gyroradius) or in time $(\partial B / \partial t \ll B \Omega$, where $\Omega$ is the gyrofrequency), as in normal discharges. It could also change during classical collisional pitch angle scattering but the associated frequency is considerably smaller than slowing down (by 4 orders of magnitude).

Finally, the canonical momentum $P_{\phi}$ is conserved only if the tokamak is axisymmetric, if the toroidal angle $\phi$ is an ignorable coordinate. In many tokamaks (e.g. TFTR) this is true as a first approximation, but not when we include details of the magnetic structure as we will see in section 2.1.3. However since the deviations from axisymmetry are usually small, perturbation techniques ${ }^{8}$ can be employed for deriving particle orbits.

In the case of an axisymmetric system, one can use the three constants of motion in order to find the particle location and velocity at any time. In practice and for more general cases one can integrate numerically the drift equations. The two major components are the curvature and the $\nabla B$ drifts which can be combined in a simple expression $^{9}$ :

$$
\boldsymbol{v}_{d r}=\frac{\mu}{q} \frac{B \times \nabla B}{B^{2}}+\frac{m v_{\|}^{2}}{q B^{2}} \frac{\boldsymbol{R}_{c} \times \boldsymbol{B}}{R_{c}^{2}}
$$


where

$$
\frac{\boldsymbol{R}_{c}}{R_{c}^{2}}=-(\hat{\mathbf{b}} \cdot \nabla) \hat{\mathbf{b}}
$$

where $\hat{\mathbf{b}}=\mathbf{B} / B$ and $q$ is the particle charge. Given a birth location, only the energy and magnetic moment are necessary. For energetic particles like CFPs, the drifts will bring them relatively far from their original flux surfaces for example at very low current ( $<0.5 \mathrm{MA}$ ) fast ions would simply drift in the ion $\nabla B$ direction which is down for the case of TFTR. The presence of a plasma current, a fundamental feature of the tokamak, serves to confine charged particles through the rotational transform by which field lines are twisted. In the cylindrical approximation this field line twist can be quantified by the value $q(r)=r B_{o} / R_{o} B_{\theta}$, also called the safety factor for stability reasons. The effect of the plasma current is slightly different whether the particle moves along (co-going) or opposite (counter-going) to the plasma current (toroidal field is antiparallel to the plasma current in TFTR). In the case of a cogoing (counter-going) particle, the ion will drift inwards (outwards), with respect to the major radius direction, above the midplane and outwards (inwards) below the midplane (see Fig. 2.2). For a given midplane crossing point (i.e. a given $P_{\phi}$ ) the poloidal projection of a particle orbit is thus determined by two constants of motion, the energy and the magnetic moment.

Furthermore, the orbits can be grouped in two categories; trapped or passing. In the case of passing particles, the orbit is quite simple, circulating monotonically in one toroidal direction. Projected in the poloidal plane the urbit has a non-circular shape usually wrapped around the magnetic axis (see Fig. 2.2). In the other case, the parallel component of the particle velocity is not large enough to allow the particle to pass over the magnetic hill created by the radially (major radius direction) decreasing magnetic field. At some point in the orbit all the kinetic energy of the particle is concentrated in its gyromotion (assuming the magnetic moment is constant). The translation motion of the particle thus stops and then reverses direction. The particle becomes "trapped" in the rnagnetic field and will bounce each time that $v_{\|}=0$. A third category of particle orbits can be found at low plasma current and are called pinch orbits as shown in Fig. 2.2. A complete description of fast ions orbits can be found in the literature. ${ }^{10,11}$

For a particle of given energy we can represent any guiding center orbit in the poloidal plane of a tokamak by using its magnetic moment and its radial crossing 

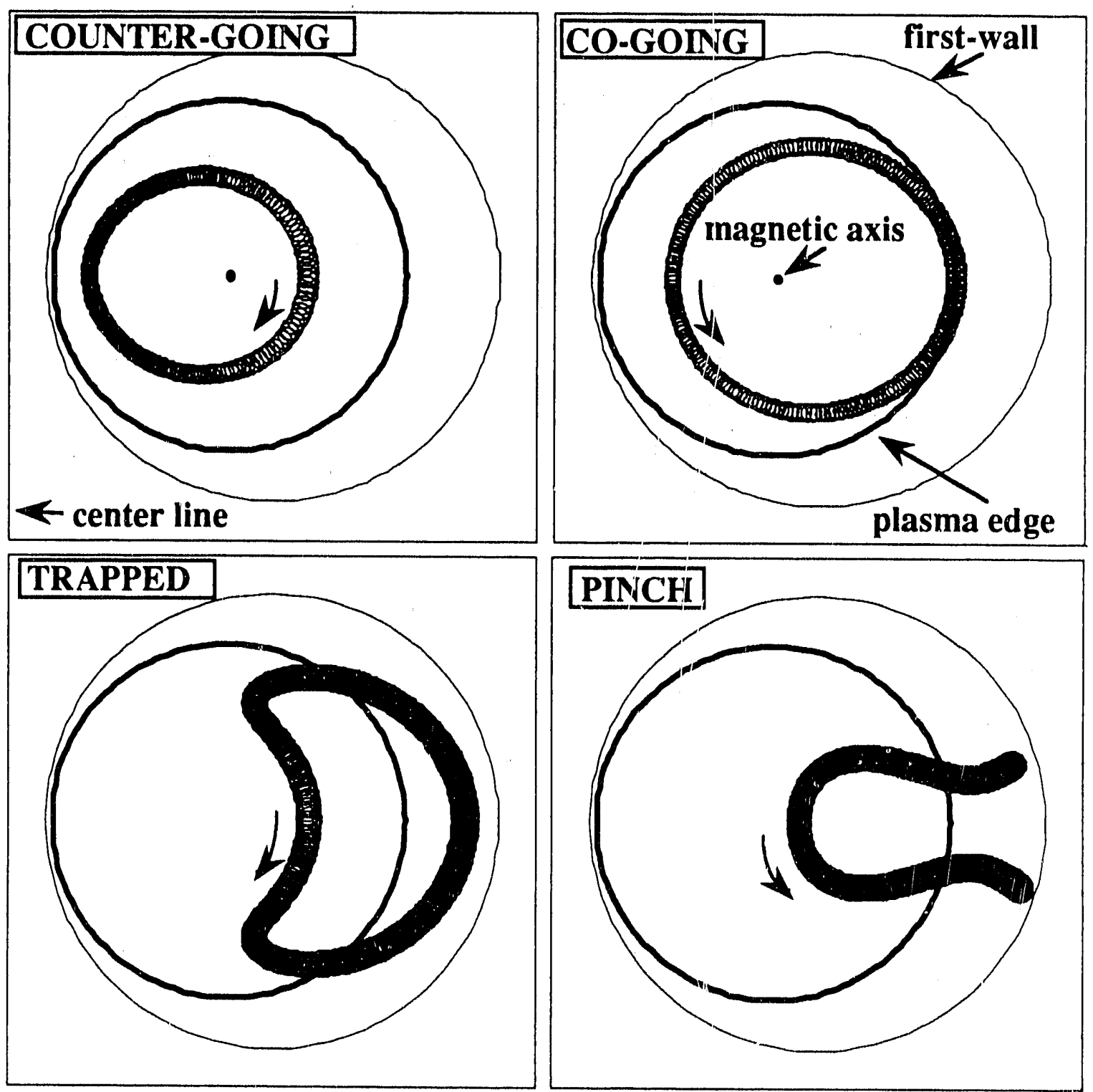

Figure 2.2: Typical orbits for Chorged Fusion Products. On top, counter-going (left) and co-going (right) passing particles, and at the bottom, trapped (left) and pinched (right) orbits. 
point at the outer midplane. In Fig. 2.3 is shown the distribution of counter-going particles as a function of their magnetic moment (normalized by $\mu_{o} \equiv E_{o} / B_{o}$, where $E_{o}$ is the birth energy and $B_{o}$ is the field amplitude at the magnetic axis) and outer midplane crossing point (a similar diagram can be obtained for the co-going particles, on which we use the inner midplane crossing point instead). The diagram also shows the separation between passing particles (lower left) and trapped particles (upper right) to which we will refer as the passing-trapped boundary. When the particle drift becomes too large, i.e. when the distance from the the birth flux surface becomes on the order of half the minor radius, particles will hit the wall (on the lower half of the vacuum vessel in the case of TFTR) on their first poloidal orbit, leading to first-orbit losses. For energetic particles like CFPs and at low current (typically less. than 0.6 MA for TFTR) those losses can be quite large (i.e. $>50 \%$ ). Also shown are the points $\mathbf{I}$ and $\mathbf{O}$ where barely trapped particles hit exactly at the inner and outer midplane wall respectively. This diagram will be used in other sections for illustrating other diffusion mechanisms and loss cones.

This one-to-one correspondence between particle's parameters (magnetic moment, energy, birth location) and its orbit can be used, at least theoretically, to infer the source and current profiles from loss measurements at the wall. Some work has been done on this subject both theoretically ${ }^{12,13}$ and experimentally. ${ }^{14}$ This technique has some drawbacks, it needs a relatively large number of detectors and a very good resolution in energy and magnetic moment. This technique also necessitates low plasma current so that losses would be dominated by first-orbit losses. At higher current, CFP losses could originate from many different mechanisms and might prevent such an analysis. It is also important to realize that at high current, no first orbit reaches the wall from the plasma center and consequently, that region would be "out of view" (by a first-orbit process). Neutron measurements are certain'ly easier to make for determining the source profile except for the study of $\mathrm{D}-{ }^{3} \mathrm{He}$ reaction profile.

\subsubsection{Ripple trapping}

One of the main features of the tokamak is its toroidal symmetry (also known as axisymmetry). Ideally, any toroidal position (any toroidal angle $\phi$ ) would be indistinguishable from another. Unfortunately, in practice, such precision in toroidal symmetry can only be approached. For example, the much needed access to the 


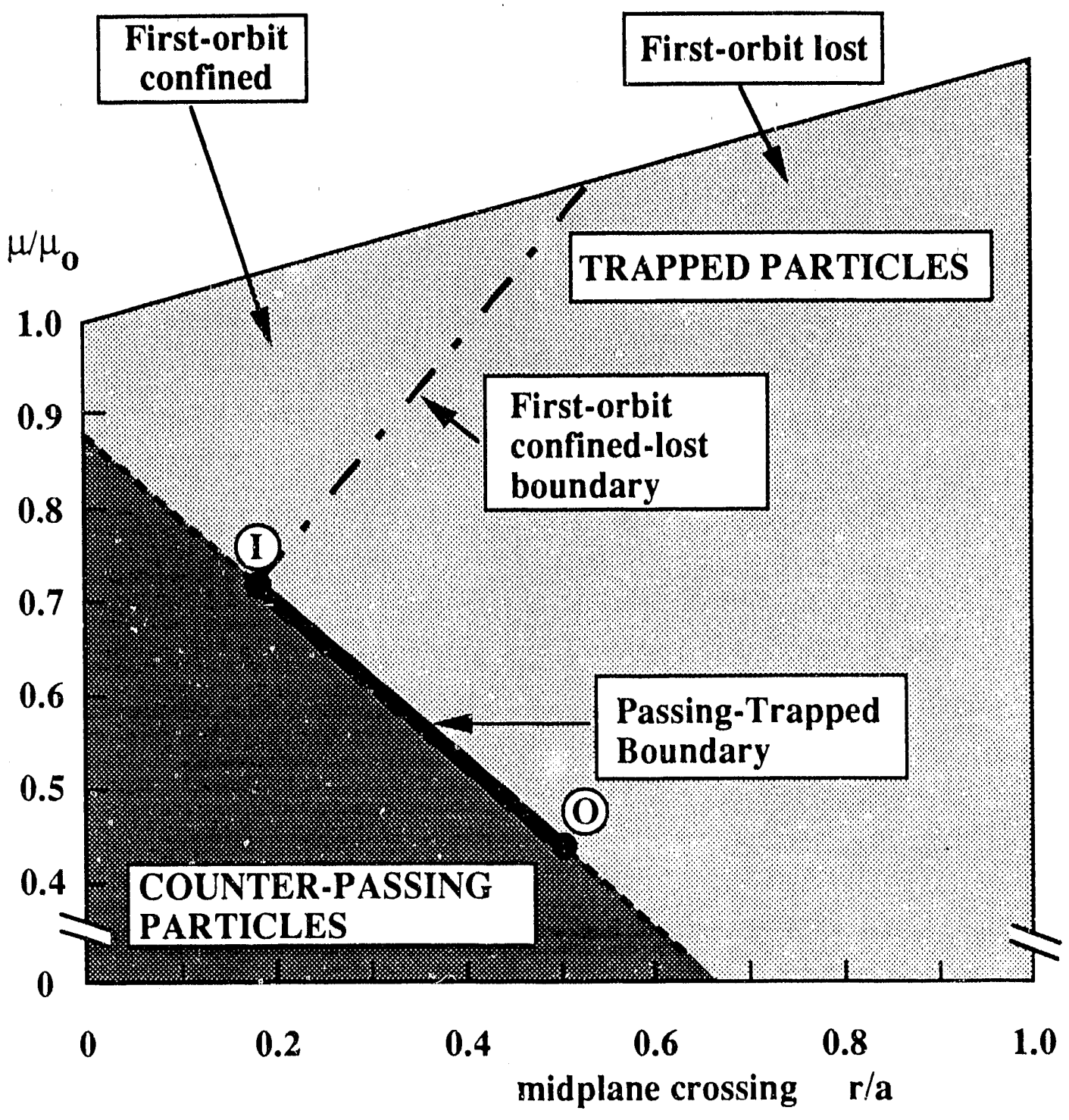

Figure 2.3: Distribution of the different orbit classes in $\mu$ space versus their outer midplane crossing point, for the case of counter-going particles. 


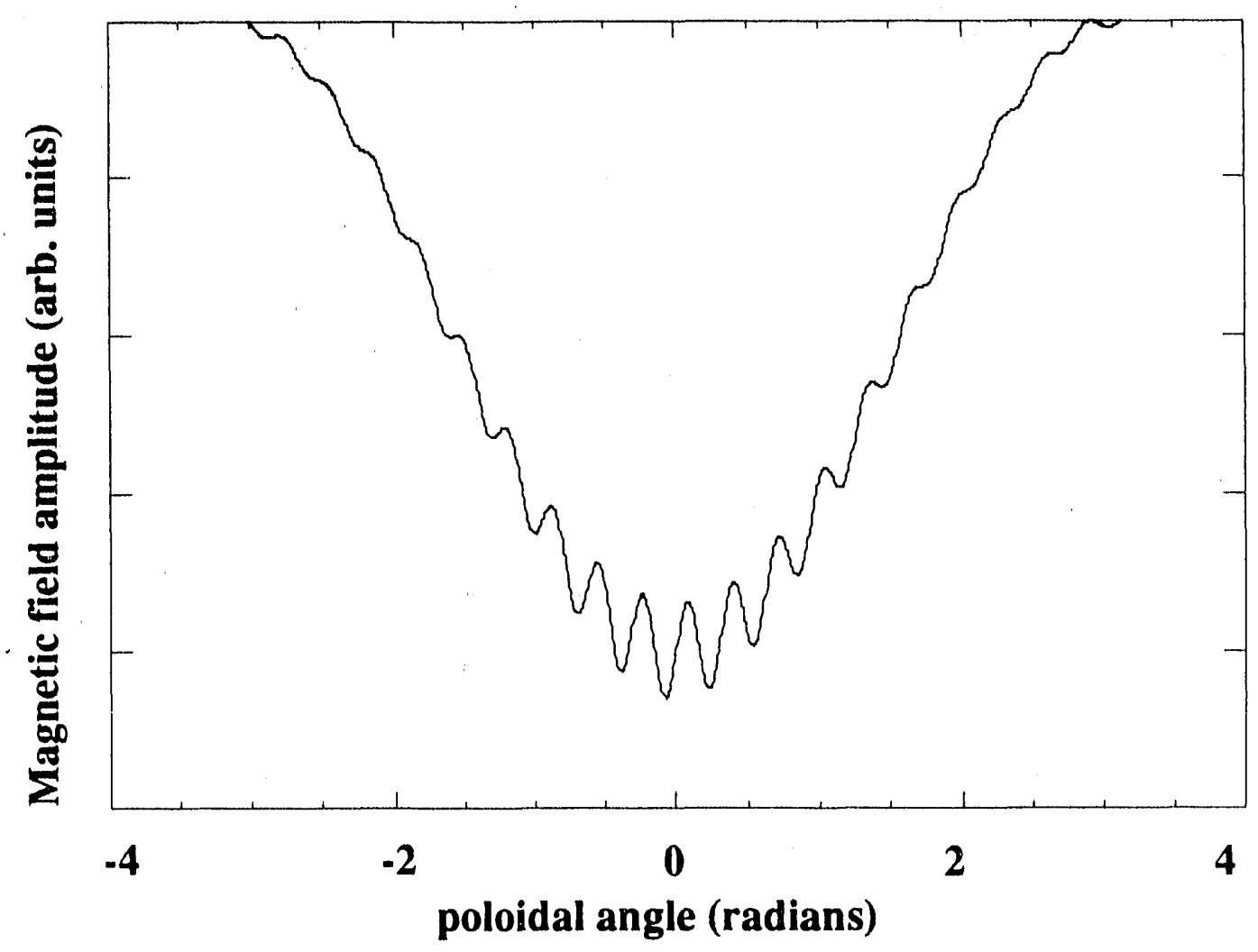

Figure 2.4: Magnetic field amplitude along a field line with the presence of ripples.

plasma for diagnostics and auxiliary heating create gaps between field coils. The toroidal field coils are limited in number and in toroidal extension creating periodic toroidal distortions in the magnetic field. This distortion is known as the Toroidal Field ( $\mathrm{TF}$ ) ripple and has been studied since the tokamak has been introduced, and has been recognized since then as a potential problem for single-particle confinement.

In the case where the ripples are a small perturbation we can express the magnetic field strength as being simply (in the large aspect ratio approximation):

$$
B=B_{o}\left(1-\frac{r}{R_{o}} \cos \theta+\delta(r, \theta) \sin (N \phi)\right)
$$

where $N$ is the number of toroidal field coils and $\delta \equiv\left(B_{\max }-B_{\min }\right) /\left(B_{\max }+B_{\min }\right)$ is the ripple amplitude (average to peak value). 
The first identified effect of TF ripples is the possible creation of secondary magnetic wells ${ }^{15}, 16,17$ especially near the outer wall of the tokamak, in which particles can become trapped. Those particles execute orbits known as superbananas, and would be lost quickly to the wall because the toroidal drift is not compensated by the rotational transform. In the axisymmetric case, the magnetic field strength varies monotonically as $1 / \mathrm{R}$ as it goes from the inner region to the outer wall. In the presence of ripples the field lines exhibit local minima and maxima as shown on Fig. 2.4 . Those local extrema ${ }^{18}$ exist, i.e. when the ripple is large enough, when

$$
\alpha^{\star} \equiv \frac{\epsilon|\sin \theta|}{N q \delta}<1
$$

where $\epsilon$ is the local aspect ratio, $\mathrm{N}$ the number of coils, $\theta$ is the poloidal angle and $\delta$ the local ripple amplitude. Any trapped particle having its bounce point in or very near this region (see Fig. 2.5, which shows the calculated ripple trapping region for a TFTR $I_{p}=1.4 \mathrm{MA}, B_{T}=4 \mathrm{~T}$ and $R_{o}=2.60 \mathrm{~m}$ case) can be trapped in the ripple well. Goldston and Towner ${ }^{18}$ also derived the trapping rate as a function of the particle and plasma parameters.

The trapping of CFPs can occur in both collisional and collisionless regimes. Collisions can induce slight changes (i.e. by pitch angle scattering, which changes $\mu$ ) in their orbits bringing them in the ripple well. However, in the collisionless case, the trapping originates from the non-uniformity of the ripple across the cross-section of the plasma. Trapped particles would miss the local magnetic hill on the inner part of their banana orbit but not necessarily on the outer one, thus becoming ripple trapped.

It is clear from the example shown in Fig. 2.5 that ripple trapped losses of CFPs is expected to be quite small in TFTR. Very few trapped particles have their bounce points in the trapping region, in fact we will see in Fig. 4.3 that the bounce points density is maximum inside the major radius of the magnetic axis. However this could be different for ICRF heated discharges and a method for looking at ripple trapped losses is discussed in Sect. 7.2 .

\subsubsection{Stochastic diffusion due to TF ripple}

The modification of the magnetic field structure due to the TF ripple can also lead to additional drifts for particles. When computing drift orbits for the ions in an axisymmetric tokamak the poloidal projections of the orbits close on themselves 


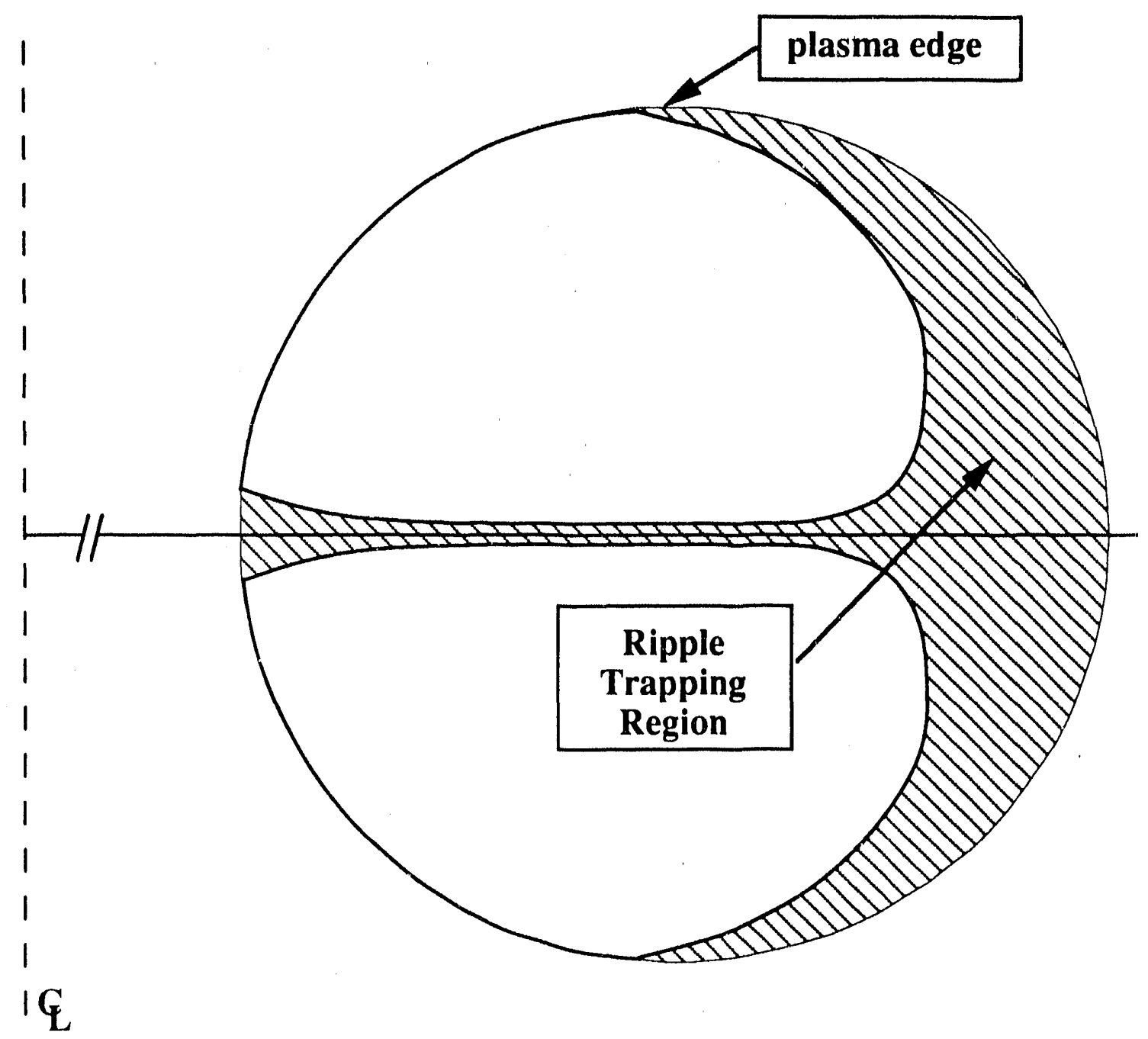

Figure 2.5: Typical ripple trapping domain for TFTR in a $I_{p}=1.4 M A, B_{T}=4 T$, $R_{\mathrm{o}}=2.60 \mathrm{~m}$ discharge. 
after one poloidal transit. When one includes the perturbations due to the ripple, additional drifts will emerge. Because the perturbation is periodic (typically like $\sin (N \phi)$ where $N$ is the number of coils), the drift accumulated over a complete ripple period will be averaged out. The only place where the drift would be uncompensated for is at the bounce point of trapped particles, explaining why passing particles are unaffected by the TF ripple. ${ }^{8}$ One can evaluate the amplitude of this drift by using a perturbative method. ${ }^{8}$ The small perturbation in the equations of motion due to the ripple is integrated along an unperturbed orbit giving explicitly the change in flux surface for the particle bounce point. This change of flux surface corresponds to a radial step size of:

$$
\Delta r \simeq\left(\frac{N \pi}{\sin \theta_{b} \mid}\right)^{\frac{1}{2}}\left(\frac{q}{\epsilon}\right)^{\frac{3}{2}} \rho \delta \sin \left(N \phi_{b}\right)
$$

where $\rho$ is the gyroradius and all the variables (including the poloidal and toroidal angles $(\theta$ and $\phi)$ ) being evaluated at the bounce point.

With the presence of the $\sin \left(N \phi_{b}\right)$ factor in the expression for $\Delta r$, the process is not diffusive by itself. At this point, an additional mechanism is necessary to decouple the different radial steps to create a diffusive process. This can be done by two very different actions, the first, by the effects of collisions (the banana drift or the ripple plateau regime) and the second by the effect of toroidal drift (the TF stochastic ripple diffusion).

In the case where collisions are dominant, ${ }^{18}$ they induc: changes in the magnetic moment of trapped particles and consequently the radial ripple excursions will be decorrelated. The bounce point would move diffusively as show'n in Fig. 2.6. ${ }^{18}$ This mechanism is important when the collision frequency is similar to the bounce frequency $\left(\nu_{\text {collision }} \sim \omega_{\text {bounce }} \equiv 1 / \tau_{k}\right)$. Particles are eventually lost to the first wall either by a direct hit or by ripple trapping. If the collisions are frequent $\left(\nu_{\text {collision }} \gtrsim \omega_{\text {bounce }}\right)$ particles will diffuse at the ripple plateau rate $D \sim(\Delta r)^{2} / \tau_{b}$. On the other hand, if the collisions are not that frequent $\left(\nu_{\text {collision }} \leqslant \omega_{\text {bounce }}\right)$ they will diffuse at the banana drift rate $D \sim(\Delta r)^{2} \nu_{\text {collision. }}$. Both of these collisional processes would be more important for fast ions (i.e. fast neutral beam ions) than for (FPs at their birth energy (for which collisions are relatively infrequent).

The second case, namely the TF stochastic ripple diffusion, is a purely collisionless process. The radial step is mainly vertical (to be exact, along $|B|$ surfaces), $\mu$ being 


\section{Banana Drift \\ Regime

\author{
TF Stochastic
} \\ Ripple Diffusion}

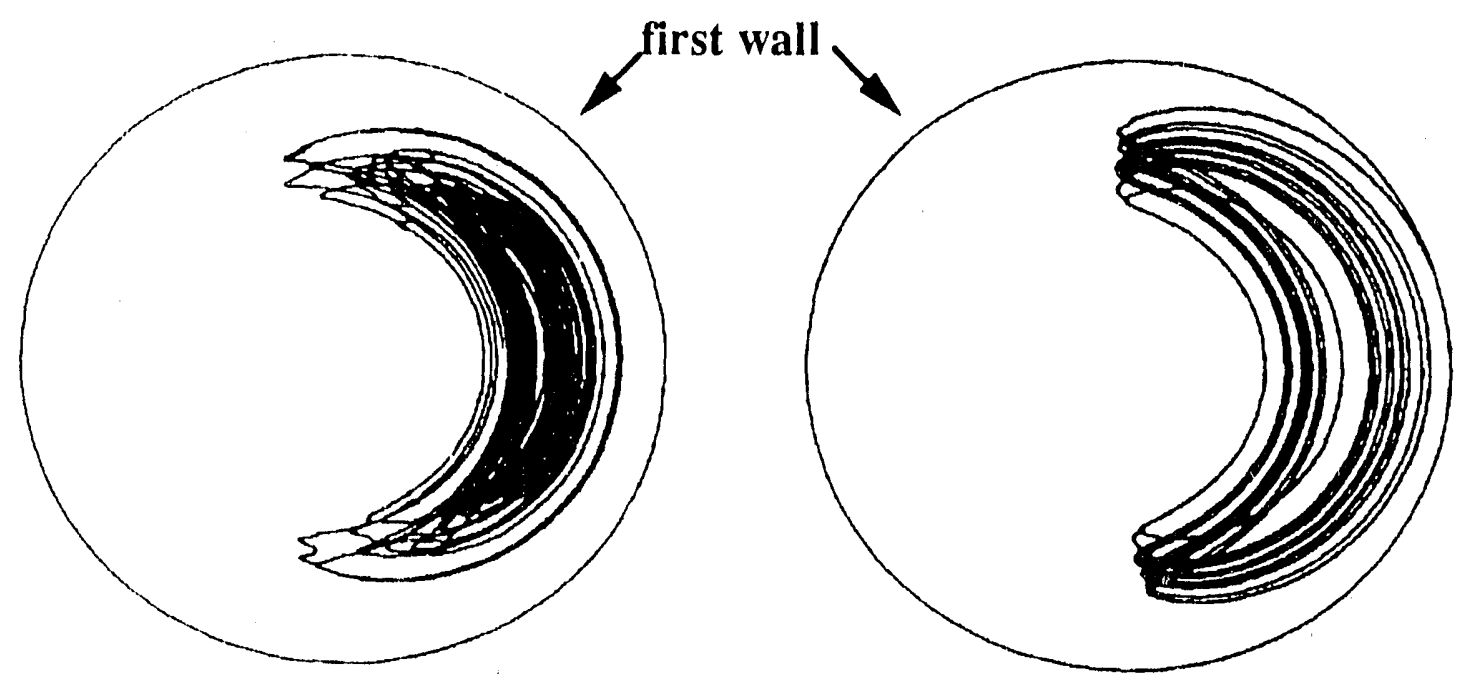

Figure 2.6: Typical orbits for a fast ion escaping by ripple diffusion (Goldston and Toume ${ }^{18}$ ). (On the left, collisions induce the stochastic drift (banana drift regime). On the rught, the toroidal drift brings in stochasticity (TF stochastic ripple diffusion). 
conserved. Because the CFPs precess toroidally quite extensively (for the toroidal drift rate, ${ }^{8} \omega_{d} \simeq E q / m r R \Omega$, which could mean a toroidal precession up to approximately $\pi$ over a bounce period for CFPs), a vertical drift would bring an additional displacement in the toroidal direction which can be evaluated ${ }^{8}$ by using

$$
\frac{d \phi_{b}}{d r}=2 \theta_{h} \frac{d q}{d r}-2 q \frac{\partial B / \partial r}{\partial B / \partial \theta_{b}}
$$

obtained by differentiating the expression for the orbit arc length between bounce points $\phi_{b}=2 q \theta_{b}$. When the ripple drift term becomes larger than a ripple period, the motion becomes stochastic, i.e. when

$$
\frac{d \phi_{b}}{d r} \Delta r \gtrsim \frac{1}{N}
$$

the vertical steps become decorrelated and random, leading to a net diffusion of particles (see Fig. 2.6). Since $\Delta r$ is proportional to $\delta$, the ripple strength, a criterion can be established for stochastic diffusion. By evaluating equation 2.7 for a low $\beta$, cylindrical case and with the average bounce point located at $\theta \sim \pi / 2$, Goldston, White and Boozer obtained:

$$
\delta>\delta_{s} \equiv\left(\frac{\epsilon}{N \pi q}\right)^{\frac{3}{2}} \frac{1}{\left(2 \rho q^{\prime}\right)}
$$

where $q^{\prime} \equiv d q / d r$. This criterion puts a stringent limit on the maximum ripple strength allowed over the plasma cross-section. ${ }^{2}$

The presence of ripples also invalidates the conservation of the toroidal momentum $P_{\phi}$ and lead to the formation of islands in phase space (see Fig. 2.7). The onset of stochasticity can thus be observed when the islands overlap. The condition for overlap can be calculated theoretically ${ }^{20,21}$ and yields the same criterion (Eq. 2.8) up to a factor of the order of unity (the threshold would be higher by a factor of 1.83). Typically, the criterion corresponds to a region in the outer part of the plasma, called the stochastic ripple domain (or sometimes just as the loss domain). Using the vacuum ripple curves for TFTR ${ }^{22}$ (see Fig. 2.8 which gives the peak to peak value of the ripple $\equiv 2 \delta$ ), we calculated for a $I_{p}=1.4 \mathrm{MA}, B_{T}=4 \mathrm{~T}$ and $R_{o}=2.60 \mathrm{~m}$ discharge, the loss domain which is outlined by a shaded area in Fig. 2.9. Any trapped particle with its bounce point falling in that region would be lost although there is a small 

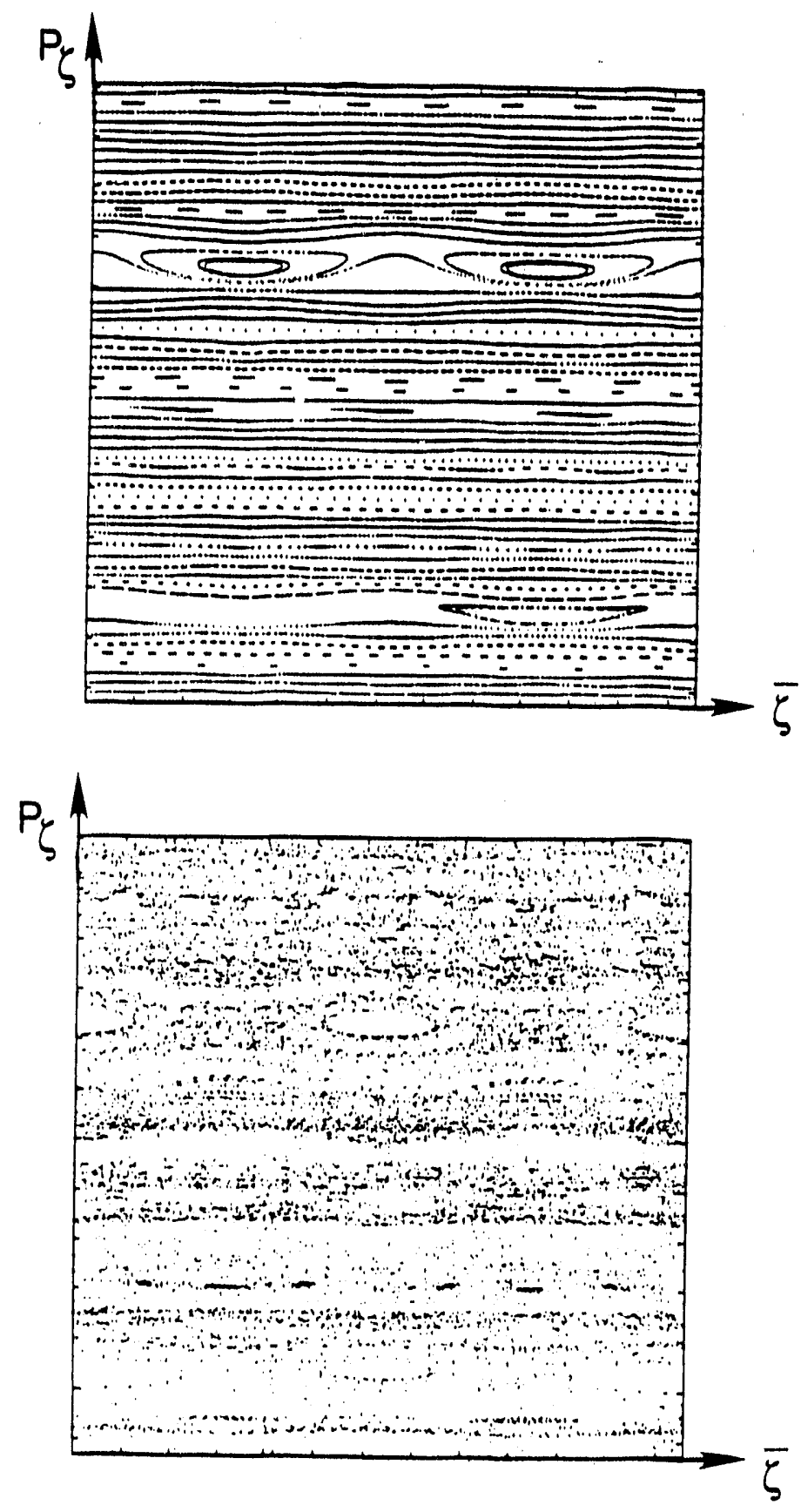

Figure 2.7: Poincaré plot of a Charged Fusion Product orbit in phase space (Duvall ${ }^{181}$ ) illustrating the transition from regular KAM surfaces (islands) to stochasticity (islands overlapping). 


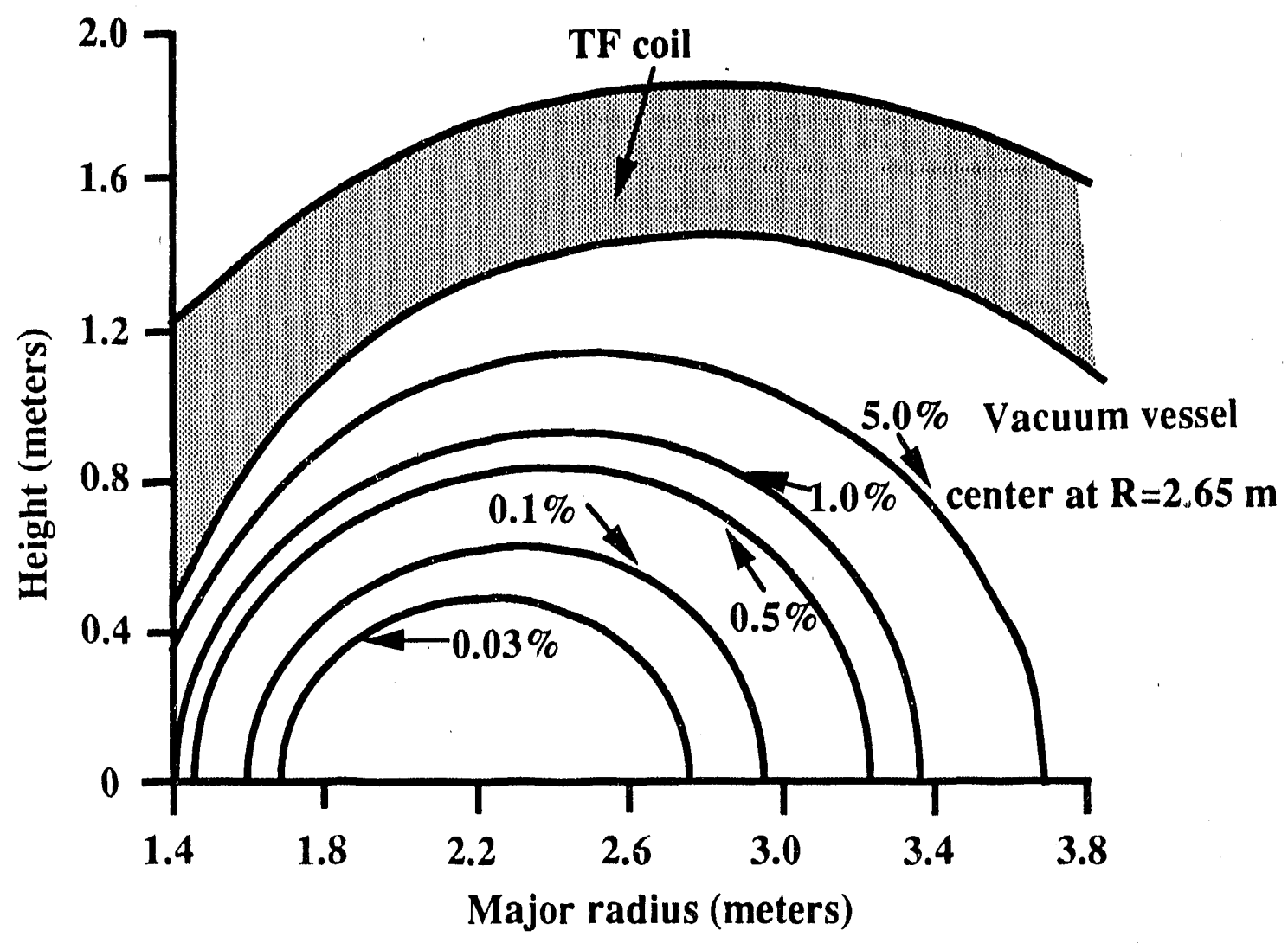

Figure 2.8: lacuum toroidal field ripple curves (peak to peak value in \%) for TFTR.

probability that the particle would stay confined long enough for it to thermalize or pitch angle scatter out of the stochastic domain.

If the loss domain is sufficiently large, this mechanism could theoretically lead to the loss of all the trapped particles, and because $\Delta r$ is relatively large for CFPs (on the order of a centimeter near the plasma edge of TFTR), they will be lost quickly. By using a simple random walk approximation the vertical step size can be used for evaluating an equivalent diffusion coefficient; the diffusion coefficient would be $\mathrm{D}_{\text {ripple }} \sim(\Delta r)^{2} / \tau_{b}$. For TFTR, using $\Delta r \sim 1 \mathrm{~cm}$ and $\tau_{b} \sim 10 \mu \mathrm{sec}, \mathrm{D}_{\text {ripple }} \sim 10 \mathrm{~m}^{2} / \mathrm{sec}$ implying that most of the escaping particles would be lost in typically less than $10 \mathrm{~ms}$ for the $1 \mathrm{MeV}$ triton (and three times less for the $3 \mathrm{MeV}$ proton), which is at least an order of magnitude smaller than their slowing-down time. Consequently, diffusing particles are believed to have nearly their full energy at the wall impact.

For a particle whose barely confined, i.e. that just misses the outer midplane on its orbit, and which receives a vertical kick of a centimeter or so at its bounce point (if directed outwards) would bring it to hit predominantly just below the outer 


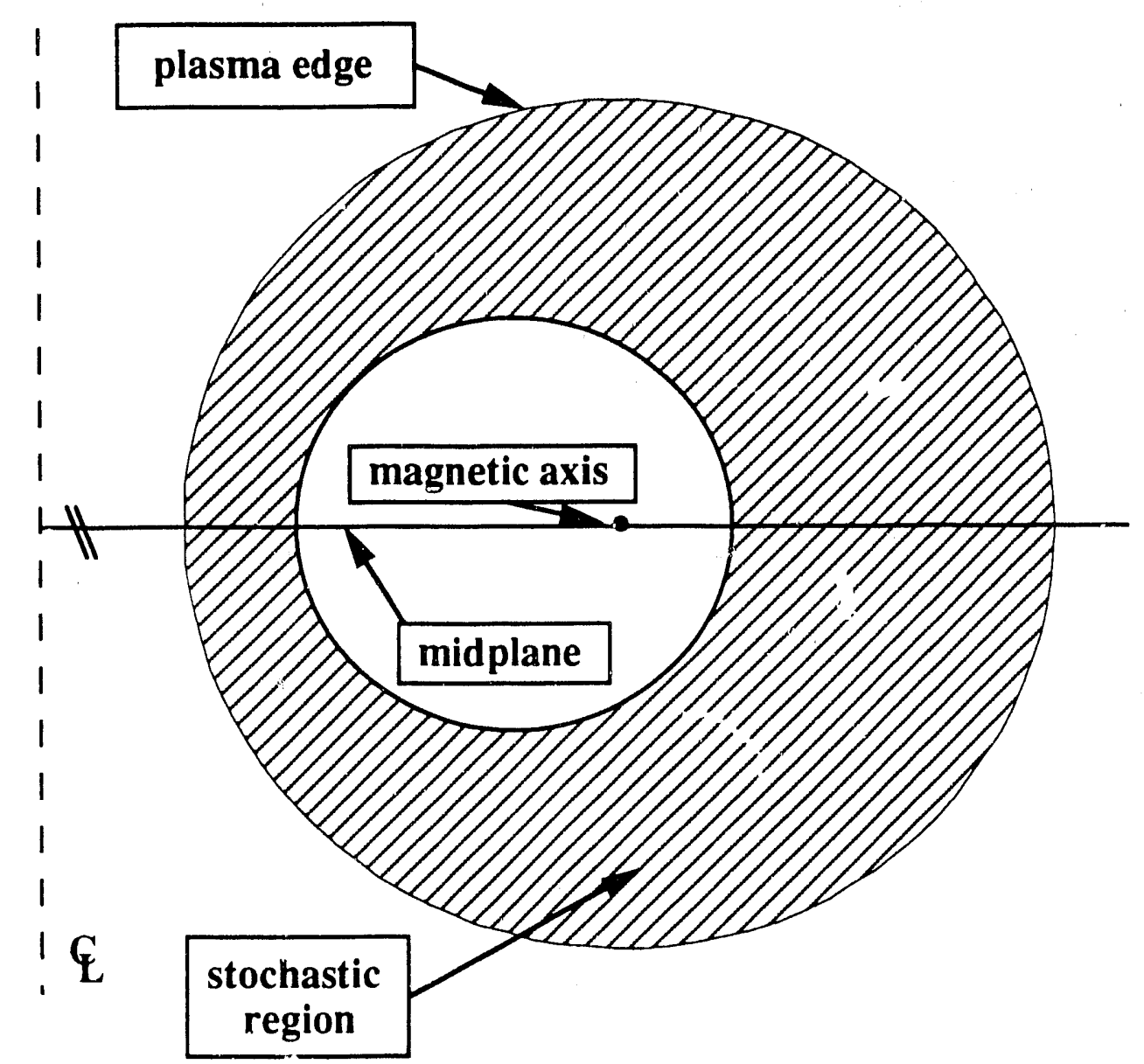

Figure 2.9: Poloidal cross-section of the loss domain for stochastic diffusion (lined area) for a typical $I_{p}=1.4 M A, B_{T}=4 T$ and $R_{0}=2.60 \mathrm{~m}$ TFTR discharge. 


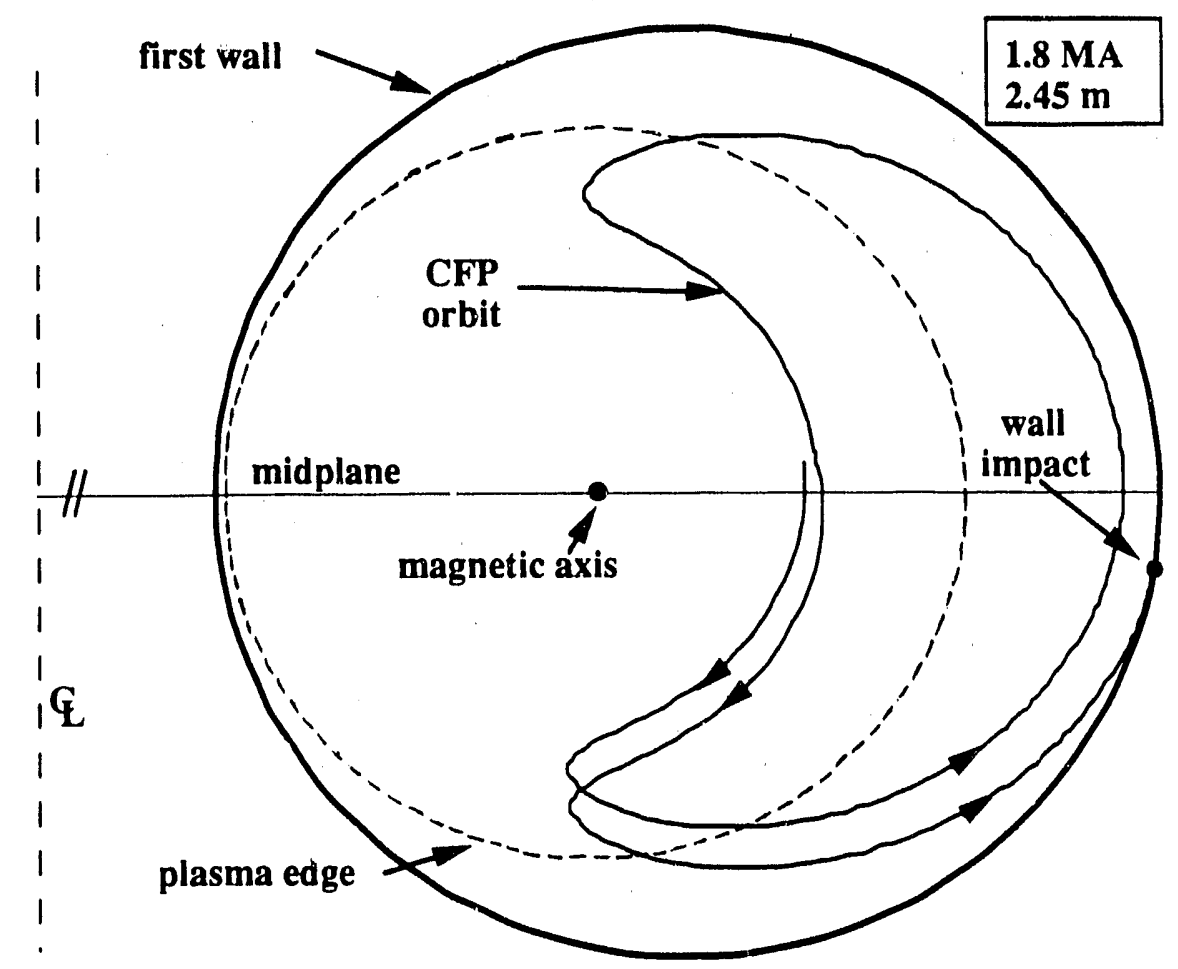

Figure 2.10: Poloidal cross-section of TFTR showing the likely location of impact for a ripple lost particle. The CFP misses the wall on one pass and hit it on the next one just below the outer midplane.

midplane (or just above, depending on the ion $\nabla \mathrm{B}$ drift direction). This localization of stochastic ripple losses represents a serious problem of heat loads on the first wall of fusion reactors. Even globally small losses of alpha particles (< few percent) can lead to serious heat loads near the outer midplane, in ITER for example. ${ }^{6,121}$ This effect is illustrated on Fig. 2.10, where a trapped CFP just missed the outer midplane on the previous pass but impact the wall on the next one. The distribution of impacts along the first wall is mainly a function of the last vertical step size $\Delta r$, the banar 2 width $\Delta_{b}$ and the spatial origin of the escaping ions.

Numerical calculations for ripple diffusion were undertaken by several groups across the world using different approaches. Many of them were based on a particle guiding center integration scheme (for example Tani et al, ${ }^{4}$ Bittoni and Haegi, ${ }^{23}, 24$ Hively ${ }^{25,26}$ and Rome ${ }^{27}$ ). Others used mapping techniques (Goloborod'ko et $\mathrm{al}^{3}$ ) or used the stochastic threshold criterion only, for global calculations (as in Mynick and White's code RIPLOS. ${ }^{28}$ ) 


\subsubsection{Turbulent diffusion}

It is known that when the magnetic field structure is axisymmetric and unperturbed, particle orbits close back on themselves poloidally after many transit periods and would be affected only by collisions. As we saw in the previous section, irregularities in the field structure can arise from the discrete number of field coils. In that case, the perturbations are a feature external to the plasm. However, the plasma itself can perturb the orbits through magnetic or electrostatic turbulence. The perturbations can provoke radial diffusion and potentially prevent the full thermalization of alpha particles. This mechanism would affect all particles but could be dominated by other processes; for instance, for trapped particles TF ripple diffusion would be a faster process. However, for passing particles, turbulence-induced diffusion may be the most "serious" candidate.

This diffusion can increase the amount of losses by eventually bringing particles to the wall, essentially at the inner or outer midplane, or by bringing them to a existing loss cone, i.e. first-orbit losses, by crossing the passing-trapped boundary. However recent calculations ${ }^{29,19}$ indicate that such a process should be small for CFPs; diffusion would not prevent thermalization of alpha particles in reactor grade tokamaks where particles would slow down before reaching any existing loss cone. It is conjectured that since the gyroradius of these particles (typically $5-10 \mathrm{~cm}$ ) is much larger that the turbulence scalelength $(\sim 1 \mathrm{~cm}$ or less) the turbulence would be averaged out by the particle's gyromotion. Consequently, the turbulent diffusion coefficient would be greatly reduced compared to the thermal ions. Numerical calculations ${ }^{19}$ indicate that in the case of TFTR, $\mathrm{D}<10^{-4} \mathrm{~m}^{2} / \mathrm{sec}$ which is small compared to the experimentally obtained coefficient for thermal ions, $D_{\text {thermal }} \sim 1 \mathrm{~m}^{2} / \mathrm{sec}$.

\subsubsection{Other diffusion mechanisms}

Other mechanisms for CFP losses have been studied, observed or simply suggested. As for the TF ripple and turbulence-induced diffusion many of those processes rely on an existing loss cone into which previously confined particles are thrown. Additional losses could be due to the presence of large coherent magnetohydrodynamic (MHD) activity (i.e. kink modes, fishbones, sawteeth, ballooning modes, toroidal Alfvén eigenmode (TAE), etc.) or the action of collisions. 
The effects of these different mechanisms can be best illustrated by looking at Fig. 2.11 which is based on Fig. 2.3. The effects of a large coherent mode (or a combination of modes) can be examined by looking at particles located near a resonance layer. It is been conjectured ${ }^{19}$ that with the introduction of a radial magnetic field from the magnetic perturbations, passing particles receive a radial "kick". If $\mu / \mu_{0}$ is conserved, particles would then only move horizontally on Fig. 2.11 so they can cross the trapped/passing boundary and are thus very quickly lost to the wall. It is easy to realize that the larger the resonance layer is, or the stronger the mode is, the more particles would be lost by this process as more of the passing/trapped boundary would be covered. This can represent a problem for the confinement of alpha-like particles ${ }^{30}$ but the amplitude of the losses has not been quantified yet.

Pitch-angle scattering, would "move" particles, mainly vertically on Fig. 2.11, by changing their $\mu / \mu_{0}$. Particles near the passing/trapped boundary can be "kicked" into the loss cone from an initially passing confined particles. Because such a mechanism is localized in that plane and because the number of collisions is so small for CFPs, it is believed that losses should be negligible for particles near their birth energy. However, both terms (slowing-down and pitch-angle scattering) must be kept when one calculates the diffusion (and consequently the losses) over the slowing down

time. $^{31,32,33}$ Nevertheless, this neoclassical diffusion would still be relatively small for CFPs, i.e. in TFTR. ${ }^{34}$

Sawteeth are also believed to alter the confinement of CFPs through a relatively complex mechanism. As the sawtooth process is by itself not completely understood it is difficult to predict its effects on the CFPs. ${ }^{35}$ In fact some information on the sawtooth mechanism could be inferred from the study of escaping alpha-like particles during a sawtooth crash. At this point too little is known to even sketch the process on Fig. 2.11.

\subsection{Previous experimental results}

The experimental study of the confinement of CFPs in tokamaks is not an easy task. Many points contribute to complicate the measurements. The most important one is naturally the number of fusion reactions; the plasma temperature (in a D-D or D-T mix) must be high enough to produce fusion events in large number. Another problem resides in the access to those particles; CFPs are contained within the vacuum 


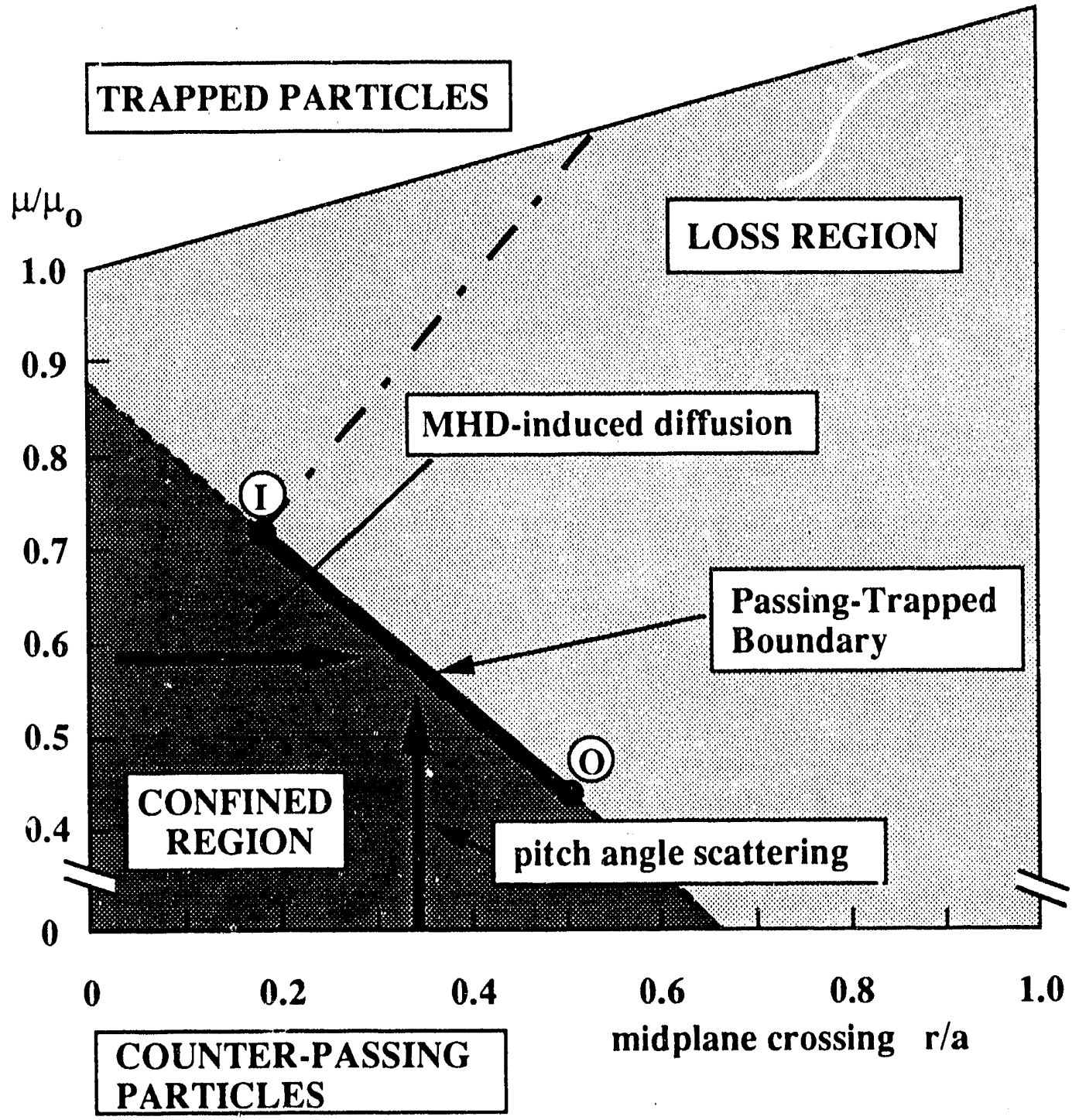

Figure 2.11: Illustration of the MHD and pitch angle loss processes. MHD activity will move particles horizontally (keeping $\mu$ constant), towards to the loss cone (passing-trapped boundary). Pitch angle scattering would have a similar effect but more on a vertical trajectory. 
vessel and detectors must be installed within the first wall of the tokamak. This requirement also puts limits on the type of detectors that can be used because of temperature, vacuum or neutron handling capabilities. The only exception to the in-vessel detection is in the use of secondary nuclear reactions (neutron detection from triton burn-up, ${ }^{36,37,38}$ gamma ray detection from nuclear reactions with doping elements $\left.(\mathrm{Li}, \mathrm{B})^{39}\right)$. Other diagnostics are under development to study the confined alpha populations ${ }^{40,} 41,42$ but will be only useful at large alpha particles densities (for example with the use of tritium as one of the fuels).

Pioneering work on the confinement of CFPs was done by Strachan ${ }^{43}$ on PLT and PDX (and later on PBX) with Chrien ${ }^{44-48}$ Heidbrink $^{14,49-55}$ and later with Lovberg ${ }^{56}$ and Murphy. ${ }^{57}$ Their work will be described briefly in section 2.2.1. Work on TFTR has been done by Strachan and Barnes on burn-up and by Zweben on losses of CFPs. On JET some experiments were performed, many on burn-up by Sadler, ${ }^{36}$ Conroy ${ }^{58}$ and Kaëllne ${ }^{37}$ and on RF heated D- ${ }^{3} \mathrm{He}$ discharges by Martin. ${ }^{59}$ Bosch performed also experiments on ASDEX ${ }^{60,61}$ on protons and tritons. Preliminary results have been reported from $\mathrm{T}-10^{62}$ and a new detector has been recently installed on Tore-Supra. ${ }^{63}$

\subsubsection{First-orbit losses}

In small to medium scale machines (like PLT, PDX, PBX, T-10, and ASDEX) loss mechanisms are dominated by first-orbit losses, mainly because of their relatively low plasma current. Many experiments were performed to study the losses of CFPs in those machines. As anomalous losses are always negligible compared to first-orbit losses in those tokamaks it was at least theoretically possible to study the plasma itself (as enumerated below) by looking at the escaping flux of CFPs.

In the large majority of these experiments, surface barrier detectors (SBDs) were used inside the vacuum vessel to look at the escaping flux of CFPs. By analyzing the energy distribution and the pitch angle distribution of the detected particles they were able to study experimentally, the ion temperature, the ion distribution function ${ }^{64}$ the ion temperature gradient, the poloidal field structure,$^{14}$ the helium density and the source profile of alphas from the RF heated $\mathrm{D}-{ }^{3} \mathrm{He}$ discharges.

In larger machines (i.e. TFTR and JET, and potentially on Tore-Supra and JT$60 \mathrm{U})$ tritons, protons $(3 \mathrm{MeV})$ and alphas are largely confined (at least on their first-orbit) so the emphasis is shifted from the siudy of the plasma through the CFPs 
to the study of the CFP loss mechanisms themselves. One CFP still largely unconfined in those machines is the $14.7 \mathrm{MeV}$ proton from the D- ${ }^{3} \mathrm{He}$ reaction. Experiments in TFTR $^{65}$ and JET $^{66}$ (both by using SBDs), have been performed on that particle and were used to study helium transport ${ }^{67}$ and the effects of sawteeth. ${ }^{59}$ These detectors were also used to study the burn-up of the $0.8 \mathrm{MeV}{ }^{3} \mathrm{He}^{68}$ The burn-up studies cover also the $14 \mathrm{MeV}$ neutron (burn-up of the $1 \mathrm{MeV}$ triton) which started on PLT $^{38,49}$ and PDX ${ }^{49,51}$ and continued on TFTR ${ }^{69}$ and JET. ${ }^{36,37,58,59}$ However, these measurements lack time resolution (if the activation technique is used) or cannot be spatially resolved. For example, anomalous triton burn-up was found in TFTR, ${ }^{69}$ but has not yet been directly attributed to a specific loss mechanism, mainly since it is only a global measurement.

Finally, collector data have been performed on $\mathrm{TFTR}^{70}$ and on $\mathrm{JET}^{71,72}$ to study the confinement of energetic ions. These measurements were made by exposing samples, usually silicon, nickel or carbun, during many shots. Particle fluence is then measured using the SIMS ${ }^{73,74}$ technique. These measurements tend to be of limited scope (no time resolution and long exposures) and are mainly used for global trend checks with more direct measurements or to look at the effects of long exposures to flux of energetic ions on first wall materials.

\subsubsection{Toroidal field ripple losses}

Since ripple diffusion is expected to affect only some classes of particles and since those effects should be visible only at specific locations, special diagnostics have to be designed for its study. As mentioned earlier, so far only results on collisional processes (ripple plateau and banana drift regimes) have been reported. This research has also been limited to fast ions (all with $E_{\text {ions }} \lesssim 35 \mathrm{keV}$ ), no results have been reported on the effect of ripples on the confinement of CFPs.

The first attempts to look experimentally at the ripple effects in a non-axisymmetric system were performed on $\mathrm{T} 3-\mathrm{A},{ }^{75} \mathrm{ST}^{76}$ and later on $\mathrm{PLT}^{77,}{ }^{78}$ but consisted in modifying the field only locally, i.e. by changing the current in one toroidal coil. The effects were studied mainly with global parameters (temperature, resistivity) and little on the confinement of ions through charge-exchange analyzers. No major effects were found except in the presence of large ripples $(>20 \%)$. The absence of effect was later explained by the low n number of the perturbation, which kept the stochastic 
threshold very high and could not lead to the formation of ripple trapped particles.

Experiments were pursued on $\mathrm{T}-4^{79,80}$ and $\mathrm{T}-10^{81,82}$ using charge-exchange analyzers, and modifications were observed in the ion energy distribution $\left(E_{\text {ions }} \leqslant 3\right.$ $\mathrm{keV}$ ) and compared with Fokker-Planck calculations of ripple transport. At about the same time, experiments were performed by Scott on the ISX-B tokamak ${ }^{83} 80$ with a similar approach. Plasma parameters were studied in low and high ripple conditions, varied by removing half the TF coils from the circuit. Fast ion losses were monitored by charge-exchange analyzers for ion energies below $30 \mathrm{keV}$. Results were found to be consistent with collisional ripple transport calculations also made with a Fokker-Planck code. Charge-exchange measurements of the ion energy distribution were also done on Alcator $A^{85}$ and it was conjectured that the observed deviation from a Maxwellian distribution probably originated from ripple transport.

A different approach was taken at TFR where special detectors were used to study the flux of fast ions to the wall. In one experiment a set of five biased collectors were poloidally distributed at the bottom of $\mathrm{TFR}^{86}$ at one single toroidal location. A large and anomalous ion flux was found during ICRF heating and, because of the detector location and geometry, is believed to be due to ripple trapping and/or collisional ripple transport. A more complex detection system was used in a subsequent experiment ${ }^{87,88}$ and was composed of a set of six Faraday cups installed between two coils at the top of TFR. The design of these detectors was such that only a small class of particles could reach them, including ripple diffusing ions. Experimental results agreed, within the uncertainties, with a ripple transport model (including ripple trapping and banana drift processes) based on Fokker-Planck and Monte-Carlo calculations.

Recently observed hot spots below the bottom first wall of Tore-Supra were attributed to drifting ripple trapped particles. Calculations using guiding center equations were consistent with the ripple trapping of $\gtrsim 50 \mathrm{keV}$ electrons and a rapid loss to a localized region on the wall. ${ }^{89}$

\subsubsection{Turbulence-induced diffusion}

The original poloidal array of CFP detectors installed by Zweben ${ }^{90}$ was first used to study first-orbit losses. ${ }^{91}$ Subsequently, one of the detector (bottom \#6) was used more specifically to look at the possible turbulent diffusion of confined passing particles. The diffusion of these particles would manifest itself by an additional escaping 
flux of CFPs visible at the pitch angle corresponding to the fattest banana, at the boundary between passing and trapped particles. Passing particles, normally confined near the center of the pli ma, would diffuse radially out until they mirror (becoming trapped), and then could reach the first wall of the tokamak through the much wider trapped particle orbit. The bottom detector was used to look for signatures of this process, through anomalous losses at high current (where first-orbit losses are minimized) and through the pitch angle distribution which would be distorted by an additional flux of escaping particles at the fattest banana pitch angle. The observed absence of such signatures ${ }^{34,92}$ was used to put an upper bound on the diffusion rate of passing particles, at the relatively low level of $D_{C F P}<0.1 \mathrm{~m}^{2} / \mathrm{sec}$ which is much lower than the experimentally found level for thermal particles $\left(D_{\text {thermal }} \sim 1 \mathrm{~m}^{2} / \mathrm{sec}\right)$.

\subsubsection{Other loss mechanisms}

Large coherent MHD activity has been observed to induce anomalous CFP losses in TFTR. ${ }^{91,93,94}$ These observalions have been made with the bottom detector $(\theta=$ $-90^{\circ}$ ) and were found to be modulated by the magnetic oscillations as observed by Mirnov coils. The MHD-induced losses were also found to be induced by a variety of different modes (kink, fishbones) and seem to occur at the pitch angle corresponding to the passing/trapped boundary. No systematic study of this effect has been done yet, but preliminary theoretical work indicates a mechanism by which passing particle would become trapped and then rapidly lost, after having received a radial kick from the magnetic perturbation. ${ }^{19}$

Another anomalous loss process has been observed by two detectors at the bottom $\left(\theta=-90^{\circ}\right.$ and $\left.-60^{\circ}\right)$. Additional losses were induced away from the normal peak in first-orbit losses (fattest banana), at an higher pitch angle and at an apparently lower energy. This phenomenon appeared unpredictably and seemed to be connected with the presence of MHD activity. Some possible parallel with a ripple loss mechanism will be briefly discussed in Ch. 7 along with some experimental results.

Sawteeth were also observed to induce anomalous losses of CFPs (under the form of bursts), both on TFTR ${ }^{93,94}$ and on JET. ${ }^{59}$ In the former case they observed escaping $1 \mathrm{MeV}$ tritons and $3 \mathrm{MeV}$ protons, whereas in latter case, $14.7 \mathrm{MeV}$ protons were studied. In JET, this effect could be due to the ejection of the CFPs themselves or of the energetic fuel ions which would fuse much farther out from the plasma center 
where the CFPs would normally be confined. 


\section{Chapter 3}

\section{Experimental technique}

As we saw in the previous chapter the CFPs are contained within the vacuum vessel and the study of their confinement (or lack of) is complicated by their relatively complex orbits. Diffusion and loss mechanisms can only be understood efficiently if we can retrace their orbit backward in time from a detector. We saw also in the previous chapter that by solving the equations of motion (or by using the constants of motion $\mu, E$ and $P_{\phi}$ if the tokamak is axisymmetric) one can establish the trajectory of the CFPs. Basically one particle has six degrees of freedom and so we need to have six initial conditions (or final if we want to retrace the orbits backwards in time), three in coordinate space and three in phase space. Since charged particles gyrate around a local field line we can limit the necessary information in phase space to the parallel and perpendicular velocities. 'That means that for any particle location (usually the impact location in the detector), we need to know its energy and magnetic moment, and also its precise position. We wish also to have time resolution of the escaping flux so that time-dependence of diffusion and losses can be studied. All those requirements plus the constraints of the tokamak environment put limits on the type of detectors that can be used. For example, the use of surface barrier diodes (SBD, also called silicon barrier detectors) was not feasible due to the harsh neutron environment at the first wall of TFTR.

The technique that has been adopted uses a scintillator, in our case the $\operatorname{ZnS}(\mathrm{Ag})$ (also known as P-11), which has many of the required features. From the point of view of the environment, this scintillator is good in a high vacuum, high neutron environment, has no interference with electromagnetic fields (optical coupling) and does not suffer permanent damage from being exposed to high temperature $\left(<500^{\circ} \mathrm{C}\right){ }^{95}$ 
For our measurement the $\mathrm{ZnS}(\mathrm{Ag})$ provides a very good scintillation efficiency (between $10 \%$ and $20 \%^{96,97}$ of the particle energy is transformed into light), and a good time resolution $(<10 \mu \mathrm{sec})$, but unfortunately, because of its multi-crystal structure, it has a poor intrinsic energy resolution. We will present in the Section 3.1 the details of the detector design including the technique for energy resolution.

\subsection{Detection system description}

As we mentioned in the previous section the $\mathrm{ZnS}(\mathrm{Ag})$ scintillator has an insufficient energy resolution and so the design of the apertures must compensate for this deficiency. The technique uses a pinhole and a slit through which particles can be discriminated according to their gyroradii, ard to their pitch angle in the orthogonal direction. This design was first used on PLT for measuring escaping alpha particles produced in the $\mathrm{D}^{3} \mathrm{He}$ reaction. ${ }^{57}$ In their design, a plastic nuclear track detector (CR-39) was used instead of a scintillator. The major drawbacks of the nucleal track detector were the absence of time resolution and the necessary development process.

In Fig. 3.1 is shown the basic design of our detector. The $10 \mu \mathrm{m}$ thick $\mathrm{ZnS}(\mathrm{Ag})$ is located at the top of the box with its plane parallel and slightly above the set of slit and pinhole. Particles gyrating around the field line would penetrate through the pinhole and the slit and then would impact on the scintillator. The light ernitted by the scintillator is transmitted by a system of lenses and fiber optics to an intensified camera and/or photomultiplier tubes.

In 1987, Zweben installed four detectors in a poloidal array, ${ }^{90}$ based on that design, at the bottom of TFTR bay $\mathbf{E}$ shown in Fig. 3.2. Of the original four, three were kept operational (the most inner one has been temporarily "disconnected" since it was designed to look only at the $14.7 \mathrm{MeV}$ proton) and are all in a fixed position slightly behind the radius of the RF limiters. They will be described briefly in Section 3.1.2. The midplane probe is similar in design to the bottom detectors and located in the same bay (see Fig. 3.2). It is inserted within the first wall through the penetration shown just below the midplane. Virtually all the experimental results presented in this thesis were obtained with this midplane probe. A more detailed description of the detector will be presented in the next two sections. 


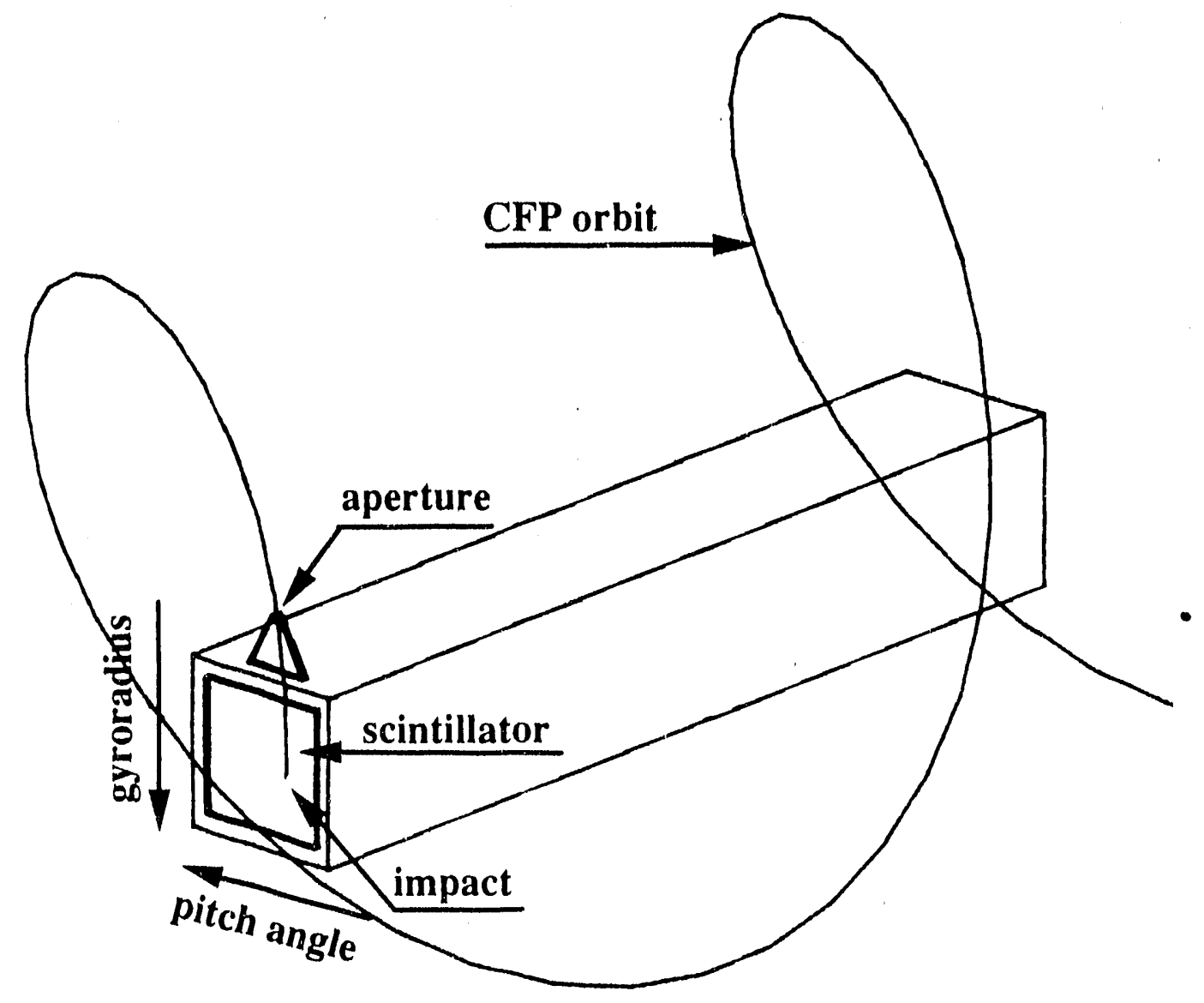

Figure 3.1: Schematic diagram of the detector design. Components are roughly to scale, including a typical CFP orbit (with a gyroradius $\approx 7.5 \mathrm{~cm}$ and $60^{\circ}$ pitch angle). 


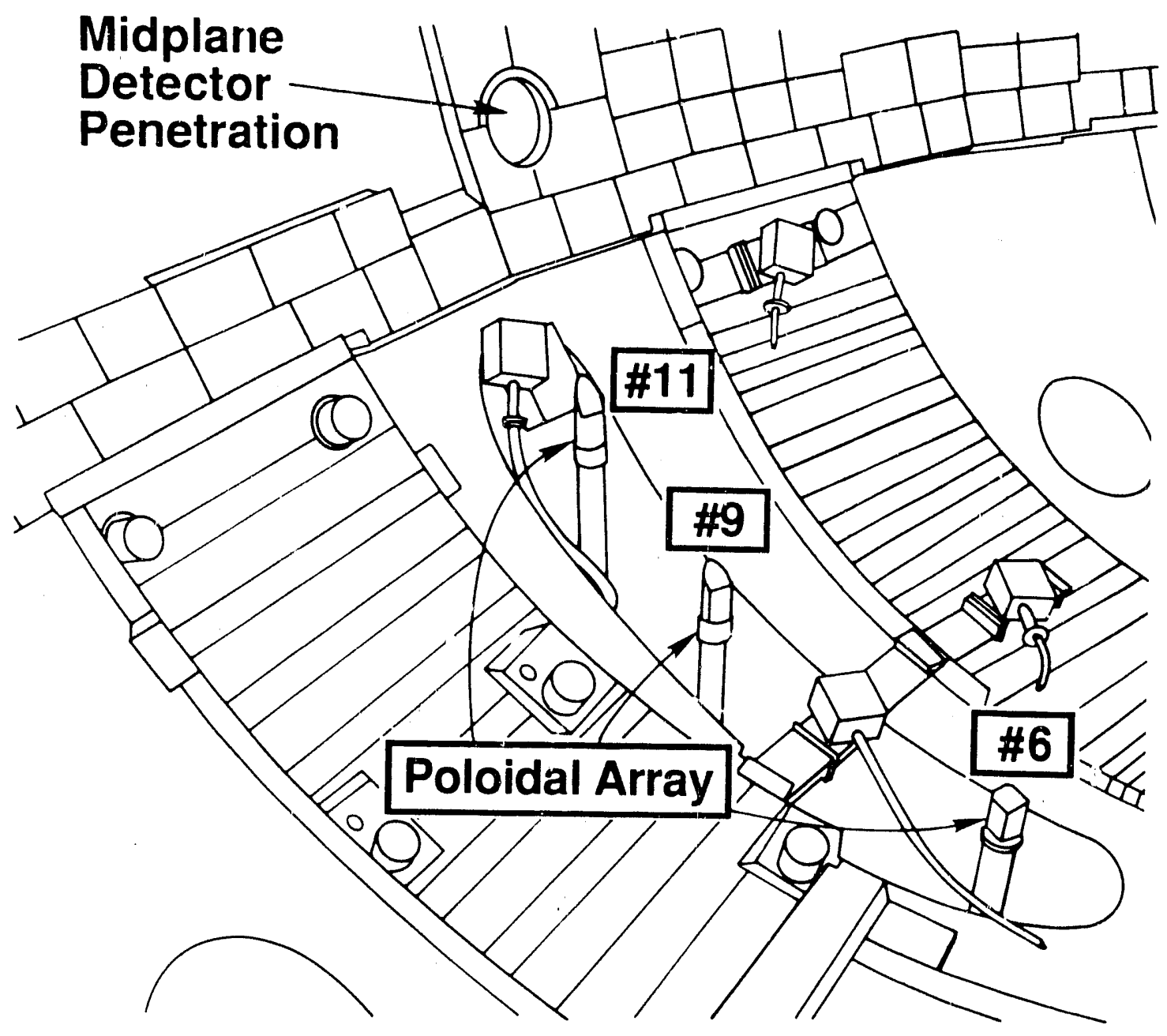

Figure 3.2: Schematic diagram of the location of the detectors at the bottom of TFTR bay $\mathrm{E}$. Indicated are the three detectors (\#6, \#9 and \#11) forming the poloidal array and the penetration (top) for the midplane probe. 


\subsubsection{Midplane probe}

Of the poloidal array of fixed detectors installed at the bottom of TFTR, the closest to the outer midplane is located at $\theta=-45^{\circ}$. This relatively large distance from the outer midplane would leave a large region not covered by any detector. The study of certain loss mechanisms would be impossible. For example, the TF stochastic ripple losses are expected to occur predominantly between $\theta \simeq-10^{\circ}$ and $\theta \simeq-30^{\circ}$, just below the outer midplane. ${ }^{3}$

In 1989, a probe previously used by Manos and Kilpatrick for edge measure ments $^{98,99}$ (Langmuir probe, sample exposures, and calorimetry), was modified for measuring CFP populations near the first wall. The probe is located $35.6 \mathrm{~cm}$ below the midplane, which corresponds to a poloidal angle of $-20^{\circ}$ at the first wall. Shown in Fig. 3.3 is a picture of the final assembly before the first installation. On the right, is shown the probe head and its armor. A close-up view of the probe head is shown in Fig. 3.4. The probe is mounted on a torus interface valve (TIV) which allows easy removal without a vacuum vessel opening. The present stroke of the probe is 1.13 .7 $\mathrm{cm}$ (which can be slightly extended if the need arises) of which $23 \mathrm{~cm}$ are with the aperture within the vacuum vessel first wall. Of those $23 \mathrm{~cm}$, the first 9 lie in the shadow of the RF limiters, which are located in two different bays approximately $117^{\circ}$ and $171^{\circ}$ away toroidally (in the counter-going direction) and approximately $50 \mathrm{~cm}$ wide. All the positions were measured inside the vacuum vessel during the winter 1991 opening using a specially designed articulated measuring arm (with an accuracy of better than $.3 \mathrm{~cm}$ ) and supersede previous position calculations. ${ }^{100}$ The probe position was also monitored during some of the discharges and no radial motion was found. However, small lateral or vertical motions ( $\leqslant$ few $\mathrm{mm}$ ) could still be present during a shot.

The important feature of this detector compared with the bottom detectors, is precisely its ability to move. The probe can be brought very close to the plasma, although it was always kept at a safe distance $(Z 5 \mathrm{~cm})$ because it was not designed to withstand excessive heat. The position of the probe was kept constant during individual shots, mainly because the driving mechanism is too slow (approximately $0.5 \mathrm{~cm} / \mathrm{sec}$ ) and there is no direct fast timescale pusition recording.

This moving ability enables the detector to look at two different classes of particles that we can simply describe as "lost" and "confined". In the former case, the probe 


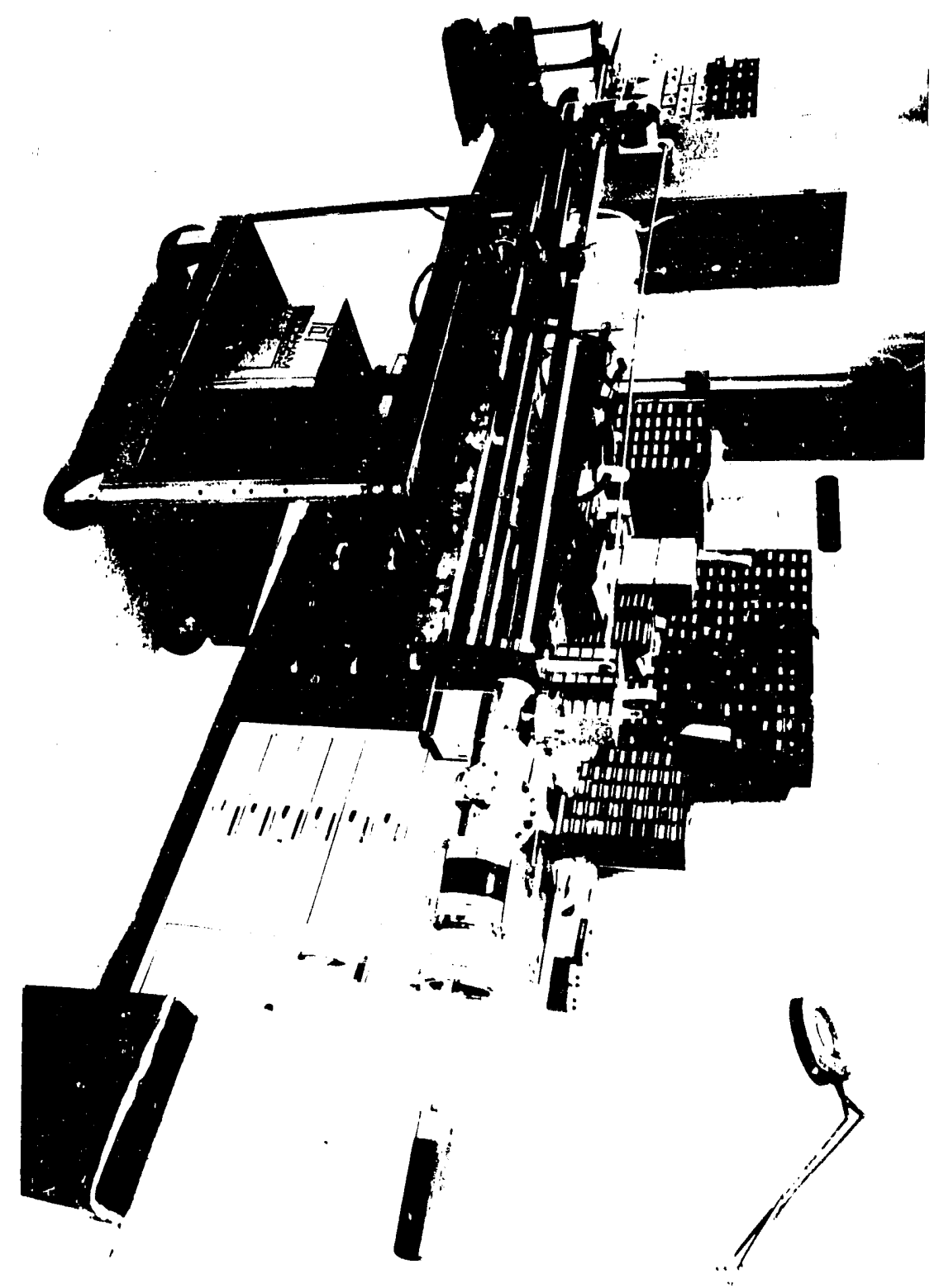

Figure 3.3: Final assembly of the midplane probe before installation. 


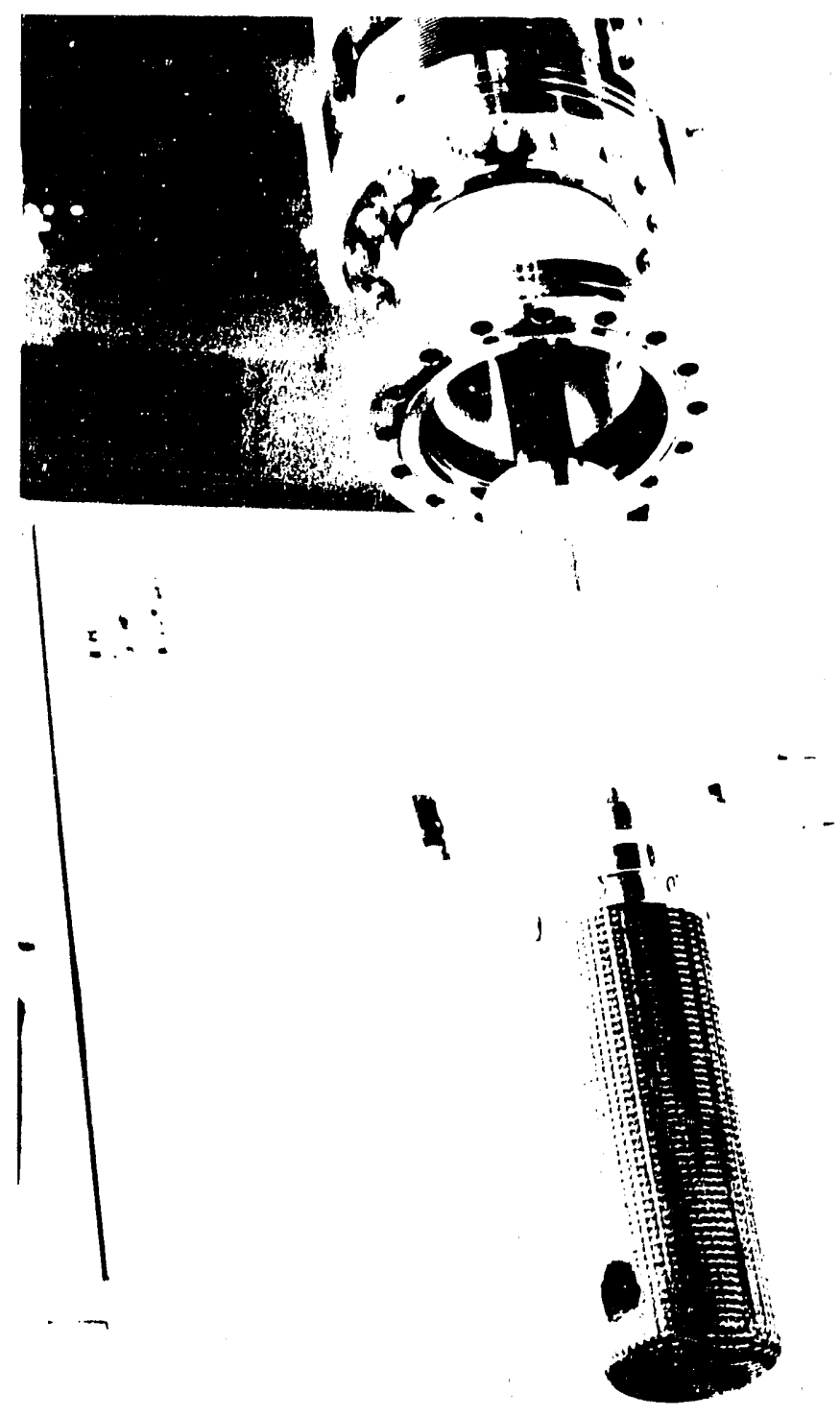

Figure 3.4: Enlarged vieu of the probe head after assembly shoung the aperture near. the probe tip. 


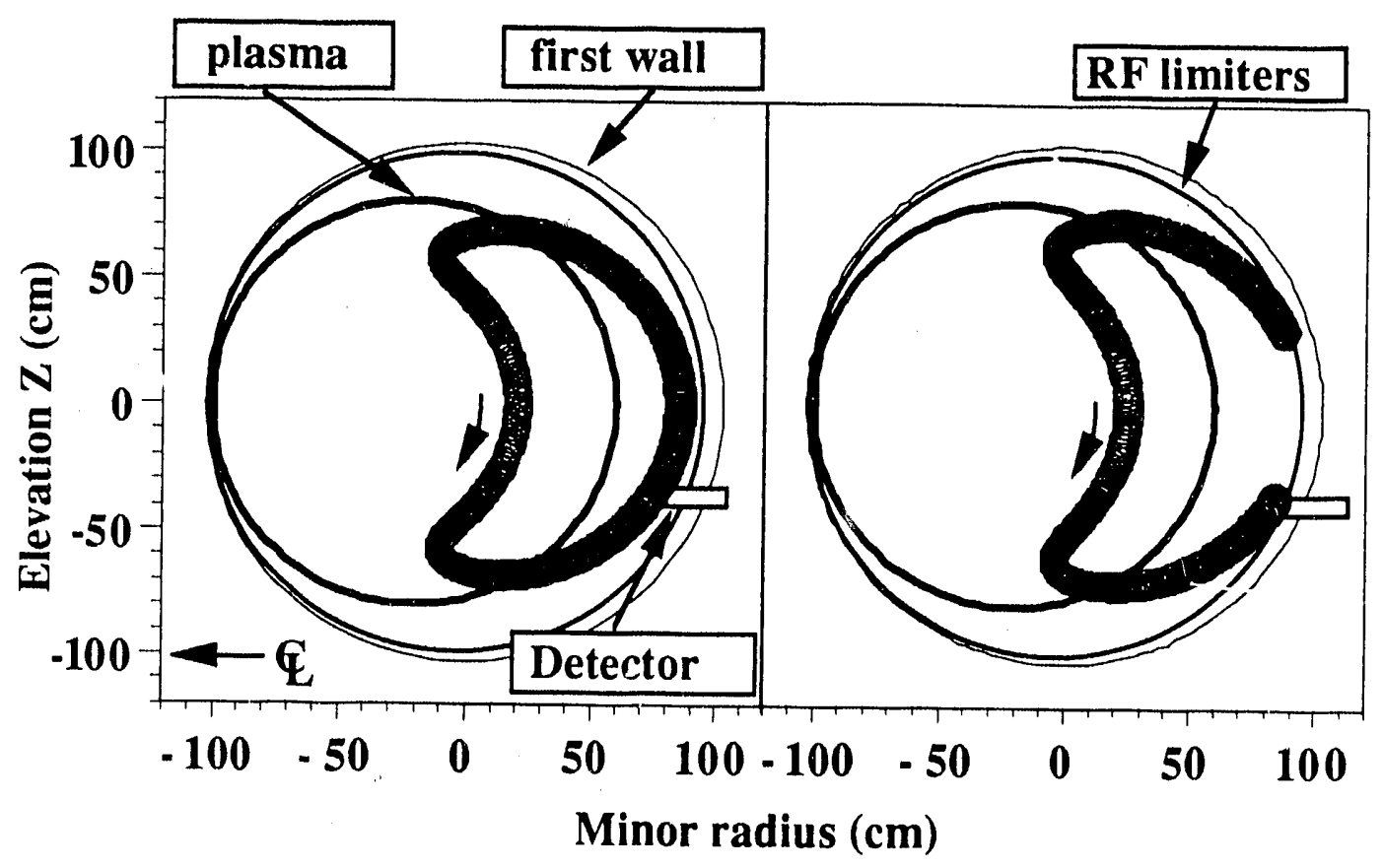

Figure 3.5: Typical orbits seen by the detector at two different radial positions showing the difference between a confined first orbit (left) and an orbit on a direct path to the wall (right).

is usually located behind the RF limiters radius and detects particles on their direct way to the wall (see Fig. 3.5). In the latter case, when the probe is located inside the RF limiters radius, there is no material obstacle between the aperture and the plasma (except for the detector itself), the detector then samples confined orbits (because of their large banana widths CFPs born in the plasma can be found relatively far out from the plasma), or possibly CFPs diffusing out (see Fig. 3.5). This detector is the first diagnostic capable of looking at some part of the confined CFP population.

Shown in Fig. 3.6 is the mechanical layout of the probe and its internal parts. In the probe head, at the extreme left on the diagram are the scintillator, the set of collimating apertures and the mechanical support. Further down the probe we have the quartz lens which images the scintillator onto a $2 \mathrm{~m}$ long coherent fiber optic bundle made of $20 \times 20$ quartz optic fibers (polyamide jacketed, $0.5 \mathrm{~mm}$ in diameter*).

\footnotetext{
'The optic fibers were fabricated by Polymicro Technologies, Phoenix, AZ 85017 and the bundle
} 
This bundle carries the light to the sapphire window where the vacuum ends. Another quartz lens, located on the other side of the window, transmits the image to another coherent fiber optic bundle, this time $20 \mathrm{~m}$ long, made of $50 \times 50$ quartz optical fibers $0.25 \mathrm{~mm}$ in diameter. The bundle then carries the light to the camera or photomultiplier tubes located in the basement of TFTR.

An enlarged view of the top of the probe head is shown on Fig. 3.7 to illustrate the complete geometry of the apertures, scintillator, support and protection. The set of apertures shown here is the final version adopted in May 1990 which is very slightly different than the one used during the shake-down phase (January-February 1990) and the ones used in the fixed bottom detectors. Details of the aperture system is shown in Fig. 3.8. Also shown is the very thin aluminum foil ( $3 \mu \mathrm{m}$ thick), used to block visible light, which stops the $0.8{ }^{3} \mathrm{He}$, but lets the tritons $(>0.35 \mathrm{MeV})$, the protons $(>0.3 \mathrm{MeV})$ and the alphas $(>0.9 \mathrm{MeV})$ pass. The foil transmission is described in more details in Sections 3.2.1 and B.3. Care was also taken that no direct line of sight existed between the scintillator and outside the detector in order to avoid X-rays backgrounds. The rotation angle of the probe with respect to its own axis, was chosen to be $28.2^{\circ}$ in the counter-going direction, after preliminary orbit calculations ${ }^{101}$ indicated the region of interest in pitch angles.

A code has also been developed by Diesso ${ }^{91}$ to map the different ion orbits inside the detector depending on their pitch angle and gyroradius. Shown in Fig. 3.9 is the centroid location (averaged over both coordinates) of the ion impacts for given pitch angle and gyroradius. This mapping is used to infer the escaping particle "parameters", namely its magnetic moment and energy, from the measured light flux pattern.

The same code can be used to calculate the geometrical resolution for a given set of apertures and detector orientation. In Fig. 3.10 is shown the geometrical spread in pitch angle for the midplane probe (for a pitch angle of $60^{\circ}$ and gyroradius of $7 \mathrm{~cm}$ ) due to the finite width of the pinhole. In this case the spread follows approximately a gaussian distribution with a FWHM of $4.5^{\circ}$. The same can be done for the gyroradius distribution, shown in Fig. 3.10 (for a gyroradius of $7 \mathrm{~cm}$ ) which is mainly due to finite height of both the pinhole and slit and illustrates the poor absolute energy resolution of this detector (no better than $50 \%$ ). Resolution in one or both variables

assembled by Highlight Fiber Optics, Caldwell, ID 83605. 

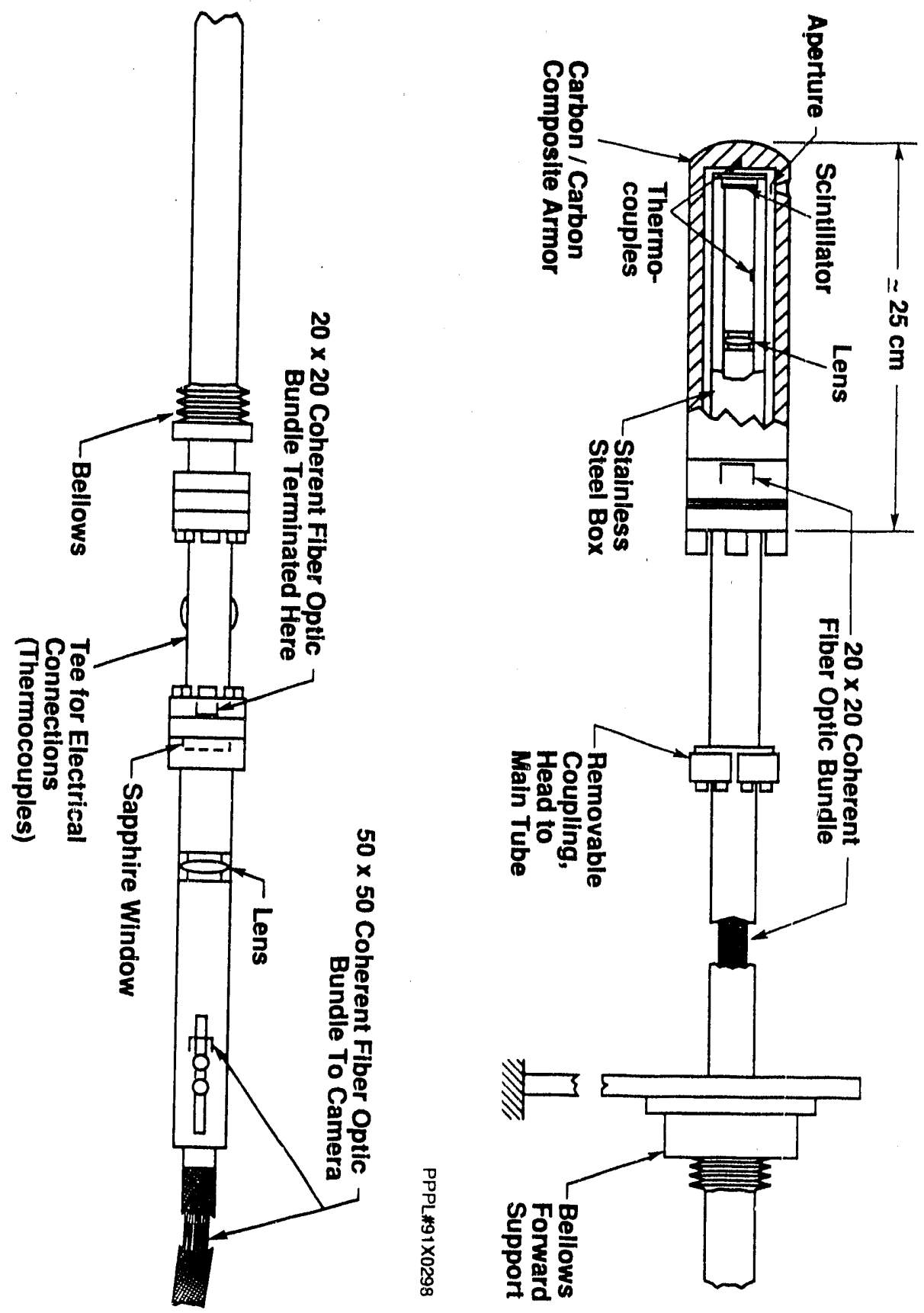

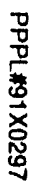

Figure 3.6: Layout of the midplane probe. 


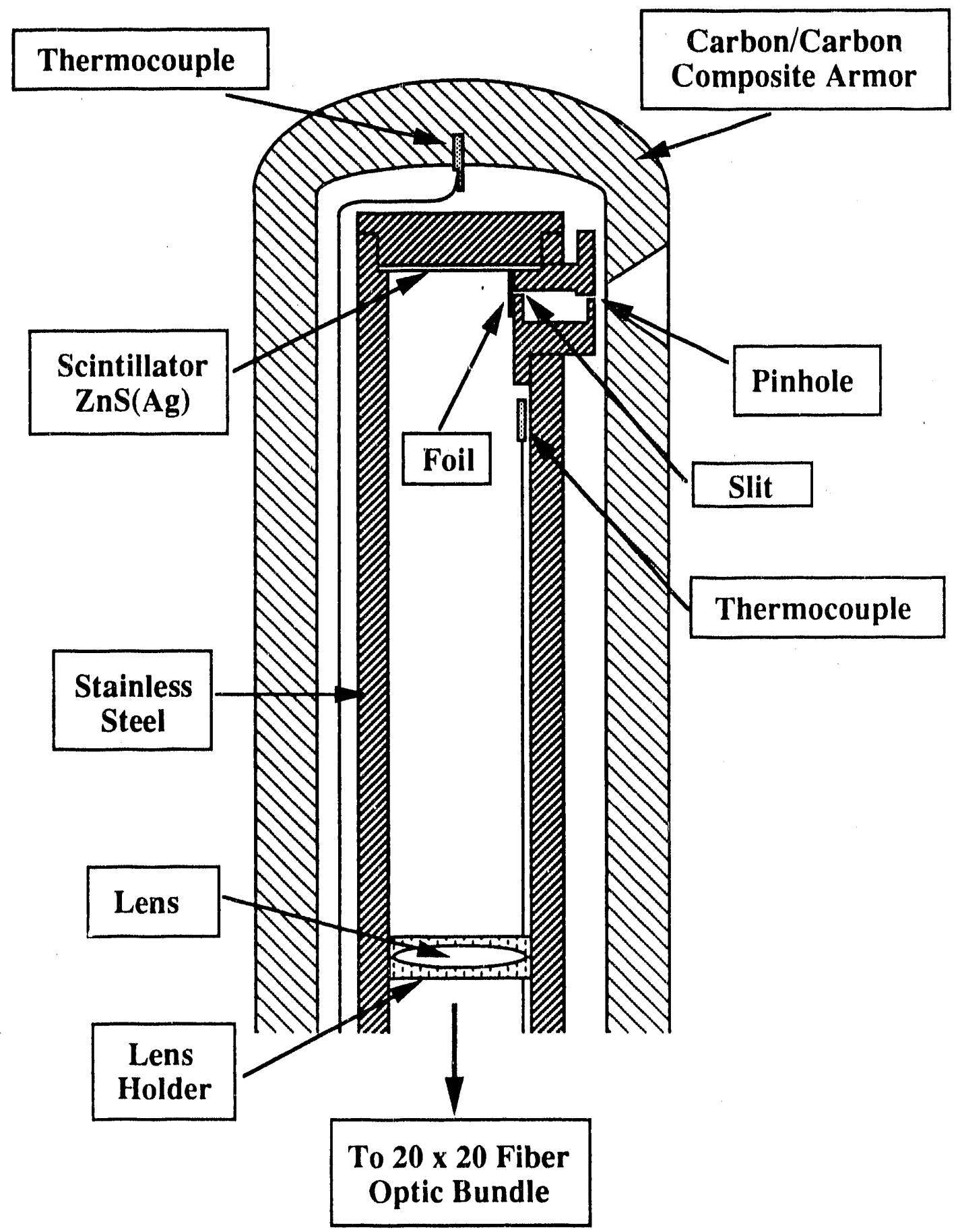

Figure 3.7: Enlarged view of the midplane probe head, showing the scintillator, apertures and optical components. All parts are drawn to scale. 


\section{TOP view}
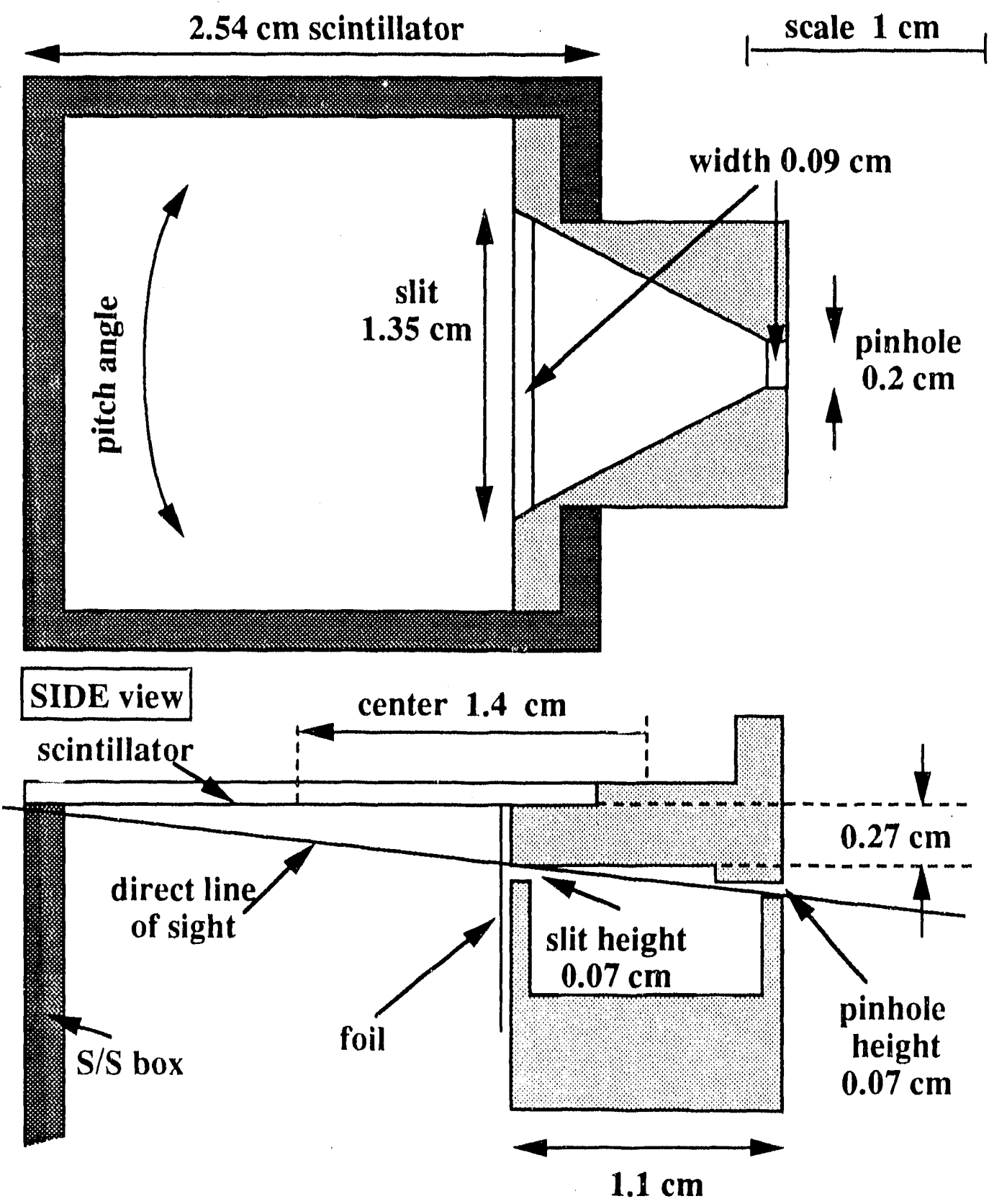

Figure 3.8: Details of the collimating apertures (top and side views) indicating the dimensions of the slit, pinhole and their relative distance. 


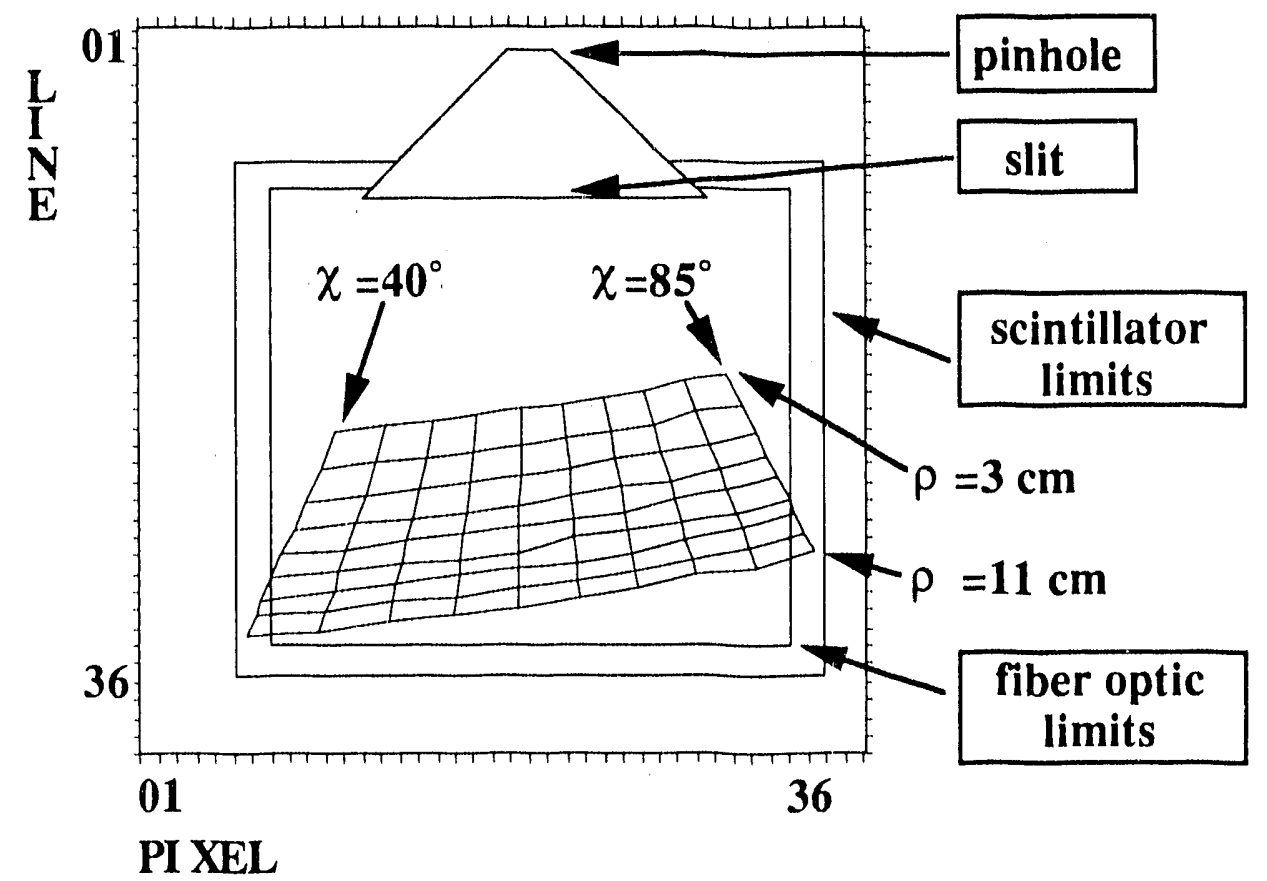

Figure 3.9: Calculated mapping of the averaged impact location (centroid) on the scintillator for different pitch angles and gyroradii for the midplane detector.

could be improved but only at the expense of less ion flux. Measurements of the optical resolution of the detector are described in Sect. 3.2.2.

In Fig. 3.7 we can also see the nonmagnetic, light-tight stainless steel case which contains and supports the various internal parts. The relatively large thickness $(0.64$ $\mathrm{cm}$ ) of the casing serves to protect the scintillator from X-rays and excess heat from the plasma. On top of the stainless steel box we used a $1 \mathrm{~cm}$ thick cylindrical 4-D weave carbon/carbon composite armor ${ }^{\dagger}$ which efficiently blocked most of the plasma heat flux to the probe head. The temperature was monitored by two thermocouples, one "floating" inside the casing next to the scintillator and another directly implanted in the carbon armor. In general, heat loads were handled very well ${ }^{100}$ as monitored by the inside thermocouple. Operating temperatures were found to be between $50^{\circ} \mathrm{C}$ and $100^{\circ} \mathrm{C}$. The temperature was found to increase less than $3^{\circ} \mathrm{C}$ per shot, except when large major radius plasmas were used or at very high repetition shot rate $\left(\lesssim 5^{\circ} \mathrm{C}\right)$. The direct contact of the external thermocouple with the armor caused an overcharge

'Making our detector the perfect Stealth Probe, since this material, made by Fiber Materials Inc., Biddeford, ME 04005, has been developed for the famous Stealth Bomber. 

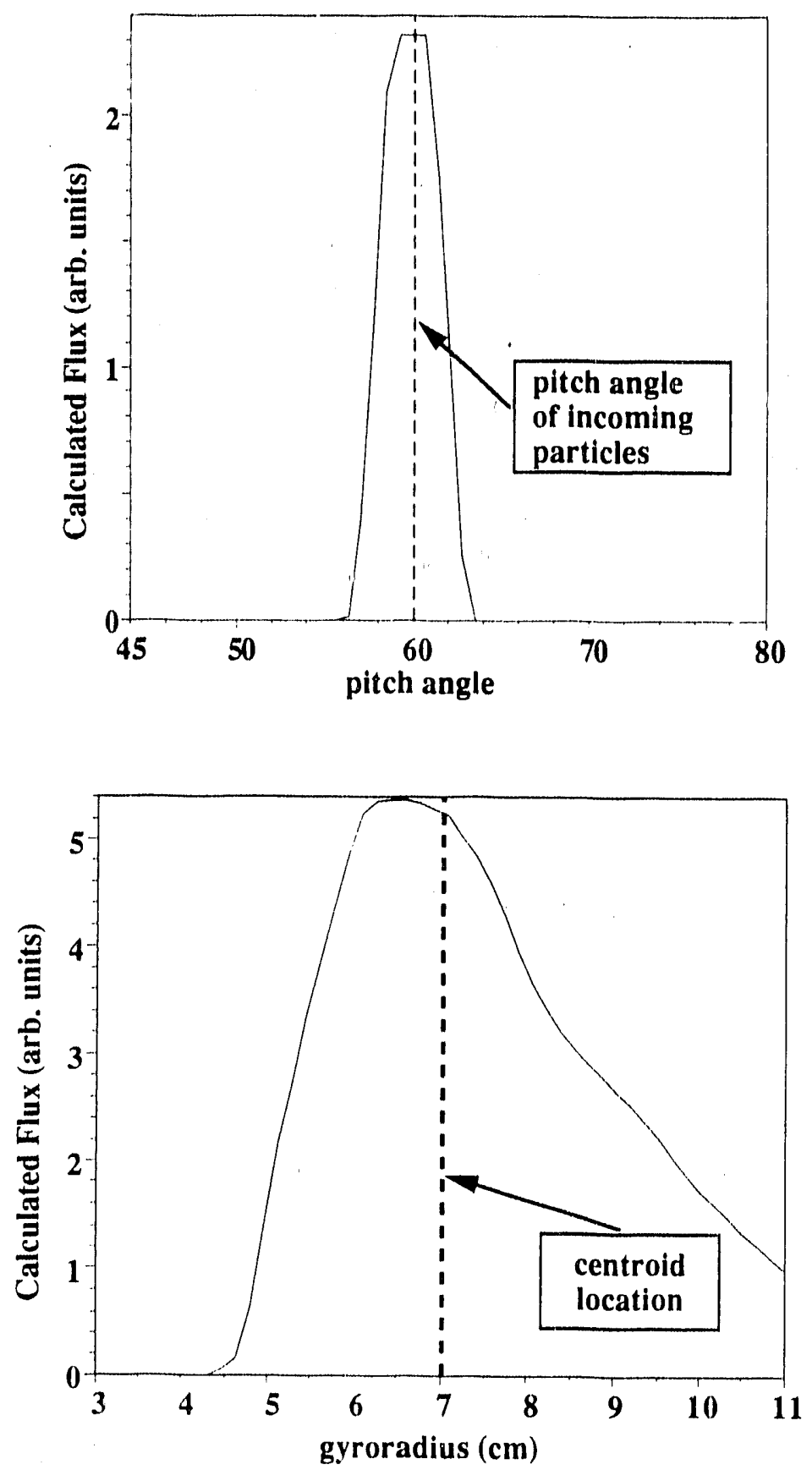

Figure 3.10: Geometrical resolution of the detector for a given pitch angle and gyroradius $\left(60^{\circ}, 7 \mathrm{~cm}\right)$; on top, the pitch angle distribution and at the bottom, the gyroradius distribution. 
of the thermocouple optical transmitter because of the potential accumulated on the probe head (estimated to be less than $1 \mathrm{keV}$ ). At that time the head was electrically insulated from the machine. At the first examination during a machine opening arc traces were found bridging over the electrical break; consequently the probe head was grounded directly through a $6 \mathrm{k} \Omega(30 \mathrm{~W})$ resistor. In any case, the orbits of the CFPs could not have been affected since the accumulated potential is small compared to the particles energy $(\sim \mathrm{MeV})$.

Inside the probe head was also placed a lens used for imaging the scintillator onto the $20 \times 20$ coherent fiber optic bundle located at the other end. All the optical parts were made of quartz (except the window, made of sapphire which has essentially the same optical properties) to minimize damage from neutron radiation. The number of fibers in the bundle (20 across) limits the possible pitch angle resolution to $2.5^{\circ}$ which is below the geometrical and optical resolutions of the detector.

\subsubsection{TFTR lost alpha detectors array}

Each detector of the poloidal array installed at the bottom uses the same basic design described in Section 3.1.1. The range in pitch angles and gyroradii is approximately the same; co-going particles between $45^{\circ}-85^{\circ}$, and between 3 to $10 \mathrm{~cm}$ in gyroradius ${ }^{90,91,94}$ can be detected. In Fig. 3.11 is shown a typical orbit $\left(I_{p}=1.4\right.$ $\mathrm{MA}, R_{0}=2.45 \mathrm{~m}, B_{T}=5 \mathrm{~T}$ ) which reaches the bottom detector (referred to as detector \#6). It is important to note that all the orbits that reach the bottom detectors are on a direct path to the wall and as such they look at lost CFPs (which is the reason why these detectors have been called the LOST ALPHA diagnostic). Data from these detectors will be used for comparison only, to stress the differences in loss mechanisms between the outer midplane and the bottom of the machine; the results shown in this thesis pertain almost exclusively to the midplane detector.

\subsubsection{Signal processing system}

At the back-end of the probe (see Fig. 3.6) is attached the coherent fiber optics bundle which transmits the image of the scintillator from the probe down to a light-tight box located in the test cell basement. The image is then recorded by a camera or by a set of photomultiplier tubes. Figure 3.12 shows the different setups used for recording the light emission of the scintillator. In the camera field of view 


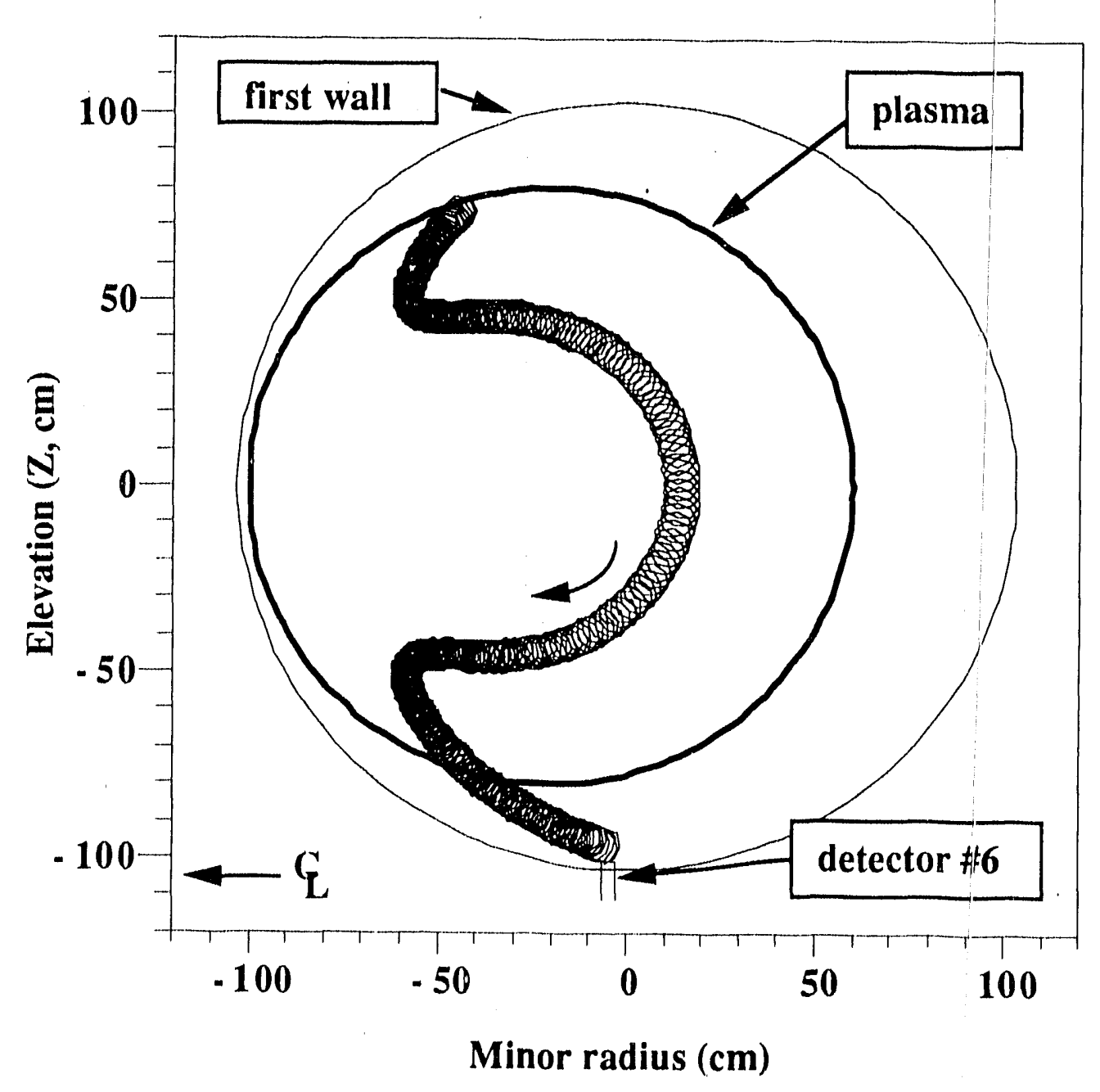

Figure 3.11: Typical trapped first-orbit lost particle trajectory (at $I_{p}=1.4 \mathrm{MA}$ ) reaching the bottorn detector $(\# 6)$. 
were placed 4 small "guide lights" (made of red LEDs) which served to monitor the linearity and the possible saturation of the camera micro-channel plate.

For all our experiments (see Fig. 3.12) we used an intensified Xybion black and white camera (model ISG-03), which was externally controlled. The maximum frame repetition rate possible is $16.6 \mathrm{~ms}$ (limiting the time resolution in that mode). The different levels of light coming from the scintillator were handled by changing the gate time (practically from $20 \mu \mathrm{sec}$ to $15 \mathrm{msec}$ ). Both the gate and the trigger times were set from the control room through CAMAC modules. The camera output was then sent through fiber optic cable to the control room to a VCR (for backup recording) and to a IBM-PC based acquisition system. The system is based on a $4 \mathrm{MB}$ video frame grabber ${ }^{\ddagger}$ which can digitize 42 frames. Each frame is then compressed $3 \times 3$ before being transferred to the VAX. Shown in Fig. 3.13 is an example of a view of the scintillator after compression. The $x$-axis corresponds to increasing pitch angle and the $y$-axis to decreasing gyroradius. The aperture is located near the center top of the image. Also shown are the limits of the scintillator.

When good time resolution was necessary (for looking at fast events like MHD activity and sawteeth) a set of $1 \mathrm{~mm}$ diameter plastic optic fibers ${ }^{\mathfrak{f}}$ were proximity coupled to the long coherent fiber optic bundle. A total of 24 photomultiplier tubes was available to look at specific pitch angles and gyroradii on a combination of detectors. The voltage of the photomultiplier tubes is also remotely controlled but was set ty'pically at $-1000 \mathrm{~V}$. Signals are then sent to preamplifiers", digitized by a set of ADC"l and archived on the VAX. The signals were directed to a set of 31 digitizers, 15 "fast" (between $200 \mathrm{~Hz}$ and $500 \mathrm{kHz}$ ) and 16 "slow" (between $100 \mathrm{~Hz}$ and $20 \mathrm{kHz}$ ).

So far the two systems have not be used simultaneously for one detector but future plans call for to use of the beam-splitter to view the detector images from both the carnera and the photomultiplier tubes.

\footnotetext{
${ }^{\dagger}$ Epix, North Brook, IL 6006'

${ }^{\S}$ ESKA Extra, Mitsubishi

"model A-1, Thorn EMI, Gencom Inc, Fairfield, NJ 07006

" Analog-Digital Converters, model TFTR-10C6-H908, H907B and TFTR-10C1-H907
} 


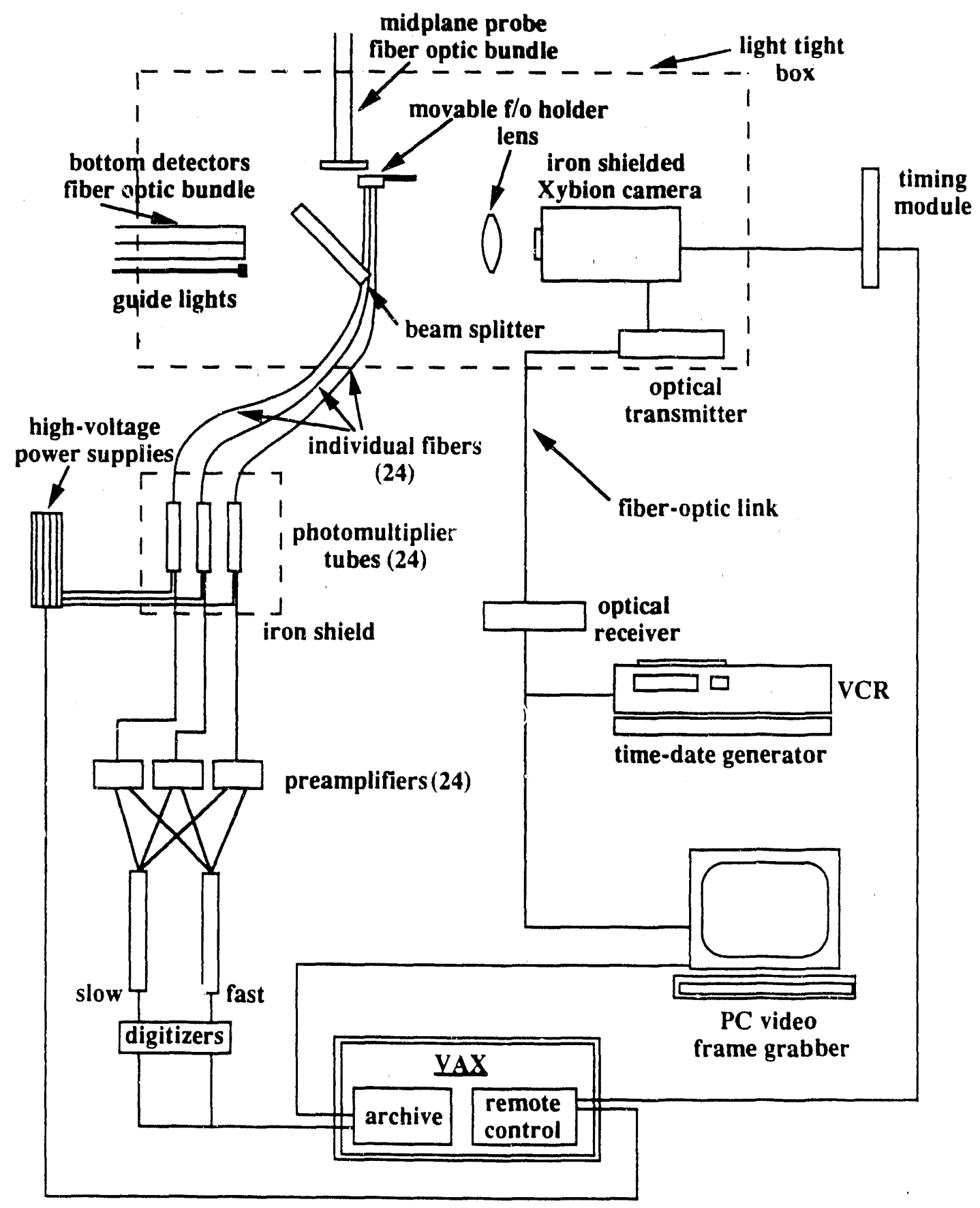

Figure 3.12: Schematic diagram of the light recording system for the detectors indicating the parallel systems of camera and photomultiplier tubes. 


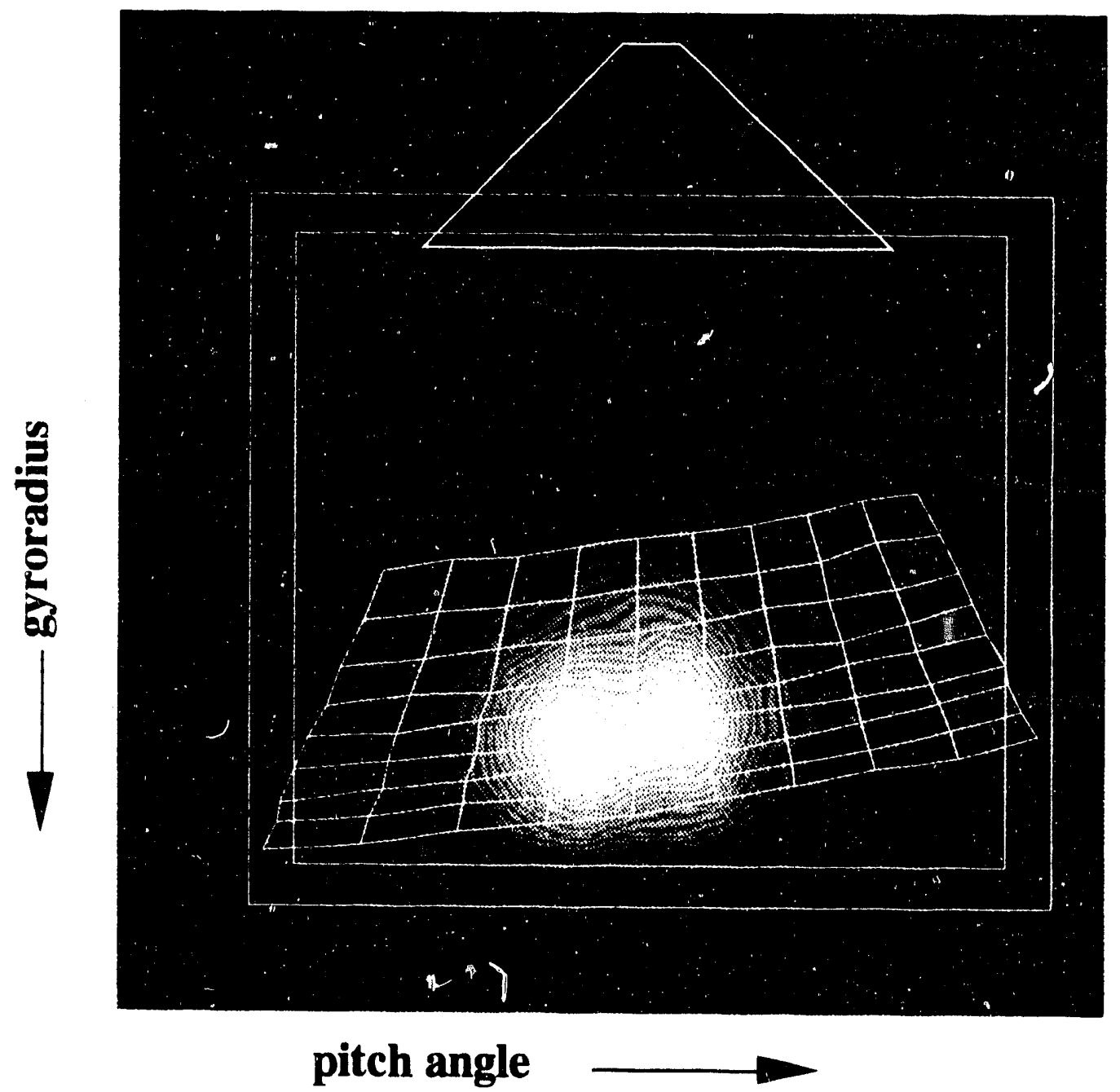

Figure 3.13: Processed image of the scintzllator after compression, indicating the :mission light intensity. The r-aris corresponds to increasing pitch anqle and the $y$-axis to decieasing gyroradius. Also indicated are the scintillator limits and the impact centroids as shoun in Fig. 3.9. The pinhole and slit are located at the center of the top sade of the scinizllator. 


\subsection{Calibration}

In every experiment the most arduous and also the most important task lies in the interpretation of the results. Although many important results can be obtained by relative measurements (i.e. escaping flux vs. plasma current, pitch angle, plasma position, etc.) the ultimate answer for this experiment (in given conditions) is the actual number of particles lost. Simply put, for a given amount of light recorded from the scintillator, one needs to infer through calibration and calculations how good is the confinement of CFPs. To do so, a systematic study of the diagnostic and the physics involved is necessary.

This diagnostic has been divided in two relatively independent parts which facilitated the task. The first part, seemingly simple, consists in the scintillator itself. Although used for decades now (Giesel discovered it at the end of the $19^{\text {th }}$ century and Rutherford used it to study alpha particles at about the same period) the $\mathrm{ZnS}(\mathrm{Ag})$ is not completely understood, in fact full books have been written on those matters. ${ }^{102,103,104}$ We will attempt a characterization of the scintillator, at least for our purpose. The second part, consists of the optical coupling between the scintillator and the recording medium. Although consisting of many parts, it is a more straightforward task but definitively, equally delicate. Finally, to complete the process, there is the interpretation of the results, mainly a numerical problem. All those aspects will be described in the next sections although many details will be left for the appendices.

One major obstacle prevented a complete and direct calibration from the vacuum vessel system. This problem, important in D-D operations, is the absence of radioactive isotopes emitting high energy protons and tritons. Consequently, one needs to calibrate the scintillator inside an auxiliary D-D fusion generator. This will be the subject of the next section.

\subsubsection{The $\mathrm{ZnS}(\mathrm{Ag})$ scintillator}

The process of scintillation involves solid state physics. ${ }^{105,106}$ However, the basic process of scintillation can be explained in a few words. In pure and perfect crystals (with no impurities and perfect crystal structure) the diagram of the electron energy band structure exhibits only a forbidden region in between the valence and the conduction bands. In the valence band electrons are tied to the lattice site whereas 


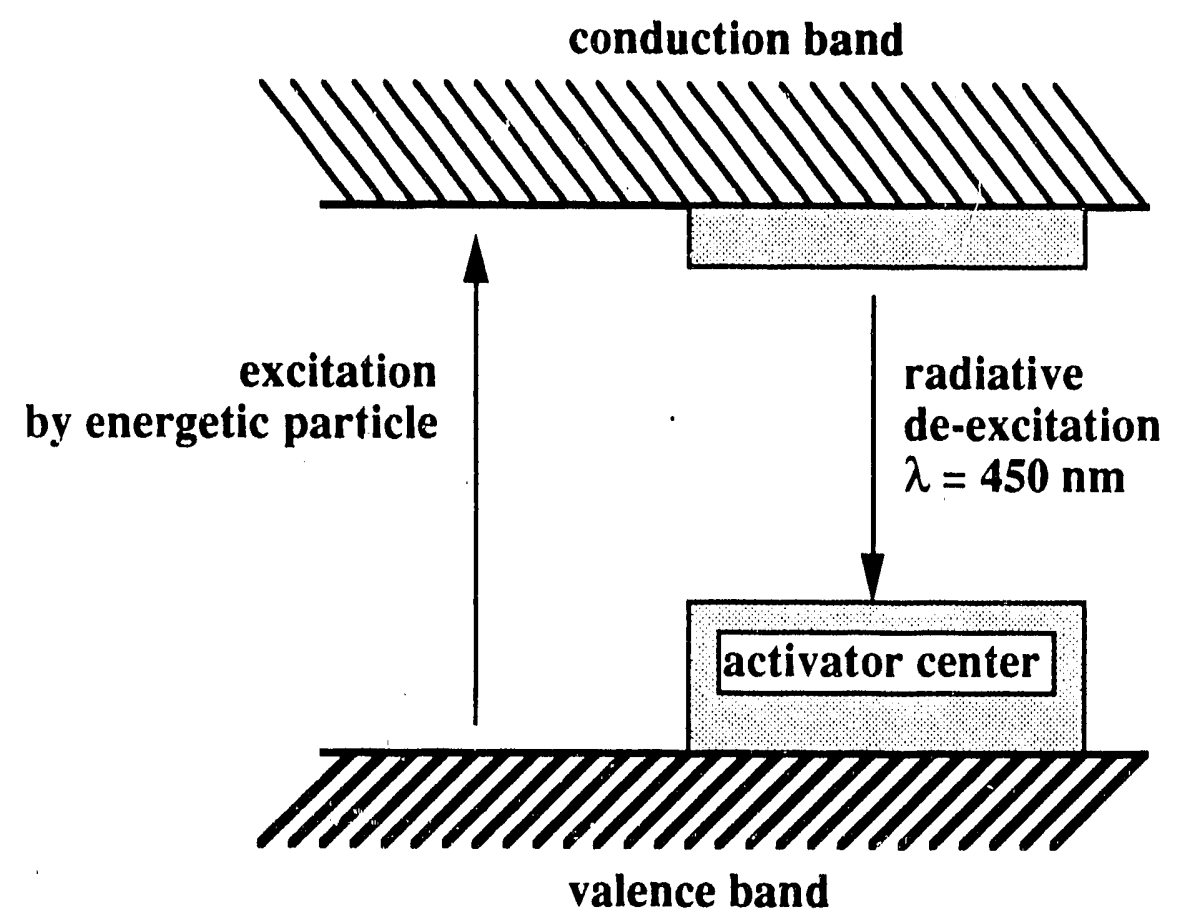

Figure 3.14: Physical picture of the scintillation process. Added impurities facilitate the radiative decay of excited states by partly filling the gap in the forbidden region.

in the conduction band they are free to move within the crystal. Ionization radiation (i.e. $\mathrm{MeV}$ ions) would kick electrons into the conduction band thus forming a electron-hole pair. For pure and perfect crystals the inverse process (de-excitation) is improbable (thus very slow) and the emitted radiation is likely to be out of the visible region. In the case of activated crystals (in our case by silver, $\mathrm{ZnS}(\mathrm{Ag})$ ) intermediate levels appear in the forbidden region (see Fig. 3.14), the probability of radiative de-excitation is greatly enhanced and if the activator is carefully chosen, the wavelength would be in the visible. The picture can be complicated by the presence of more than one radiative transition, i.e. different wavelengths. In $\mathrm{ZnS}(\mathrm{Ag})$ scintillation is very largely dominated by a transition occurring at $450 \mathrm{~nm}$, in the blue region of the visible spectrum.

Another important characteristic of the scintillator is its decay time constant, which is in fact constituted of two components for the $\mathrm{ZnS}(\mathrm{Ag})$. It has been observed ${ }^{95}$ that for a single pulse, the scintillator light output decays back to $10 \%$ of the maximum level in less than $10 \mu \mathrm{sec}$. However, a longer decay constant has been found in light flux mode (by opposition to the single pulse mode) with a few percent $(<3 \%)$ of the 
maximum flux level remaining after $10 \mathrm{msec}$.

Presently the $\mathrm{ZnS}(\mathrm{Ag})$ is not grown in large crystals (usual size is $\sim 10 \mu \mathrm{m}$ ); consequently the scintillator is actually made of a powder composed of many tiny crystals. This has the principal consequence that the scintillator is opaque to its own light, the emission light is reabsorbed and redistributed within the scintillator; the effects of opacity will be discussed below.

In Fig. 3.15 is shown the range of pertinent energetic particles in the scintillator according to their energy as taken from tables of stopping powers in $\mathrm{ZnS}^{107,108}$ (see App. B.3). Note the relatively long range of the $3 \mathrm{MeV}$ proton compared to the 1 $\mathrm{MeV}$ triton. These non-linear energy and particle dependencies are the fundamental difficulties of this calibration process. The combination of range and opacity, as discussed above, were the main reasons for the choice of a very thin scintillator, around $10 \mu \mathrm{m}$. This choice had also the definite advantage of eliminating gamma and neutron scintillation, leaving the $\mathrm{X}$-rays (originating from the plasma and from reflections on the walls) as a noise concern. As mentioned in Section 3.1.1 the problem was eliminated by shielding the scintillator with at least $3 \mathrm{~mm}$ of stainless steel. ${ }^{91}$

The other major concern with the $\mathrm{ZnS}(\mathrm{Ag})$ is its strong temperature dependence ${ }^{95}$ as shown in Fig. 3.16 . Here, the light response to ${ }^{244} \mathrm{Cm}$ alphas was monitored while the scintillator was heated using hot plates. The scintillator response is relatively flat until $\sim 150^{\circ} \mathrm{C}$ above which it is plummeting. This quenching effect is still not completely understood ${ }^{102,103}$ and we must avoid that regime (and carefully monitor the temperature) or use a more thermally stable scintillator. The scintillator is nevertheless not damaged below $500^{\circ} \mathrm{C}^{95}$; when returned to normal temperature the light response returns to its original value. With that restriction, detectors have to be well protected against the plasma heat flux or be actively cooled, both of which are cumbersome and/or difficult. Other scintillators with better thermal handling capabilities have been suggested (for example $\mathrm{ZnS}(\mathrm{Cu})$ |type $\mathrm{P}-31$ ], $\mathrm{Y}_{3} \mathrm{Al}_{5} \mathrm{O}_{12}(\mathrm{Ce})$ [type $\mathrm{P}-46])$ but they all have a relatively low light efficiency compared to the $\mathrm{ZnS}(\mathrm{Ag})$ (type P-11]).

As mentioned above the first step in the calibration of the diagnostic lies in the scintillator itself. Here is the list of items we wish to examine in this process:

- Absolute calibration: light flux vs. particle flux

- Light flux vs. particle energy 

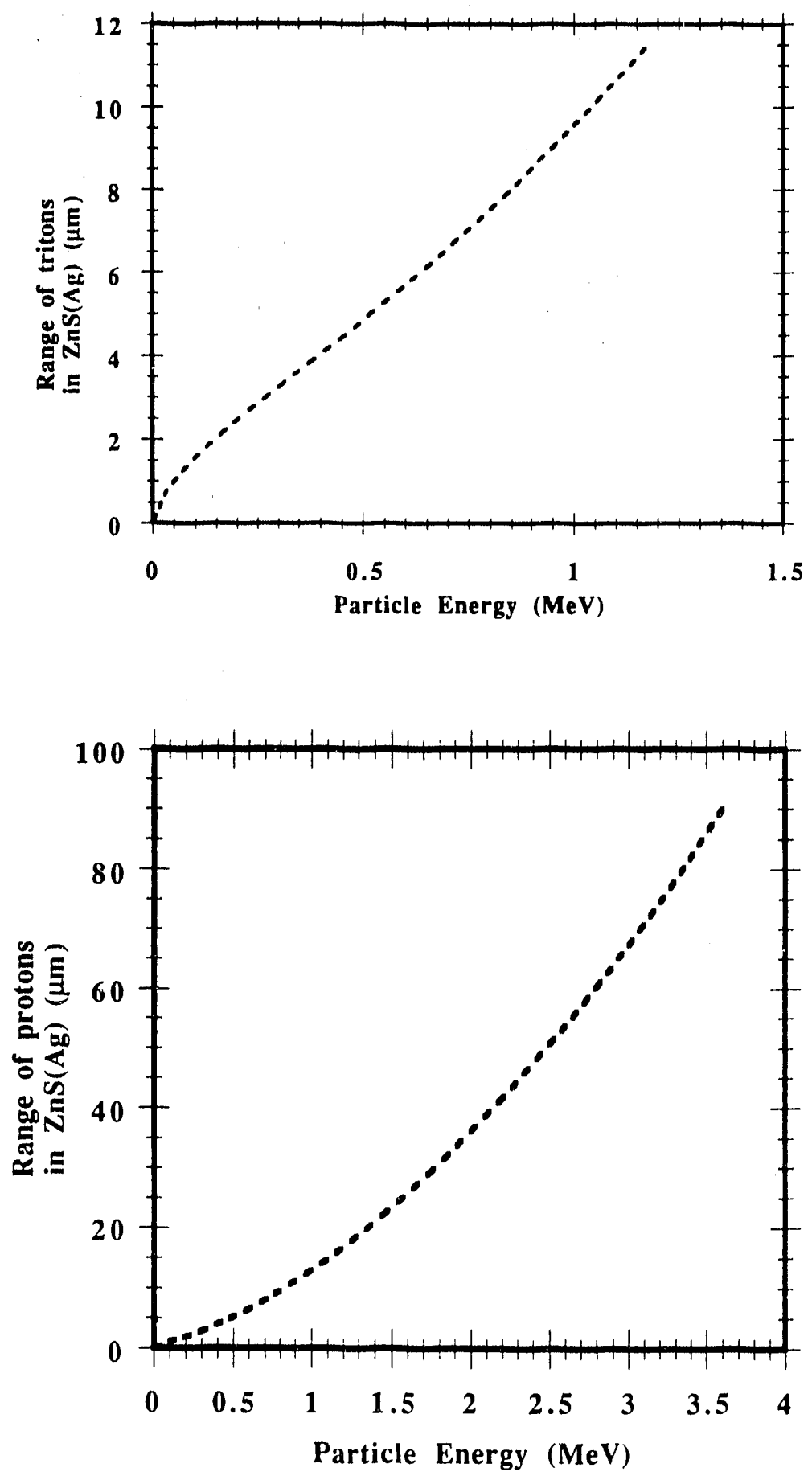


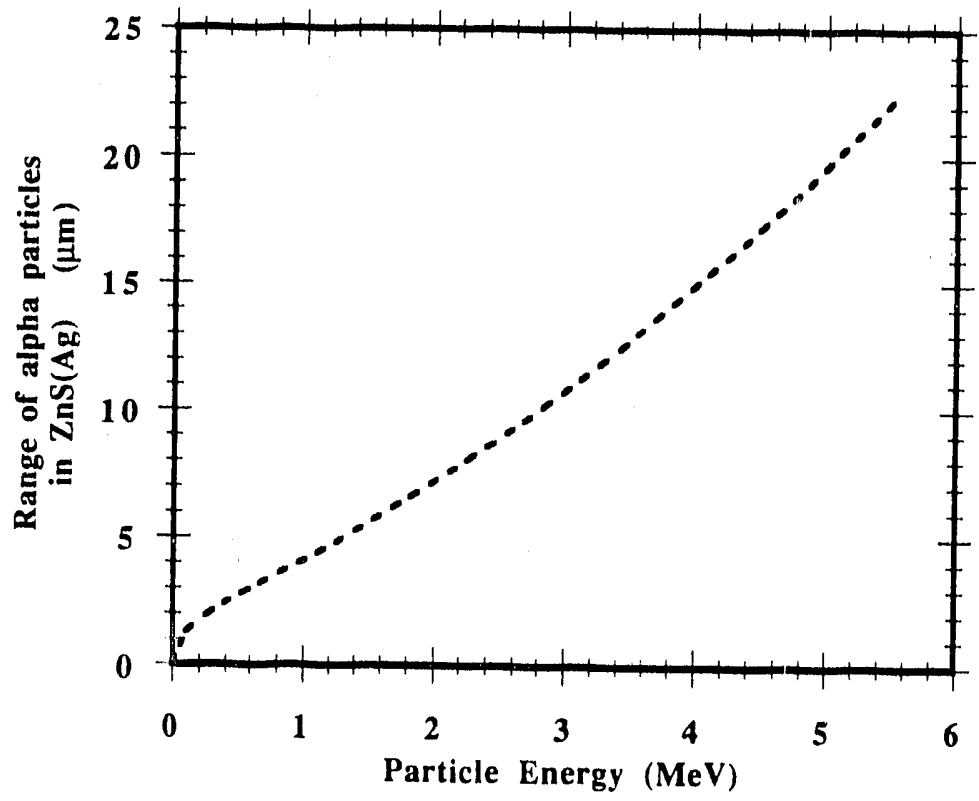

Figure 3.15: Particle ranges in $Z n S(A g)$ for the triton, proton and alpha versus their incident energy. 


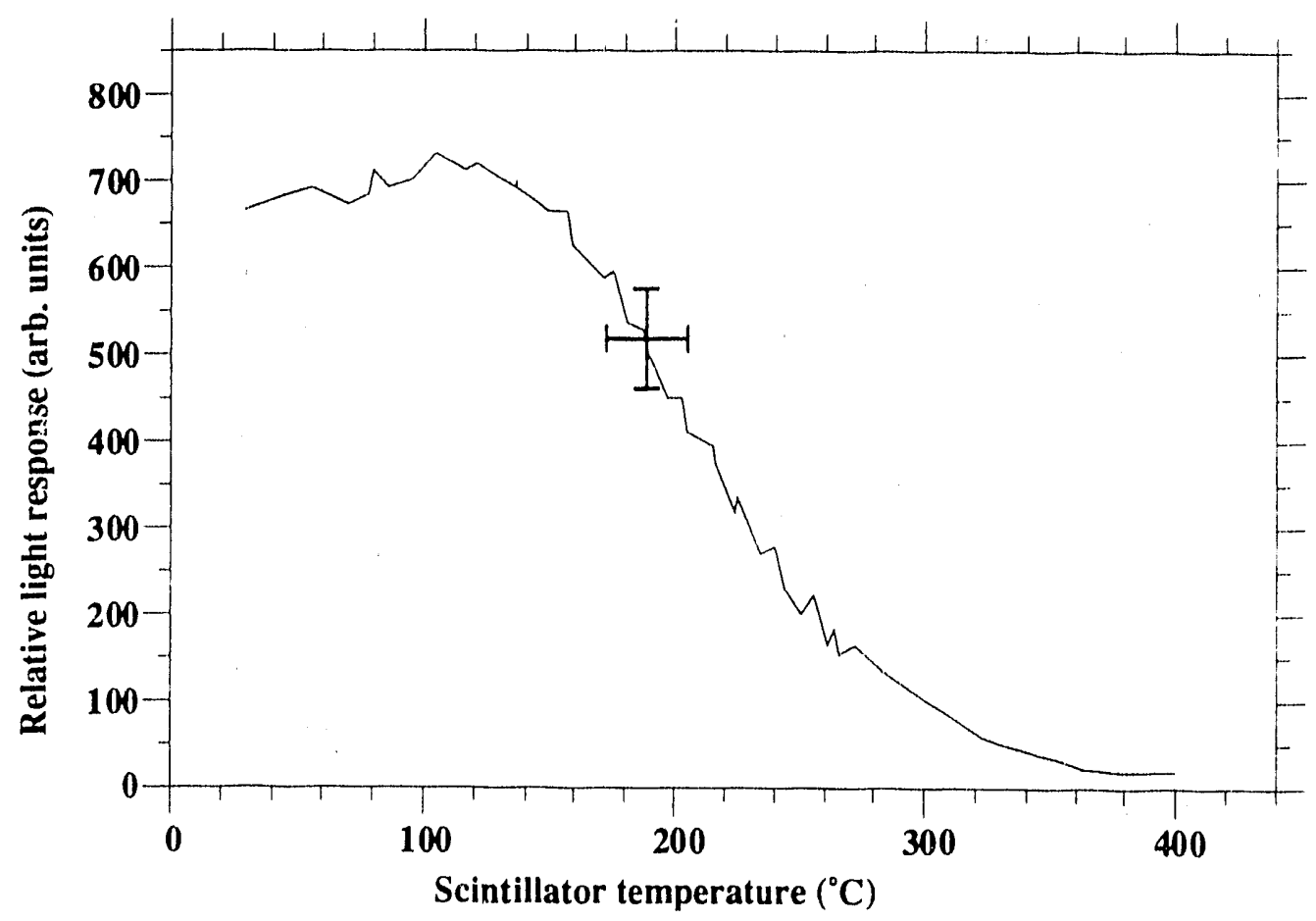

Figure 3.16: Temperature Uependence of the light emission for the $\mathrm{ZnS}(\mathrm{Ag}){ }^{95}$ 
- Light flux vs, particle type

- Light flux vs. particle angle of incidence.

- Scintillator linearity and saturation levels

Most of the above items have been studied extensively whereas some (i.e. saturation) are still under investigation. As we mentioned previously the absence of isotopes for the D-D CFPs necessitated the use of a "fusion" generator using a (oockcroft-Walton ion beam. Another difficulty lies in the very short range of those $\mathrm{MeV}$ ions in air necessitating a vacuum chamber for an effective calibration.

In Fig. 3.17 is shown the setup for the scintillator calibration. On one side is the ion beam itself, capable of voltages up to $150 \mathrm{kV}$ (and with some simple modifications up to $200 \mathrm{kV}$ ) and beam currents up to a few milliamperes. Magnets could be put in the accelerator section in order to remove half and third energy components but were not used in our experiment for better target loading. The target chamber, with a diameter of $45 \mathrm{~cm}$ and a depth of $15 \mathrm{~cm}$, contained the target and the different detectors. The target was made of titanium (a $1 \mathrm{~cm}$ diameter, $0.6 \mathrm{~cm}$ thick "butt") and simply attached to a movable holder without active cooling. A target made of zirconium was also made in case the deuterium loading in titanium was not good enough. Both materials (and also palladium) were chosen for their good deuterium retention. The detectors were located close to the target (typically $10 \mathrm{~cm}$ or so) in order to maximize the count rate. The silicon barrier detector (or surface barrier diode, SBD) was installed next to the scintillator for flux and energy calibration of the CFP source. The SBD was itself energy calibrated by using an ${ }^{241}$ Am alpha source with a $4.5 \pm 0.3 \mathrm{MeV}$ peak energy (after an internal gold foil). Next to the SBD, we put the scintillator facing the target at an angle of $15^{\circ}$ to $20^{\circ}$, simulating the actual angle of incidence found in the actual detectors (see Fig. 3.7). A photomultiplier tube was installed very close to the scintillator in order to maximize photon recording. Signals were then transmitted by feedthroughs to preamplifiers located outside the chamber. Details of the electronics and experimental conditions are described in Appendix B. The energy of CFPs was changed by simply putting aluminum foils of different thicknesses (from 0.8 to $59 \mu \mathrm{m}$ ) in front of both the SBD and the $\mathrm{ZnS}(\mathrm{Ag}$ ).

Because of the relatively low production of CFPs the measurements were made in the pulse count mode (typically $10^{3}$ counts $/ \mathrm{sec} / \mathrm{cm}^{2}$ ) as opposed to the flux counting 


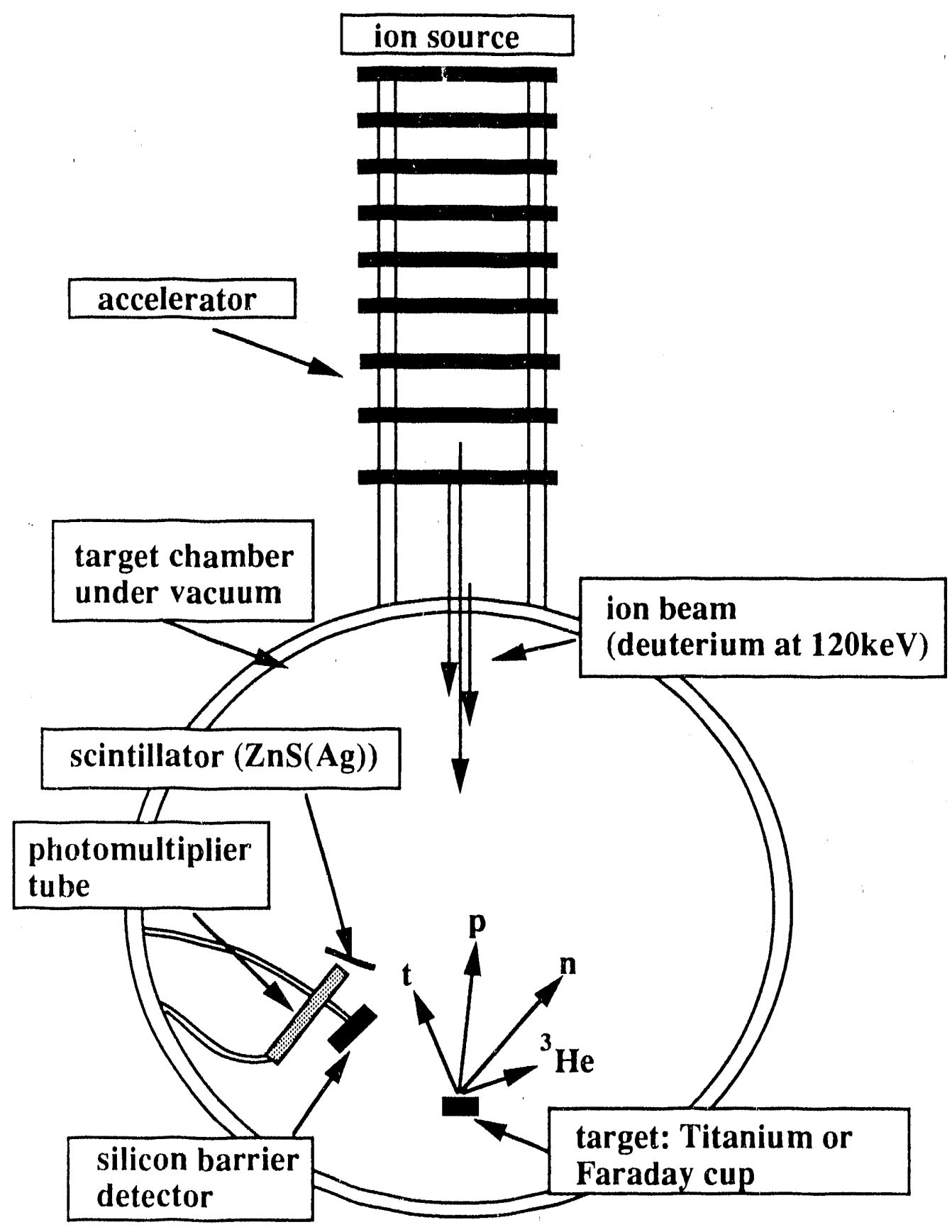

Figure 3.17: Schematic diagram of the experimental setup for the scintillator calibration. 
mode (typically $10^{8}$ counts $/ \mathrm{sec} / \mathrm{cm}^{2}$ ) used in the actual set-up in TFTR. Nevertheless it was believed that for the items listed above, the results would remain valid in both conditions (except, naturally, for the possible saturation effects at very high flux). Shown in Fig. 3.18 is a typical pulse height spectrum for the SBD and for the photomultiplier tube looking at the scintillator. In this case, a $2 \mu \mathrm{m}$ aluminum foil was used in front of the detectors. Attention needs to be drawn to the SBD spectrum where the effect of the Doppler shift on the particles energy is visible. Because the detectors are located at an angle of approximately $45^{\circ}$ on the beam side, the triton energy would be degraded by up to $25 \%$ (and somewhat less for the proton ${ }^{109,110}$ ). The $0.8 \mathrm{MeV}{ }^{3} \mathrm{He}$ is stopped by the thin aluminum foil except at the thinnest foil $(0.8 \mu \mathrm{m})$ where it is barely visible on the SBD energy spectrum.

The other graph shows the scintillator photon pulse height spectrum. Because the scintillator responds only to energy deposited, and since the $\mathrm{ZnS}(\mathrm{Ag})$ is opaque to its own light, it was not possible to separate the pulse height spectra of the tritons and protons. The scintillator granular structure is also an important factor in the emitted pulse height spectrum. The small $\mathrm{ZnS}(\mathrm{Ag})$ crystals form only a very thin layer of $1-2$ crystals thick. In those circumstances the deposition of energy varies according to the particle effective range in the different small crystals. Consequently, the difference in light response from the two particles was uncovered by using their different range in aluminum. By using thicker aluminum foils (thus stopping the triton but not the proton), it is possible to isolate the proton contribution.

The first two items in our calibration list can be studied together. The absolute calibration of the scintillator will be based on the light response from a known source of alpha particles. The light emission from alpha particles (from the ${ }^{241}$ Am source) will be compared to the one from protons and tritons and to an absolutely calibrated light source. We will then have the absolute value of the illuminance of the $\mathrm{ZnS}(\mathrm{Ag})$, $10 \mu \mathrm{m}$ thick, from the exposure to high energy particles in a geometry similar to the one used in the detectors.

In Fig. 3.19 are shown the energy dependencies of the scintillator to protons, tritons and alphas (all three curves with the same vertical scale). The absolute illuminance of the $\mathrm{ZnS}(\mathrm{Ag})$ is given as a function of the particle energy before a $3 \mu \mathrm{m}$ foil as used in our detectors. The experimental curves are compared with a very simple model calculating the amount of energy deposited in the scintillator. All the model curves are vertically positioned so that for a particle with no energy after the foil 


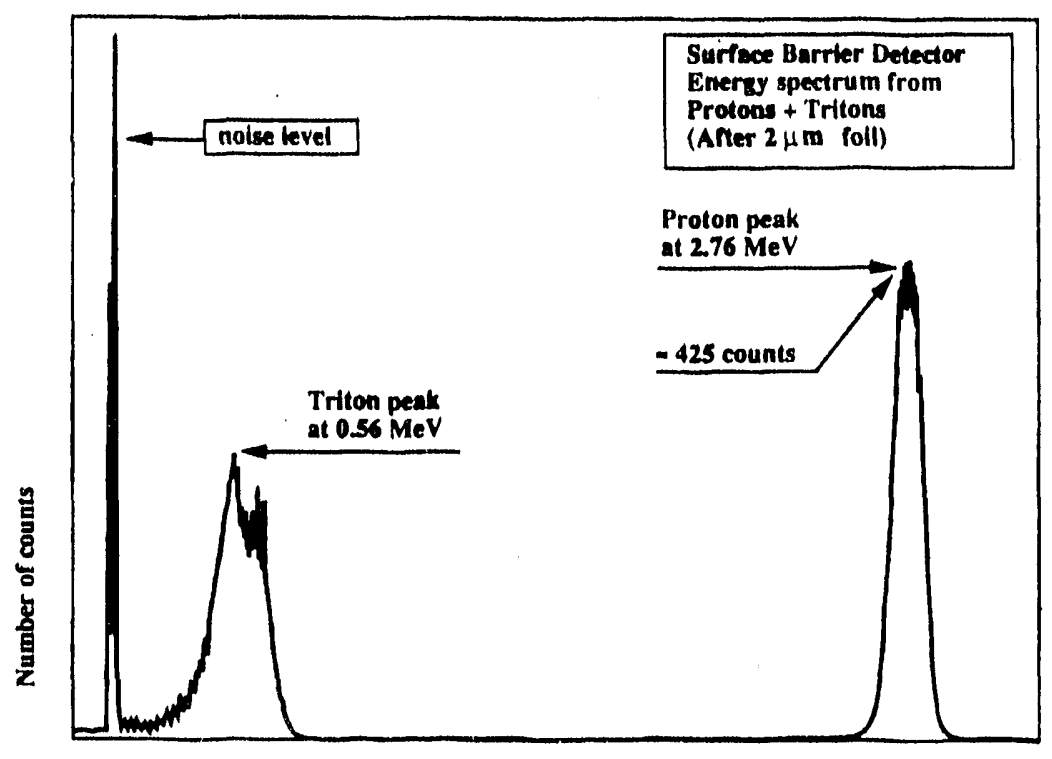

Particle enery (MeV)

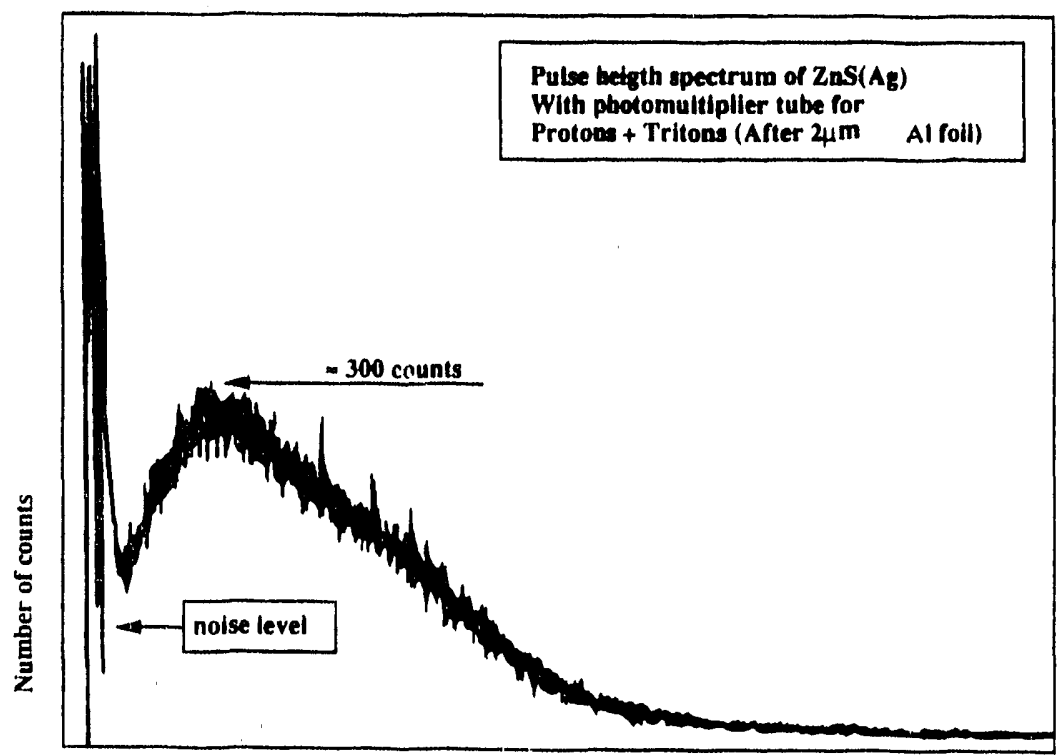

Pulse heigth (arb. units)

Figure 3.18: Typical pulse-height spectra for the silicon barrier detector (top) and for the photomultiplier tube(bottom) coupled to the scintillator. In this case, a $2 \mu \mathrm{m}$ aluminum foil was used in front of the detectors. Large levels at low pulse heights are due to detector noise. 
the scintillator response would be zero, and are also normalized at the highest energy point. The three curves give also the ratio of the light response of the scintillator for different particles at their birth energy. For example, a $3 \mathrm{MeV}$ proton gives, in that geometry, $1.5 \pm 0.2$ more light than a $1 \mathrm{MeV}$ triton, whereas the $3.5 \mathrm{MeV}$ alpha would give approximately $8.0 \pm 2.0$ times more. Those energies correspond to the particles energy before they go through the detector's $3 \mu \mathrm{m}$ aluminum foil.

This result is very important, it implies that $\sim 60 \%$ of the light signal are due to protons (and so $40 \%$ to tritons) contrary to what was previously estimated. ${ }^{91,93,1(0)}$ The reasons for such a ratio are multiple. First, after the $3 \mu \mathrm{m}$ Al foil installed at the slit, the triton energy is only $\simeq 0.7 \mathrm{MeV}$, whereas the proton energy is still close to $3 \mathrm{MeV}$. Then the particles impact on the scintillator at an angle of $15^{\circ}$ to $20^{\circ}$. At that angle the scintillator has an effective thickness of 30-40 $\mu \mathrm{m}$, compared to the 10 $\mu \mathrm{m}$ seen by a normally incident particle. In that situation the $3 \mathrm{MeV}$ proton would deposit approximately $2.2 \mathrm{MeV}$ of its energy and the triton all its $0.7 \mathrm{MeV}$. However, the energy deposited by the proton would be localized mainly in the back side of the scintillator (away from the photomultiplier tube or camera) contrary to the triton energy, mainly deposited very near the surface. As the scintillator is opaque to its own light a sizable fraction of the proton produced light would not reach the front surface, explaining at least qualitatively the ratio that we obtained. In the case of an alpha particle, it is stopped very quickly in the scintillator because of its double charge. However, when we compare the ratio of light flux from alphas to tritons with their ratio of energy after the foil (2.9 MeV alpha to $0.7 \mathrm{MeV}$ triton) we see that the factor of 8 found above is higher that what we would expect from just energy considerations although even though they have approximately the same range in $\mathrm{ZnS}(\mathrm{Ag})$. It is unclear at this point if this is evidence of different scintillation efficiencies for the triton and the alpha particle. One final consequence of this measurement (important for the eventual D-T operations), is that for the same number of alpha particles, we can expect a factor of $\sim 3$ increase in signal over protons and tritons combined.

The opacity has been also measured approximately by using the alpha source and two identical scintillators. ${ }^{95}$ The light flux from the normally incident alphas was measured with a photomultiplier tube. In the second pari of the experiment, the second scintillator was inserted between the first scintillator and the photomultiplier tube, absorbing or diffusing part of the emitted light. It was found that only $30 \%$ to $40 \%$ of the light was transmitted through the second scintillator. This crude 

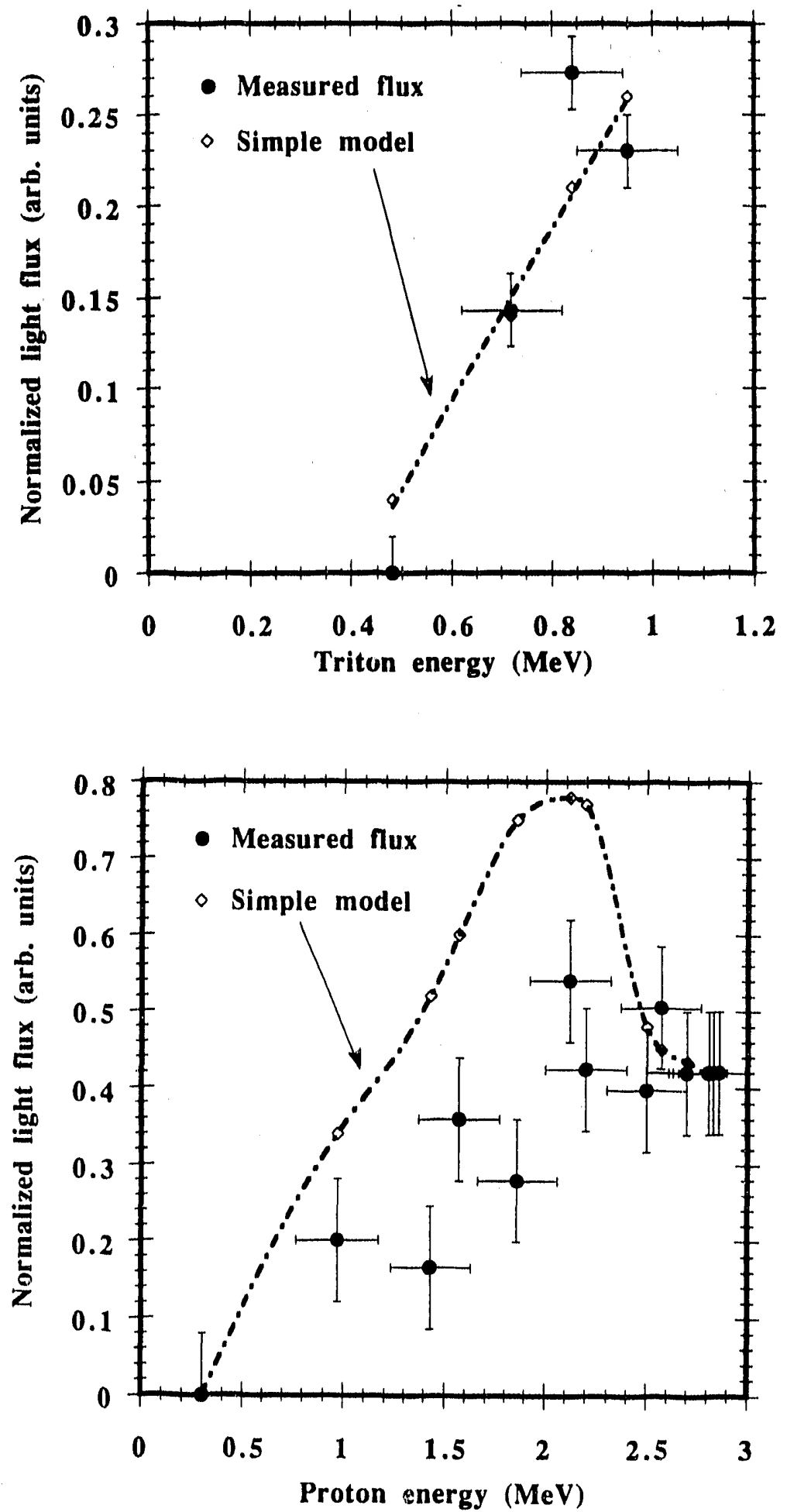


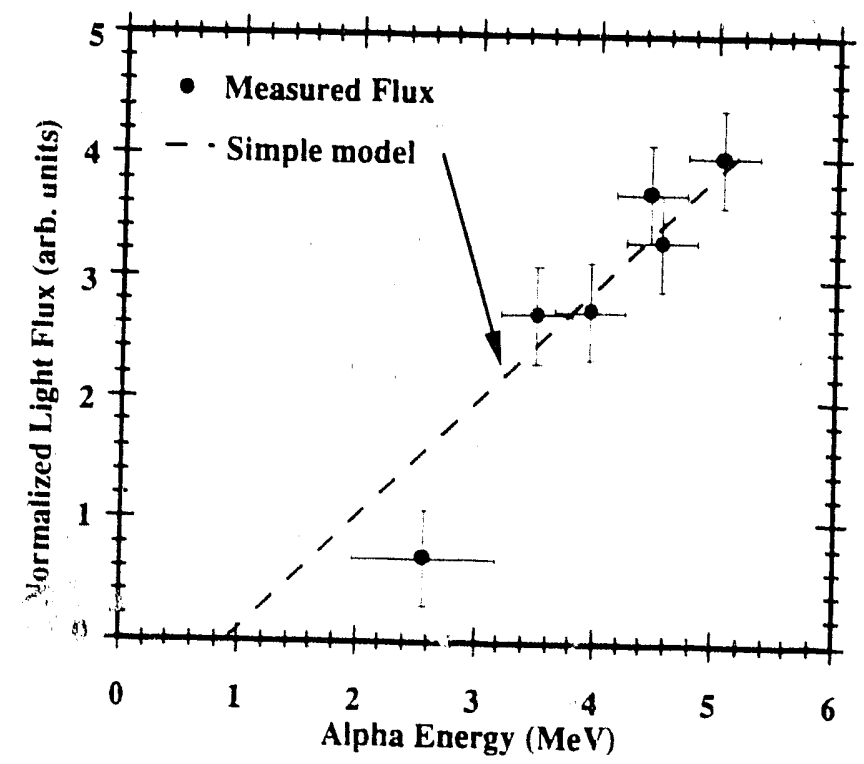

Figure 3.19: Measured and calculated energy dependence of the scintillator for tritons, protons and alphas. The three experimental curves are based on the same vertical scale. 
measurement served only to estimate the order of magnitude of the opacity. A more detailed analysis would be necessary for modeling properly the results shown above.

The effect of opacity can also be seen in the ratio of emitted light depending on the viewing side. If we define the front of the scintillator as the side with the crystals and the back, the one with the quartz substrate, we found that, with $4.5 \mathrm{MeV}$ alphas impacting at $45^{\circ}$, the front side was $1.8 \pm 0.3$ times brighter than the back one (see also App. B.4. Another factor to consider is the particle angle of incidence. We show in Fig. 3.20 the angular dependence of the light emission for the alpha source, one curve shows the results corrected for the solid angle and another the results without." However, we can not, at least at this stage, generalize these results to other energies and particles. This dependence was obtained with a view from the back of the scintillator with $4.5 \mathrm{MeV}$ alpha particles. The combination of the effects due to different types of particles, their different energy deposition and the scintillator opacity prevent such a generalization.

\subsubsection{Optical calibration}

The second step in the calibration of the detectors (the three at the bottom and the one at the midplane) consisted in replacing the scintillator by a known source of light. The level of light was recorded by the camera and digitized by the PC board with the same setup used during plasma operations. The light source, made of an halogen lamp, a white diffuser ${ }^{* *}$, and a blue filter ${ }^{\dagger \dagger}$, was compared on the bench to the luminosity of the scintillator with the ${ }^{241}$ Arn alpha source. The direct use of the alpha source in the vacuum vessel was not feasible because of its relatively low activity and also because of radioactive materials handling difficulties. We found that the light source was $3.3 \pm 30 \%$ brighter than the scintillator with the alpha source. Here the source was placed directly next to the scintilator, with the alphas impacting with a quasi normal incidence. The level recorded by the camera for the scintillator and the source had also to be corrected. by a factor of $1.7 \pm 55 \%$, since the camera looked at the back side of the scintillator. The source has been found (see A pp. B.4) to produce $2.1 \times 10^{6} \pm 25 \%$ alphas $/ \mathrm{cm}^{2} / \mathrm{sec}$. By combining these factors we found that for a recorded light leve! of $10 \%$ from the camera (of the camera full

- Plexiglass W-2447, 1/4" thick, excellent for making a lambertian (isotropic and uniform) source. ${ }^{111}$

t'Kodak wratten gelatin \#47 A (at $\lambda=450 \mathrm{~nm}$ ). 


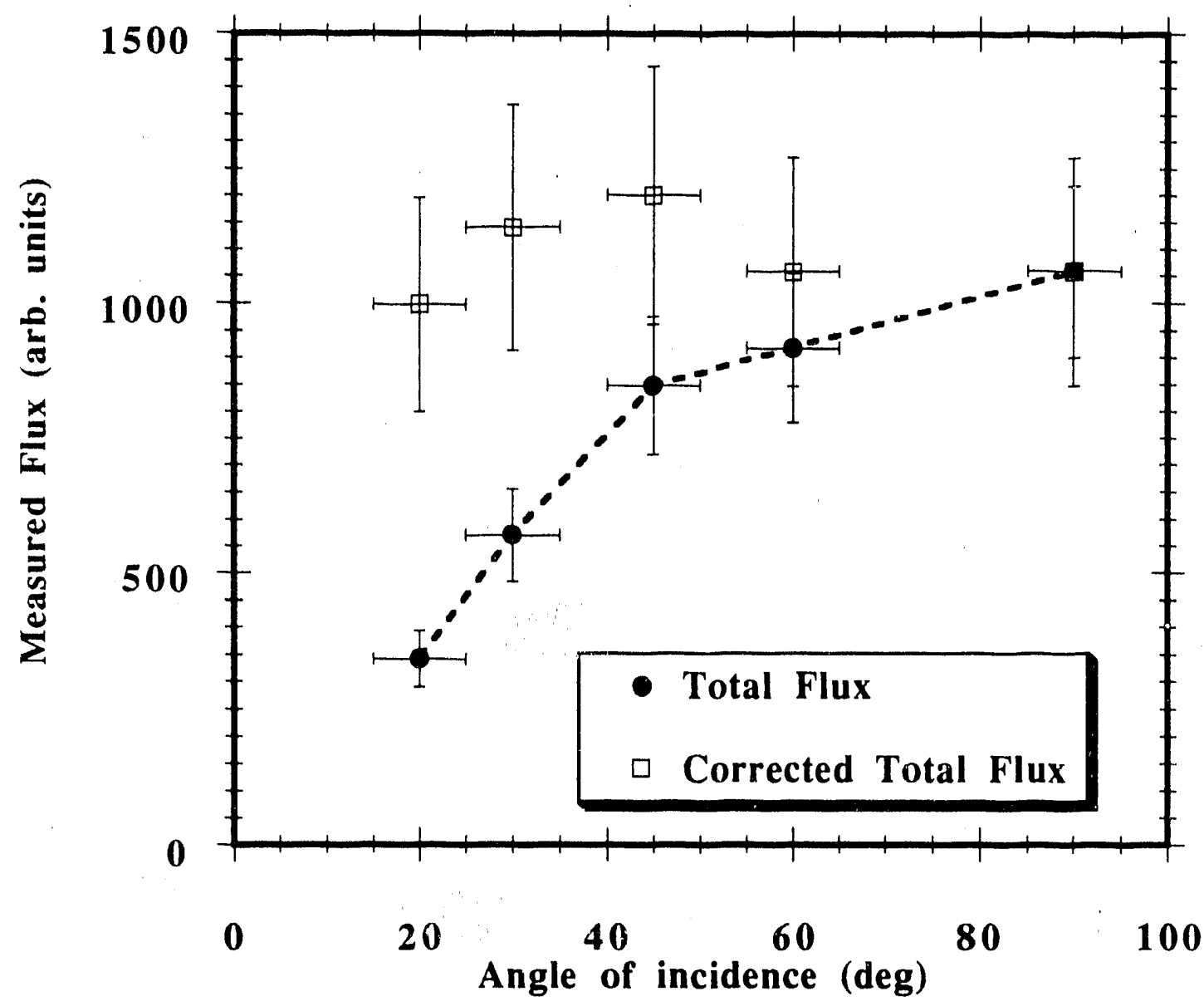

Figure 3.20: Angle of incidence dependence of light emissivity to $4.5 \mathrm{MeV}$ alphas for $\mathrm{ZnS}(\mathrm{Ag})$. 


\begin{tabular}{|l||c|c|c|c||}
\hline & detector \#6 & detector \#9 & detector \#11 & midplane det. \\
\hline poloidal angle $(\theta)$ & $-90^{\circ}$ & $-60^{\circ}$ & $-45^{\circ}$ & $-20^{\circ}$ (wall) \\
\hline relative efficiency & 1 & $0.5 \pm 0.1$ & $0.64 \pm 0.08$ & $0.25 \pm 0.04$ \\
\hline
\end{tabular}

Table 3.1: Relative optical efficiency of the four detectors. The "bottom" detector $\left(\theta=-90^{\circ}\right)$ is taken as the reference.

scale, $10 \mathrm{msec}$ of integrating time), we have for the midplane detector $6.6 \times 10^{7} \pm$ $80 \%$ (tritons +protons) $/ \mathrm{cm}^{2} / \mathrm{sec}$. We will use this number in Sect. 4.3 .4 when we will compare the experimental results with orbit code calculations. However, it is important to stress that the calibration of the detectors is still not precise enough for full, quantitative comparisons of the data with the theory. Additional measurements are under progress to improve the reliability of the absolute calibration.

By moving the same light source to the different detectors (using the same light level) it was also possible to cross-calibrate them and thus obtain a relative poloidal distribution of escaping flux. The uncertainties on that cross-calibration are much smaller than the absolute calibration which relies on a lot of factors. The results are shown in Table 3.1 for the different detectors and will be used in Section 4.3.3.

We investigated the optical resolution of the detector by using different images at the scintillator location. In Fig. 3.21 is shown the pitch angle distribution for a thin slit $\left(\sim 0.5 \mathrm{~mm}\right.$ wide corresponding to $\left.\sim 2^{\circ}\right)$ which has been lighted from the back. We can observe the effects of the optical broadening which can be fitted by a Gaussian with a FWHM of $5^{\circ}$. The overall pitch angle resolution is thus of the order of $7^{\circ}$ (FWHM). We also show in Fig. 3.22 a picture taken on the bench illustrating both the optical broadening and the vignetting effects near the sides of the scintillator. The vignetting effect could also be examined with the uniform light source and can be seen on Fig. 3.23 for the pitch angle and gyroradius distributions. Other images were also used for checking the orientation and position of the scintillator.

\subsubsection{Supplementary diagnostics}

In the study of CFP single-particle confinement we need some information about the plasma itself. The first important quantity is naturally the CFP source strength; as we saw in Chapter 1 for each neutron produced there is one $1 \mathrm{MeV}$ triton, one 3 


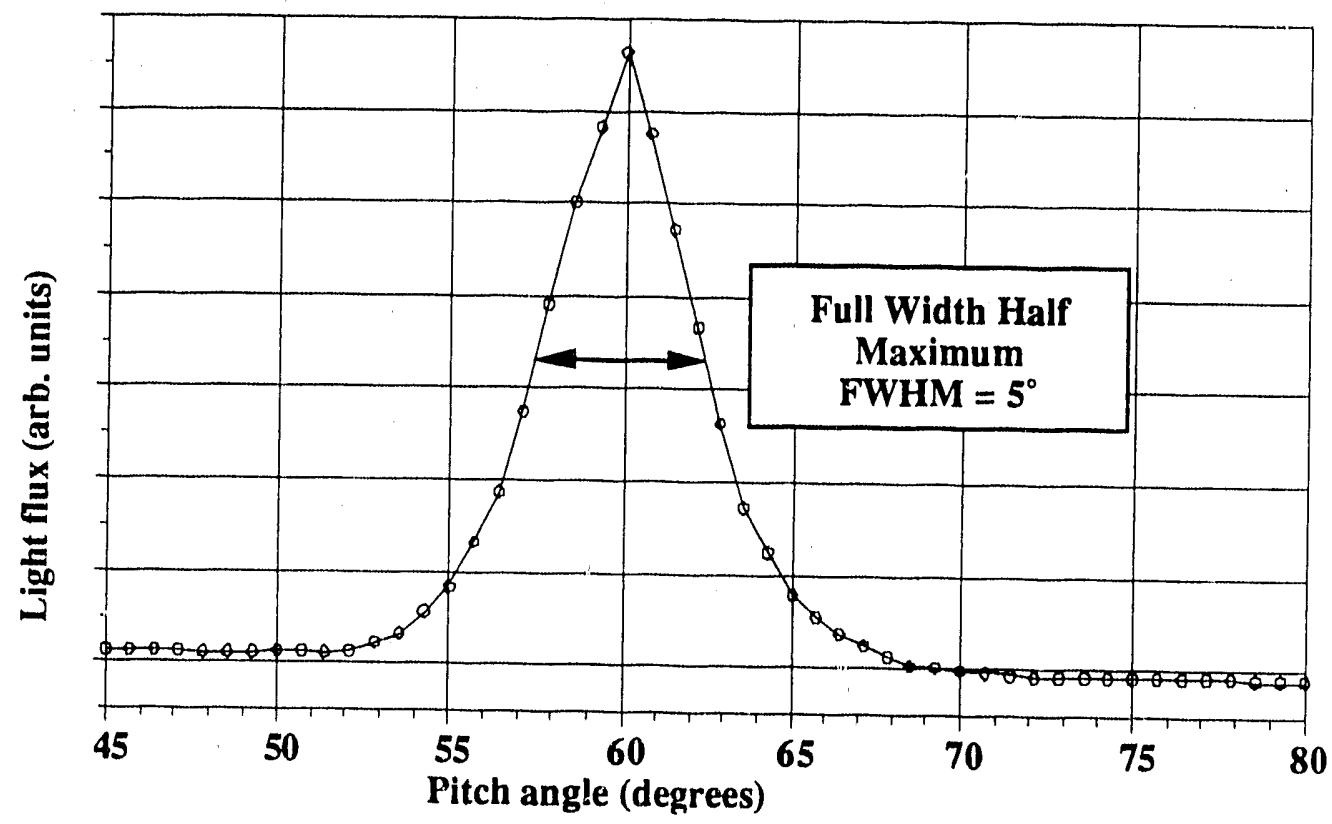

Figure 3.21: Test image (thin slit, $\lesssim 2^{\circ}$ wide) showing the optcal broadening due to the various optical components (lenses, fiber optics, camera). The resulting distribution is close to a Gaussian with a HWFM of $5^{\circ}$. 


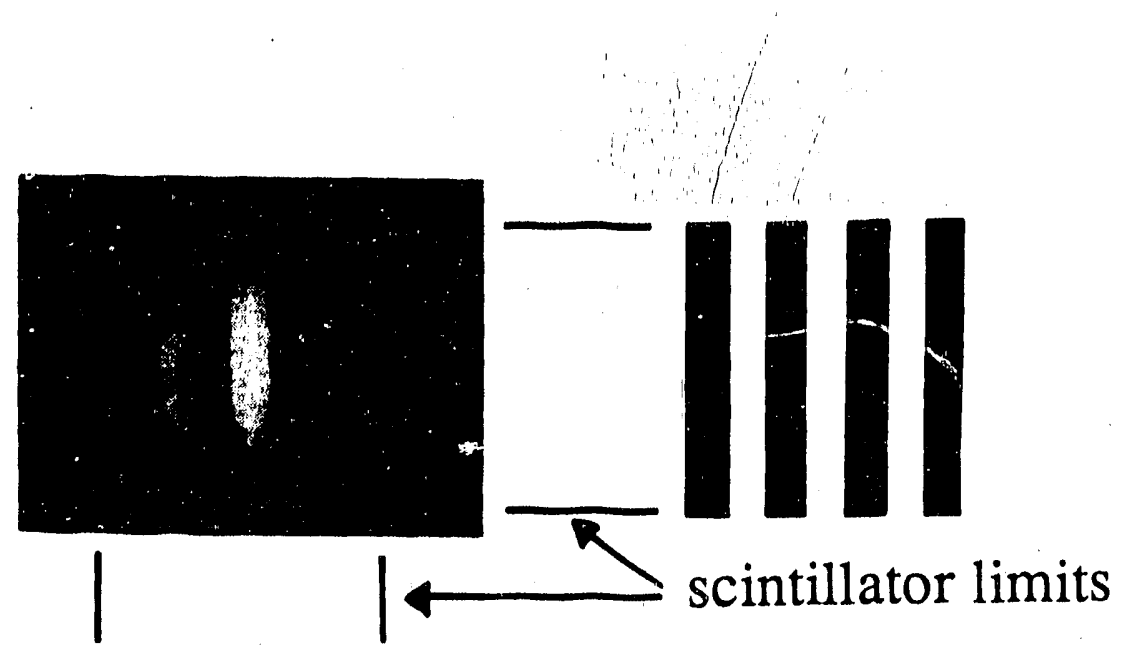

Figure 3.22: Photographic picture of the optical broadening and vignetting effects taken with the midplane probe on the bench.

$\mathrm{MeV}$ proton and one $0.8 \mathrm{MeV}^{3} \mathrm{He}$. Measurements of the neutron emission are done by using different techniques, either for global production ${ }^{112}$ or for profile measurement by using the neutron multichannel collimator. ${ }^{113}$

The other important factor is the magnetic field structure, especially the poloidal field. For our experiments we have no measurements of the current distribution and so we relied on numerical calculations made by TRANSP ${ }^{114}$ (with time dependence calculations) and SNAP ${ }^{115}$ (steady-state equilibrium) to obtain some reasonable approximations. In many of our calculations the peakedness of the current distribution was treated as a free parameter and as such represents one of the largest uncertainties.

Thermalization rates necessitate also information about electron temperature and density which can be obtained by various techniques. Magnetics data is also essential for plasma current and plasma position measurements. Finally in order to monitor the MHD activity (including sawteeth) we used the set of Mirnov coils ${ }^{116}$ and soft $\mathrm{X}$-rays ${ }^{117}$ imaging arrays. 

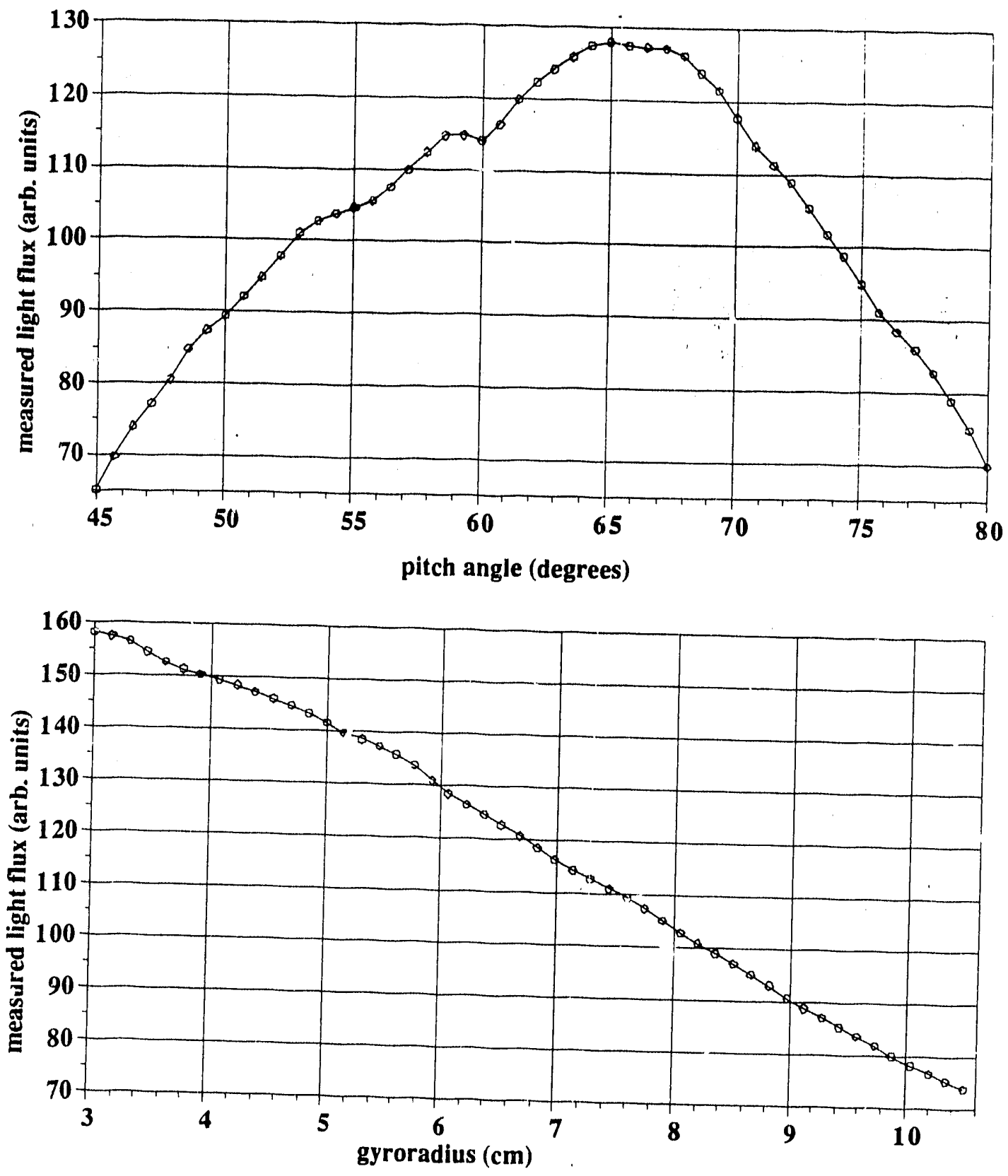

Figure 3.23: Recorded light intensity from an uniform light box illustrating the vignetting effect. On top the pitch angle distribution where the efficiency drops to $\sim 50 \%$ near the edges and at the bottom the gyroradius distribution. 


\section{Chapter 4}

\section{Characterization of CFP losses near the outer midplane}

The identification of loss mechanisms is certainly the first step in the study of confinement of CFPs. The problem is somewhat complicated by the constant presence of the first-orbit loss mechanism from which anomalous losses (like the TF stochastic ripple diffusion) must be distinguished. Calculations of first-orbit losses is a relatively straightforward problem which can be solved numerically very rapidly with an orbit code (e.g. ORBIT ${ }^{101}$ ). The problem is certainly not as straightforward when it is necessary to include mechanisms that would bring particles to the wall in a few hundred to a few thousand transit times, as in TF ripple diffusion. Because the number of detectors is limited, calculations need to be made for comparisons with a local measurement, in addition to the more common study of global confinement. Details of the numerical calculations, applied to TFTR conditions, will be presented in Sect. 4.1. From those theoretical considerations we will then move, in Sect. 4.2, to our experimental approach. In Sect. 4.3, the theoretical expectations will be compared with experimental results obtained with the midplane probe. In the final section (Sect. 4.4), we will present some additional results that could eventually be used for the study of CFP confinement.

Generally, the window of parameters in which experiments can be performed is limited, both by the operational limits of the machine and the detector, and by the requirements of the experiment. In our case, the fundarnental requirement concerns the physical mechanism itself: are TF stochastic ripple losses expected to be sufficiently large to be detectable? What conditions do we need? For example, taking 
the expression for the stochastic threshold (see Eq. 2.8) what are the parameters that can be changed for studying the ripple losses? Finally, one needs to include the operational limits.

First of all, the range of gyroradii that can penetrate in the detector is limited (see 3.1.1); consequently, for the detection of CFPs near their birth energy, the toroidal magnetic field must be kept above $\sim 3$ Tesla (TFTR maximum field strength is 5.1 $T$ at the center). To avoid disruptions-internal probes and disruptions do not live together very well - the value of $q_{c y l}$ at the edge was kept above 3.2 at all times. The choice for beam power level was a more complex problem. On one hand, because most of the CFPs originate from beam-target $(\sim 50 \%)$ or beam-beam $(\sim 30 \%)$ reactions we needed a fair amount of beam power. On the other hand, we nceded to avoid significant levels of MHD activity (however, sawteeth were most of the time inevitable) and large Shafranov shifts (orbit modeling is considerably easier with concentric flux surfaces). Typically, we found that when the detector was positioned inside the radius of the RF limiters, a minimum of $1.0 \times 10^{14}$ fusion events per second was necessary for a good signal, and when outside the RF limiters, approximately 10 times more. Finally, because of the limited heat handling capability of the probe, very large major radius plasmas could not be used efficiently (somewhat preventing complete scans of the TF ripple strength across the vacuum vessel, see Fig. 2.8). Fortunately, it was found theoretically that those restrictions were not excessive and that different confinement regimes (i.e. first-orbit or ripple loss dominated) could be studied. The range of experiments will be described in Section 4.2, and the experimental results, along with the numerical simulations, will be reported in Section 4.3.

\subsection{Theoretical expectations}

Losses associated with TF stochastic ripple diffusion involve a more gradual process than the prompt first-orbit loss process. The difference can be seen on Fig. 2.10 which show's the guiding-center orbit of a $1 \mathrm{MeV}$ triton (with $I_{p}=1.4 \mathrm{MA}, B_{T}=4 \mathrm{~T}$ and $R_{o}=2.60 \mathrm{~m}$ ) which diffuses and eventually impacts on the wall below the outer midplane. The bounce point of the $1 \mathrm{MeV}$ triton (equivalently for the $3 \mathrm{MeV}$ proton because of their identical orbits) walks down (and up) randomly, conserving $\mu$ (motion is then purely vertical) until the outer portion of the orbit hits the wall.

Before getting into detailed numerical results, it would be appropriate to use our 
diagram of Sect. 2.1.1 shown in Fig. 2.3 to illustrate the mechanism. The presence of the detector towards the midplane can be illustrated by a trapezoidal area (see Fig. 4.1) in the trapped particle region. Particles born in that range of $r / a$ and $\mu / \mu_{o}$ will penetrate in the detector as first-orbit losses. The width of the detected area is defined by the height of the aperture of the detector and is highly exaggerated in the figure. In the upper left corner of the diagram lies a region of confined trapped particles from which some will stochastically diffuse out. That diffusion vould move particles horizontally until they crossed the confined/lost boundary, either by hitting the wall or by penetrating in the detector.

In order to identify and quantify the different loss mechanisms we must look at their respective signatures. But first, it would be appropriate to recall here the properties of the detector. The probe can measure the escaping flux vs. pitch angle, vs. time, and to a lesser extent vs. energy. With the wher detectors, it can also gives the poloidal distribution. We will study, at least partially, each one of those distributions.

On the other hand, we also need to numerically sirnulate those results with our ripple diffusion model. The standard approach is to numerically solve the CFP guiding conter equations and to look at the different distributions that we mentioned above. Solving the guiding center equations already saves a lot of time over solving the complete orbit (with gyromotion) without losing any physical insight. The trajectory is represented by an Hamiltonian ${ }^{118}{ }^{119}$ (described in Appendix A.1) which uses simple coordinates $\left(r, \theta, \phi\right.$, and $\left.v_{\|}\right)$described in Fig. 2.1. This approach has been used by many researchers throughout the world, especially for conditions involving the presence of TF ripple. $4,24,25,26,120$ In most cases the emphasis has been put on global confinement and on the wall poloidal distributions of losses. This approach, which usually includes a more detailed physical description and consequently more rigorous, lacks one very important feature for us: the pitch angle distribution at the wall. An important trade-off still exists between the accuracy of the orbit calculations and the lost particles statistics at the first wall. Naturally when one gets to the detai?; of pitch angle distributions at one specific poloidal location, this problem of statistics becomes rapidly a concern (Typical Monte-Carlo calculations are using approximately 1000 particles.). A special version of the guiding-center code was necessary in our case, which we will describe below.

As we saw in Section 2.1 .3 , the transition from an oscillatory type motion to 


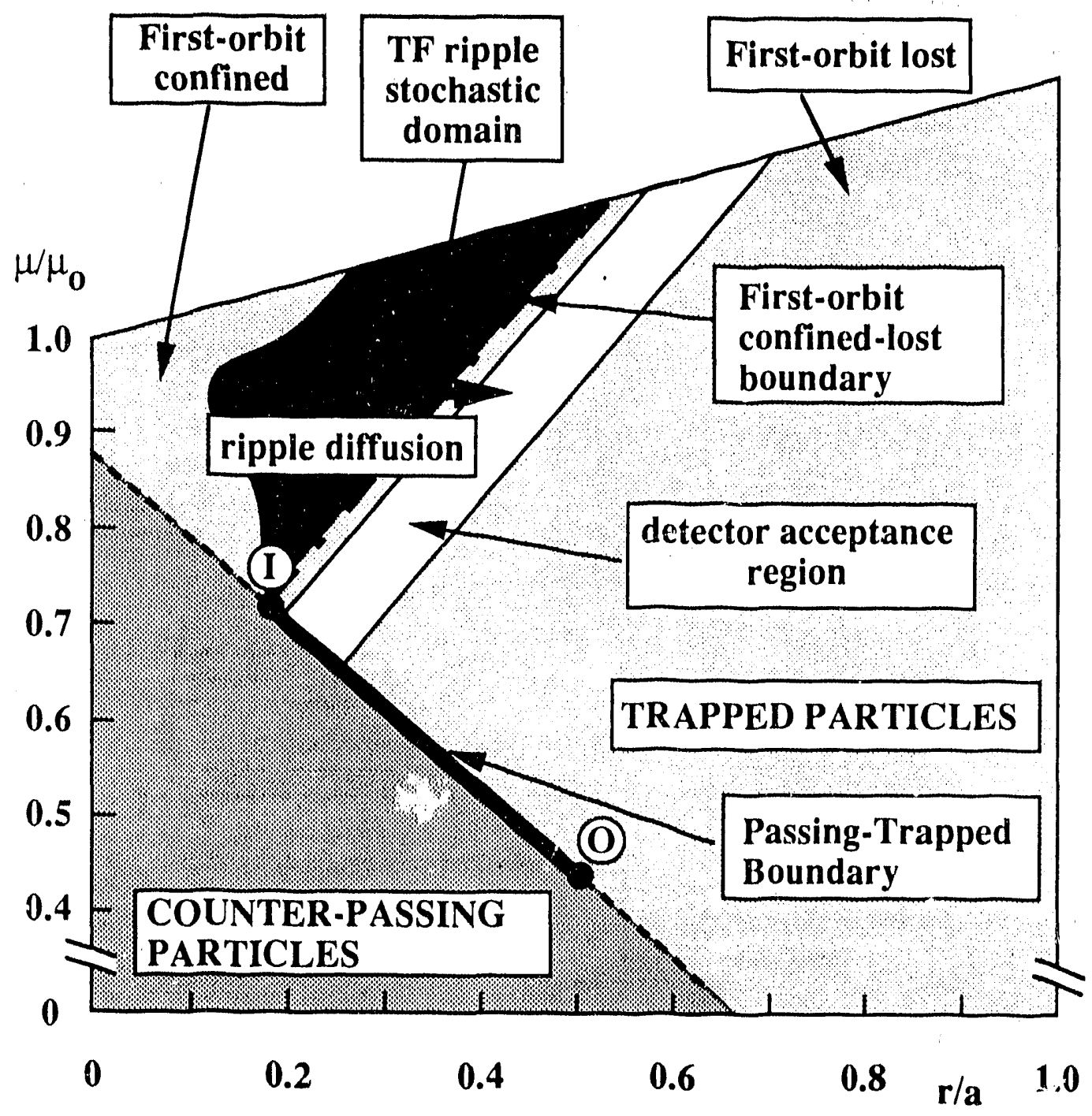

jigure 4.1: Distribution of the ripple diffusing particles in $\mu / \mu_{o}$ vs. $r /$ a space. Also indicated is the location of the probe detecting area, note that the radial extension of the probe "sight" view is highly exaggcrated. When the probe is inserted further into the vacuum vessel, its detecting area moves to the left. When the probe passes the radius of the RF limiters, it penetrates into the domain of confined CFPs (upper left corner). 
stochastic diffusion has a threshold character (see Eq. 2.8). 'This criterion for stochasticity, which can be easily computed, corresponds to a region located in the outer purtion of the plasma (see Fig. 4.2). We developed a code, MAPLOS, in order to calculate the amount of ripple losses along with their poloidal and pitch angle distributions at the wall (see also App. A.1 for details on assumed profiles, geometry and field structure). From a 3-D ( $r, \theta$ and $\chi$ ) grid birth distribution, the code starts by finding the particle's guiding-center trajectory for one poloidal transit and then retains only the trapped particles whose banana tips lie in the stochastic ripple domain. At that point, only the information about tive location of the bounce point is kept. The bounce point is then subject to ripple diffusion and we use Eq. 2.5 as the step size (where, in fact, we use the vertical component of $\Delta r$ only). The particle bounce point is then followed until the particle hits the wall, most likely just below the outer midplane.

One of the results obtained from this code is shown in Fig. 4.3 illustrating the density of bounce points after one poloidal transit. The maximum is found at a minor radius of approximately $a / 3$ around $\theta \sim 2 \pi / 3$. Also shown is the location of the stochasticity threshold, indicating where ripple-lost particles originate and what fraction would be lost. From there it is easy to obtain information about the global confinement by simply counting the number of particles located in the different loss cones. Shown in Fig. 4.4 are the calculated fraction of lost particles through first-orbit and ripple versus the plasma current. For both the $R_{o}=2.60 \mathrm{~m}$ and the $R_{o}=2.45 \mathrm{~m}$, first-orbit losses decrease exponentially with current, whereas ripple losses would peak around 1.4 MA. With increasing plasma current, the ripple losses first increase, because the first-orbit confinement of CFPs improves (at low current, all trapped particles are first-orbit lost), and then decrease, since the extent of the stochastic domain shrinks, explaining the peaking in ripple losses. Similar calculations, involving global confinement only, have also been performed by White ${ }^{28}$ by using the code RIPLOS but which does not keep track of the particle's impact on the wall.

The results obtained with MAPLOS were compared with the ones obtained with Tani's OFMC code which calculates total losses in a non-axisymmetric field using a guiding-center integration technique and which includes a complete description of the fields and collisions. The codes were found to be in very good agreement ${ }^{125}$ over the current dependence and poloidal distribution of $3.5 \mathrm{MeV}$ alphas losses in TFTR. 


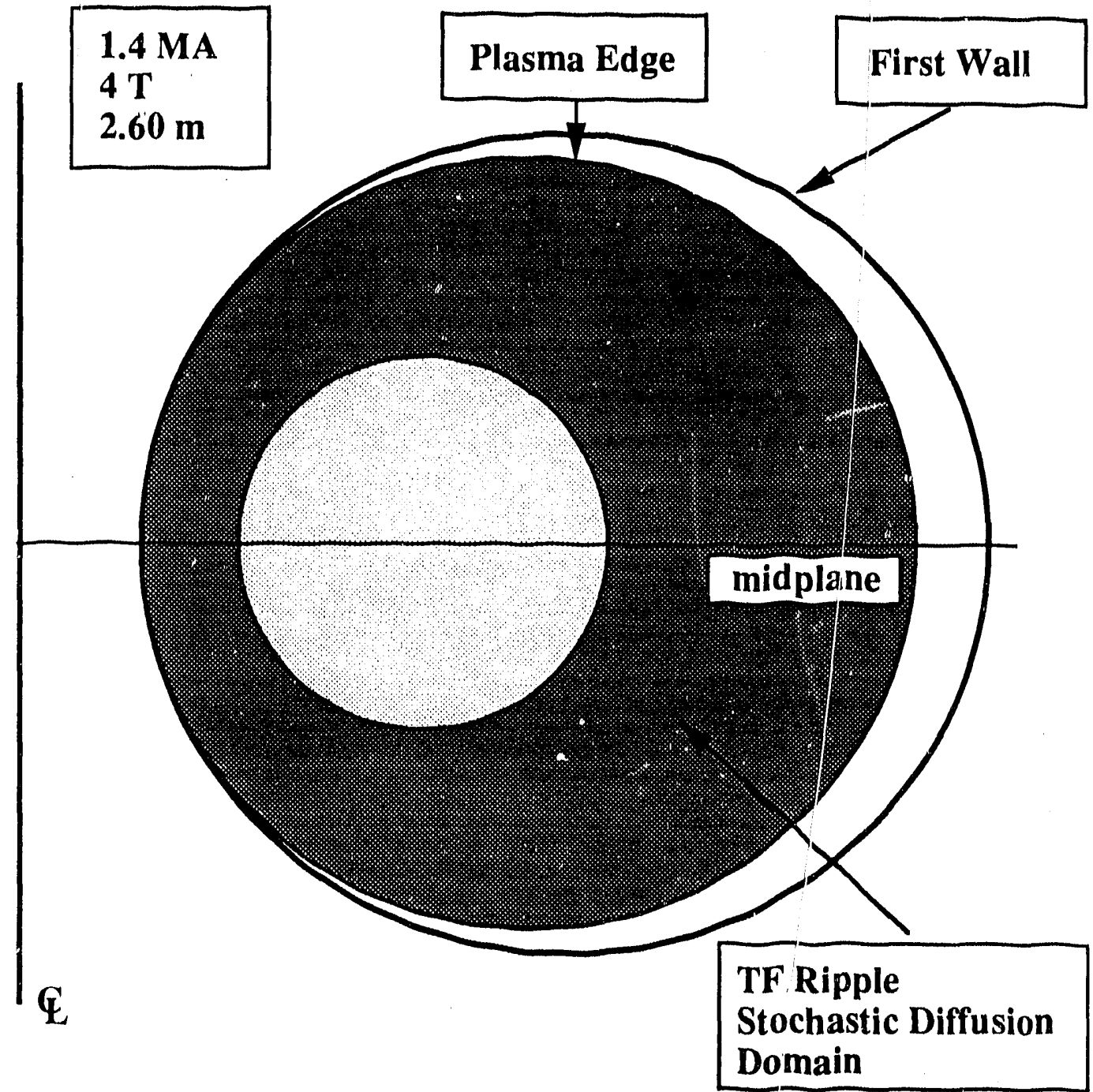

Figure 4.2: Ripple stochastic domain for TFTR at $I_{p}=1.4 \mathrm{MA}, R_{o}=2.60 \mathrm{~m}$ and $B_{T}=4 T$. 


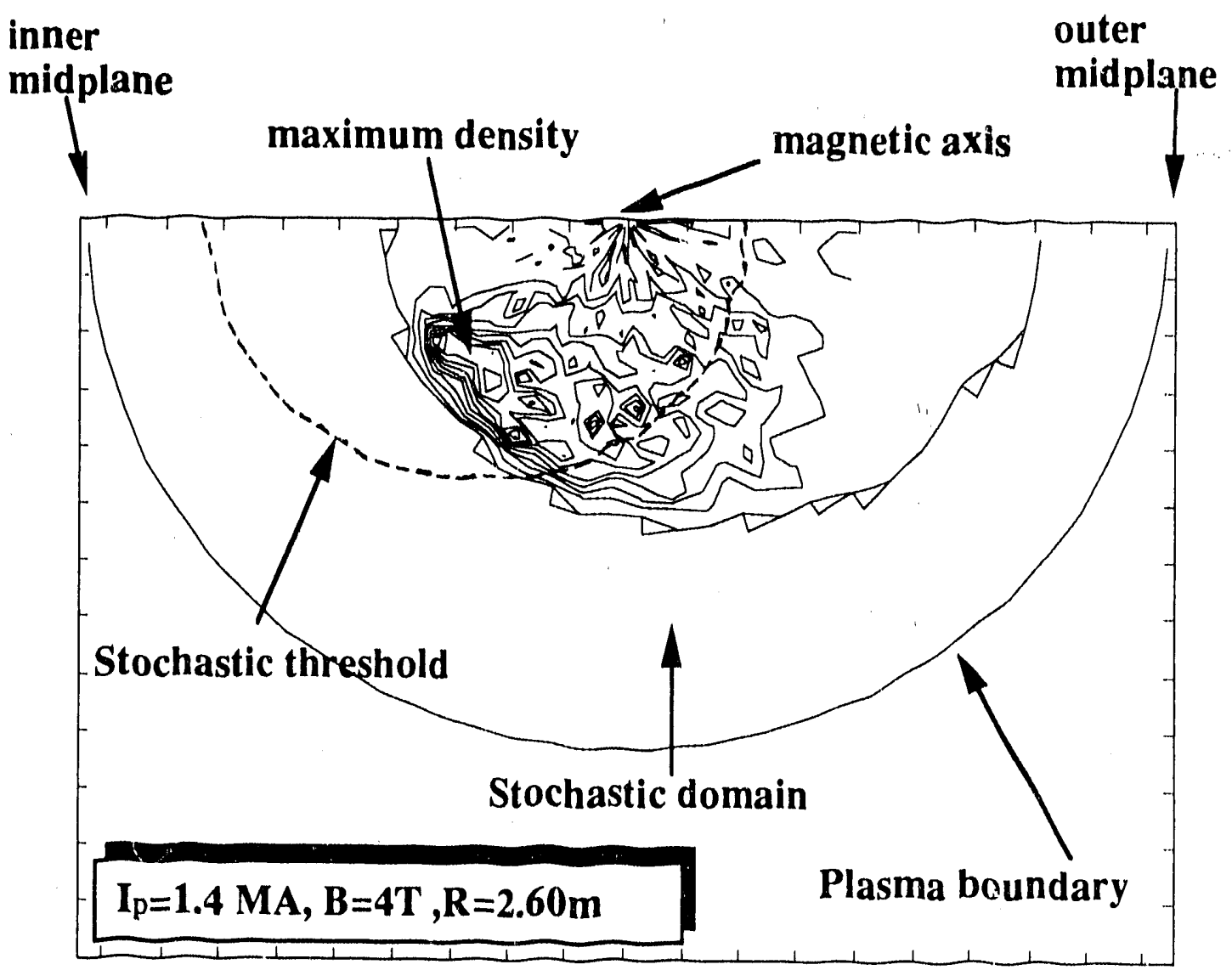

Figure 4.3: Poloidal density of bounce points for first-orbit confined trapped particles $\left(I_{p}=1.4 M A, B_{T}=4 T\right.$ and $\left.R_{o}=2.60 \mathrm{~m}\right)$. 

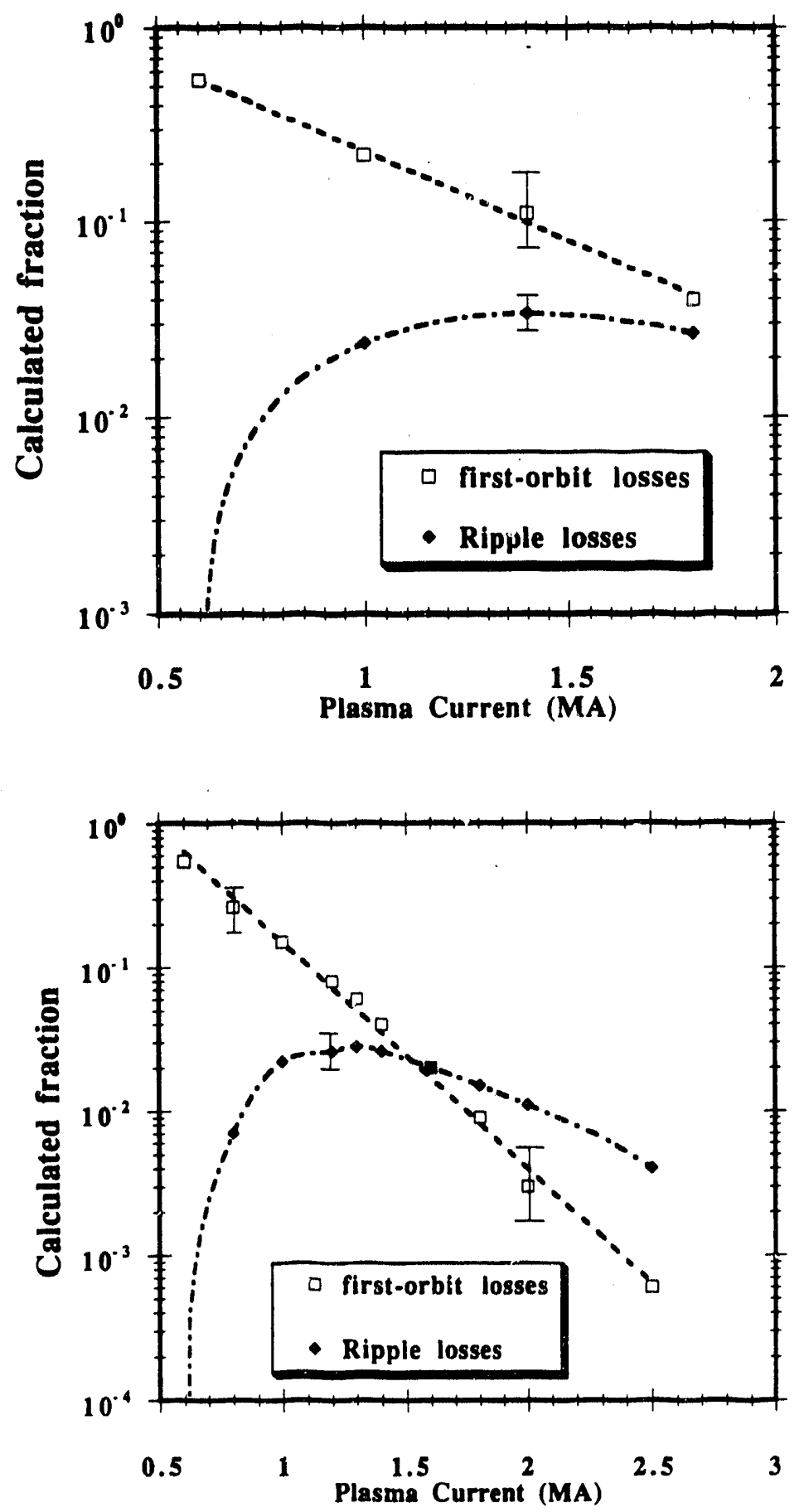

Figure 4.4: Calculated total loss fraction versus plasma current for tritons (and protons) and for two different major radii (on top $R_{o}=2.60 \mathrm{~m}, B_{T}=4 \mathrm{~T}$, and at the bottom $R_{o}=2.45 \mathrm{~m}, B_{T}=5 \mathrm{~T}$ ). 
The next step, which is of crucial importance for our measurement and for the design of future machines, is to calculate the different distributions at the wall. The first aspect is naturally the distribution of their impact location. As the CFPs diffuse with a step size of the order of a centimeter, particles that miss the wall on one pass would impact on the wall very close to the outer midplane. That distribution of wall impacts is a strong function of the step size (i.e. Eq. 2.5), but also of the banana width. Particles at high current (which have a smaller banana width) tend to be within a step size of the first wall on a much longer poloidal distance and so are likely to be more evenly distributed poloidally. Details of the first wall geometry will be crucial in non-circular machines like BPX and ITER. ${ }^{6}$ Figure 4.6 show's a typical poloidal distribution for first-orbit and ripple losses. For first-orbit losses we found, as expected, ${ }^{121-124}$ that they are widespread on the bottom but, on the other hand, ripple losses are peaked at the midplane. ${ }^{121,125}$ This high localization of lost alpha particles is the most serious wall loading problem in large tokarnaks. 'This wall loading problem could also be amplified if, in addition to being poloidally localized, first wall asymmetries would cause toroidally localized losses.

As a first approach we took the first wall of TFTR as uniform toroidally and we assumed the aperture to be flush with the wall. This approximation cannot yield the full information needed to compare with our measurements and so we also added the presence of the RF limiters (described in more details in Ch. 5). However, this addition does not solve the problem of the actual detector location which is located between the RF limiters and the wall, and has a very small toroidal extension. Since the geometry is no longer axisymmetric, the addition of the RF limiters also removes a sizable fraction of the much needed statistics. In some of the sections we will show the numerical results with and without the presence of the RF limiters, as a comparison with the experimental results.

Another critical point related to the results obtained in Fig. 4.5 is how rapidly particles are being lost. The vertical step size being relatively large, from a fraction of millimeters to several centimeters (depending on plasma parameters), and with a bounce time of about $q R / v_{\|} \simeq 10 \mu$ s the majority of TF ripple diffusing tritons would escape in typically less than $50 \mathrm{~ms}$ (and 3 times less for the proton) which is at least 10 times smaller than their slowing down time. Shown in Fig. 4.7 is the calculated confinement time before impact on the wall. We observe a strong exponential decrease of escaping flux versus their time of impact. Since the confinement time is much 


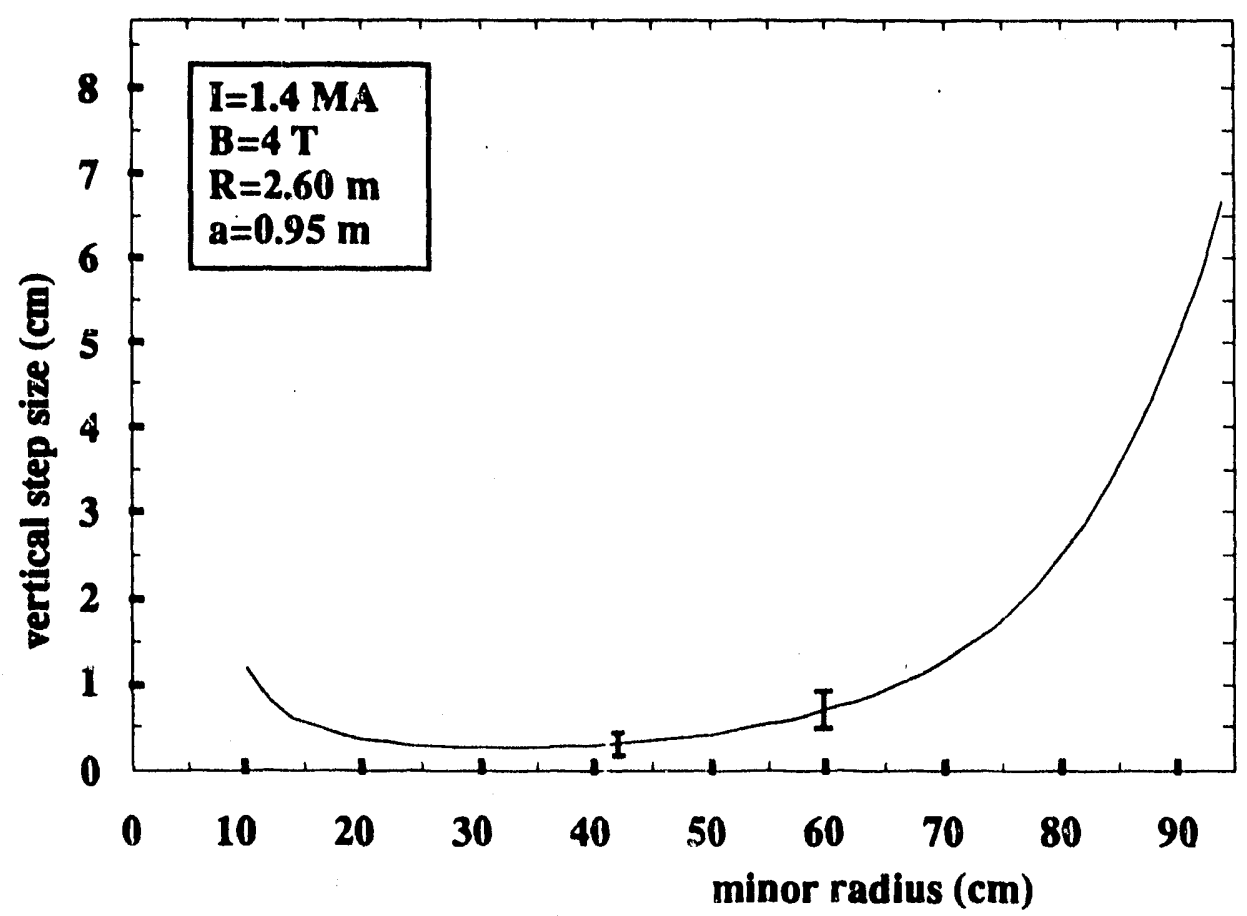

Figure 4.5: V'ertical position dependence of the ripple step size just below the plasma center $\left(\theta=90^{\circ}\right)$ for $I_{p}=1.4 \mathrm{MA}, B_{T}=4 T, R_{o}=2.60 \mathrm{~m}$. The error bars represent the variations due to different current profiles and uncertainty in the ripple amplitude.

smaller that the slowing-down time, it is legitimate to assume that the particle energy is conserved during ripple diffusion.

Finally, ine information we are especially interested in is the pitch angle distribution. As we saw previously, the pitch angle resolution of the detector is relatively good, $\pm 3^{\circ}$ in absolute angle and $\pm 7^{\circ}$ in peak resolution, so we anticipate the possibility of distinguishing between different loss mechanisms. For given detector location and plasma corditions, particles are escaping at different pitch angles each corresponding to an orbit originating from different regions of the plasma. This spatial resolution is especially important when losses have a spatial threshold character like first-orbit and TF ripple losses. We know that for first-orbit losses the pitch angle of maximum flux corresponds to the orbit passing the closest to the plasma center, the source being highly peaked there. For medium and high current shots this pitch angle corresponds to the fattest banana, at the boundary between passing and trapped orbits. In that case the bounce points of these orbits are located on the inner side of the midplane.

As we know, the TF ripple diffusion affects only trapped particles, consequently any particle lost on their first orbit would not be available for ripple diffusion. In 


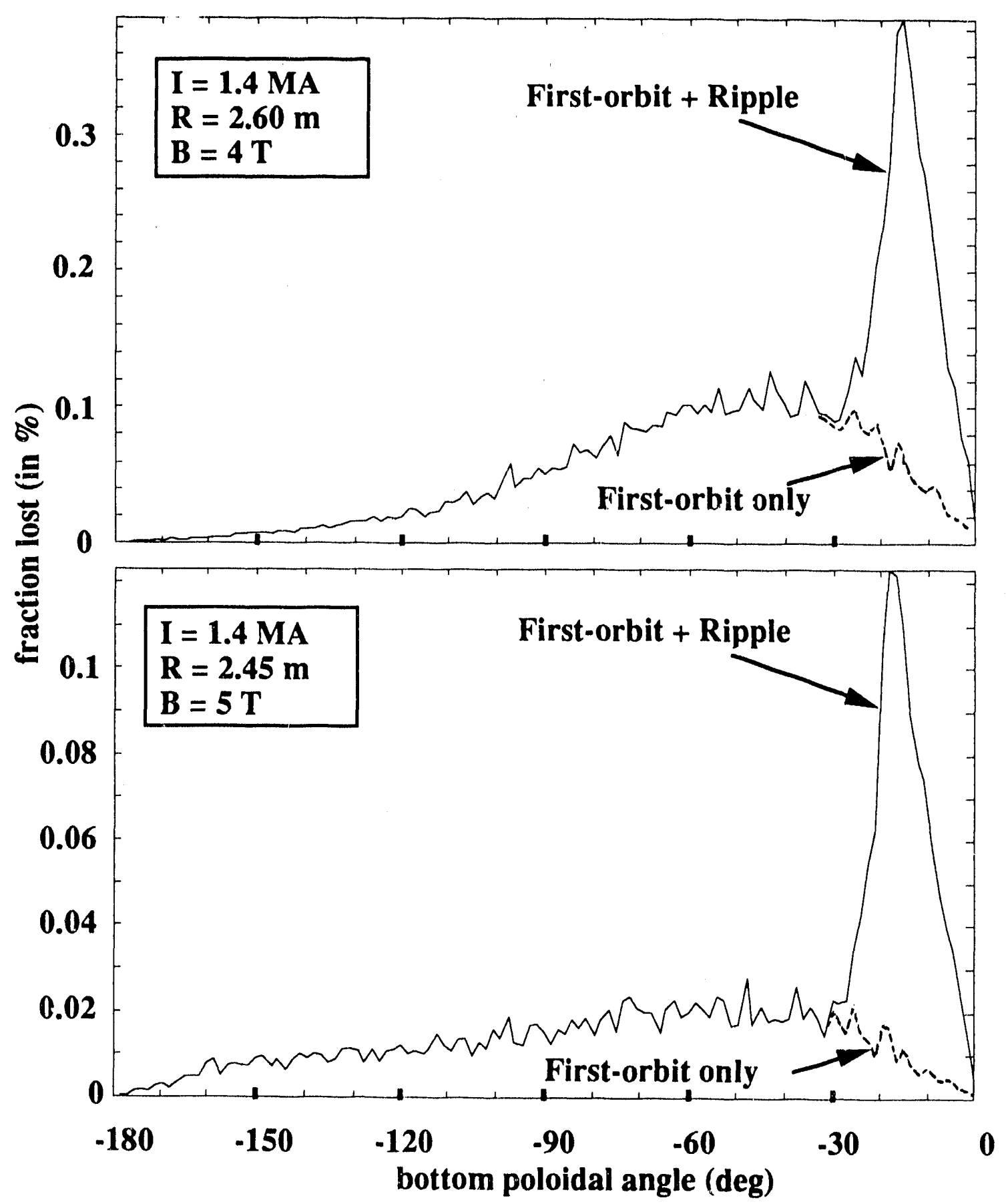

Figure 4.6: Poloidal distribution of first wall impacts due to first-orbit and TF ripple losses for $I_{p}=1.4 M A$. 


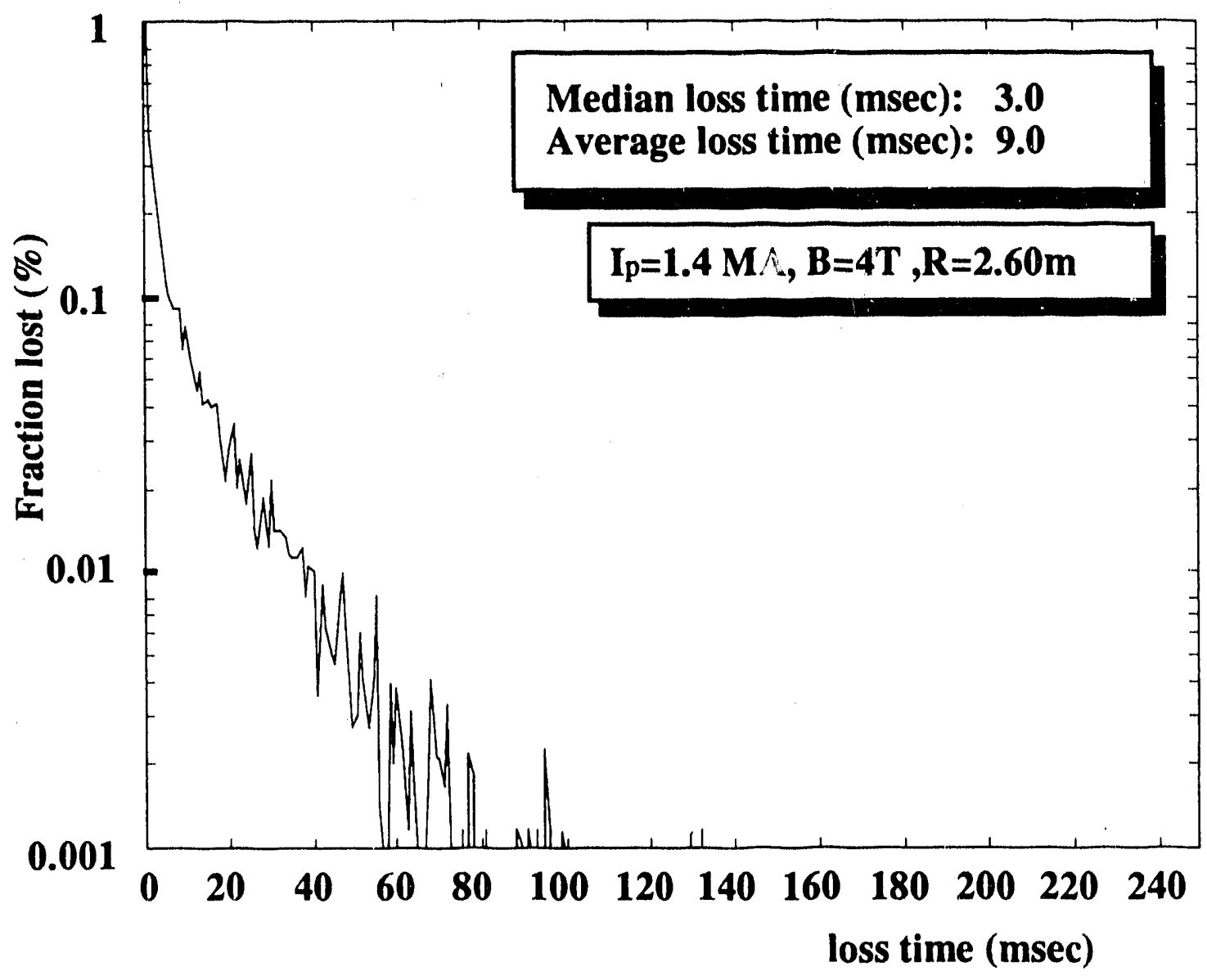

Figure 4.7: Calculated distribution of time elapsed before impact for ripple diffusing particles. Particles escape have an average loss time of 9 msec and a median of 3 msec in the case of $I_{p}=1.4 M A, B_{T}=4 T$ and $R_{o}=2.60 \mathrm{~m}$. 
Fig. 4.3 we see that the majority of first-orbit confined particles subject to ripple diffusion are located just below the magnetic axis. We show in Fig. 4.8 the orbits corresponding to peaks in first-orbit and ripple losses. By using energy and magnetic moment conservation ve can derive simply their corresponding toroidal pitch angle at the detector $\left(\chi_{\text {det }}\right)$.

$$
\mu=\frac{E \sin ^{2} \chi_{\text {det }}}{B_{\text {det }}}
$$

where

$$
B_{\text {det }}=\frac{B_{o}}{1+\frac{r_{\text {det }}}{R_{o}} \cos \left(\theta_{\text {det }}\right)}
$$

is the magnetic field at the detector $\left(R_{o}=2.65 \mathrm{~m}, r_{\text {det }} \approx 100 \mathrm{~cm}\right.$ and $\left.\theta_{\text {det }}=-20^{\circ}\right)$. Since the bounce point of the first-orbit peak is at $\theta_{b}=\pi$ and the ripple peak at $\theta_{b} \approx \pi / 2$ and since $\mu$ and $E$ are conserved:

$$
\begin{array}{cl}
\frac{\sin ^{2} \chi_{\text {det }, f / 0}}{B_{\text {det }}}=\frac{1-\frac{r_{b}}{R_{o}}}{B_{o}} & \text { (first-orbit) } \\
\frac{\sin ^{2} \chi_{\text {det, ,ipple }}}{B_{\text {det }}}=\frac{1}{B_{o}} & \text { (ripple) }
\end{array}
$$

where $r_{b}$ is the minor radius of the bounce point. We find that $\chi_{\text {det, } f / o} \approx 55^{\circ}$ (with $r_{b} \approx 30 \mathrm{~cm}$, at $I_{p}=1.4 \mathrm{MA}$ ) and that $\chi_{\text {det,ripple }} \approx 65^{\circ}$. Note however, that contrary to the first-orbit case (where $r_{b}$ is a strong function of $I_{p}$ ) the estimate of the pitch angle for ripple losses is independent of the plasma current. The variation in the orbit of the peak pitch angle for first-orbit losses is illustrated in Fig. 4.12. We show in Fig. 4.9 an example of a pitch angle distribution at the wall (at $\theta=-20^{\circ}$, the probe approximate poloidal location) where both peaks are present and of the same order of magnitude. The angular separation is large enough for resolution and this case will be discussed further in Sect. 4.3.2, along with the experimental results. However, in the majority of cases the distribution is dominated by one process, producing only one peak. It is also important to stress that the calculated ratio of first-orbit to TF ripple losses at the detector is a function of not only the global fraction lost by either process but also a strong function of the their relative poloidal distribution on the first wall.

For the pitch angle distributions calculated at specific wall locations (i.e. at a detector poloidal location), we typically average the calculated flux over a range of 


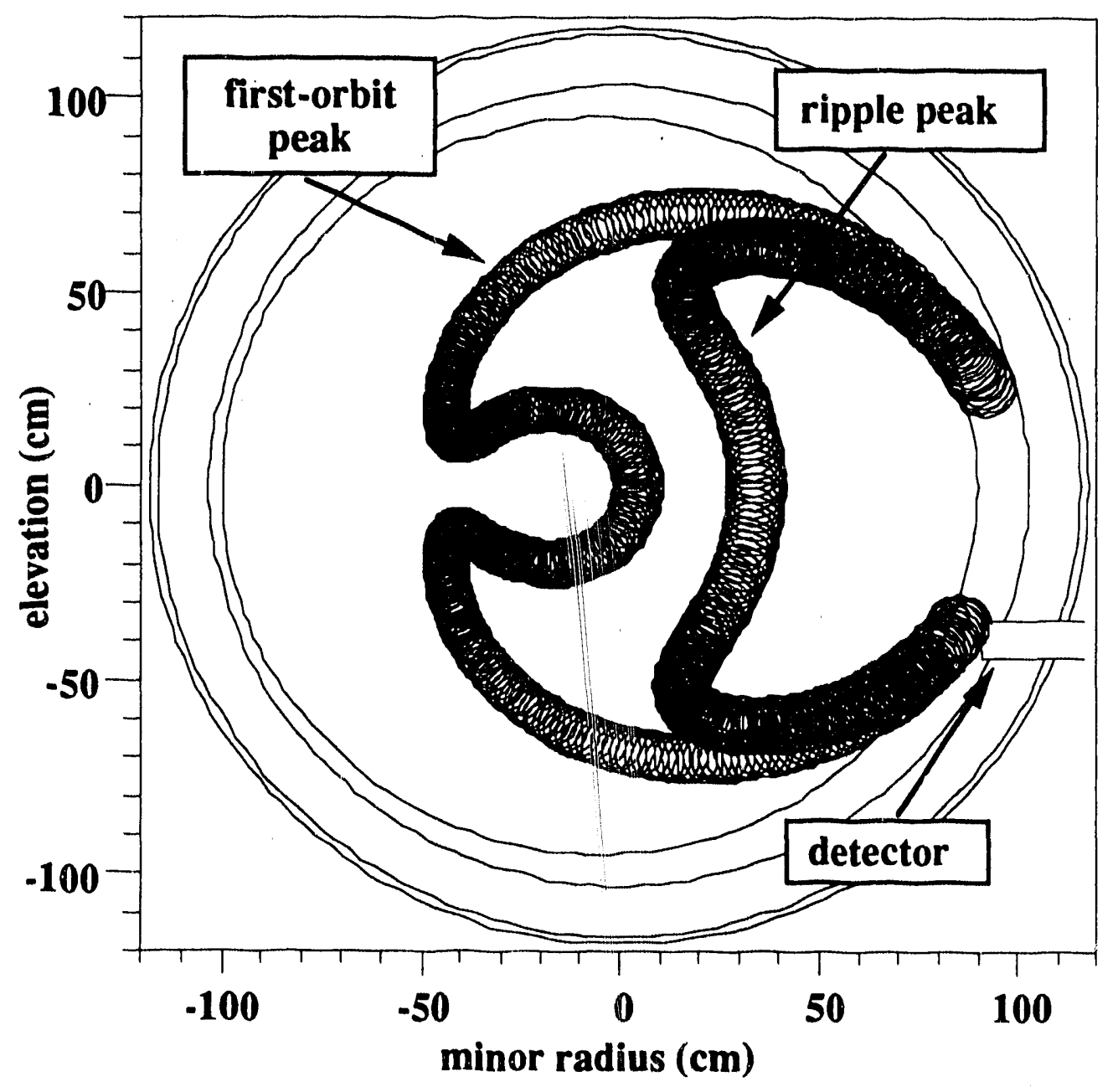

Figure 4.8: Comparison of the orbits for peaks in first-orbit and ripple losses for $I_{p}=1.4 M A, B_{o}=4 T$ and $R_{o}=2.60 \mathrm{~m}$. The pitch angle at the detector is $\approx 55^{\circ}$ for the first-orbit peak and $\approx 65^{\circ}$ for the ripple peak. 


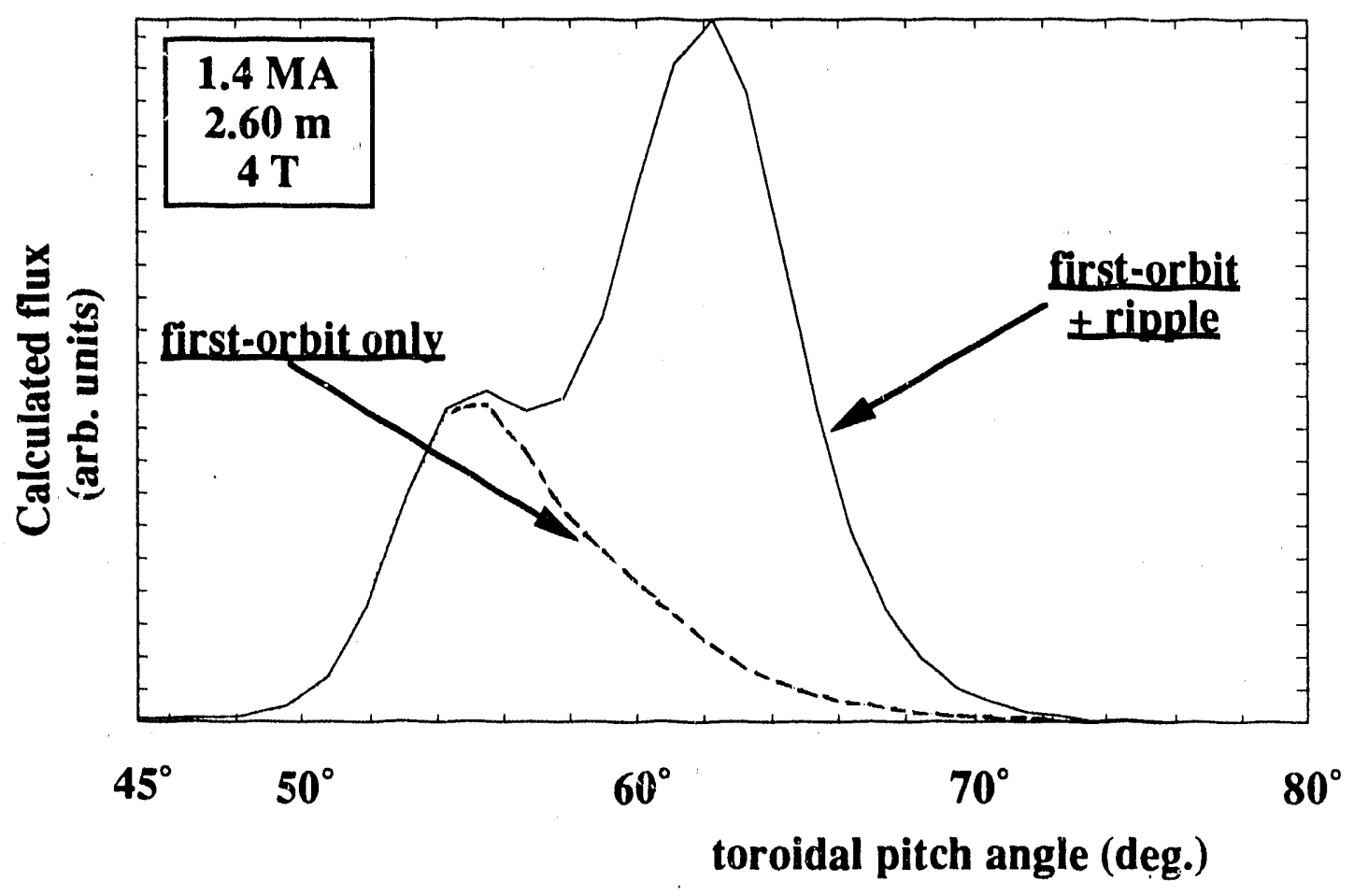

Figure 4.9: Example of a pitch angle distribution on the first wall, at the probe location below the midplane, for $I_{p}=1.4 \mathrm{MA}, B_{T}=4 \mathrm{~T}$ and $R_{o}=2.60 \mathrm{~m}$. The presence of two peaks is clearly visible here, the left peak corresponding to first-orbit losses and the right one to TF ripple losses. 
$\pm 2.5^{\circ}$ in $\theta$ (poloidal angle). The pitch angle distribution calculated by MAPLOS were also compared with ORBIT for bench-marking. We found that at low current the distributions agreed within $15 \%$ for the flux and within $\pm 1^{\circ}$ for the peak in the distribution. At higher current, some shift $\left(\lesssim 3^{\circ}\right)$ has been observed. We believed that this shift is due to the simple expression used in MAPLOS for the magnetic field where we took the toroidal field component only. However, the shape of the curve is not affected, it is simply shifted slightly.

\subsection{Description of the experiments}

Considering the relative variability of tokamak operations and the limited number of shots, care must be taken to choose the most appropriate approach to the study of TF ripple losses. By looking at Eq. 2.8, and by identifying the fundamental variables, i.e. the plasma current $I_{p}$, the toroidal field $B_{T}$ and the aspect ratio $\epsilon=a / R_{o}$ we see that:

$$
\begin{array}{r}
\delta_{s} \propto \epsilon^{-\frac{1}{2}} I_{P}^{\frac{5}{2}} B_{T}^{-\frac{3}{2}} \\
\delta \propto \exp (\epsilon)
\end{array}
$$

where $\delta_{\mathrm{s}}$ is the stochastic threshold (Eq. 2.8) and $\delta$ is the ripple amplitude (Eq. 2.3). Basically this gives us three main avenues for the study of ripple losses, one for each variable.

A scan in toroidal field strength would have a great advantage. In the small banana width approximation ${ }^{8}$ (which is a poor assumption for CFPs in low plasma current discharges)

$$
\Delta_{b} \approx \frac{2 q \rho}{\sqrt{\epsilon}}
$$

which is independent of the toroidal field strength. Thus it would be theoretically possible to vary the number of ripple lost particles without varying the amount of firstorbit losses by simply varying the toroidal field strength. Unfortunately, as mentioned at the beginning of the chapter, the range of possible field strengths is limited to 3 to 5 Tesla representing only a small difference (a few centimeters in minor radius) in the stochastic loss domain (especially because the ripple has an exponential radial 
dependence). This scan would also be complicated by the presence of large scale MHO activity at low $B_{T}$ and/or by the changes in current profile.

Potentially, changing the plasma aspect ratio can also help in identifying the loss mechanisms. In this case, we can vary both the threshold and the ripple strength. Complications arise though, when the plasma is brought too close to the probe head which has limited heat handling capabilities. The amount of first-orbit losses is also affected because of the variable location of the walls/limiters with respect to the plasma. Nevertheless, it is possible to compare the confinement for different aspect ratios and we will indirectly compare cases made with various plasma major radii.

Finally, the last possible variation is over the plasma current. In this case, it can be varied widely, e.g. from 0.6 to $2.5 \mathrm{MA}$ (plasma stability being the only major operative difficulty). The main attraction really comes from the different confinement regimes (very large to very small losses, dominated by either first-orbit or ripple losses) that can be reached. An increase in plasma current would decrease the extension of the stochastic domin but also reduce the particle's banana width and would keep them further away from loss cones. For example, numerical calculations show that, at low current, losses can exceed $60 \%(\leqslant 0.6 \mathrm{MA})$ whereas at high current they would be small, a few percent ( $Z 1.6 \mathrm{MA}$ ) as shown in Fig. 4.4. Most of the experimental results were obtained during plasma current scans and will presented in detail in Section 4.3.

In addition, some preliminary results will be presented on current ramps, beam power dependence and small compression obtained by piggy-backing other experiments. Finally, experiments using the motion capability of the probe will be described in Chapter 5.

\subsection{Plasma current dependence}

As mentioned in the previous section, the most basic, and in many ways, the most attractive experiment consisted of varying the plasma current. As one can see from Fig. 4.4 the amount of first-orbit losses decrease drarnatically with current. This strong dependence on plasma current is mainly caused by a reduct ' $n$ of the banana width. TF ripple losses are somewhat more complex. At low current, although the stochastic region covers the whole plasma (e.g. at $0.6 \mathrm{MA}$ ), the relevant trapped CFPs are already lost on their first orbit. The effect of increasing plasma current 
is twofold, the stochastic region recedes towards the plasma edge (as expected from Eq. 2.8) and in the meantime, the orbits stay closer to the center, out of the stochastic region.

We did the experiment with two different conditions, one with $R_{o}=2.60 \mathrm{~m}, B_{T}=4 \mathrm{~T}$ and one with $R_{\mathrm{o}}=2.45 \mathrm{~m}, B_{T}=5 \mathrm{~T}$ where the ratio of first-orbit to TF ripple losses would be slightly different (see Fig. 4.4). However, the emphasis will be put on the former case which indicates the distinctive presence of two loss mechanisms at the same time. Neutral beam power was kept at the minimum necessary for a good signal, in order to avoid MHD activity, which was absent in all cases except for sawteeth activity, present at higher currents.

\subsubsection{Current dependence of the escaping flux}

The first thing we wish to look at is the plasma current dependence of the total escaping flux as measured by the midplane probe. With the detectors we obtain only a very local measurement of the confinement and we cannot directly compare with global calculations presented in Fig. 4.4. In Fig. 4.10 are shown the current dependences of the total flux measured at the detector (light signal is integrated over all pitch angles and gyroradii). In the large plasma case, we noticed a very large increase of escaping CFPs at 1.4 MA. In addition we plotted the numerical simulations obtained from MAPLOS using the measured source profile (by the neutron multichannel collimator ${ }^{113}$ ) and the calculated current profile (by the steady-state code SNAP ${ }^{115}$ ). In both cases the curves are normalized at the lowest current point where the losses should be largely dominated by first-orbit losses. In the $R_{o}=2.45 \mathrm{~m}$ case the local maximum occurs at $0.8 \mathrm{MA}$, a lower current than the $R_{o}=2.60 \mathrm{~m}$ case. The sudden increase is due to a big change in orbit topology; practically no banana orbits are found at 0.6 MA (with very large first-orbit losses), whereas at 0.8 MA the first-orbit confinement is considerably larger. At 0.8 MA many particles now firstorbit confined (from the $0.6 \mathrm{MA}$ case) are instead ripple lost. This quick change in first-orbit confinement is visible in Fig. 4.4 where the calculated fraction of first-orbit losses drop faster with current in the $R_{o}=2.45 \mathrm{~m}$ case than in the $R_{o}=2.60 \mathrm{~m}$ case.

A certain difference between the two cases is noticeable. In the case of a "standard" plasma $\left(R_{o}=2.45 \mathrm{~m}\right)$ the transition of loss mechanisms from first-orbit to TF stochastic ripple diffusion occurs at lower plasma current, an expected behavior from 

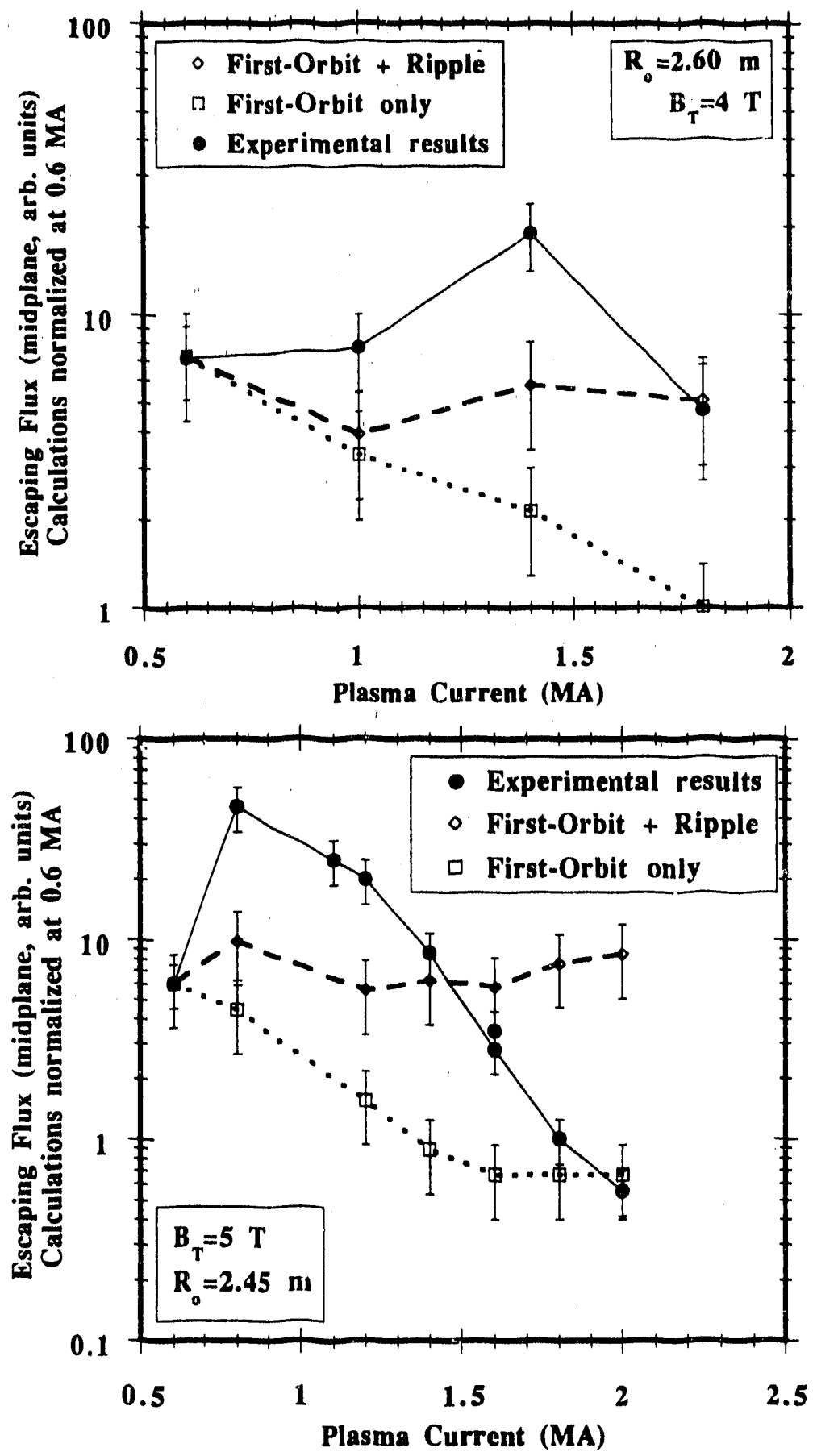

Figure 4.10: Escaping flux versus plasma current as measured by the midplane detector for two different plasma conditions (top: $R_{o}=2.60 \mathrm{~m}$ and $B_{T}=4 \mathrm{~T}$; bottom: $R_{o}=2.45 \mathrm{~m}$ and $B_{T}=5 \mathrm{~T}$ ). Long dash lines correspond to calculations including first-orbit and ripple losses whereas small dash lines corresponds to first-orbit losses only. Curves are all normalized at $0.6 \mathrm{MA}$. 
Fig. 4.4. In both cases, the peak in losses is totally unexpected from first-orbit considerations only. Calculations were able to predict a local maximum from TF ripple losses, for both $R_{o}=2.45 \mathrm{~m}$ and $R_{o}=2.60 \mathrm{~m}$ cases, although the experimental results could not be reproduced completely. The reasons for that discrepancy could come from many factors. One of those reasons refers naturally to the extent of our knowledge of the current and source profiles. A global uncertainty of maximum $\sim 50 \%$ (depending on current) is found when considering in the numerical calculations, possible variations in the current, source and ripple profiles. Another big factor originates from the wall and detector geometries. In the calculations we actually compute the flux to the first wall, not to the detector itself, and we do not include the irregularities of the wall such as the RF liniters. Since the detector's aperture is located behind the radius of the RF limiters, we expect some of the flux to be blocked by the limiters before it can reach the aperture. The fiux would be increasingly reduced with higher current since the diffusion step size decreases with current (see Eq. 2.5). The next chapter describes in more details these effects. In addition to these effects we also neglected the fact that the aperture is actually not at the wall but located between the wall and the RF limiters where some of the detected particles may not be their last orbit to the wall.

\subsubsection{Pitch angle distribution}

The ability to resolve the pitch angle distribution can be extremely useful in identifying the spatial origin of lost particles and the physical mechanism of diffusion. First-orbit distributions can be calculated relatively easily for a specific wall location (i.e. at one detector) by using, for example ORBIT. The task is eased mainly because of the short trajectory involved. However, in the case of TF stochastic ripple losses, this can be obtained only at the cost of long numerical calculations that we described in Sect. 4.1 .

We found from both numerical calculations and experimental results that, in most cases, the distributions are dominated by one loss process or another. Rare were the cases where both would be present at the same time in roughly the same amount. The losses also need to be sufficiently separated in pitch angles to allow resolution of the different contributions. Fortunately, we do not need to have both processes to be always visible since TF ripple losses are expected to occur at a significantly different 
pitch angle than first-orbit losses. As we saw in Sect. 4.1 we expeci more deeply trapped particles to be ripple diffusing, thus appearing at an higher pitch angle on the scintillator.

The most illustrative cases were obtained during the current scan at large major radius $\left(R_{o}=2.60 \mathrm{~m}\right)$ in which the features from first-orbit and ripple losses are clearly distinguishable. Other cases will also be shown below. In Fig. 4.11 we show the measured pitch angle distributions for the four different currents $(0.6,1.0,1.4$ and 1.8 MA, at $B_{T}=4 \mathrm{~T}$ and $\left.R_{o}=2.60 \mathrm{~m}\right)$ along with the numerical simulations. Note that the calculated distributions have been smoothed (see Sect. A.1 with the measured resclution described in Sect. 3.2.2

In ali cases, the numerical simulations were able, overall, to reproduce fairly well the experimentally obtained distributions. The match in peak pitch angles at 1.4 and 1.8. $\mathrm{MA}$ is especially good, indicating the existence of a localized source of escaping CFPs, i.e. the existence of a diffusion threshold. We will get back to that point. At $0.6 \mathrm{MA}$, the match is not as good, especially at low pitch angles. However at lower pitch angles the vignetting effect would reduce the recordable light by $\sim 50 \%$ (see Sect. 3.2.2 and Fig. 3.23) and would consequently improv the agreement significantly. Some of the difference could be due also to the current profile which we know less about at 0.6 MA (no SNAP calculations available and no sawteeth present). Other effects, especially from the RF limiters will be explored later in this section.

One of the striking features observed during this current scan is the progressive appearance of a second peak. The peak in the pitch angle distribution for the firstorbit losses moves down (in angle) with plasma current. We know that the maximum in first-orbit losses occurs at the orbit which comes the closest to the plasma center which corresponds to the fattest banana orbit. As illustrated in Fig. 4.12 the fattest banana orbit (or more visually, the orbit closest to the plasma center) changes dramatically with current. At 0.6 MA the peak in first-orbit losses, calculated by ORBi $\mathrm{T}$, occurs at $\chi \approx 57^{\circ}$ and moves down to $\chi \approx 47^{\circ}$ at $1.8 \mathrm{MA}$; variation observed in the experimental results shown in Fig. 4.11. Note however, that when the optical blurring is taken in account the peak shifts upwards by approximately $3^{\circ}$. In the case of TF ripple losses, the maximum in flux corresponds to the spatial origin of the diffusing particles, not to a specific orbit. We saw previously that ripple losses should predominantly come from a region just under the magnetic axis $(\theta \approx-\pi / 2)$. That region of the plasma is connected to the outer wall (near the detector) through an 

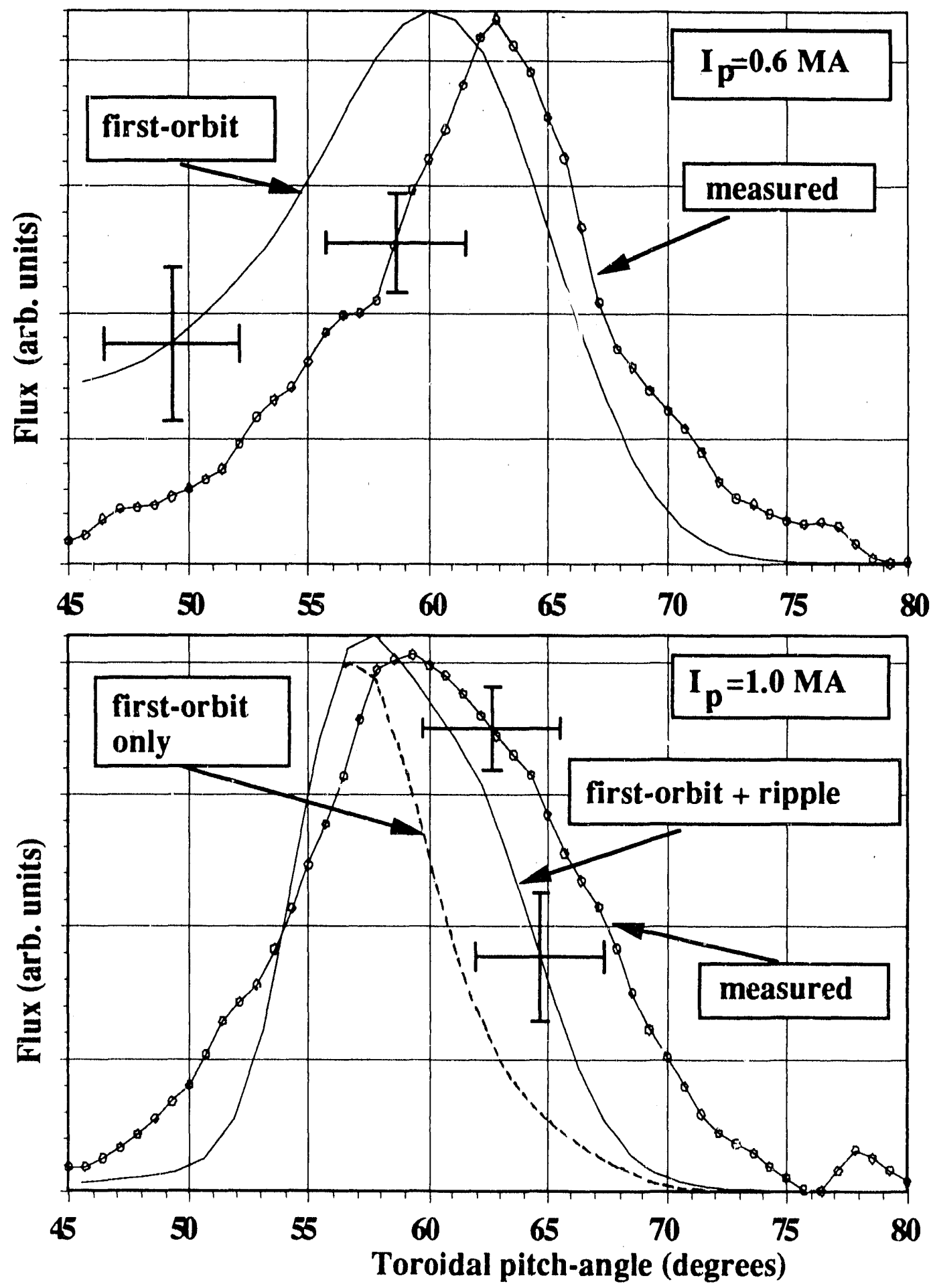

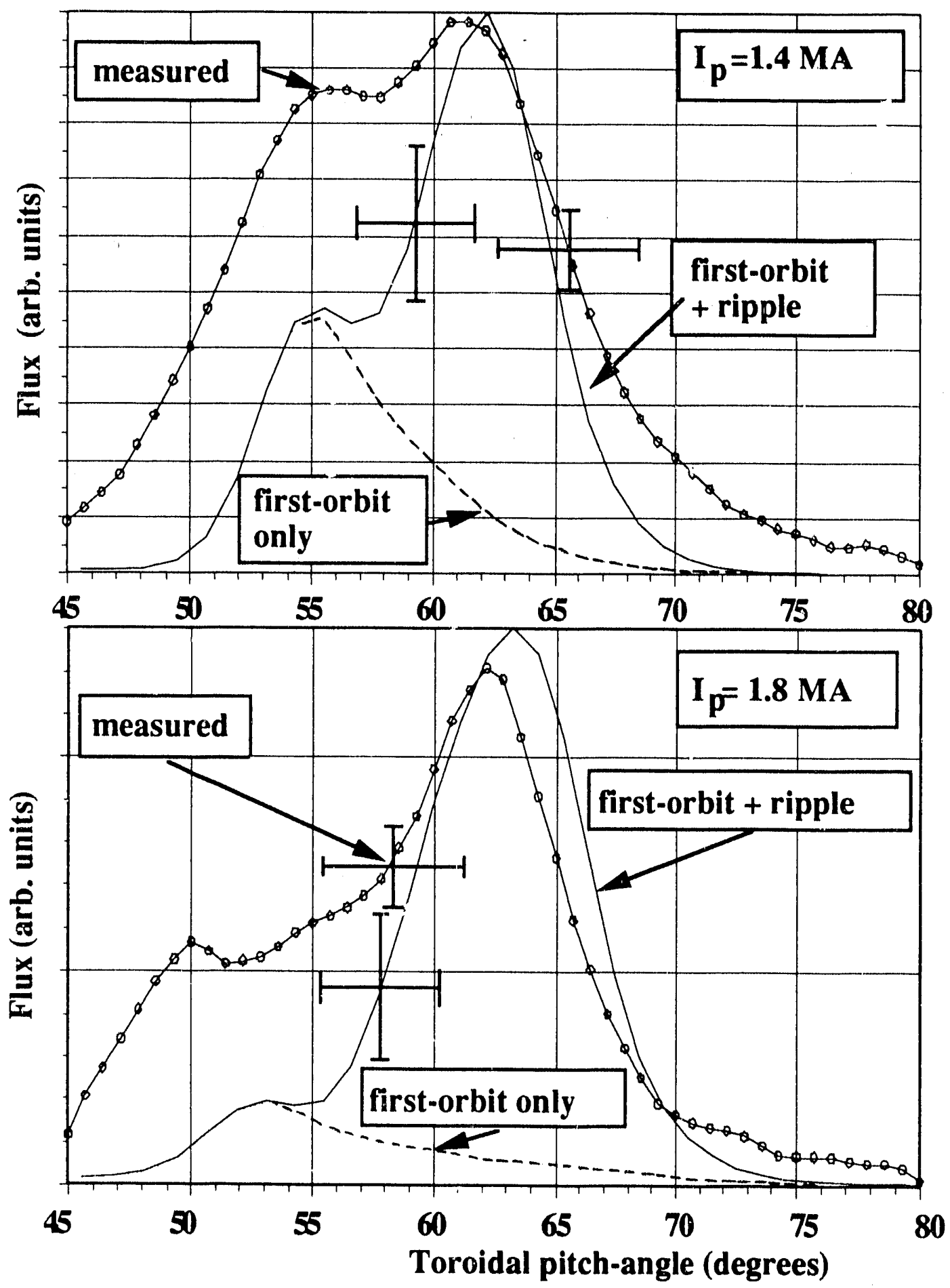

Figure 4.11: Measured pitch angle distributions for the current scan at large major radius are compared to MAPLOS calculations. 

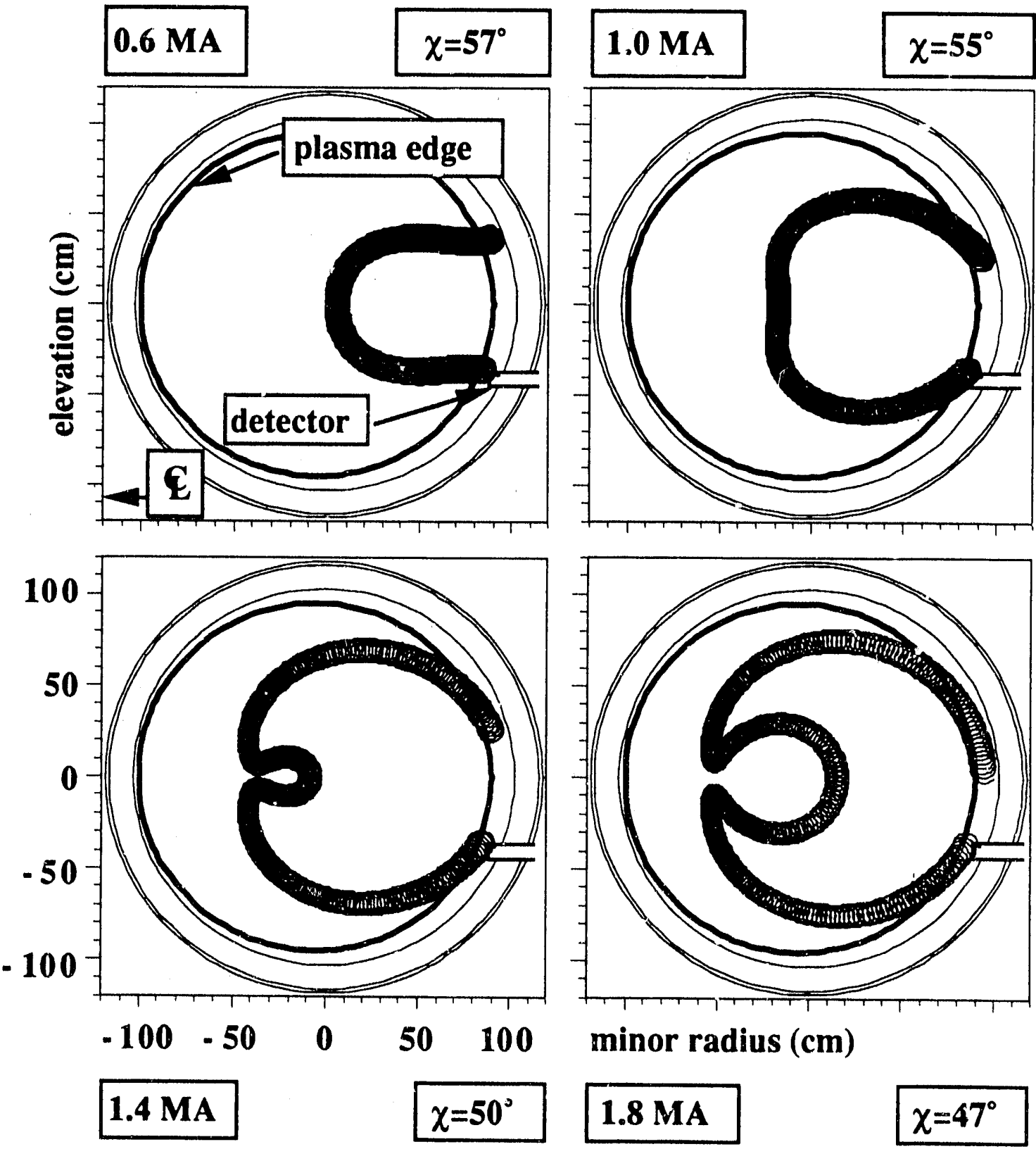

minor radius $(\mathrm{cm})$

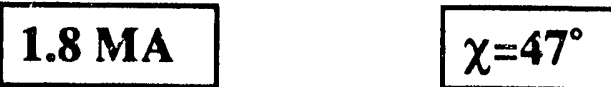

Figure 4.12: Variations of the fattest banana orbit with plasma current (with $R_{o}=2.60 \mathrm{~m}$ and $B_{T}=4 \mathrm{~T}$ ). The maximum in first-orbit losses would come from these orbits because of their closest approach to the plasma center.

orbit with a pitch angie near $60^{\circ}$ at the wall.

One more piece of evidence for the presence of TF ripple losses comes from the 
pitch angle distribution found with different plasma conditions. In Fig. 4.13 we show cases taken with a "standard" plasma, $R_{o}=2.45 \mathrm{~m}, B_{T}=5 \mathrm{~T}$ at different curreits. Again, with increasing plasma current, we expect the peak to shift down in pitch angle. In the cases shown, we see that the distributions become rapidly dominated by ripple losses above 1.2 MA. The effects of the RF limiters are also important here and will be explored after the discussion on the threshold.

One of the principal characteristics of TF ripple losses is its stochastic threshold. In the tokamak geometry, that threshold corresponds, in spatial coordinates, to a region that we called the stochastic domain (see Fig. 4.2). If that region was covering the whole plasma the majority of ripple diffusing particles would originate from the vertical chord with the maximum in density of bounce points (see Fig. 4.3). In the case shown in that figure, the maximum would correspond to the vertical chord at $r \approx 35 \mathrm{~cm}$ on the inner side of the magnetic axis. On the other hand, if the threshold was really high, the stochastic domain would be limited to a small region in the outer portion of the plasma only, i.e. at large major radius. In the latter case ripple diffusing particles would be detected at an higher pitch angle only and naturally, in less number.

We tested numerically the location of the loss domain by artificially varying the coefficient of the stochasticity threshold (Eq. 2.8). We took a coefficient of 1.0 for the threshold found by Goldston, White and Boozer. On their part, Grua and Roubin ${ }^{21}$ found a relative coefficient of 1.83 for the stochastic threshold with the same parametric dependence as Goldston, White and Boozer. Consequently, we varied the threshold coefficient and applied the calculations to the $I_{p}=1.4 \mathrm{MA}, R_{o}=2.60 \mathrm{~m}$ and $B_{T}=4 \mathrm{~T}$ case discussed above. Shown in Fig. 4.14, are the results of the simulated pitch angle distributions compared with the one obtained experimentally (bottom). All parameters of the calculations were kept the same except for the threshold coefficient which was varied from 0.3 to 5 . The changes in the distribution are twofold. The first change, with increasing coefficient, is the progressive appearance of the first-orbit peak (left) which will eventually dominate. The second is the pitch angle at which ripple losses peak. In the first graph they peak at $59^{\circ}$, whereas at high threshold coefficient they peak at $65^{\circ}$, a trend that we described above using arguments based on the domain location. Compared to the experimental results, we conclude that within the uncertainty of this measurement and calculations, the threshold coefficient must be between 1.0 and 3.0 consistent with the theoretical expectations. It is also inter- 


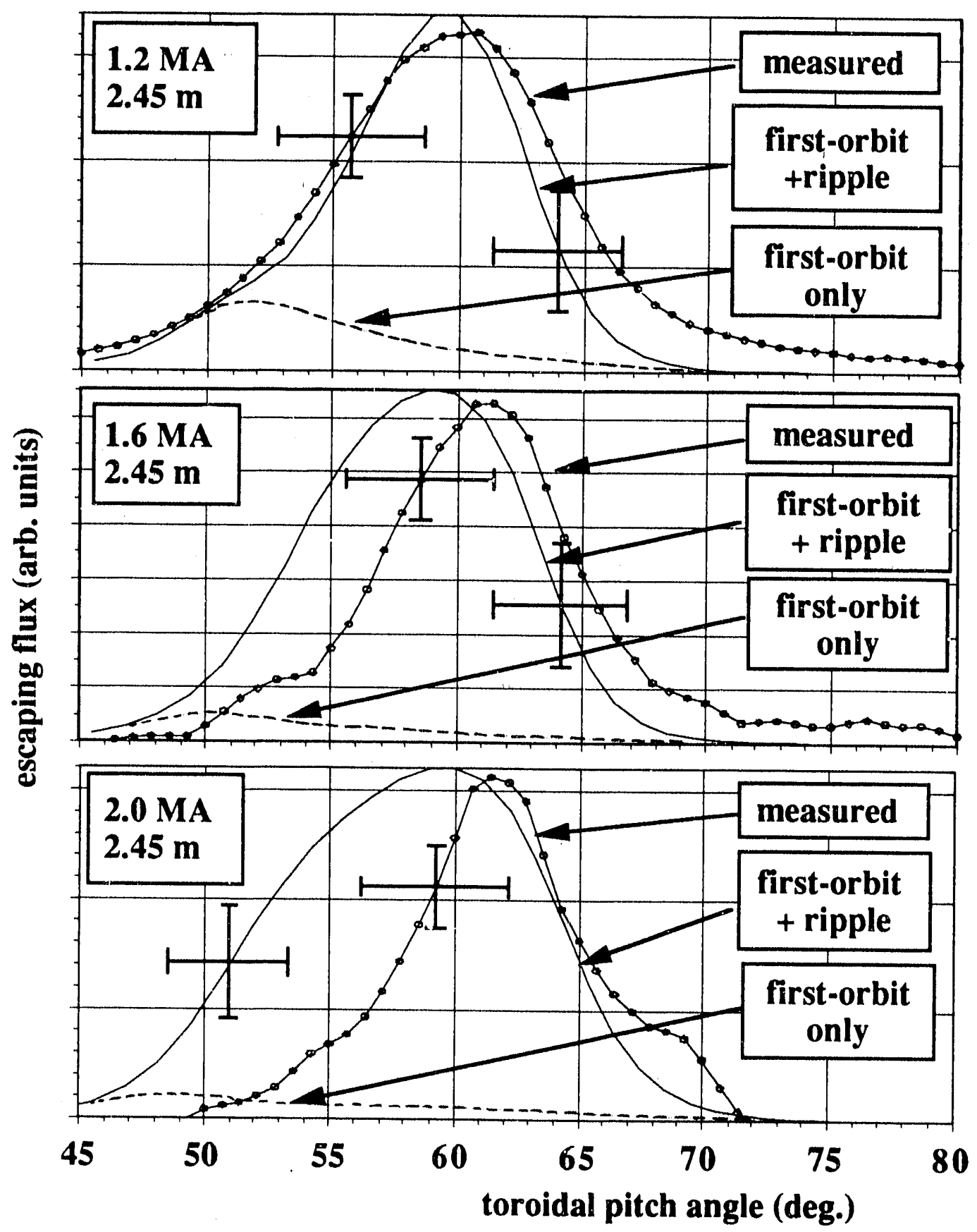

Figure 4.13: Comparisons of measured pitch angle distributions with numerical calculations for various plasma currents in a "standard" plasma $\left(B_{T}=5 T, R_{\mathrm{o}}=2.45 \mathrm{~m}\right)$. 
esting to notice that the confinement of trapped particles (excluding the first-orbit lost (FPs) changes from $56 \%$ losses (at a coefficient of 0.3 ) to $1 \%$ only (at 5.0 ).

The amplitude of the different components is also subject to numerous uncertainties. They are essentially the same as the ones discussed in the previous section, especially when looking at the effects of different source and current profiles. The relative ratio of the ripple to the first-orbit losses is particularly interesting. We observed that the relative amplitude of the peaks was changing slightly versus time, during a discharge. That change could be the effect of changing profiles with or without the contribution of sawteeth. Unfortunately the time resolution during these shots was not sufficiently good to allow a possible correlation of the relative amplitude of the two peaks with sawteeth activity.

However, as we mentioned in the preceding sections the predominan uncertainty in the numerical simulations lie in the description of the first wall geometry, especially with the RF limiters. We added the limiters to MAPLOS and positioned the first wall at the location of the detector's isperture. Statistics for the scintillator were however reduced since the geometry was no longer axisymmetric. We show in Fig. 4.15 some of the cases presented above with now the RF limiters added to the numerical simulations. Low current cases, which were dominated by first-orbit losses were naturally not affected by the change and are not shown. The interesting change comes in the $R_{o}=2.60 \mathrm{~m}$ case, at $I_{p}=1.4 \mathrm{MA}$ and $1.8 \mathrm{MA}$ where the ripple peak is reduced (partly blocked by the limiters), improving significantly the agreement between the reasured and calculated distributions. Similar improvements in the agreement can also be found in the $R_{o}=2.45 \mathrm{~m}$ case as shown in Fig. 4.15 .

We investigated experimentally the effects of the RF limiters by changing slightly the detector position behind the RF limiters, in conditions very similar to the $\boldsymbol{R}_{\mathbf{o}}=$ $2.60 \mathrm{~m}, I_{p}=1.4 \mathrm{MA}$ case. In fact only the toroidal field was modified (from $4 \mathrm{~T}$ to $3.5 \mathrm{~T}$ ) which does not change the confinement significantly. We show in Fig. 4.16 the variation of the pitch angle distribution as the aperture was moved gradually away from the wall towards the RF limiters (over $3.5 \mathrm{~cm}$ ). Also shown are the numerical calculations (including the presence of the RF limiters) made for each case. As pitch angle distributions are calculated at the wall only (no detector), we simply located the first wall at the radial location of the aperture. All the measured distributions have the same normalization (neutron normalized and same camera gain). We see that the first-orbit peak increases very little whereas the ripple peak at least doubles 


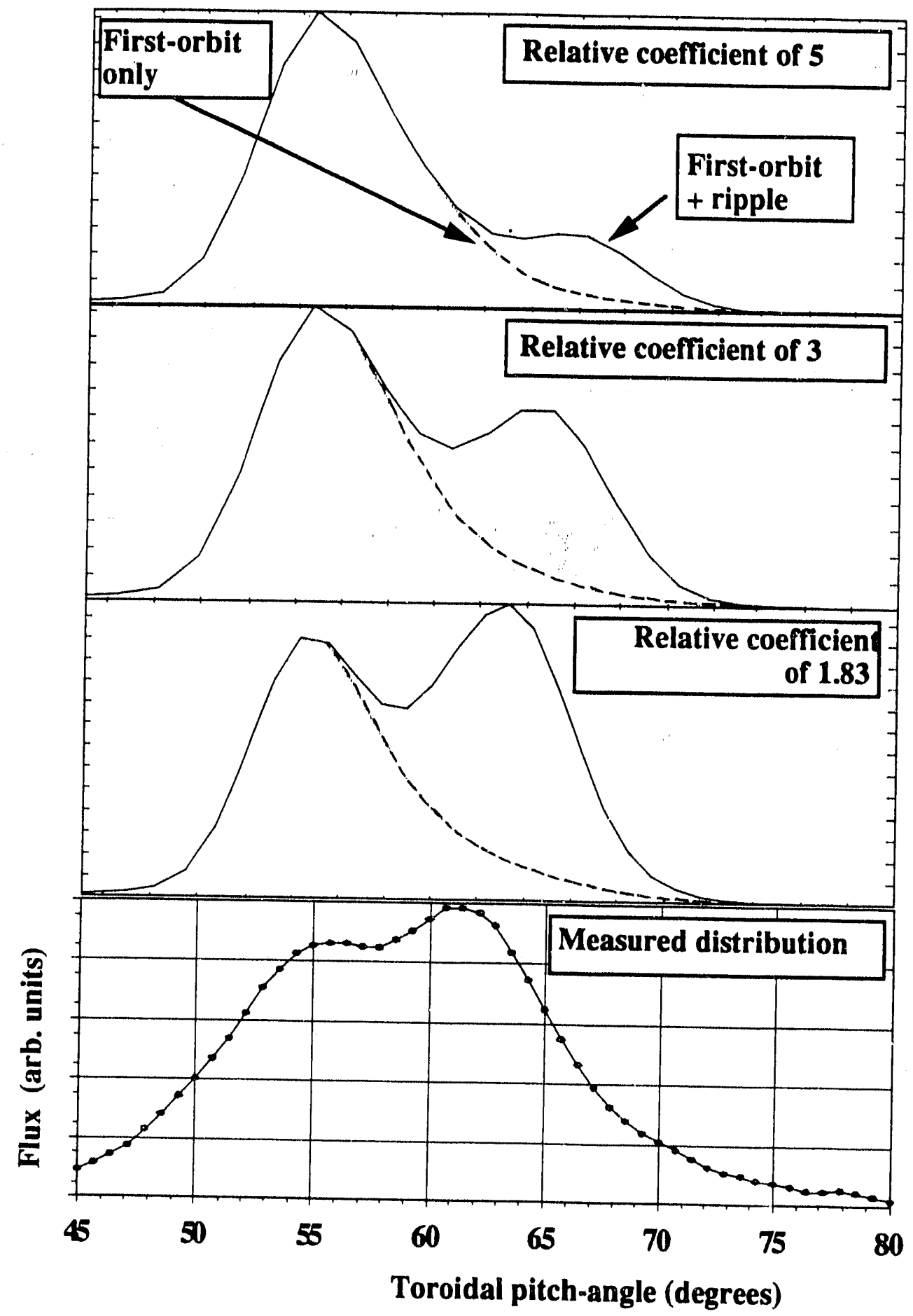




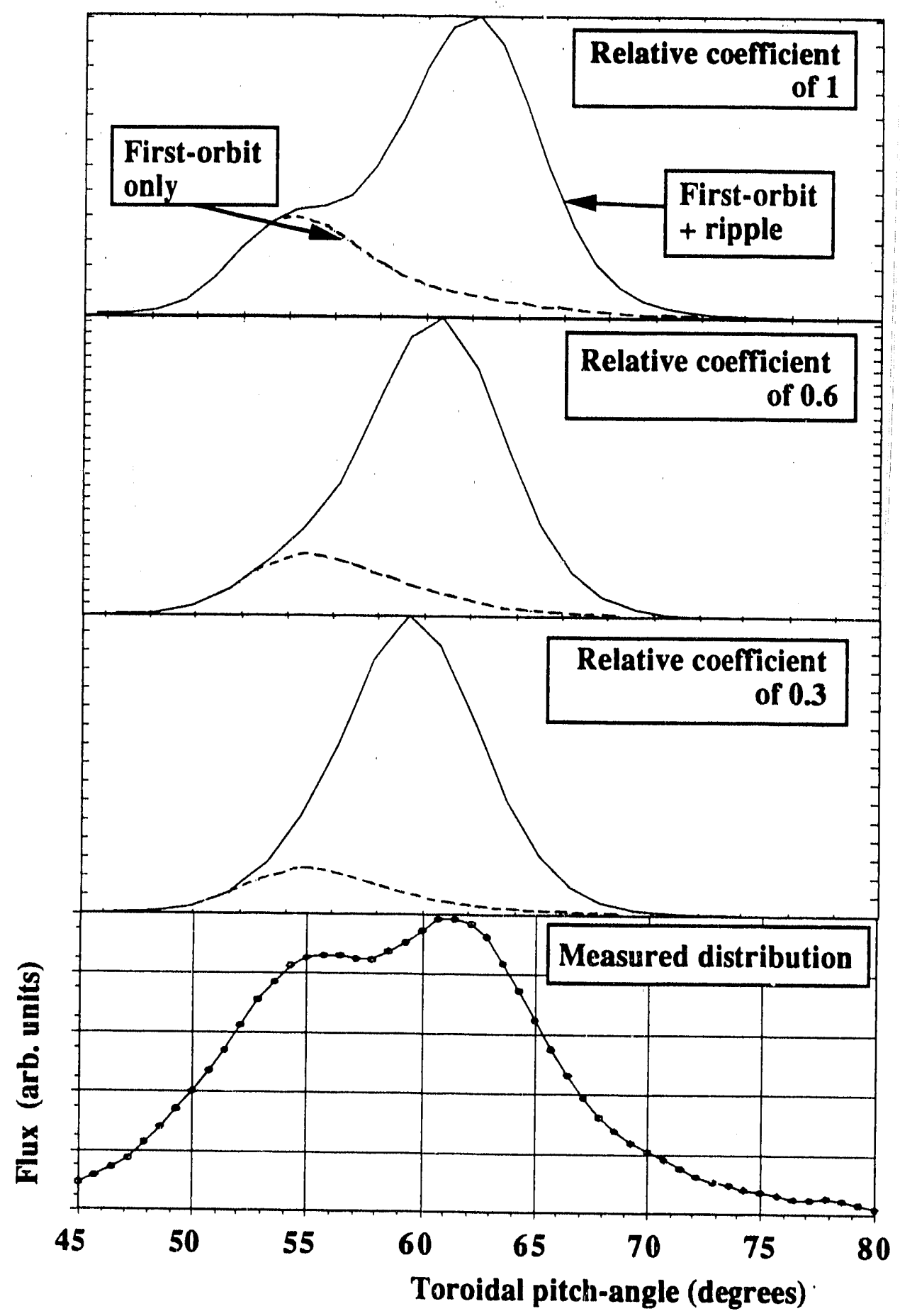

Figure 4.14: Effects of the variation of the stochastic threshold coefficient on the pitch angle distribution. The Goldston, White and Boozer threshold has a relative coefficient of 1.0 . The coefficient was varied from 0.3 to 5 , all other parameters were kept constant. 


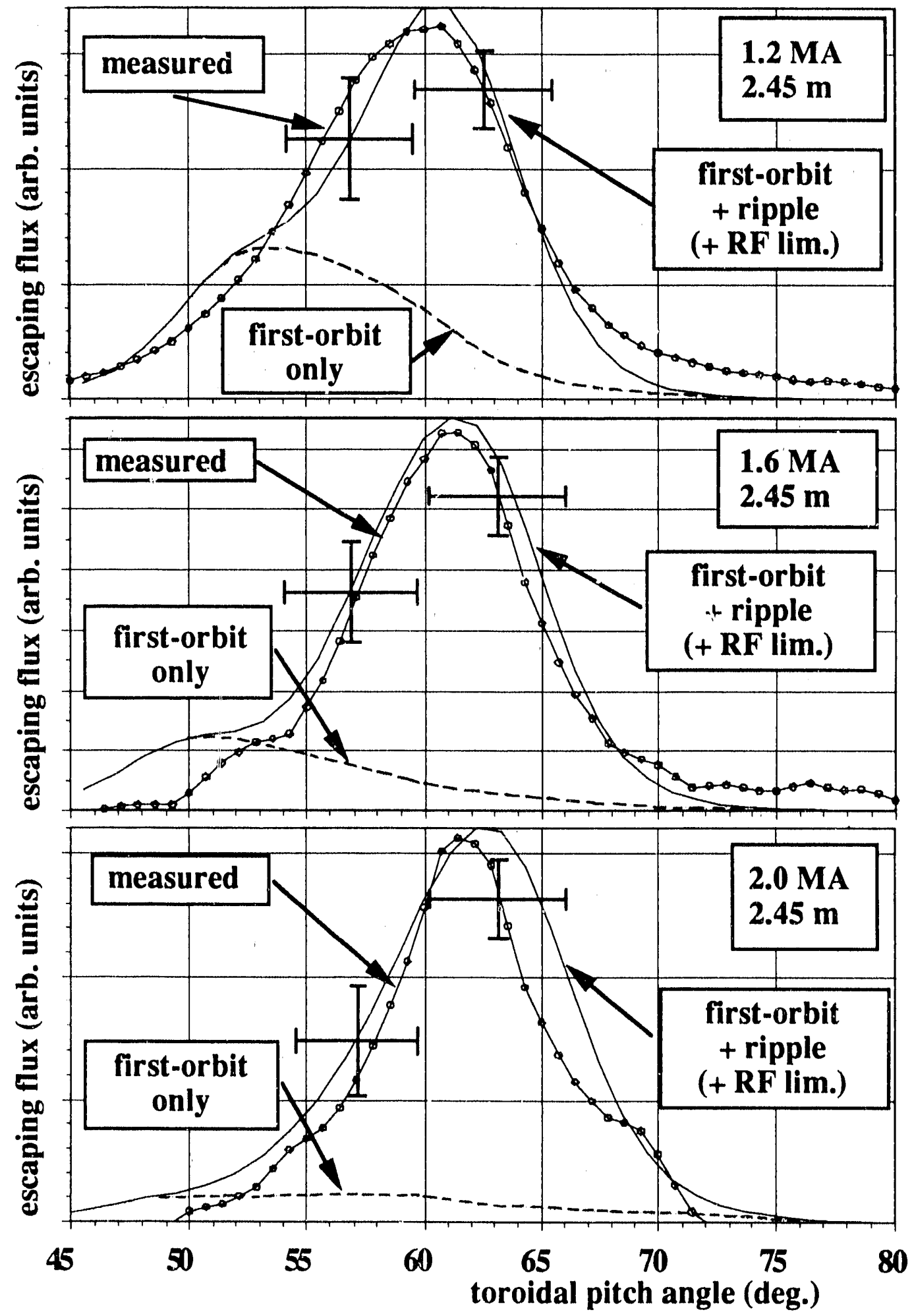



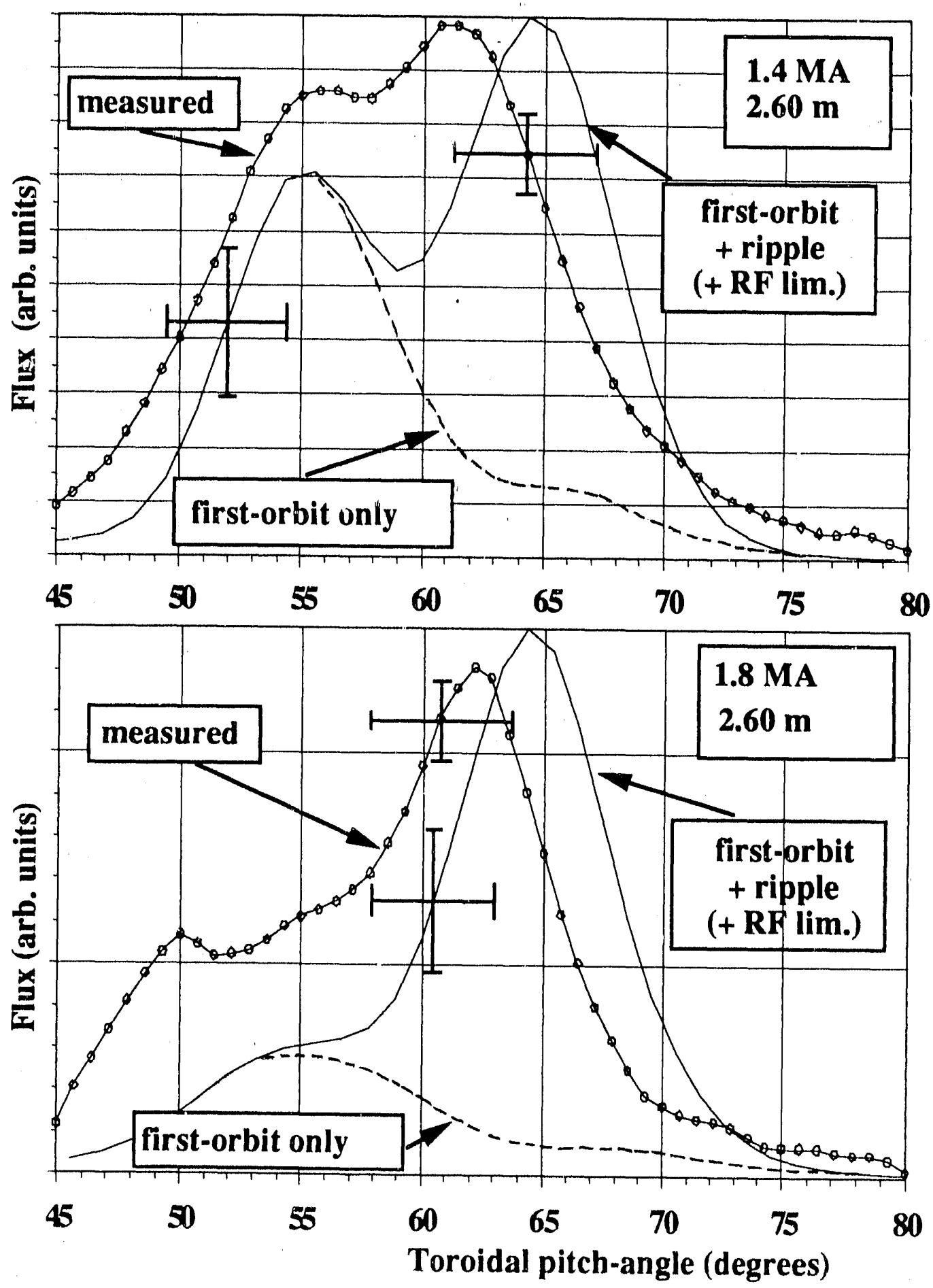

Figure 4.15: Comparisons of the measured pitch angle distributions with numerical calculations incluaing the RF limiters. The first wall is located at the detector's aperture position. 
in amplitude as we bring the aperture further in the vacuum vessel (or equivalently closer to the radius of the RF limiters). This difference in behavior is characteristic of a first-orbit and diffusing mechanisms. Ripple diffusing particles have a larger probability of being scraped off by the limiters because of their small step size $(\sim 1$ $\mathrm{cm}$ ) whereas first-orbit lost particles are are directly lost to the wall and are much less affected by the limiters (with the aperture located behind the RF limiters). These effects will be discussed in more details in Ch. 5 and are illustorated in Fig. 5.8 .

\subsubsection{Poloidal distribution}

One of the main motivations for the study of TF ripple losses was to look for the possibility of highly localized losses just below the outer midplane of TFTR. The main difficulty in that task lies in the limited number of detectors that one can put on the first wall, which in our case, was a total of four detectors. However by looking at Fig. 4.6 we notice that by the presence of the midplane detector we are in a very good position to look for the presence or not of ripple losses. In the case shown in Fig. 4.6 we see that ripple losses account for $\sim 80 \%$ of the losses at $20^{\circ}$ below the midplane. Such a big difference would be distinguishable when compared with the bottom detectors. However, the comparison of the experimental results with the numerical simulations is complicated by certain considerations, mainly geometrical.

The first consideration resides in the small differences between the detectors. As discussed in Sect. 3.2.2 the four detectors have different optical efficiencies that we measured by cross-calibrating them with a light source. The results of crosscalibration were reported in Table 3.1. To that optical cross-calibration we have to add the effects of differences in the apertures of the detectors. The three bottom detectors have identical pin-hole and slit dirnensions (within $15 \%$ of uncertainty) but the midplane detector has smaller pinhole and slit and so the measured flux must be corrected upwards by $40 \%$, which is the difference in areas from the two system of apertures. One of the major uncertainty in these calculations comes from the calculations of the loss distribution; MAPLOS maps the losses on the first wall, not at the detectors themselves. In reality the detectors are somewhat sticking out from the first wall (especially for the midplane detector where the RF limiters add to the uncertainty). At the very bottom of the machine the difference would be quite small since the orbits come nearly perpendicular to the wall, whereas at the midplane they 


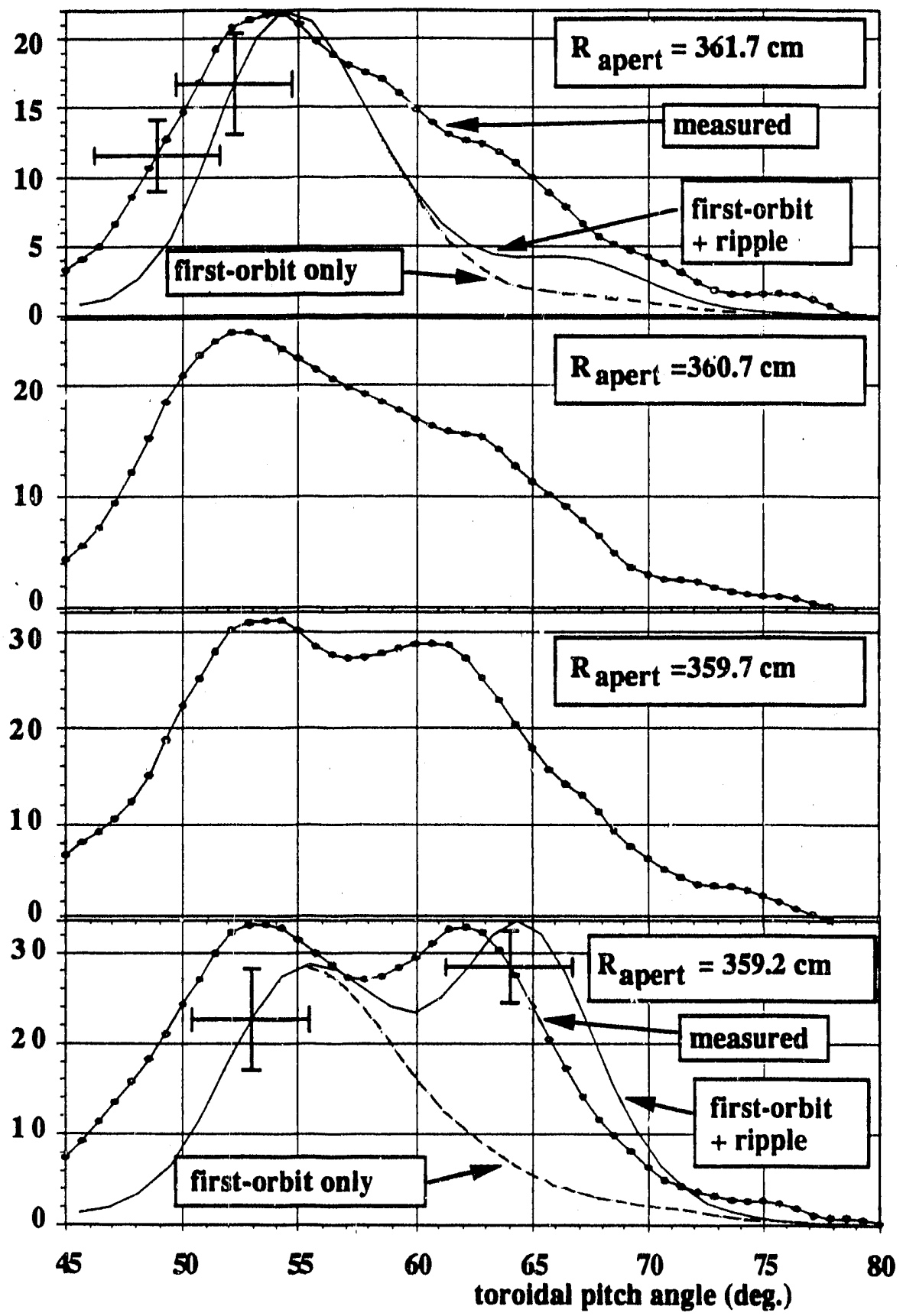

Figure 4.16: Pitch angle distributions obtained with decreasing radial positions of the aprerture behind the RF limiters. The experimental results have been normalized by the neutron produciion and the camera gain remained the same. The ripple peak (right) doubles in amplitude whereas the first-orbit peak increases only slightly. 
impact more tangentially. In the latter case one must consider the orbital effects on the calculated flux.

In Fig. 4.17 are shown cases taken at different plasma currents. At low current $(0.6 \mathrm{MA})$ the calculated distribution is dominated by first-orbit losses widely spread at the bottom of the vacuum vessel, whereas at high current (1.8 MA) the distribution is very largely dominated by ripple losses localized below the midplane. To the numerical curves we added the points corre ${ }_{s}$ onding to the different detectors. The experimental points were normalized to the model curve at detector \#6, located at $\theta=90^{\circ}$. Also indicated are the uncertainties in the numerical calculations which were obtained by varying the source and current profiles over a range consistent with the SNAP ralculations and source profile measurements.

We see that for the current scan shown $\left(R_{o}=2.60 \mathrm{~m}\right)$ we have a semi-quantitative agreement, especially if we consider the extremes (detectors at $-20^{\circ}$ and $-90^{\circ}$ ). Unfortunately for the other two detectors (and especially the one at $-45^{\circ}$ ), the scintillator impact location of the CFPs was too ciose to the detector inner walls (this effect is mainly due to the lower magnetic field used, which translated into a larger gyroradius). In fact a significant fraction was probably out of the scintillator range gyroradius range. This is more so at higher current when the peak in first orbit losses move to lower pitch angles, closer to the stainless steel casing.

\subsubsection{Absolute flux of CFPs}

So far, we compared the experimental results with the numerical calculations by normalizing the different ciistributions at relatively arbitrary points. However, in spite of the uncertain absolute calibration that we described in Sect. 3.2.2, we can still look at the presence of anomalous losses above the first-orbit level. Contrary to MAPLOS, which maps losses to the wall, ORBIT calculates the losses to the detector taking into account its exact dimensions (i.e. the aperture and slit). Naturally ORBIT is not equipped to look at ripple losses and a correspondence must be established between the two codes to permit a complete calculation.

The efficiency of the detector (the detected fraction of the total production of CFPs) is a function of both the detected particle orbits and the solid angle viewed by the slit and aperture. ${ }^{45,47,52}$ Some details of those calculations are reviewed in App. A.3. In Table 4.1 we listed the results of a comparison between measured and 


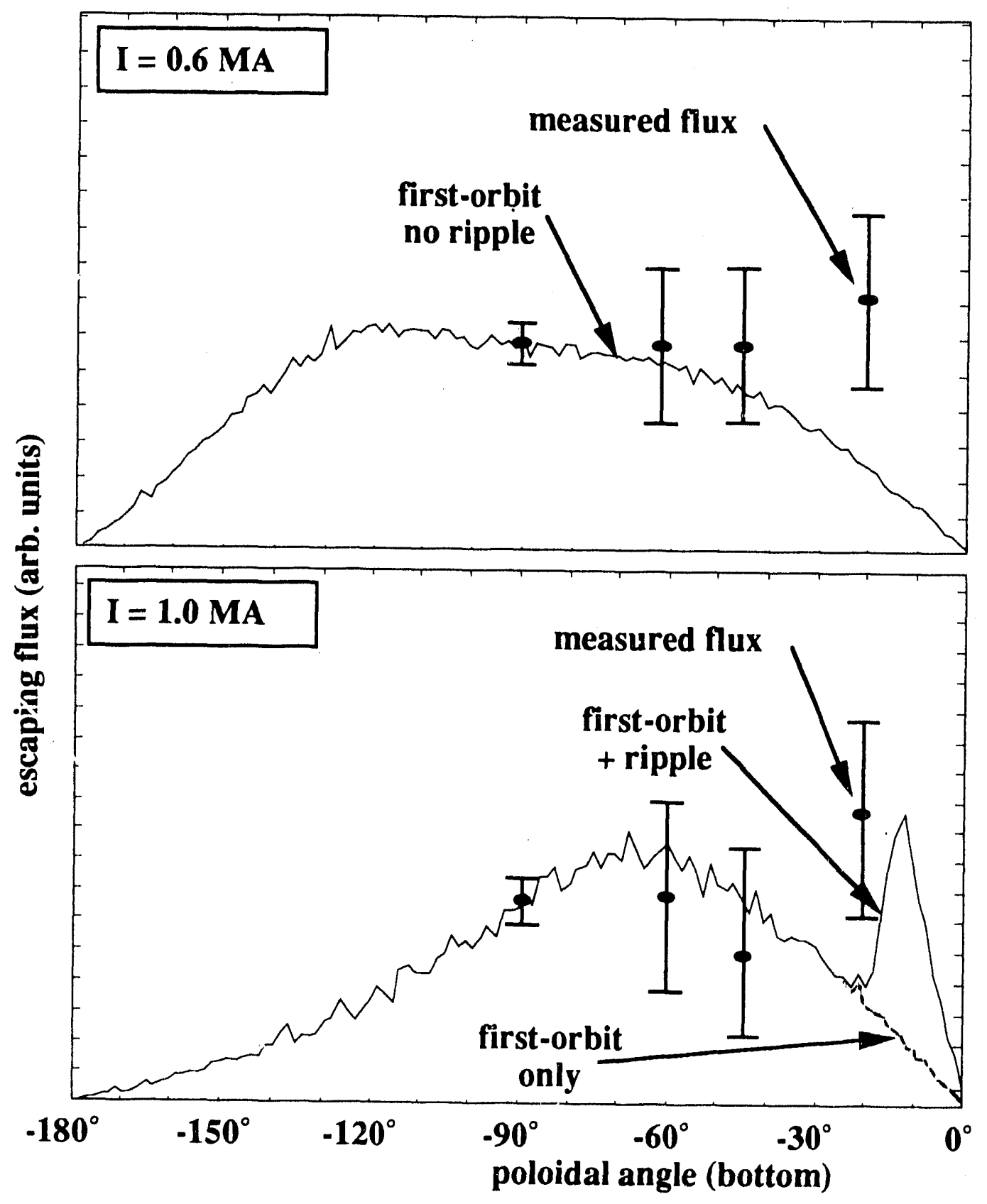




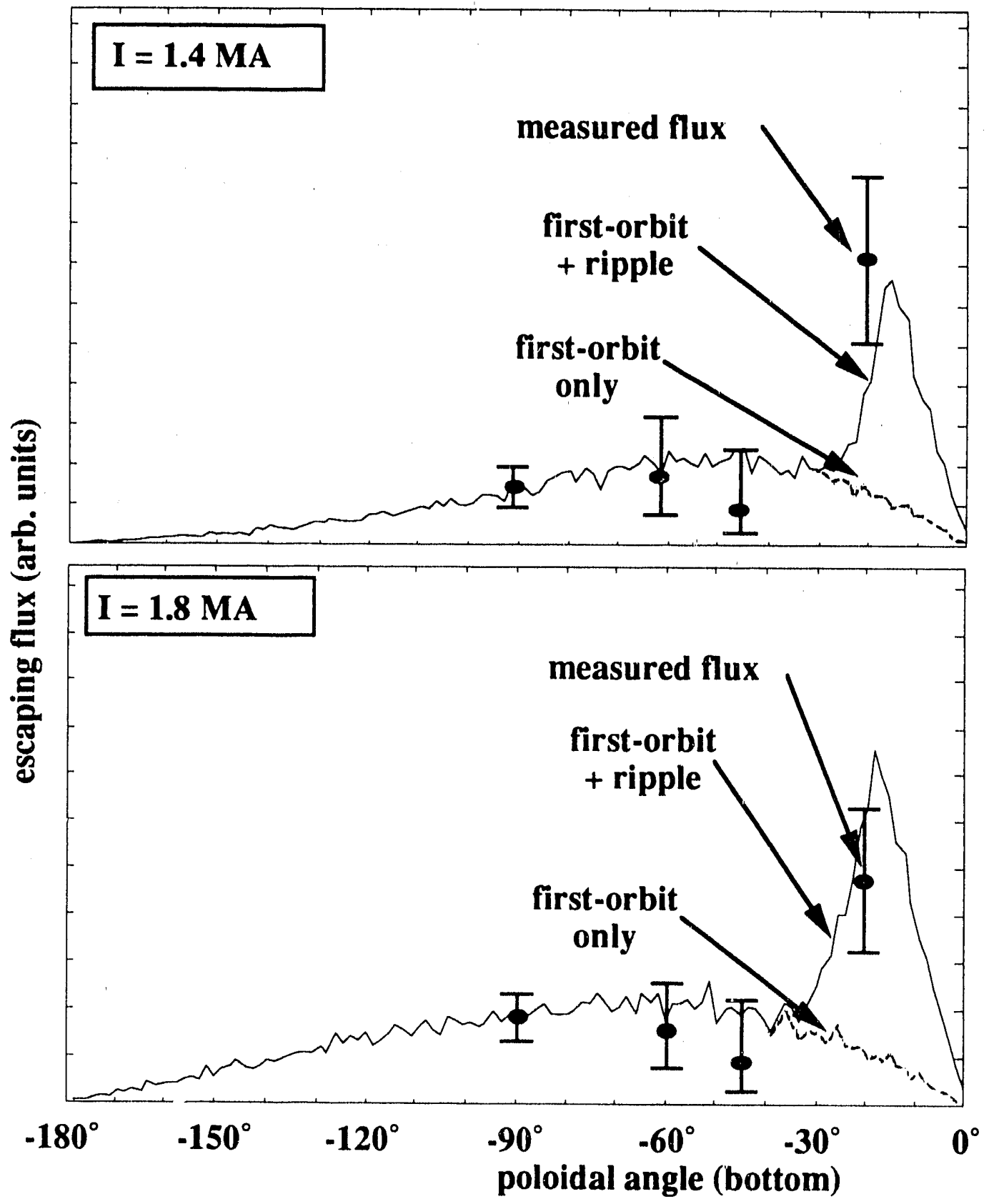

Figure 4.17: Poloidal distribution of CFPs at the bottom of TFTR for different plasma currents $\left(B_{T}=4 T, R_{o}=2.60 \mathrm{~m}\right)$. To the numerical calculated distribution (continuous line) we added the experimental points obtained with the four detectors. The experimental points are normalized at $\theta=-90^{\circ}$. 


\begin{tabular}{||c|c|c|c|c||}
\hline$I_{p}(M A)$ & $R_{o}(m)$ & $B_{T}(\mathrm{~T})$ & Calc. efficiency $( \pm 50 \%)$ & Meas. efficiency $( \pm 80 \%)$ \\
\hline 0.6 & 2.60 & 4 & $\frac{6.0 \times 10^{-8}}{3.6 \times 10^{-8}}$ & $1.9 \times 10^{-8}$ \\
\hline 1.0 & 2.60 & 4 & $1.6 \times 10^{-8}$ & $1.6 \times 10^{-8}$ \\
\hline 1.4 & 2.60 & 4 & $7.2 \times 10^{-9}$ & $3.1 \times 10^{-8}$ \\
\hline 1.8 & 2.60 & 4 & $6.4 \times 10^{-8}$ & $1.1 \times 10^{-8}$ \\
\hline 0.6 & 2.45 & 5 & $5.2 \times 10^{-8}$ & $1.4 \times 10^{-8}$ \\
\hline 0.8 & 2.45 & 5 & $3.8 \times 10^{-8}$ & $10.5 \times 10^{-8}$ \\
\hline 1.0 & 2.45 & 5 & $2.2 \times 10^{-8}$ & $5.7 \times 10^{-8}$ \\
\hline 1.2 & 2.45 & 5 & $1.4 \times 10^{-8}$ & $4.6 \times 10^{-8}$ \\
\hline 1.4 & 2.45 & 5 & $8.5 \times 10^{-9}$ & $2.0 \times 10^{-8}$ \\
\hline 1.0 & 2.45 & 5 & $1.0 \times 10^{-8}$ & $6.4 \times 10^{-9}$ \\
\hline 1.8 & 2.45 & 5 & $8.0 \times 10^{-9}$ & $2.3 \times 10^{-9}$ \\
\hline 2.0 & 2.45 & 5 & & $1.3 \times 10^{-9}$ \\
\hline
\end{tabular}

Table 4.1: Comparison of the calculated (first-orbit only) and measured absolute efficiencies (三 flux/neutron) to the detector for various plasma conditions. Source profiles width were taken from the neutron multi-channel collimator.

calculated flux at the midplane detector. The flux measured at the detector has been obtained by taking the average flux over the CFP impact surface of the scintillator. We see here that the measured fluxes are in the same range than the expected ones. Unfortunately, as we mentioned before, the obtained precision (both in numerical and measured flux) is not sufficiently good to permit to distinguish between loss mechanisms using this measurement alone.

\subsubsection{Energy distribution}

As described with more detail in Sect. 3.1.1 the detector can, with its pinhole and slit, discriminate escaping particles by their gyroradii $(\rho)$. Knowing $\rho$, the energy of the particles can be recovered (neglecting the poloidal field in the expression for $|B|$ ) by using:

$$
\rho=\frac{v_{\perp}}{\Omega}
$$


where

$$
v_{\perp}=\sqrt{\frac{2 E}{m}} \sin \chi
$$

and

$$
\Omega=\frac{q B}{m} .
$$

Here $m$ and $q$ are the mass and the charge of the CFP and $\chi$ is the toroidal pitch angle (see Sect 2.1). The study of the energy is complicated by many factors, some regarding the detector and others, regarding the physical interpretation. As shown in Fig. 3.10 the energy resolution is poor and the energy dependence of the scintillator efficiency must be taken in account (see Fig. 3.19). The gyroradii of the $1 \mathrm{MeV}$ triton and the $3 \mathrm{MeV}$ proton are also slightly different $(\sim 10 \%)$ after the internal $3 \mu \mathrm{m}$ aluminum foil. However, we can compare with fair accuracy, distributions from different plasma conditions, without knowing with precision the energy correspondence. The time dependence of the energy distribution can also be followed in order to study the possible presence of a time delayed loss mechanism.

All these measurements are complicated by the fusion mechanism itself. In TFTR as in other machines of the same type most of the fusion reactions are produced by the injection of energetic neutrals (NBI). In most conditions the CFPs are predominantly produced by beam-beam and beam-target reactions ( $Z 80 \%$ ). The relatively large energy of the beam ions $(\sim 100 \mathrm{keV})$ and also the difference in energy between them and the thermal ions $(\sim 10 \mathrm{keV})$, would both contribute to a substantial Doppler shift in the energy of the CFPs; more so for the $1 \mathrm{MeV}$ triton (between $0.7 \mathrm{MeV}$ and $1.5 \mathrm{MeV}^{109}$ for $100 \mathrm{keV}$ beam ions, with a FWHM in the shift of approximately 400 $\mathrm{keV}$ ) than for the $3 \mathrm{MeV}$ proton $(2.7 \mathrm{MeV}-3.4 \mathrm{MeV})$. The relatively large difference (compared to the $100 \mathrm{keV}$ of the beam ions) results from the fact that we add (or subtract) the velocities not the energies. The birth cnergy distribution is even more complicated by the fact that the neutrals are not injected at the same energy $(\sim$ $20 \%$ spread for the full energy component for TFTR ) and by the presence of half and third energy components from injected deuterium molecules $\left(\mathrm{D}_{2}^{+}\right.$and $\left.\mathrm{D}_{3}^{+}\right)$. The use of a neutron spectrometer could help substantially in measuring the birth energy distribution but was not available for our experiments. The Doppler shift has also a modest effect on the isotropy of the fusion products distribution. All those effects must be included before demonstrating the possible presence of escaping slowed-down particles. One must rely more on the other capabilities of the detector to do so. 
With those caveats in mind, one can still obtain some information from the gyroradius distribution, at least the location of the peak in the energy distribution of the escaping particles. In Fig. 4.18 is shown the measured distribution for a $I_{p}=$ 0.8 and $1.6 \mathrm{MA}, B_{T}=5 \mathrm{~T}$ and $R_{o}=2.45 \mathrm{~m}$ discharge. The measured escaping flux distribution peaks at $\rho=7.3 \mathrm{~cm}$ corresponding to an energy of $1 \mathrm{MeV}$ for the triton or $3 \mathrm{MeV}$ for the proton. Also shown is a numerically calculated distribution (using the orbit code mentioned in Sect. 3.1.1) for tritons and protons escaping with their birth energy (monoenergetic flux) and the same pitch angle. The calculated distribution also includes the effects of optical broadening as discussed in Sect. 3.2 .2 but not the effects due to vignetting. The peaks in the distributions agree fairly well, which is consistent with theoretical expectations. As for the width of the distribution, we observed a broader distribution (with an additional flux at lower gyroradius) than the numerical calculations. The difference is certainly due, at least in part, from the birth energy distribution which is not monoenergetic mainly because of the Doppler shift induced by the beam ions. In this case the vignettting effects must be included in order to study further the energy distribution (see Fig. 3.23).

\subsection{Other experiments}

As discussed briefly in Sect. 4.2, there are many possible ways of looking at TF ripple losses, in the previous chapter we only used the current scan. Naturally the other possibilities are not necessarily easier. Most of the results presented in this section were obtained in piggy-back mode, the experiment being planned for another purpose or study. In most cases the results are fragmentary, at least for our purpose, but nevertheless present very interesting features worth presenting.

The first experiment is related with the previous section in that the plasma current is varied, but here not from a shot to shot basis but dynamically during a shot, namely by ramping the current (in our case ramping down). The second experiment involves plasma compression, with the possibility of varying dynamically the ripple losses by bringing the plasma to a lower ripple amplitude. Finally, we will explore the effects of neutral beam power on the confinement of CFPs, both for the amplitude of losses near the midplane and for its time dependence.

The results of these experiments are presented without a full analysis, in some cases because of the lack of a complete experimental characterization, and in all of 

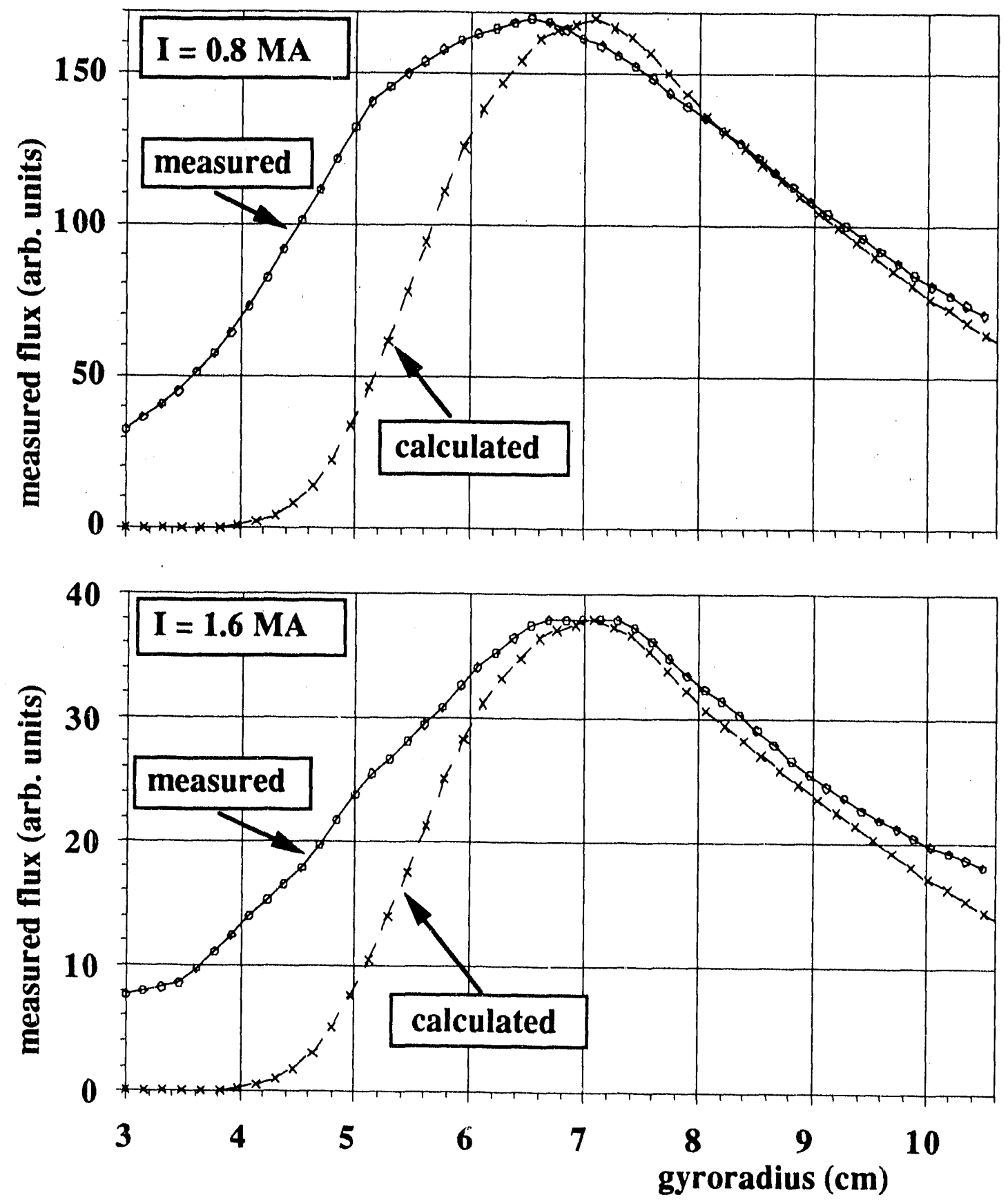

Figure 4.18: Comparisons of gyroradius distributions for two different plasma currents (0.8 and 1.6 MA with $R_{\mathrm{o}}=2.45 \mathrm{~m}$ and $\left.B_{T}=5 \mathrm{~T}\right)$. Also shown are the numerical distributions for a monoenergetic escaping flux (at $7.3 \mathrm{~cm}$ in gyroradius), which include the effects due to optical broadening. 
them because some of the theoretical tools are missing or presently inadequate. The common trait in those results reside in their time dependency, complicating extensively the numerical simulations. The effects on the current profile are especially difficult to obtain since we have no direct measurements. In the case of the compression the constants of motion are not necessarily conserved and a more general approach is needed, as we will discuss in the next few sections.

\subsubsection{Current rarnp experiment}

Current ramp experiments are presently used for the study of many aspects of a discharge. The fundamental one is certainly the study of the plasma current penetration. They are often used to study transport and its current scaling ${ }^{126}$ for example. In our case they affect the confinement of CFPs in a more or less predictable way, at least for the problem of first-orbit losses. In TFTR the current penetration time is of the order of 3-5 sec, long compared to the slowing down time of the CFPs. As we saw in Eq. 2.8, the threshold for TF ripple losses is a strong function of the current profile, more than for first-orbit losses, mainly because of its $q$ ' dependence. The principal difficulty comes from the total two seconds (compared to the 3-5 sec of time penetration) window for neutral beam injection. For most plasma conditions the effect of the ramp would reach the ripple threshold region (typically around half the minor radius or less) only towards the end of the beam injection.

In Fig. 4.19 we show the measured flux of CFPs for two different aperture positions. In those shots the plasma current was ramped down from 2 to $1 \mathrm{MA}$. In the bottom case the aperture is located behind the RF limiters radius where we observed a large increase in flux with decreasing current. The increase is not as dramatic in the top case where the aperture was located inside the radius of the RF limiters. Also shown is the neutron emission (dashed line) which is normalized to the measured flux just before the end of NBI $(t=4.97 \mathrm{sec})$.

\subsubsection{Plasma compression}

During plasma motion and especially during a compression it is possible to vary considerably the ripple amplitude over the plasma cross-section mainly because of its exponential radial dependence. In this experiment the plasma is compressed on the bumper limiter from a major radius of $2.45 \mathrm{~m}$ (minor radius $80 \mathrm{~cm}$ ) to $2.30 \mathrm{~m}$ 


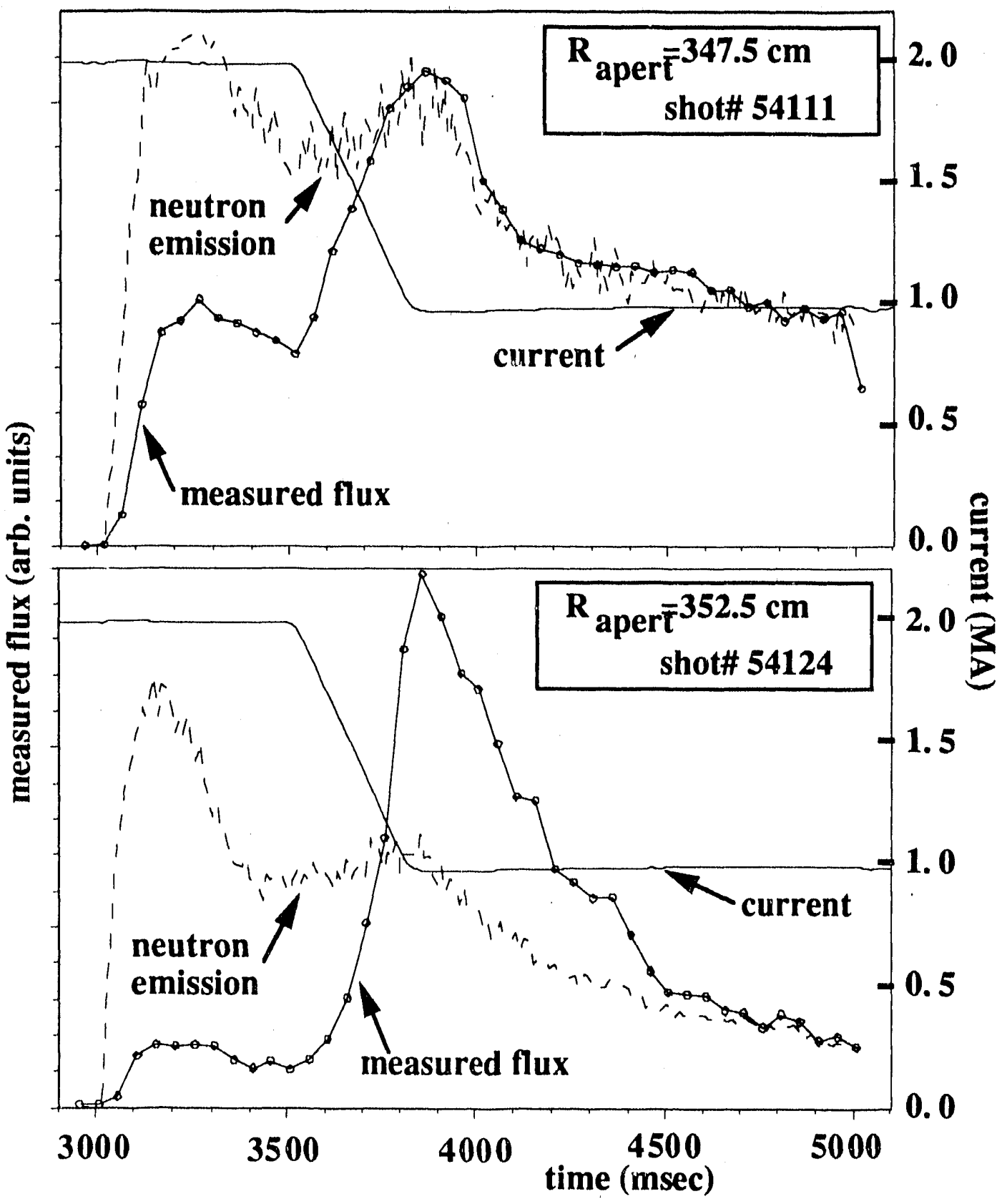

Figure 4.19: Effects of a plasma current ramp on the measured flux of CFPs for two different aperture positions. The current was ramped down from 2 to $1 \mathrm{MA}$. Also shown is the neutron emission normalized at $t=4970 \mathrm{msec}$. 
(minor radius $65 \mathrm{~cm}$ ). In general the analysis of the confinement of CFPs during the compression is arduous since the profiles are changing and the energy of the particles is not conserved. However, in our case, the compression is slow enough $(\sim 50 \mathrm{msec})$ that the motion is adiabatic, the magnetic moment $\mu$ and the parallel invariant $J_{\|}=e \psi / 2 \pi m c-v_{\|} R$ ( $\psi$ is the poloidal flux function) are conserved, not the energy. ${ }^{127}$

In Fig. 4.20 is shown the time dependence of the total escaping flux as measured by the midplane detector during the compression $\left(B_{T}=5.1 \mathrm{~T}, I_{p}=1.7 \mathrm{MA}\right.$ ). Also plotted with a dash line is the major radius position of the plasma, moving from $2.45 \mathrm{~m}$ to $2.30 \mathrm{~m}$. The CFP loss level recorded after the compression was always buried in the background level so depending on the detector position the drop after the compression varicd from a factor of 2 , when furthest out behind the RF limiters, to a factor of at least 100 or more when inside the radius of the RF limiters. Since this is a time dependent problem - the beams were shut off only 300 msec after the compression was completed -- our numerical methods are inadequate. However, if we assume a steady-state, we found that for first-orbit losses only, the flux to the detector should decrease by a factor of 5 for a range of aperture positions behind the RF limiters including the case shown in Fig. 4.20. No similar estimates could be derived easily with ripple losses since MAPLOS is not equiped for attacking this type of problems, as yet! However a large decrease is expected since the plasma is moving into a smaller ripple region.

\subsubsection{Beam power dependence}

Previous measurements of CFP losses obtained from the bottom detector (\#6) showed that the escaping flux (normalized to the total neutron production) was independent of the beam power. ${ }^{91}$ This observation was made for discharges with a major radius of $2.45 \mathrm{~m}$ or more, and is consistent with a first-orbit loss process. For the midplane detector quite the opposite was found; losses were correlated with beam power in many different conditions. Similar behavior was found for the other detector (\#6), but so far only for small major radius plasmas $\left(R_{\mathrm{o}}=2.25 \mathrm{~m}\right)$.

In Fig. 4.21 is shown the normalized flux (flux/neutron emission) to the midplane detector versus the injected neutral beam power. The detector was maintained at $\mathrm{R}=340 \mathrm{~cm}$, well inside the $\mathrm{RF}$ limiters radius and consequently in the so-called "first- 


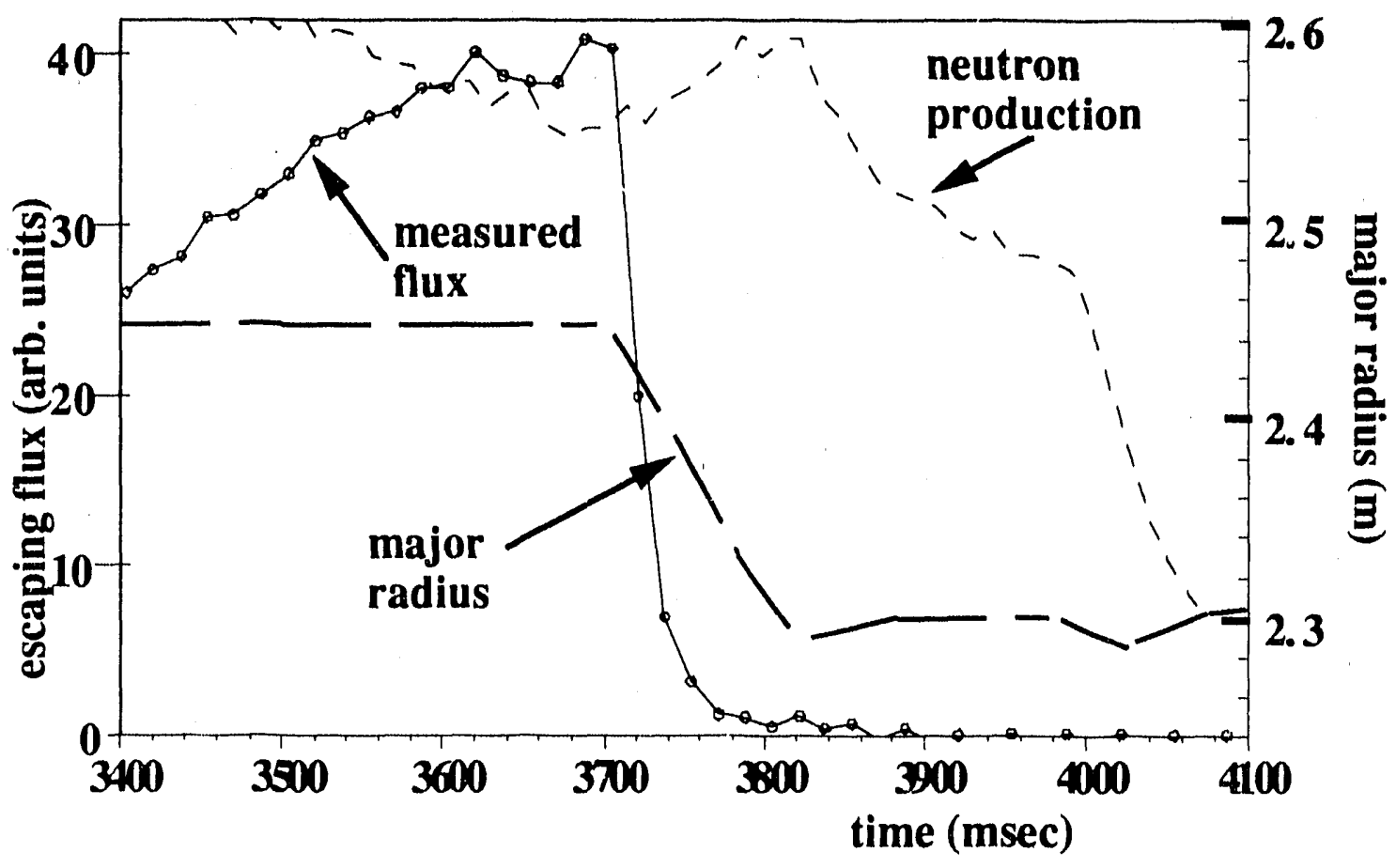

Figure 4.20: Time dependence of the escaping flux during a plasma compression measured by the midplane detector behind the RF limiters. The compression started at $3.6 \mathrm{sec}$ and was completed at $3.7 \mathrm{sec}$. The plasma was moved from a major radius of $2.45 \mathrm{~m}$ to $2.30 \mathrm{~m}$. 


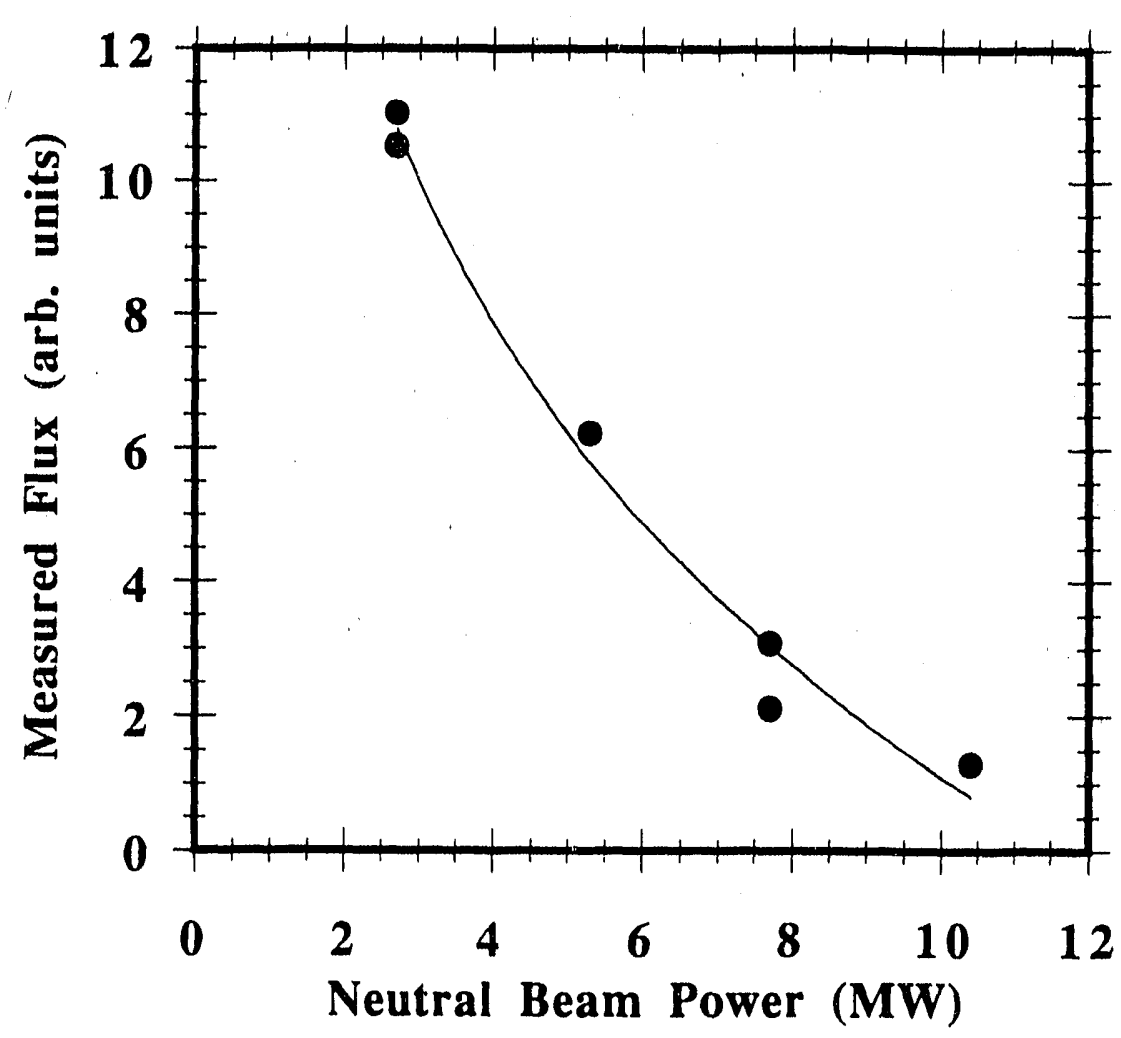

Figure 4.21: Neutral beam power dependence of the normalized flux to the midplane detector $\left(R_{o}=2.25 \mathrm{~m}, I_{p}=1.15 \mathrm{MA}\right.$ and $\left.B_{T}=5 \mathrm{~T}\right)$.

orbit confined" region. Calculations made by TRANSP showed that the current profile was unchanged during the scan and that the source profile did not change sufficiently to explain such a large drop in flux.

Naturally the big question here is where do the particles go? Are they lost to a different region of the wall? Are they confined in orbits near the center of the discharge (e.g. passing particles)? We have some indications of where they are ending up but we do not have a clear picture of the physical mechanism. For example, in Fig. 4.22, we show the time dependence of two discharges from the above beam power scan. The one at the left has only $2.7 \mathrm{MW}$ of NBI, whereas on the right $10 \mathrm{MW}$ were injected. During the steady-state period of the discharge (between $\sim 3.2-3.9 \mathrm{sec}$ ) the normalized level is 6 times less in the higher power than in the lower power discharge. However an additional flux appears after and at the very beginning of the beam injection. This behavior suggests a possible storage of particles in a different region of the plasma or in a different class of orhits. This argument is reinforced by the 
peculiar "blips" observed at the beginning and in the middle of the NBI. At those moments the beam ions pressure was smaller and losses tended to revert to the level shown in the top case.

We observed at rnany occasions, especially at high beam power, delayed losses of C'FPs up to 250 msec after the termination of the neutral beam injection. At that time the fusion production is already orders of magnitude lower and cannot explain such a big escaping flux. However, by looking at the gyroradius distribution we observed that these particles are partially slowed down. In Fig. 4.23 we show the gyroradius distribution for the same discharge $\left(I_{p}=1.4 \mathrm{MA}, B_{T}=4 \mathrm{~T}\right.$ and $R_{o}=2.60 \mathrm{~m}$ ) but at different times $(\sim \cdots 25 \mathrm{msec}, \sim 140 \mathrm{msec}$ and $\sim 175 \mathrm{msec}$ after the end of beam injection). On the last "frame" the peak energy is only $40 \%$ of the birth energy. This seems to indicate that previously stored particles are no longer in a confined region after the beams are shut, off but are detected only at the midplane.

At this moment no satisfactory explanation has been found for this behavior. One possible mechanism would be the transformation of some fraction of the trapped particles onto passing orbits (e.g. which would explain the early phase of the bottom case in Fig. 4.22), and vice-versa at the end of the discharge. Otherwise a simple change of regime from diffusing to non-diffusing may not be sufficient to explain the large difference. On the other hand the change from trapped to passing orbits would kcep particles much closer to the center of the discharge (the orbit shift of a passing particle scales like $\Delta \sim q \rho$, smaller than for trapped particles which scales like $\lrcorner \sim 2 q \rho / \sqrt{\epsilon})$. By being confined closer to the center there would be particles less detectable by the midplane probe near the outer wall. The dynamics of such a process is extremely complex and is time-dependent, beyond the scope of the present work. The situation is more complex than for a relatively simple case of plasma compression because in this case we are not really moving the plasma but essentially changing the value of $\beta$ in the discharge and consequently the equilibrium.

Such a dependence on beam power and the presence of additional losses after the NBI have been found in other conditions (different current and different aspect ratio). Fortunately, most, if not all of the dedicated scans were done at low and constant beam power (to avoid MHD activity). The results obtained by the bottom detector $\# 6$ was also found to be anomalous at small aspect ratio $\left(R_{\mathrm{o}}=2.25 \mathrm{~m}\right)$ and a special model will be needed for those special discharges. 

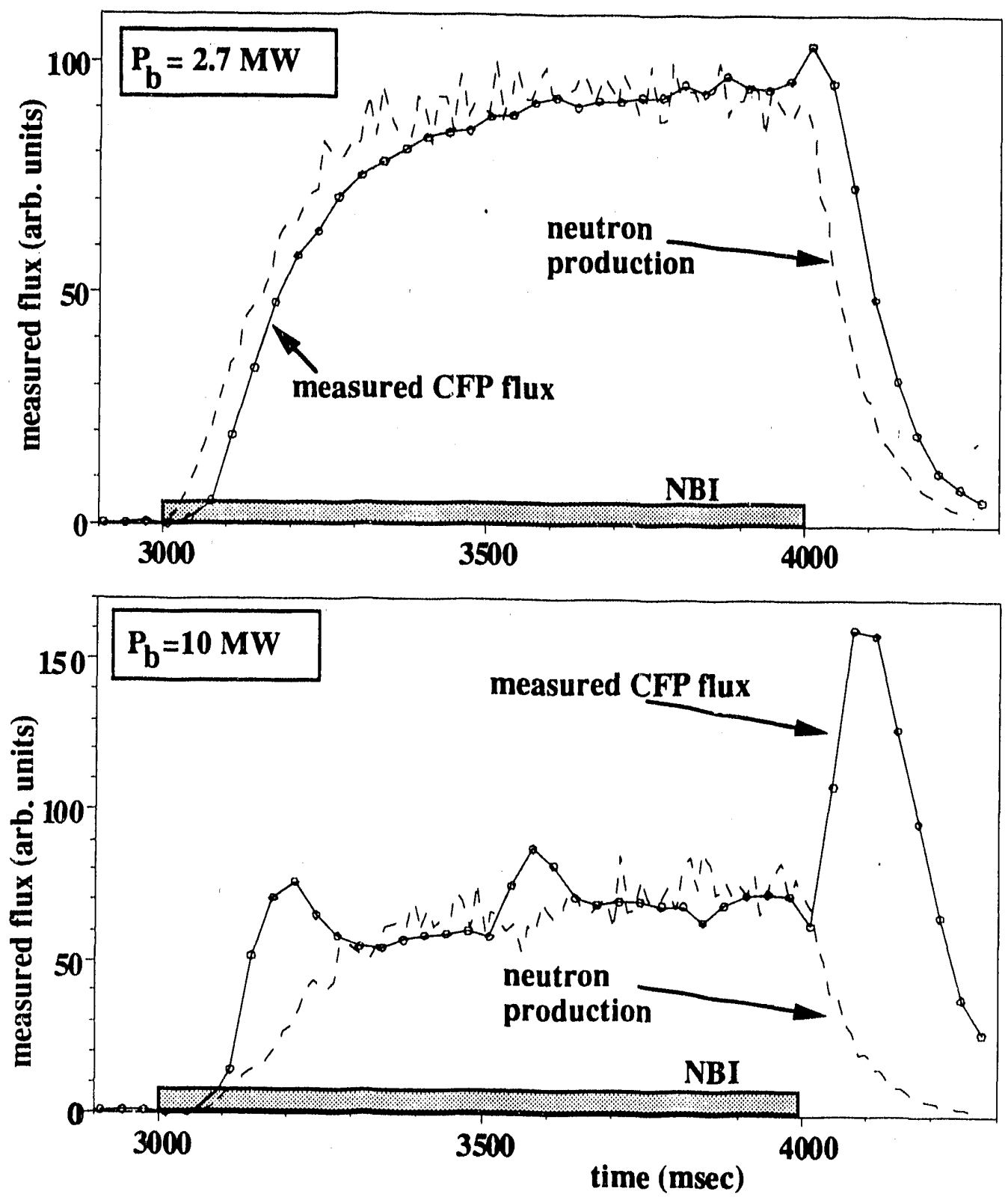

Figure 4.22: Time dependence of the CFP flin detected with the midplane probe with small amount of beam power (2.7 $\mathrm{MW}$, top) and larger amount (10 $\mathrm{MW}$, bottom). Also plotted is the neutron emission time dependence. The blip which occurs at approximately 3.5 seconds in the bottom plot is due to a brief bearn fault. 


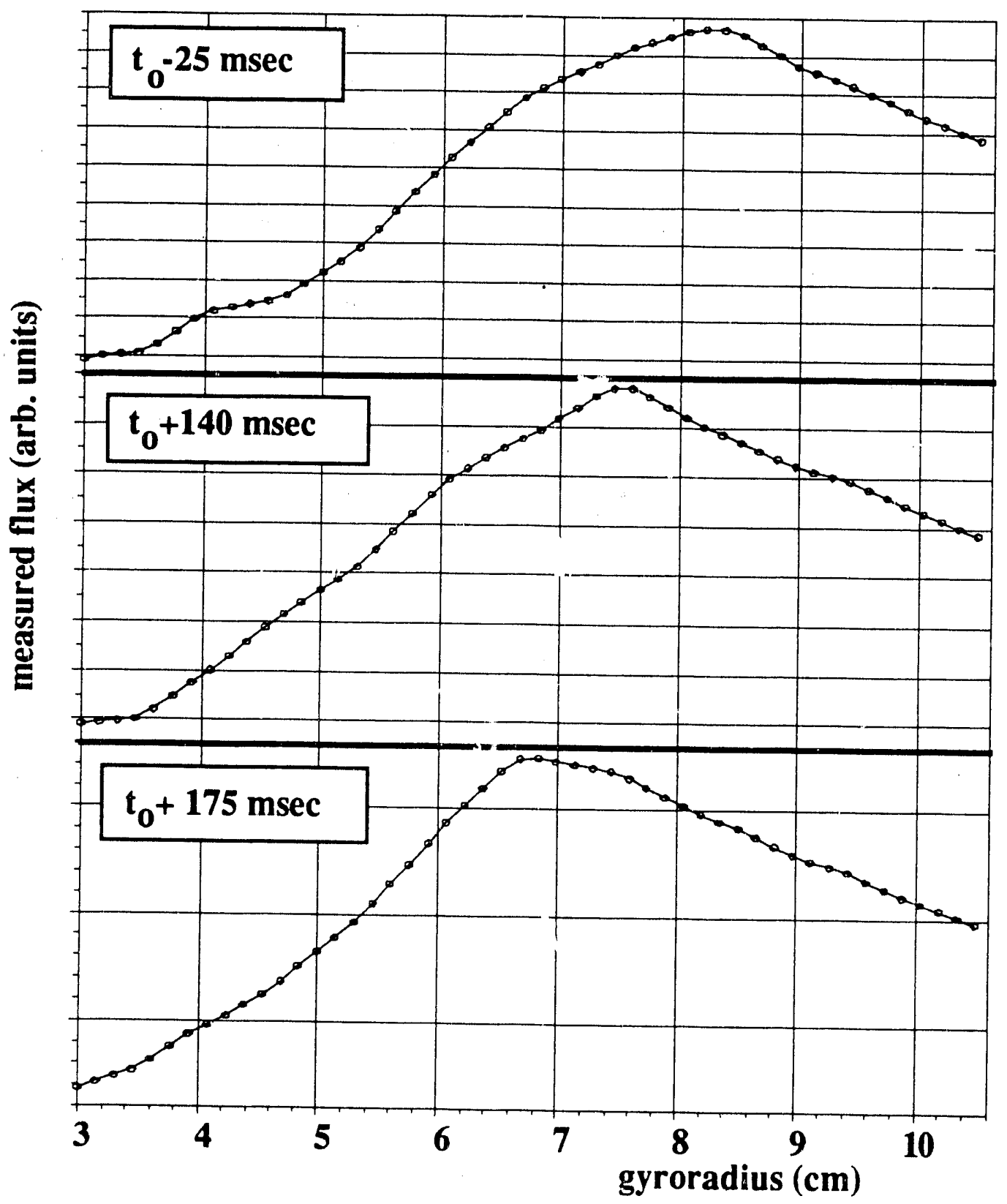

Figure 4.23: Gyroradius distribution of escaping flux after beam injection at relatively high beam power $\left(I_{p}=1.4 M A, B_{T}=4 T\right.$ and $\left.R_{o}=2.60 \mathrm{~m}\right)$. The three frames were taken at $\sim-25 \mathrm{msec} \sim 140 \mathrm{msec}$ and $\sim 175 \mathrm{msec}$ after the end of beam injection. 


\section{Chapter 5}

\section{Direct measurements of diffusion}

The experimental results described in the previous chapter were generally aimed at looking at the amplitude of CFP losses to the wall. The emphasis was put on determining, for example, the existence of the stochastic threshold ( $\varepsilon_{s}$, see Eq. 2.8) which is one of the key aspects of TF stochastic ripple losses. In this chapter, we will focus on the other aspect, which is the diffusion rate ( $\Delta r$, see Eq. 2.5).

In an ideal world, determining a diffusion coefficient would be trivial if one had measurements of the CFP density for every point in the tokamak. By solving the density continuity equation one could then infer the diffusion rate. In order to obtain such measurement, one would need to sample everywhere the population of C'FPs by using, for example, a non-intrusive probe (or by using a diagnostic like Thomson scattering, if the densities were sufficiently large, as expected in D-T operations). In these experiments the detector has finite dimensions, perturbs the local density of particles, and is restricted to a region near the outer midplane. However, since the detector is mounted on a movable probe and since its dimensions are relatively small, one can still expect to obtain some information about CFP populations but self-consistent models must be introduced.

The technique that we used for interring the diffusion rate of CFPs has been employed previously for diffusion studies of runaway electrons. ${ }^{128}$ Basically, we insert an obstacle (for the moment unspecified), which would block particles. The obstacle thus creates "behind" itself a shadowed region. By bringing the aperture in the shadowed region we can look at the perturbed CFP populations. In the absence of diffusion, the shadowed region would be well delimited (e.g. by having sharp transition boundaries, with a decay length roughly equal to the gyroradius) and also easy to 
explain by simple geometry argument (as in the clear-cut shadow of an object under the sun, for example). In the case where diffusion is present, particles will eventually fill the shadowed region. They would "sneak" behind the obstacle.

Practically, we have several means at our disposition to do this experiment. First, since the banana widths of CFPs are very large (30-50 cm, and even larger at low current), there exists a region outside the plasma, between the plasma and the wall (the vacuum region) where CFPs are present in large number and are confined on their first orbit (i.e. their first poloidal transit). In that region there are 3 obstacles that we can use to shadow the aperture (which leads to the scintillator). The first obstacle is the detector itself: a cylinder, $6.35 \mathrm{~cm}$ in diameter, and approximately 25 cm long and located $\sim 35.6 \mathrm{~cm}$ below the midplane. The key feature of the probe as an obstacle lies in the position of the aperture (where particles are in fact detected and whrch is approximately $2 \mathrm{~mm}^{2}$ in arca) which is $3 \mathrm{~cm}$ away from the probe tip. We will discuss further this important point below. In this case these first $3 \mathrm{~cm}$ of the probe tip self shadows the detector's aperture.

The second obstacie that we used is composed of the two RF limiters. Compared to the probe, they are quite large and are located $117^{\circ}$ and $171^{\circ}$ away in the countergoing direction from the detector (between bays $\mathbf{K}$ and $\mathbf{L}$, and between bays $\mathbf{N}$ and $O)$. The limiters have a relatively flat surface, with a toroidal radius curvature of $155 \mathrm{~cm}$ directed outwards, and are $50 \mathrm{~cm}$ wide, longer than the translation length $\left(=2 \pi v_{\|} / \Omega \approx 20 \mathrm{~cm}\right)$ of a CFP over a gyroperiod. They extend poloidally from the inner bumper limiter from the top to the bottom on the outer wall $\left( \pm 120^{\circ}\right)$, with a minor radius of $99 \mathrm{~cm}$, almost concentric $\left(R_{R F}=260.6 \mathrm{~cm}\right)$ with the vacuum vessel first wall $\left(R_{V V}=265 \mathrm{~cm}\right)$.

Finally, the third obstacle is a movable Langmuir probe, ${ }^{98,129,130}$ similar to the detector in its external dimensions, that we simply used as a "dummy" obstacle. The probe is located above the midplane $(36.8 \mathrm{~cm}$ above, compared to the detector located at $35.6 \mathrm{~cm}$ below) and the head is made of a cylinder $5 \mathrm{~cm}$ in diameter and approximately $20 \mathrm{~cm}$ long. It is installed at a different toroidal location, in TFT'R Bay A, toroidally $72^{\circ}$ in the co-going direction. Although the second probe is capable of a fast motion $(\approx 13 \mathrm{~cm} / \mathrm{sec}$ ), the results reported here were obtained by changing the obstacle position in between shots only. This approach simplified the interpretation of the results since some of the changes due to the presence of the obstacle could be due also to possible changes in profiles (current and source) during a shot. 


\subsection{Preliminary observations}

Before getting in the details of the physics involved in the experiments it is worthwhile to examine the experimental results of the different shadowing experiments and understand the conditions in which they were obtained. Since the step size is a strong function of the radial and poloidal locations of the bounce point (see Eq. 2.5) we must restrict our experimental results to a narrow band in pitch angle and in gyroradius.

The action of the RF limiters and of the detector's self-shadowing is studied together by using a single experimental setup. As we mentioned in Chapter 3, the detector can be moved approximately $23 \mathrm{~cm}$ inside the first wall of TFTR. The first $9 \mathrm{~cm}$ are directly behind the radius of the RF limiters where the aperture would be mainly shadowed by the limiters. In the remaining $14 \mathrm{~cm}$ the aperture is inside the radius of the RF limiters where the aperture would be shadowed by the detector only.

In Fig. 5.1 we show the measured flux as a function of the aperture radial position in the vicinity of the RF limiters only $\left(I_{p}=1.6 \mathrm{MA}, B_{T}=5 \mathrm{~T}\right.$ and $\left.R_{o}=2.45 \mathrm{~m}\right)$. Note that as the aperture is moved further outward in major radius, the $\mathrm{RF}$ limiters block a larger number of particles and so the detected flux of particles decreases substantially. In this case we look at particles with $60^{\circ}$ in pitch angle.

We repeated this experiment with different plasma conditions, mostly by piggybacking on other experiments. We found that the radial decay in measured flux changed somewhat with plasma conditions, especially with plasma current and aspect ratio. We compare the flux radial fall-off for two different currents (1.6 and 1.15 MA) in Fig. 5.2. Such behavior is certainly not surprising since the CFP orbits are a big factor in this experiment and we know that orbits are strongly affected by current and aspect ratio (see Fig. 5.7 and Eq. 4.7). The variation in the fall-off is expected to vary also with pitch angle (only in the diffusive case) since the step size depends on the bounce point location. However, we have very few complete scans which prevented a complete scan in different diffusion rates.

We will concentrate on the aperture radial scans on which we have a spatially well-resolved measurements. In other cases the radial spacing between data points is too large to permit a meaningful comparison with the numerical calculations. We also scans where the signal to noise (mainly neutron background) ratio is not large enough (that occurs mainly when the aperture is located very near the outer wall). The most complete scan was performed at $I_{p}=1.6 \mathrm{MA}, R_{o}=2.45 \mathrm{~m}$ and $B_{T}=5 \mathrm{~T}$ and is shown 


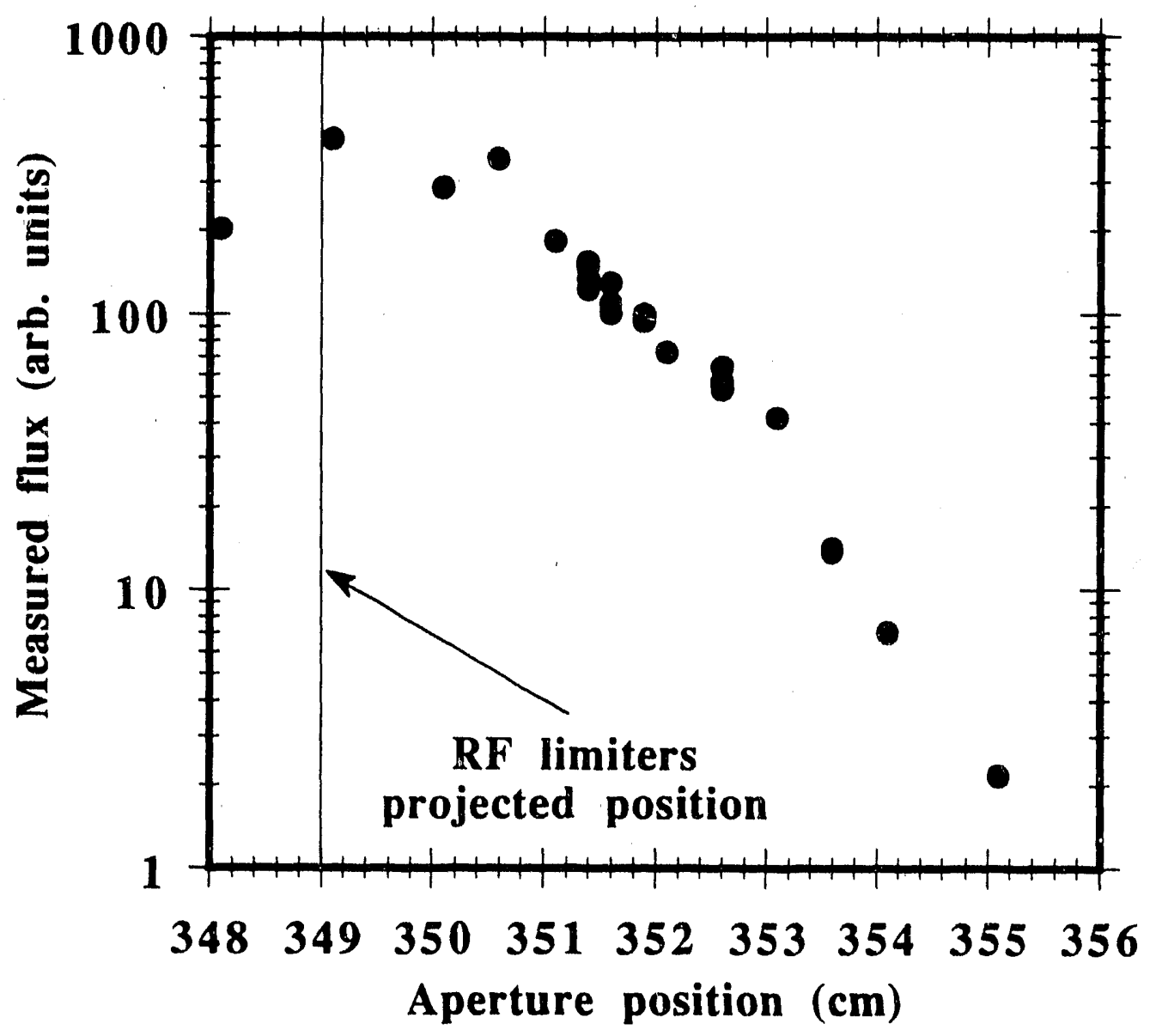

Figure 5.1: Aperture radial scan near the outer wall showing the transition from first-orbit confined particles to lost particles. The transition occurs approximately when the aperture passes in front of the RF limiters (at $\left.R_{o} \approx 349 \mathrm{~cm}\right) .\left(I_{p}=1.6 \mathrm{MA}\right.$, $B_{T}=5 T$ and $R_{\mathrm{o}}=2.45 \mathrm{~m}$ )

in Fig. 5.1 (see also Table C.5). The aperture was moved on successive discharges from its completely retracted position (wall) to $\approx 5 \mathrm{~cm}$ inside the local major radius of the RF limiters (the major radius of the limiters at the poloidal location of the probe), which is approximately $16 \mathrm{~cm}$ beyond the plasma edge. We see that the flux starts to drop at the major radius where an orbit leads to the RF limiters radius at the midplane $(\theta=0)$.

In Fig. 5.3 we show a similar scan in which the full extension of the probe has been used ( $I_{p}=1.15 \mathrm{MA}, B_{T}=5 \mathrm{~T}$ and $R_{o}=2.45 \mathrm{~m}$, see Table C.6). Note that once the 


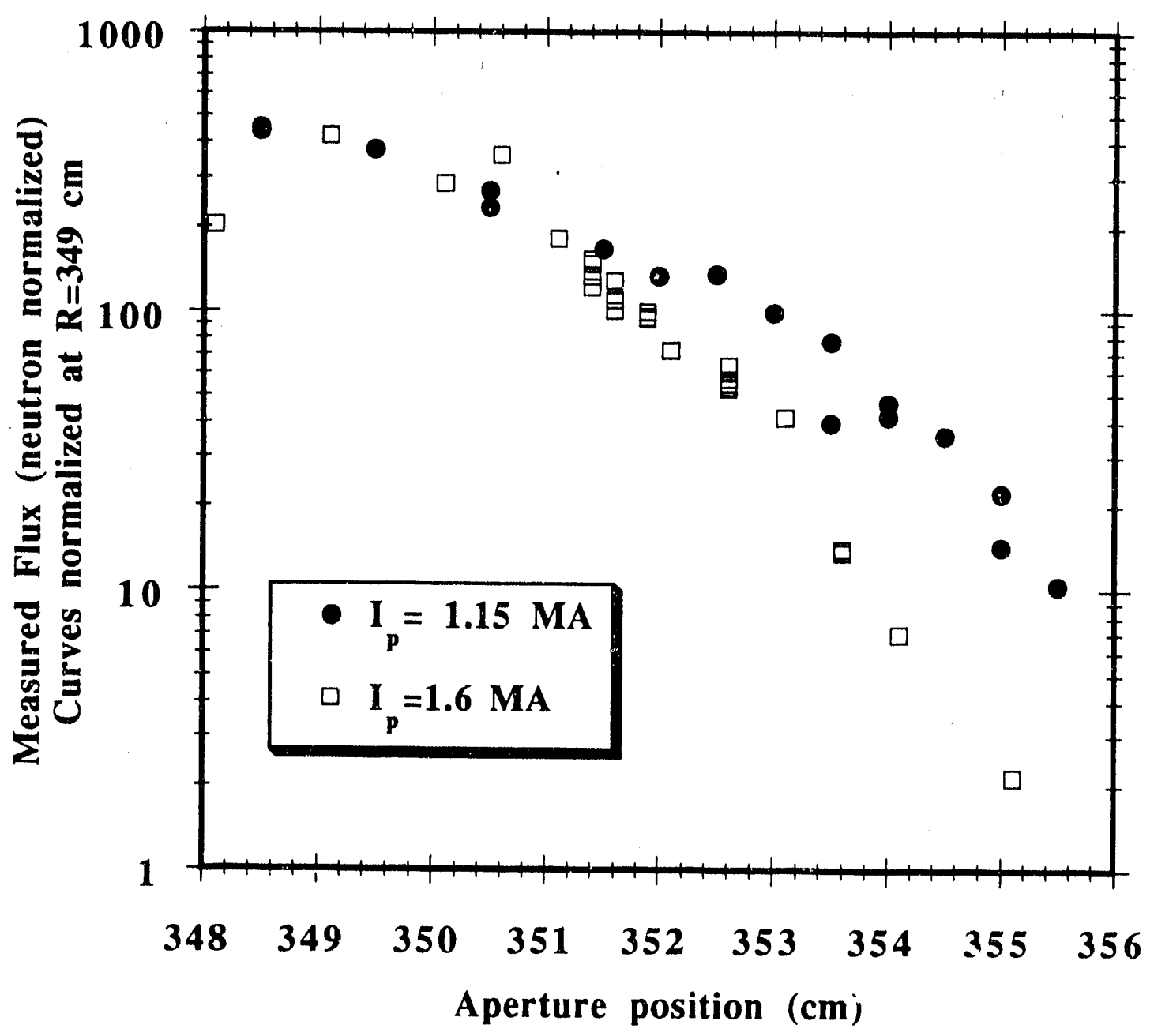

Figure 5.2: Comparisons of the radial decay in measured flux for two plasma currents (๑: $1.15 \mathrm{MA}, \square: 1.6 \mathrm{MA}$ ). Diffusion rates and banana widths are larger in the $1.15 \mathrm{MA}$ case explaining the different radial behavior. 


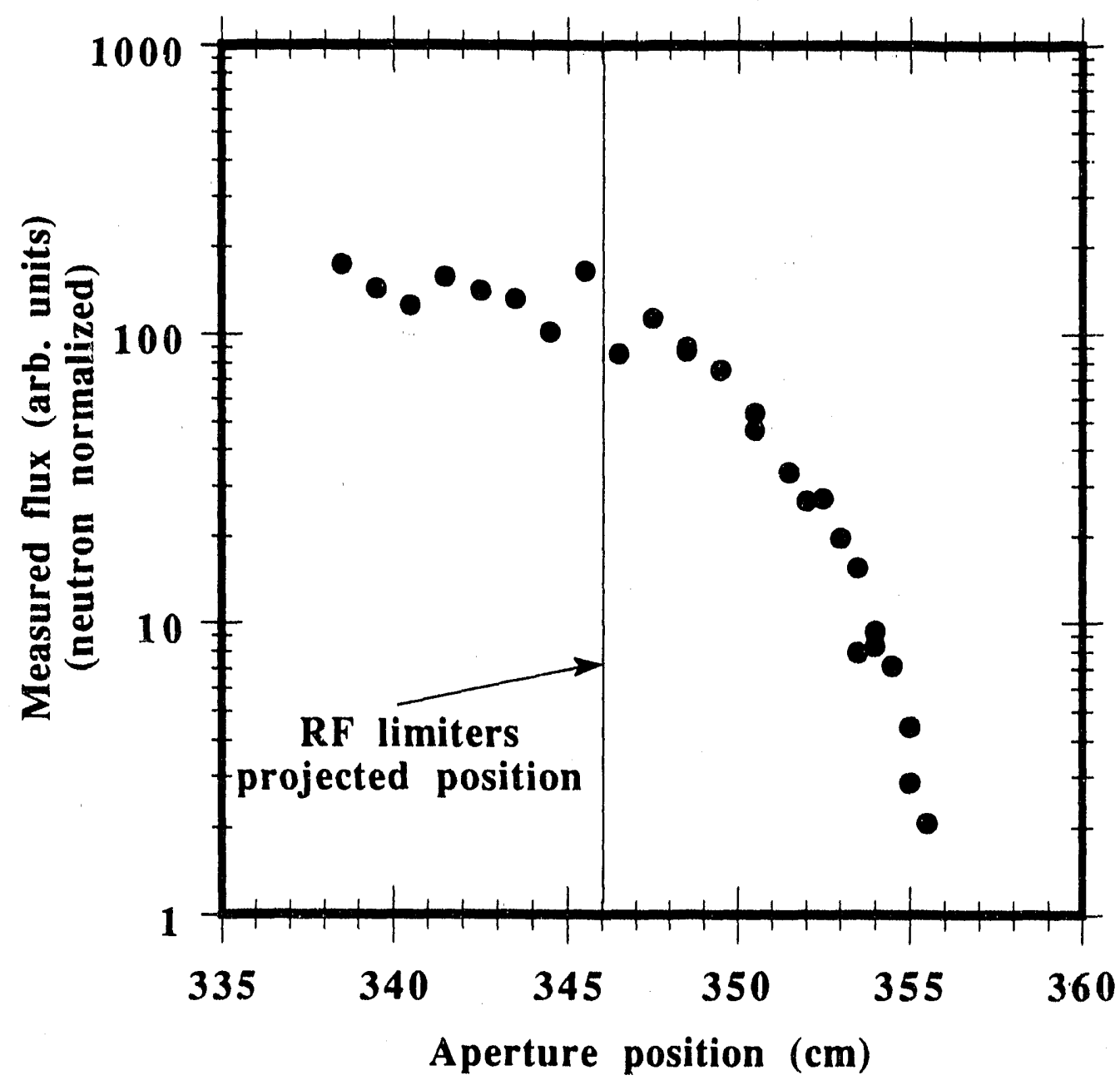

Figure 5.3: Complete aperture radial scan which starts behind the RF limiters up to full extension of the probe. $\left(I_{p}=1.15 \mathrm{MA}, B_{T}=5 \mathrm{~T}\right.$ and $\left.R_{o}=2.45 \mathrm{~m}\right)$

apert ure cleared the region shadowed by the RF limiters (on the left of the graph), the flux remains relatively constant. In this region, the flux is limited by self-shadowing from the detector. Here, the pitch angle is also restricted to be approximately $60^{\circ}$.

Finally, in Fig. 5.4 we show the results of the shadowing experiment done with the second movable probe in which the detector's aperture remained at $R_{\mathrm{o}}=347 \mathrm{~cm}$. At this location the effects of the RF limiters are small and so the shadowing action 
is due to the second probe and the detector. In this case the experimental points correspond to a pitch angle of $55^{\circ}$. The conditions chosen for this experiment were chosen to maximize ripple diffusion and to leave sufficient room between the plasma and the first wall for the probes to maneuver safely. Referring to Fig. 4.4 we see that a $I_{p}=1.4 \mathrm{MA}, B_{T}=5 \mathrm{~T}, R_{o}=2.45 \mathrm{~m}$ discharge would be suitable. Under these conditions the ripple value at the outer midplane edge is approximately $0.3 \%$. Clcibally we calculate that the CFP fraction lost would be approximately $3 \%$ (or $15 \%$ of the trapped particle population).

On successive discharges, the obstacle was moved radially inward from outside the first wall (approximately $20 \mathrm{~cm}$ beyond the aperture), to approximately $20 \mathrm{~cm}$ in front of the aperture. We observed a very large and relatively sharp decrease of the escaping flux as a function of the obstacle position. When the obstacle approached the aperture radial location we observed that the flux decreased dramatically, and with the full extension of the obstacle it dropped to only $12 \%$ of the original level. The experiment was performed over two different run days, almost three months apart (•: July' $6^{\text {th }}$, 1]: Septcmber '22 $2^{\text {nd }}$, see Table C.4). The different symbols used correspond to the different run days. We immediately notice the very good reproducibility of the experimental curve.

\subsection{Diffusion model}

The interaction of obstacles with the orbits of CFPs can be a very complex situation. The most challenging difficulties are the large helical orbits (gyroradius of 5-10 cns) and large drifts from the CFP birth flux surfaces (with banana widths of 30-50 cm or more) which are comparable in dimensions with the obstacles. Fortunately, we can make some assumptions that will make the interpretation of the experimental results a more tractable problem. We will start with the detector itself, assuming no wher obstacles. Then we will discuss the shadowing effect with the RF limiters, and, finally study the case where another probe was inserted as an obstacle. For each case we develop a specific diffusion code which is slightly different in the way that CFP orbits are treated. When the obstacle is sufficiently small, compared, for example to a gyroradius (i.e. bay $\mathbf{A}$ probe), we can, to a certain degree, ignore the orbital effects of the CFPs. In other words, the CFPs do not drift significantly (or, simply, they follow the local field line in the vicinity of the object) over the diameter of the 


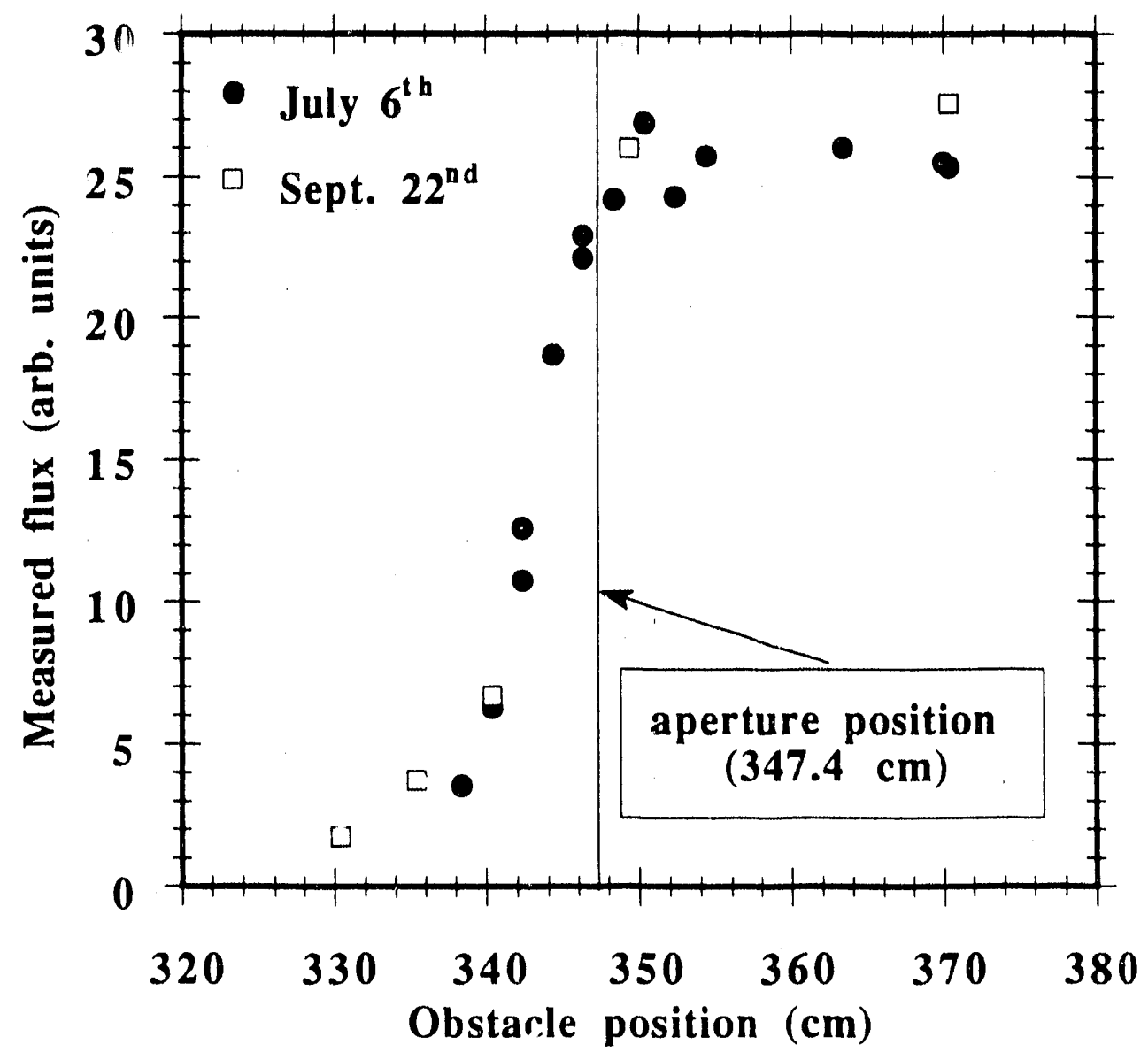

Figure 5.4: Experimental results of the shadouing experiment with a second small probe. The second probe (the obstacle) is moved radially from beyond the aperture (aperture located at $R=347.4 \mathrm{~cm}$ ), to in front of it. The different symbols correspond to different "run days. 
obstacle or the detector. In the case of the large obstacle (i.e. RF limiters) the CFPs drift significantly on the scale of the obstacle, both toroidally and poloidally. In this case, we must include some orbital effects that we will describe in Sect. 5.2.2.

The different models (with some supplementary details described in App. A.2) have some common traits that we will now describe. Since the quantity that we measure is the flux of particles at the aperture, we compute the expected flux by following particle orbits backwards in time; we start a particle at the aperture (with given pitch, gyro and toroidal angles). 'The particle is stopped when it either hits something (detector, second probe, wall or RF limiters) or when it goes back deeply in the plasma - and reaches the threshold boundary for example. For each poloidal transit before the final impact (or before the return decply into the plasma), we increment a counter by the magnitude of the CFP source profile for particles born on that orbit. However, since we restricted our analysis to cases near the outer wall, and since we found that the probability of impact on an obstacle (including the detector) was much larger than the probability of returning deeply in the plasma, variations in the source profile were found to be negligible. Note also that even though a particle's bounce point can move in radially, its banana width is reduced accordingly (see Eq. 4.7) and so a change in source profile would be also reduced.

Consequently, in the case of the shadowing experiment with the RF limiters, we calculated the flux to the aperture as being simply the number of poloidal transits before any impact.

In the case where an obstacle is gradually brought in (i.e. second movable probe) we compared the fluxes using a scheme shown in Fig. 5.5 and we used source profile weighted calculations. In that case the flux to the aperture will be reduced since the particle could not go back in time as far as in the case without the obstacle. In the case illustrated at the top the obstacle is absent and the particle eventually hits the wall (for example). In the case illustrated at the bottom we added the presence of an obstacle and the particle is stopped before hitting the wall. In the case shown the particle makes $x$ poloidal transits instead of $y$ before impact. Consequently, the flux to the aperture (with the obstacle) would be approximately only $x / y$ times the flux previously detected (without the obstacle).

The error bars of the numerical simulation include also uncertainties arising from our specification of the boundary conditions. In anticipation of a more complete description of the different models it is worthwhile to outline the different cases with 


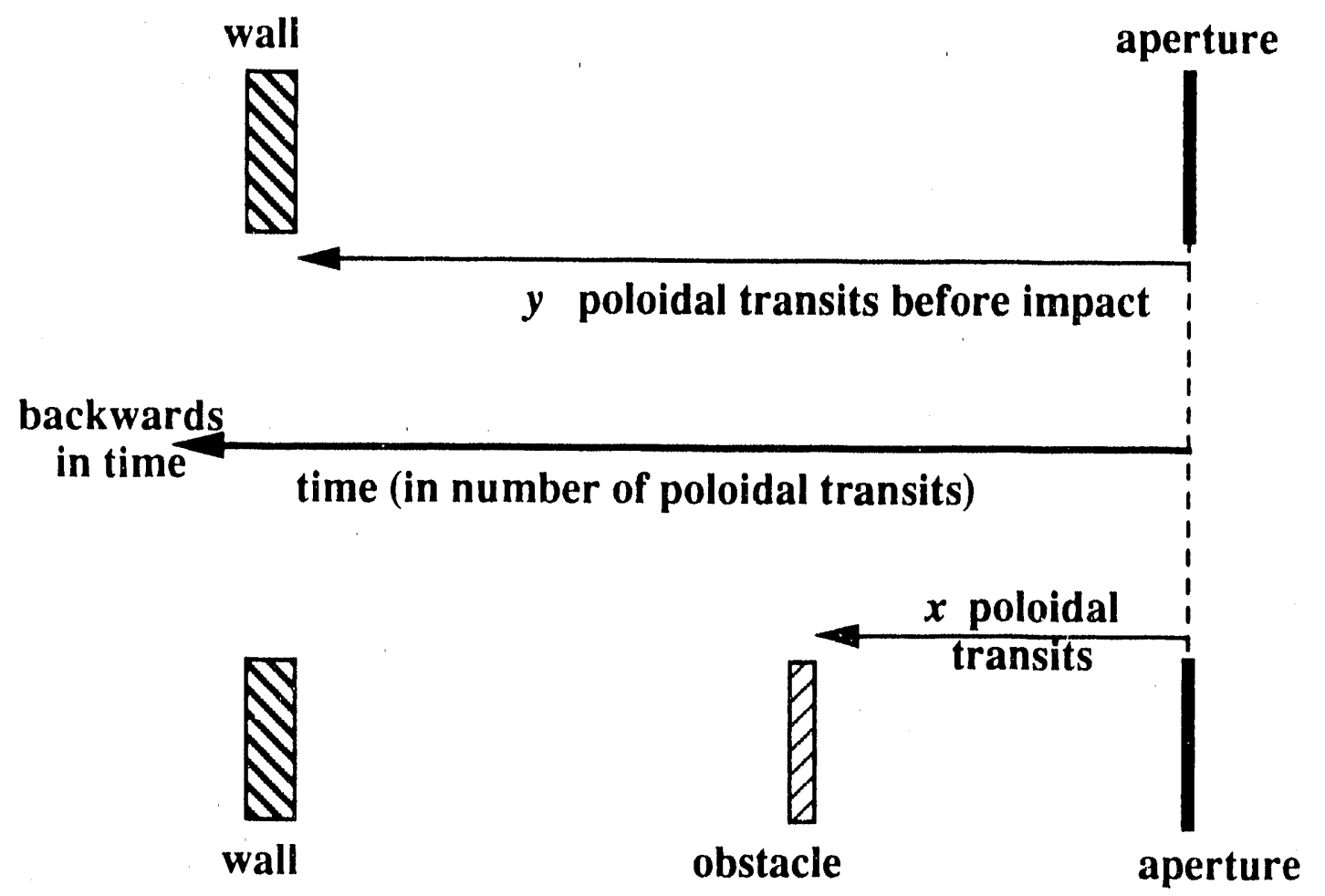

aperture

$\mathbb{8}$

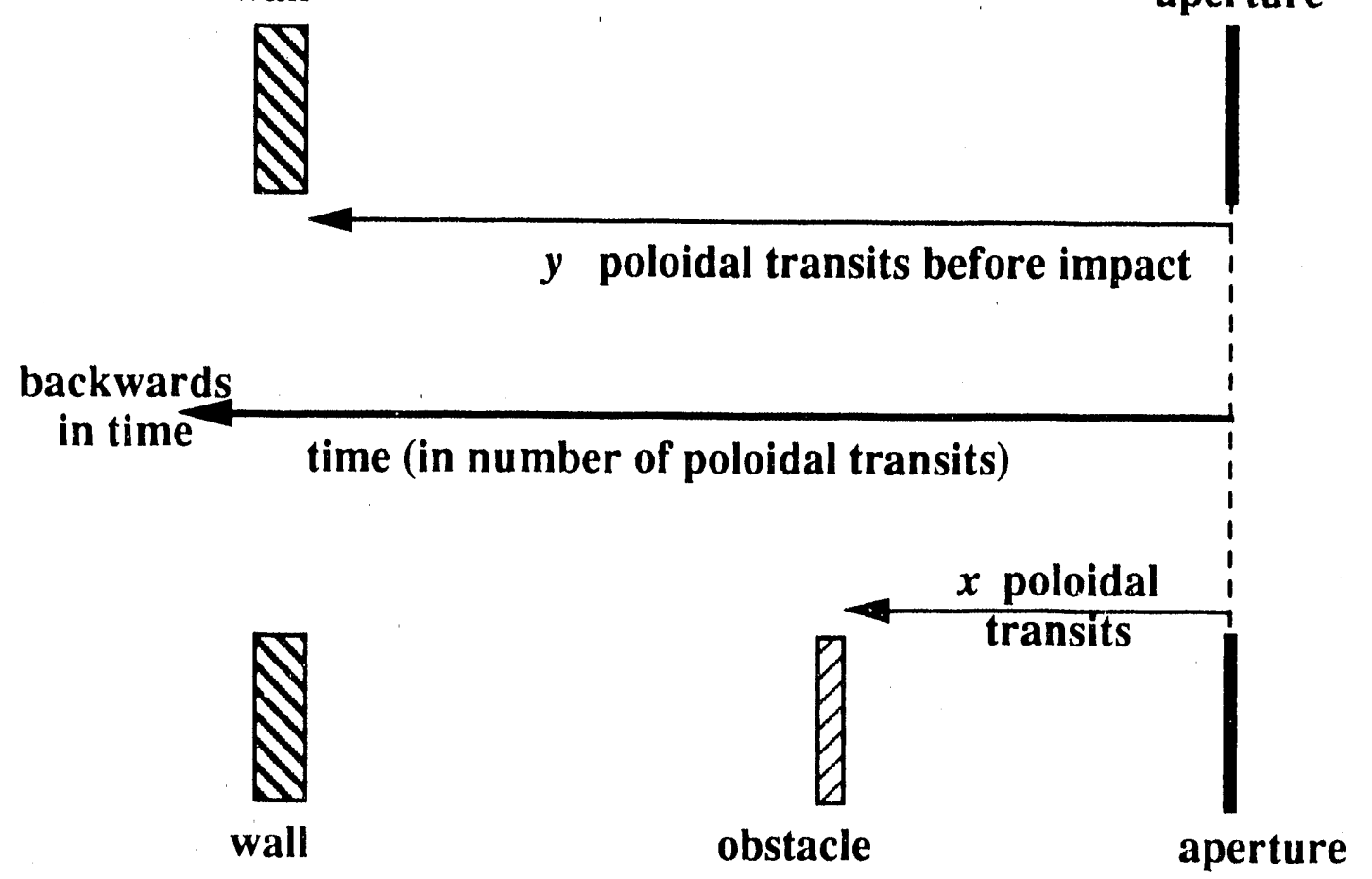

Figure 5.5: ('omparison between cases with (bottom) and without an obstacle (top) shouing the difference in flux to the aperture. In the example without the obstacle, the particle would impact on the uall. In the bottom case the flux would be reduced by a factor of $x / y$ from the top case. 
their key aspects:

\section{Self-Shadowing}

- Detector only obstacle (e.g. "fly catcher").

- Particles detected were confined on their first orbit.

- Small probability of particle impact on detector.

- Detector size $\approx$ g.'roradius.

- Orbit details negligible.

- Finite Larmor radius effects non negligible.

- Particle toroidal location random.

Large obstacle, RF limiters

- $\mathrm{KF}$ limiters dominant obstacle, larger than detector.

- Large toroidal and poloidal extensions.

- Guiding-center orbit important.

- Probability of hitting limiters $\gg$ hitting detector (or penetrating aperture),

- Self-shadowing of detector implicit in model (important when aperture inside RF limiters radius).

- Finite Larmor radius effects negligible.

\section{Small obstacle, Bay A probe}

- Obstacle dimensions $\sim$ detector dimensions.

- Similar location (obstacle and detector).

- Small probability of particle impact.

- Self-shadowing of detector implicit in model. 
- Orbit details negligible.

- Finite Larmor radius effects non negligible.

- Particle toroidal location random.

\subsubsection{Detector self-shadowing}

It is important to understand the effects of the detector's presence on its own detection efficiency. If we start a particle at the aperture, how many bounce times is there before it impacts onto the detector? This calculation represents consequently the maximum flux that can be detected. The aperture can not see particle orbits going backwards in time further than the time between two impacts. We can make estimates using simple considerations about the detector dimensions. These estimates will be corroborated in the next sectio as with the use of numerical calculations.

We show in Fig. 5.6 the toroidal cross-section of the tokamak (top view) taken at the elevation $(\mathrm{Z}=-35.6 \mathrm{~cm})$ of the aperture. In this case, we are dealing with the detector only, and the particles are not diffusing $(\mathrm{D}=0)$. Consequently, for each poloidal transit, when the particle is at the same elevation than the aperture/detector, the particle will be somewhere in the toroidal band of $2 \rho$ in width shown shaded. In the outer region of the vacuum vessel (i.e. near the detector) this description is good for both passing and trapped particles. Assuming that the toroidal angle is random (which is legitimate when we consider many particles at an irrational $q$ surfacc, the toroidal precession, and also small variations in pitch angle or in gy'roradius), the probability of self-shadowing would be simply the ratio of the toroidally projected area of the detector over the total surface of the band. In other words for each poloidal transit the detector will catch a fraction of particles equivalent to $A_{\text {tip }} / A_{\text {orbit }}$. This estimate is legitimate since the detector is relatively small; the CFP does not drift sufficiently from its flux surface in the vicinity of the detector. We find, from the ratio of the areas that it takes between 500 and 1000 poloidal transits (or simply, twice as many bounce times) before a particle started from the aperture of the detector would later impact on the probe head. In other words, this would mean that in the absence of diffusion, the aperture can only look back in time approximately 1000 to 2000 bounce times (approximately $10 \mu \mathrm{sec}$ for the $1 \mathrm{MeV}$ triton) or, about 10 to 20 msec (for the triton), before it hits the detector. 


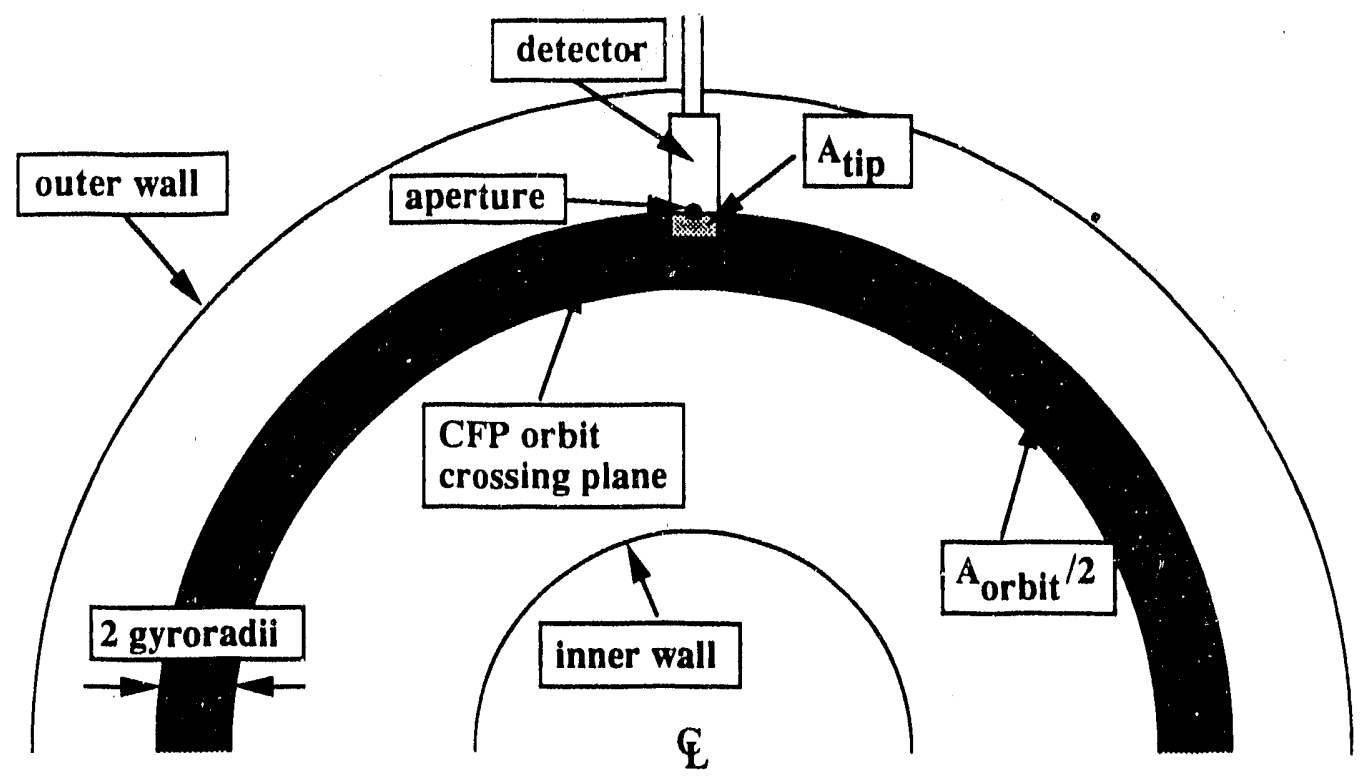

Figure 5.6: Toroidal cross-section of the tokamak shouing the detector and the region where the orbit intersects the toroidal plane at the elevation $(Z=-35.6 \mathrm{~cm})$ of the aperture. The probability of impact per poloidal transit would be approximately the ratio of the projected areas $A_{\text {tip }} / A_{\text {orbit }}$. 
A nother important direct consequence of this estimate is that for measuring diffusion rates by this technique, particles must diffuse at least $3 \mathrm{~cm}$ (distance between the tip and the aperture) over the 1000 to 2000 bounce times between successive impacts. Particles are detected only at the aperture and if particles are diffusing too slowly they would be scraped-off by the probe head tip before reaching the aperture. For a triton (see above), this means that the diffusion rate must be larger than $(3 \mathrm{~cm})^{2} / 10$ msec $\sim 0.1 \mathrm{~m}^{2} / \mathrm{sec}$, which is low enough for the study of TF ripple diffusion (by at least an order of magnitude).

However, the experimental verification of the self-shadowing effect cannot be done directly at the moment. Since the perturbation created by the detector itself can not be varied easily (e.g. the distance between the probe tip and the aperture), and since we do not have a sufficiently accurate absolute calibration, we must rely on additional perturbations (bring additional obstacles) in order to study the diffusion process.

\subsubsection{Diffusion in the presence of a large obstacle}

In the case of a large obstacle (i.e. with the presence of RF limiters at the outer wall) we must take in account some details (for more details see App. A.2.2) of the actual orbit followed by the particle near the outer wall. The limiters have a broad poloidal extension (from $\theta \simeq-120^{\circ}$ to $120^{\circ}$ ), so trapped particles, on banana-shaped orbits, can impact the limiters at many poloidal locations. Near the wall, the particles also travel extensively in the toroidal direction, increasing their chances of impact. Here we move the aperture/detector not the obstacle. It is clear that when the aperture is positioned well inside the radius of the RF limiters, the flux would be limited by the detector self-shadowing which we discussed in the preceding section. On the other hand, when the aperture is outside the radius of the RF limiters, the flux would be largely reduced by the limiters iue to their relatively large size.

The algorithm that we used starts particles at the aperture. From there, the bounce point is found using a modeled banana orbit derived from calculations made by ORBIT. Diffusion is then applied to the radial position of the particle bounc. point. We take Eq. 2.5 as the expression for the step size. The banana orbit is then followed (using the modeled orbit) until the particle either hits an obstacle (RF limiters or the detector) or reaches the upper bounce point. The cycle starts again from the bottom hounce point and continues until an impact orcurs. There is also a small probability 
for the particle to return to the center of the plasma, those particles are simply stopped when they are found to be near the stochastic threshold boundary; typically near half the minor radius. The flux to the aperture is taken as being proportional to the number of poloidal transits (up to a few thousand) for a particle before the impact on any object (detector or limiters) or before reaching the threshold boundary. We also found that the numerical results were relatively insensitive to changes in boundary conditions (i.e. with possible variations in the threshold boundary location) and they are included in the error bars for the numerical curves in Fig. 5.8. However, the model becomes increasingly unreliable when the aperture is located well inside the radius of the RF limiters where the modeled orbits and plasma are no longer valid.

One immediate result of these calculations is related to the question of detector self-shadowing discussed in Sect. 5.2.1. In the absence of diffusion, when the aperture is well inside the radius of the RF limiters we should recover the result obtained in the previous section. We found in this case that the particle would take between 800 and 1000 bounce times ( $1 / 2$ poloidal transit) before impact the detector in good agreemsint with the previous calculations made with the simplified diffusion code for the small obstacle case.

Comparison of the numerical calculations with the experimental results are shown in Fig. 5.8. We normalized all curves to the $R_{\text {aperture }}=349 \mathrm{~cm}$ point where the limiters should play a minimal role. The first observation that we make from the experimental data is naturally the absence of a sharp transition across the RF limiters boundary, expected from the no diffusion case. In the absence of diffusion, particles are either blocked or not by the limiters. The reasons for a sharp fall-off (in the no diffusion case) are two-fold. First, the particles travel on a long distance toroidally near the outer wall $(\Delta \phi=q \Delta \theta)$ and are likely to hit the limiters when they are outside their minor radius. Second, the $\mathrm{RF}$ limiters are wider than the translation length of a CFP (distance along a field line over a gyroperiod) eliminating any gyroradius effect. However, this sharp fall-off would only occur if we consider particles at a single pitch angle and energy. The radial location of this transition is a strong function of the banana width, which in turn, is a function of the particle pitch angle and energy. For that reason we only took the measured flux at $\simeq 60^{\circ}$, and between 7 and $8 \mathrm{~cm}$ in gyroradius (near $1 \mathrm{MeV}$ for the triton).

As a comparison with the experimental results, we included in Fig. 5.8 the numerical results obtained with the model described in Sect. 5.2.2. We observe from the 


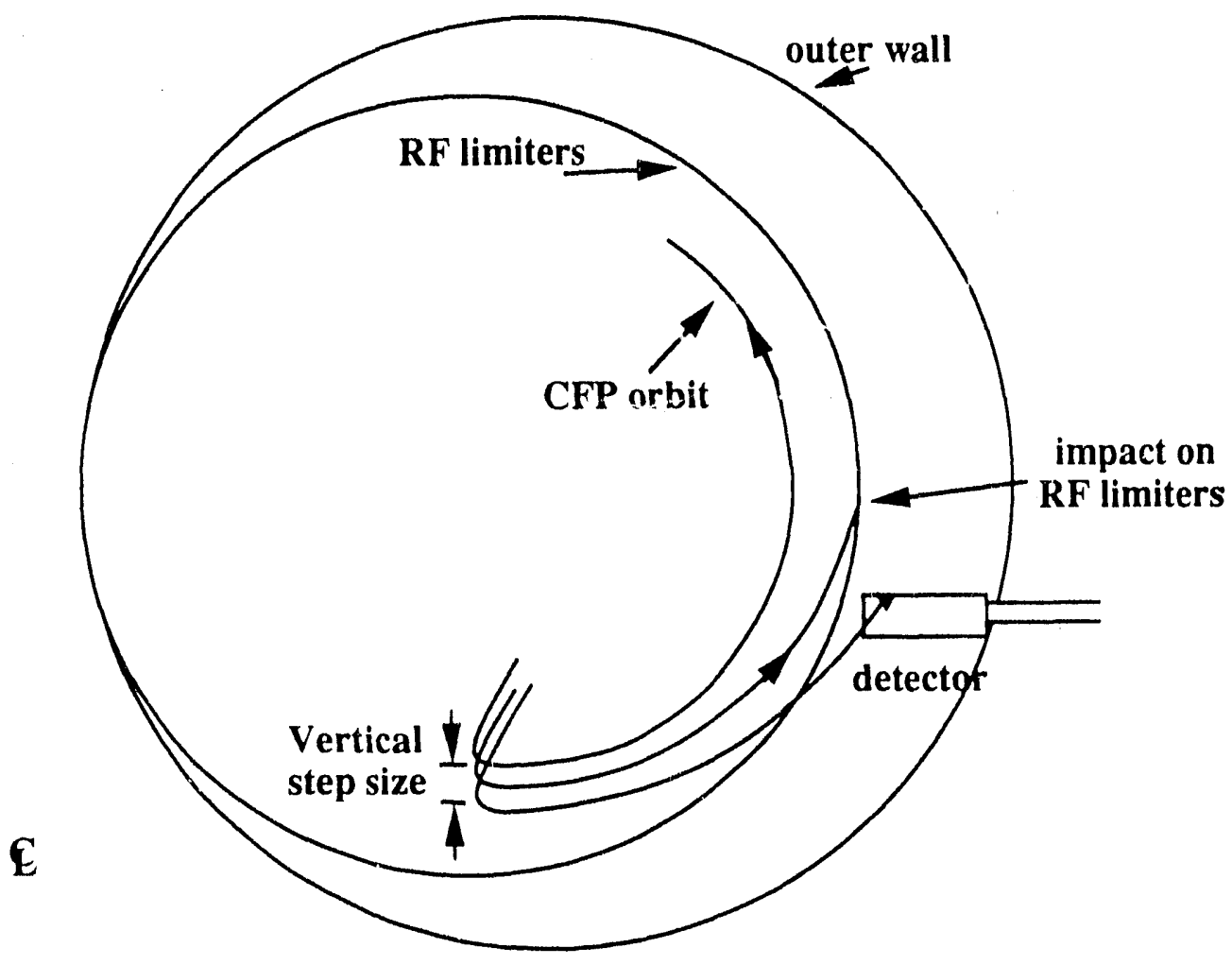

Figure 5.7: Physical picture of the shadouing experiment with the RF limiters. With the aperture moving across the $R F$ limiters radius some of the diffusing particles will be blocked by the limiters. The radial fall-off rate is then a function of the diffusion rate of the CFPs. 
experimental results, that the flux of CFPs that reaches the aperture behind the RF limiters is much larger than what we would expect without diffusion $(\Delta r=0, \square)$. In the presence of diffusion we used for the step size the expression found in Eq. 2.5. We vary the step size amplitude using a global factor keeping the same parametric dependence. As we increase the diffusion rate (step size), proportionally more particles will slip behind the limiters (compared to the $R=349 \mathrm{~cm}$ level). The best fit was found with $\Delta r=0.65 \pm 0.2 \mathrm{~cm}(\diamond)$ (at $R_{\text {aperture }}-349 \mathrm{~cm}$ ) which is within $15 \%$ of the theoretical expectations. If we increase the step size further (to $\Delta r \approx 3 \mathrm{~cm}, \triangle$ ), we cannot replicate the large drop in flux. In fact, in the extreme limit $(\Delta r \rightarrow \infty$, dashed line) the limiters would have no effect on the measured flux.

\subsubsection{Diffusion in the presence of a small obstacle}

Comparatively to the large obstacle case discussed above, the obstacle is relatively small. Consequently, the reduction in flux was found to be important but not as drastic as in the previous case (see Fig. 5.4). The similarities between the detector and the obstacle will introduce simplifications in the calculations that we will describe below. The first consideration concerns the position of the aperture itself. By positioning the aperture inside the RF limiters radius, not only do we reduce the problems related to boundary conditions (walls, limiters) but, as we saw in Fig. 5.1, we also increase dramatically the detectable flux. Since both probes are in a similar poloidal location and are of similar dimensions which are small compared to the CFP orbit, we can neglect the details of the orbits. In other words the particle do not drift significantly in the vicinity of both probes. As a first approximation, the detector can be seen as a two dimensional object with radial and toroidal extensions but none poloidally. We simply project the obstacle and the detector into a toroidal plane as we did in Fig. 5.6. As we mentioned before, we start the particle at the aperture and follow it backwards in time using its guiding-center location. For each poloidal transit $\left(=2 \tau_{b}\right)$, the particle would return at the detector poloidal location (which is not spelled out explicitly), where the radial location is based on a random walk diffusion process and the toroidal location is determined randomly.

Before discussing the numerical results and compare with the experimental data shown in Fig. 5.4, we can make basic estimates using area considerations similar to the one made in the previous section. Let us take the case with no diffusion, thus 


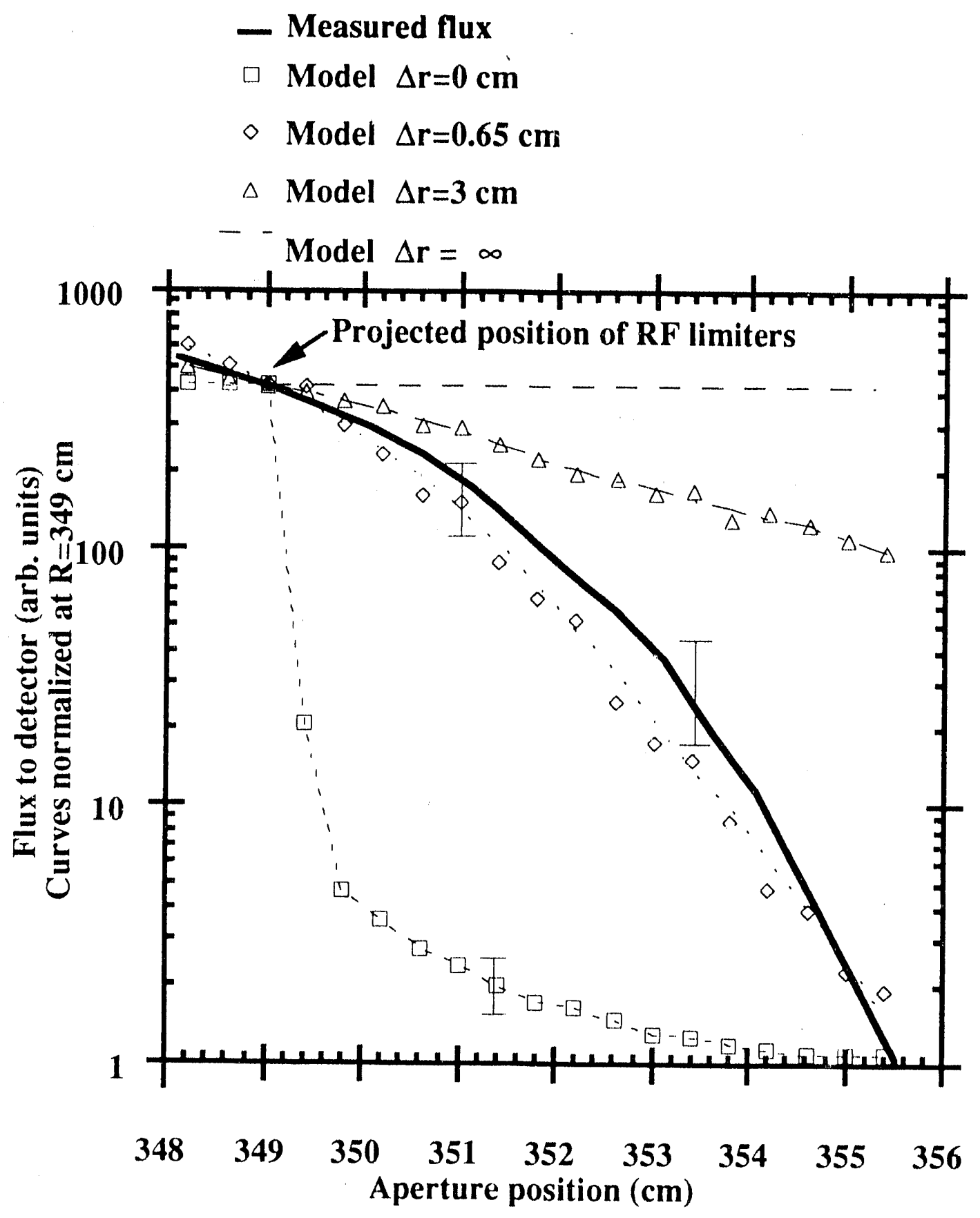

Figure 5.8: Comparison of the experimental results (at $\chi \approx 60^{\circ}$ ) of the aperture radial scan with the theoretical model including TF ripple diffusion. Also shown is the no-diffusion case with the expected sharp transition at the RF limiters radius. 
considering the detection of confined particles. In Fig. 5.9 we project a portion of the particle orbit into a toroidal cross-section showing the detector and the obstacle (same basic approach than in the self-shadowing case). As in Fig. 5.6, the orbit is itself represented by a band with a width of $2 \rho$ (assumed constant), divided in three sections of equal probability for the particle location (we chose to divide the band in three since the detector was covering one third already). The band stops at the aperture and represents the range of orbits that can be detected. Note that the detector tip protrudes $3 \mathrm{~cm}$ into that band (the distance between the aperture and the tip of the detector), which is coincidentally, approximately half the particle gyroradius. The relative probability of hitting either the detector or the obstacle is thus proportional to their respective area present in the orbits band. In Fig. 5.6 we have three cases for the situation in which the obstacle has been moved inwards. In the first case, when the detector and the obstacle are equally distant and rovering the first zone of the shaded band, the flux to the detector should be $A_{\text {detector }} /\left(A_{\text {otstacle }}+A_{\text {detector }}\right) \approx 0.54$ times the flux without the obstacle (here $A_{i}$ is the projected area of the detector or the obstacle and $A_{\text {obstacle }} / A_{\text {detector }} \simeq 5 / 6$ ). At that point, the obstacle has moved $3 \mathrm{~cm}$ past the detector aperture. In the second case, the obstacle moved over the second zone. The flux to the aperture is then proportional to $A_{\text {detector }} /\left(2 . A_{\text {obstacle }}+A_{\text {detector }}\right) \approx$ 0.37 times the flux without the obstacle. In the final case the flux to the aperture is further reduced, this time it would be $A_{\text {detector }} /\left(3 A_{\text {obstacle }}+A_{\text {detector }}\right) \approx 0.28$ times the flux without the obstacle. This level would be the lowest possible in the case with no diffusion. If one moves the obstacle further in, the obstacle does not block more particles present in the band. It would simply block particles which would not reach the aperture anyway. The situation would be different though in the case when diffusion is present, as we will see below.

Numerically, we use a simple random walk diffusion process. For every iteration, the radial position is determined through the position of the guiding center and a random gyroangle (for more details see App. A.2.1). The basic iteration scheme can be summarized as follows:

$$
\begin{aligned}
r_{g c, i+1} & =r_{g c, i}+\Delta r\left(r_{g c, i}, \phi_{i}\right) \\
r_{p a r t i c l e, i+1} & =r_{g c, i+1}+\rho \cos \left(\alpha_{i+1}\right)
\end{aligned}
$$

where $r_{g c, i}$ is the radial position of the guiding center, $r_{p a r t i c l e, i+1}$ is the particle radial position, $\rho$ is the gyroradius. $\alpha_{i+1}$ and $\phi_{i}$ are respectively the gyro and toroidal angles, 


\section{Obstacle flush with detector}

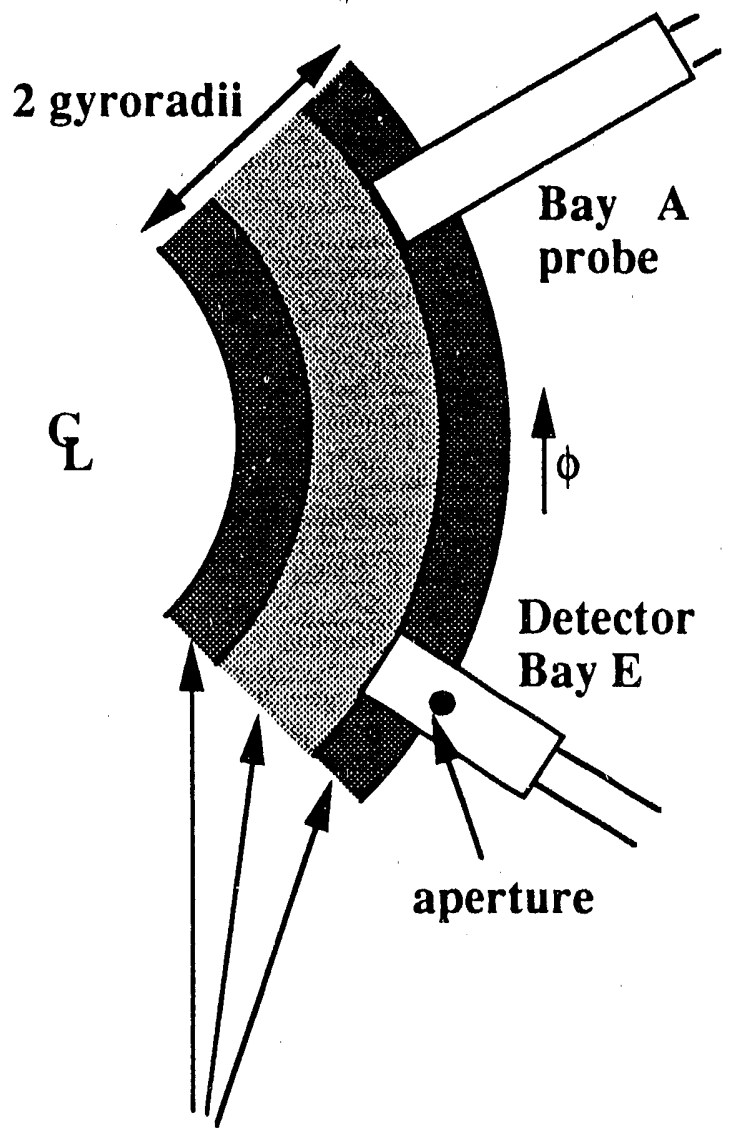

Obstacle partially extended

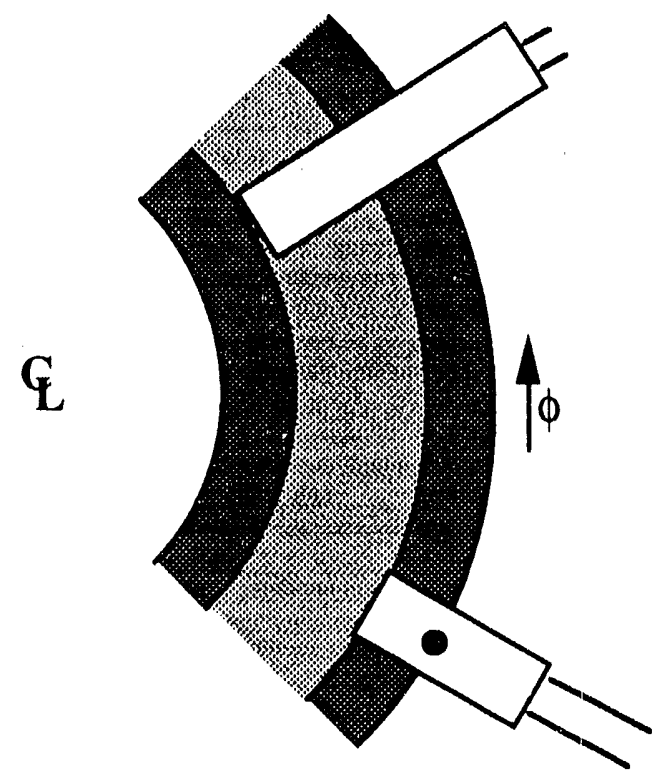

\section{Obstacle fully extended}

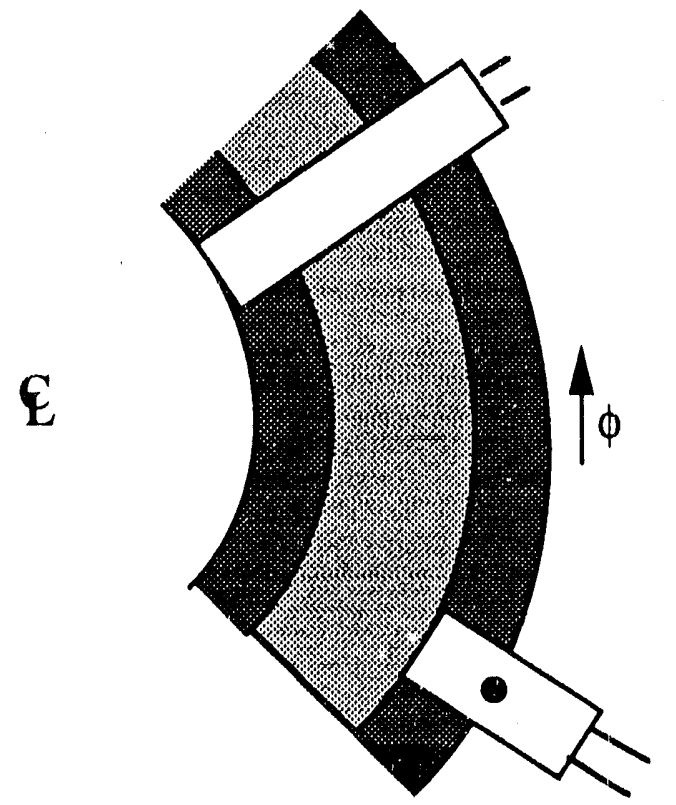

Figure 5.9: Toroidal projection of the shadowing experiment with the Bay A probe (a langmuir probe, located above the rridplane) used as an obstacle. The obstacle is shown here at three different locations cutting progressively the flux of CFPs to the detector's aperture. The three different zones in the orbit band represent regions of equally probable presence of a CFP. 
which are determined randomly. Let us emphasize the fact that the random variable here is the gyroangle, not the radial position itself. This effect is crucial because when we project the orbit on the plane of the aperture, we find that the particle has a larger probability to be at the extremes in $r$. In other words, the radial distribution of the particle's location probability is not uniform but scales as $\sin ^{-1}(r / \rho)$. Finally, $\Delta r\left(r_{g c, i}, \phi_{i}\right)$ is the step size corresponding to Eq. 2.5 and is used as the free parameter, the fit with the experimental results is done through this variable only.

In order to make the model a bit more realistic, we also introduce some corrections for the detector finite poloidal extension, by considering the range of gyroangles for which the particle would be within the finite diameter of the obstacle or detector. That correction effectively increases slightly the probability of hitting the objects for every pass. The toroidal extensions of the objects are also corrected for the pitch angle of the particle; the diameter of the probes is projected onto the toroidal plane by dividing the probe area by the cosine of the pitch angle (typically $55^{\circ}$ or $60^{\circ}$ ). However, these corrections will only modify slightly the area based estimates of the shadowing effect obtained above, these estimates were found numerically to be increased by approximately $15 \%$.

Without a sufficiently accurate absolute calibration we model the relative falloff for different diffusion rates. We are thus comparing cases with and without the obstacle, according to a scheme shown in Fig. 5.5. All curves are normalized at $R_{\text {obstacle }}=370 \mathrm{~cm}$, corresponding to the obstacle being completely out. In Fig. 5.10 we show now the experimental results compared to our model with different diffusive step sizes. The experimental vertical error bars are indicative of the shot to shot variations whereas the horizontal ones correspond to the uncertainty in the detector/obstacle position.

We will discuss first the case with no diffusion, as shown in Fig. 5.10 ( $\square$ ). Here, particles are confined and stay fixed to the same flux surface. In this case we retrieve the approximate levels estimated above using simple arguments involving the geometry and dimensions of the detector and the obstacle. If we increase the diffusion rate only slightly (i.e. for $\Delta r \leqslant 0.05 \mathrm{~cm}$ ) the obstacle would not block more particles than in the no diffusion case. The reason is that in this case particles would not diffuse fast enough to reach the aperture. In other words, at low diffusion rate, self-shadowing is important.

If we increase the diffusion rate, in the case shown to $\Delta r:=0.3 \mathrm{~cm}(\diamond)$, the 


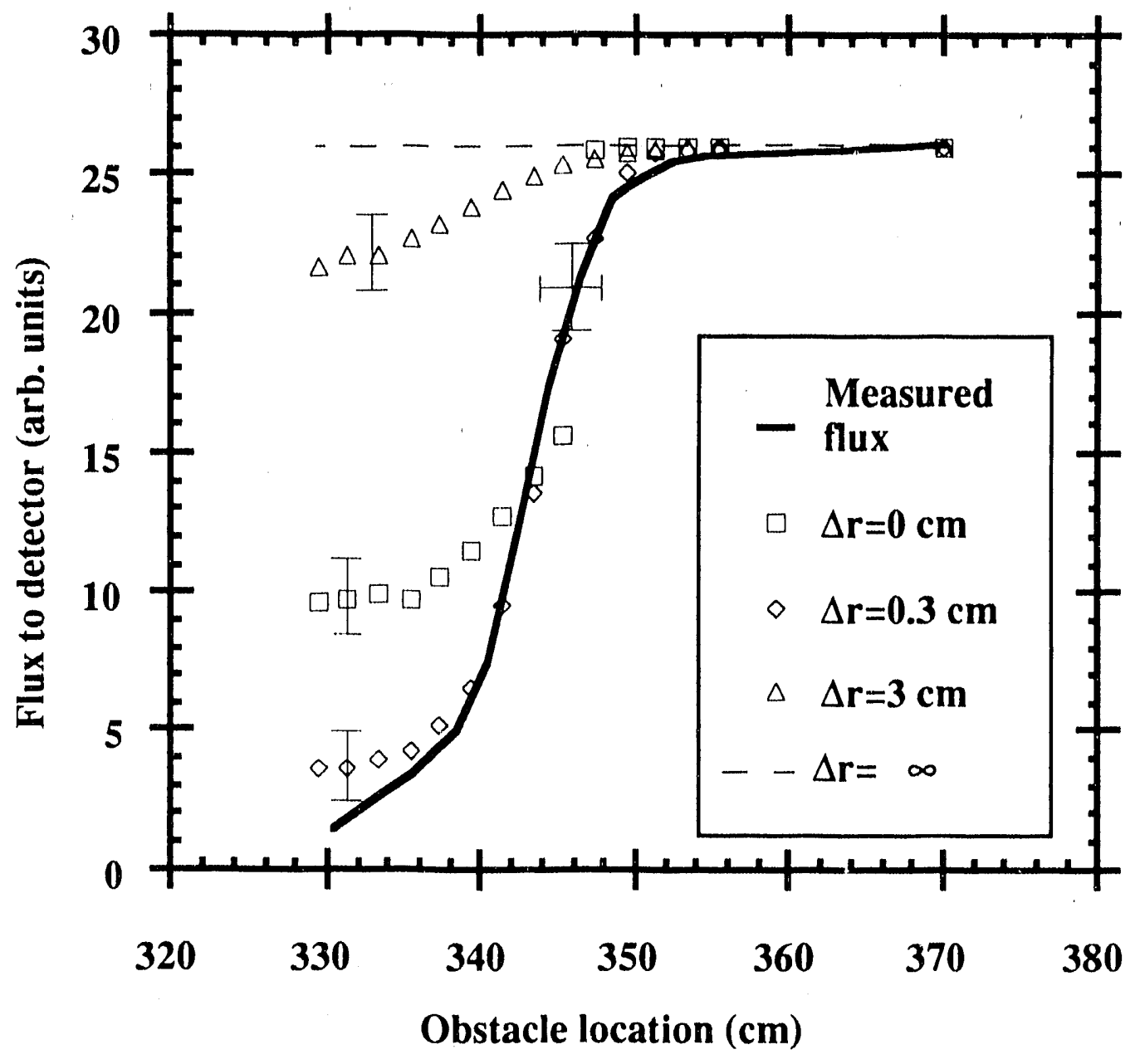

Figure 5.10: Measurements and calculations of the radial fall-off the escaping flux as a function of the obstacle position. The experimental results, shown by a continuous line are compared to numerical calculations involving different diffusive step sizes. All the calculated curves are normalized at the $R_{\text {obstacle }}=370 \mathrm{~cm}$ point. 
particles diffuse out much faster than in the previous case. However, the particles are still sufficiently slow so that they have a larger probability to hit the obstacle before reaching the aperture. In other words, they diffuse fast enough to avoid hitting the tip of the detector but still not enough to avoid being blocked by the obstacle, especially when this one is located very far inside the aperture (e.g. $\sim 15 \mathrm{~cm})$.

Finally, in we increase the rate again, to $\Delta r-3 \mathrm{~cm}(\triangle)$, the particles are diffusing too fast, the obstacle does not have the "chance" to efficiently block particles before reaching the aperture. We have to keep in mind that we are comparing cases with and without the obstacle. Naturally, if we compare the absolute flux (without the obstacle) between this case and the preceding one we would find that with very large diffusion rate very few particles would reach the aperture, they would simply reach the wall very quickly. In the previous case the detected flux (again without obstacle) would be optimum since particles would diffuse fast enough to avoid the probe tip but not too fast to pass by without being detected.

Notice that in the limiting case of $\Delta r \rightarrow \infty$ the curve would become a simple horizontal straight line (shown in the figure with a dashed line); the obstacle would not block any particle. That is what we would observe if the scintillator would detect particles lost on their first orbit. The pitch angle distribution would follow a firstorbit loss distribution, which we did not observe as we will see below. For example, the flux of particles to the bottom detector has been found, in this series of shots $\left(I_{p}=1.4 \mathrm{MA}, B_{T}=5 \mathrm{~T}, R_{0}=2.45 \mathrm{~m}\right)$, to be independent of the obstacle position, a partial indication that only first-orbit losses (analog to a very large step size) were reaching that detector.

Consequently, we have the very interesting case where we can, at least, put an upper and a lower bound on the diffusive step size. Both the lower bound, $\Delta r=0$, and the upper bound, $\Delta r=3 \mathrm{~cm}$, are unable to replicate the large decrease in flux at the obstacle full extension. However, the curve with a step size of $0.3 \mathrm{~cm}$ represents the best fit obtained with our model. By considering the uncertainty in the measurements and in the model, we can thus restrain the experimentally obtained step size to be between $0.2 \mathrm{~cm}$ and $0.4 \mathrm{~cm}$.

We can compare the obtained step size with the one expected theoretically by using Eq. 2.5 and the orbit code ORBIT. By retracing the orbit from the aperture to the the bounce point (which gives us $r_{b}$ and $\theta_{b}$ for evaluating $\Delta r$ ) we found that $\Delta r$ should be $0.3 \pm 0.1$ in very good agreement with the obtained result. The uncertainty 
in the theoretical value of the step size is mainly due to the uncertainty in $q(r)$.

The effects of a progressive shadowing with the obstacle are also visible in the pitch angle distribution. As we saw in the previous chapter, in the absence of diffusion the maximum flux comes at the fattest banana pitch angle, which corresponds to the orbit which comes the closest to the plasma center. When a diffusing process is present the distribution will exhibit the presence of an additional peak at higher pitch angle. In this case the detected flux is dominated by diffusing particles. However, the obstacle is more efficient in blocking diffusing particles than confined particles (D $=0)$ if it is placed sufficiently ahead of the aperture. That difference in "blocking efficiency" is visible in Fig. 5.10 where $\sim 63 \%$ of the flux is blocked if the particles are not diffusing and more than $80 \%$ if they are. Consequently, when the obstacle is positioned well in front of the aperture, we should see the pitch angle distribution change. In Fig. 5.11 we show the pitch angle distribution for two different obstacle positions. We see that, at the maximum obstacle extension, the distribution is largely shifted towards the pitch angle of the fattest banana, in this case located at $\chi \simeq 49^{\circ}$.

However, the pitch angle distribution does not change monotonically with the obstacle position. When we insert the obstacle further in, the peak of the distribution briefly moves to higher pitch angles (middle curve, in Fig. 5.11) and then moves down to lower pitch angles (i.e. the bottom case in Fig. 5.11). We believe that this behavior is a manifestation of different diffusion rates for different pitch angles. Recall that the step size is a strong function of the ripple amplitude (which depends on the minor radius and the poloidal angle) so diffusion rates will change with the bounce point location. For example, from the location the bounce point of different pitch angle particles (using the orbit code), we found that theoretically at $\chi \simeq 50^{\circ}$ the particle is out of the stochastic area $(\Delta r=0)$, at $\chi \simeq 55^{\circ} \Delta r=0.3 \mathrm{~cm}$, and at $\chi \simeq 60^{\circ}$ $\Delta r=0.7 \mathrm{~cm}$ (for a particle detected at $R_{\text {aperture }}=353 \mathrm{~cm}$ ). Particles with a small diffusion rate (around $55^{\circ}$ ) would be cut first (middle curve) then the ones with a larger diffusion rate (around $60^{\circ}$ ) leaving the first-orbit losses only (bottom curve). Unfortunately, the detector resolution in pitch angle is not sufficiently good for a complete deconvolution of the different diffusion rates. 

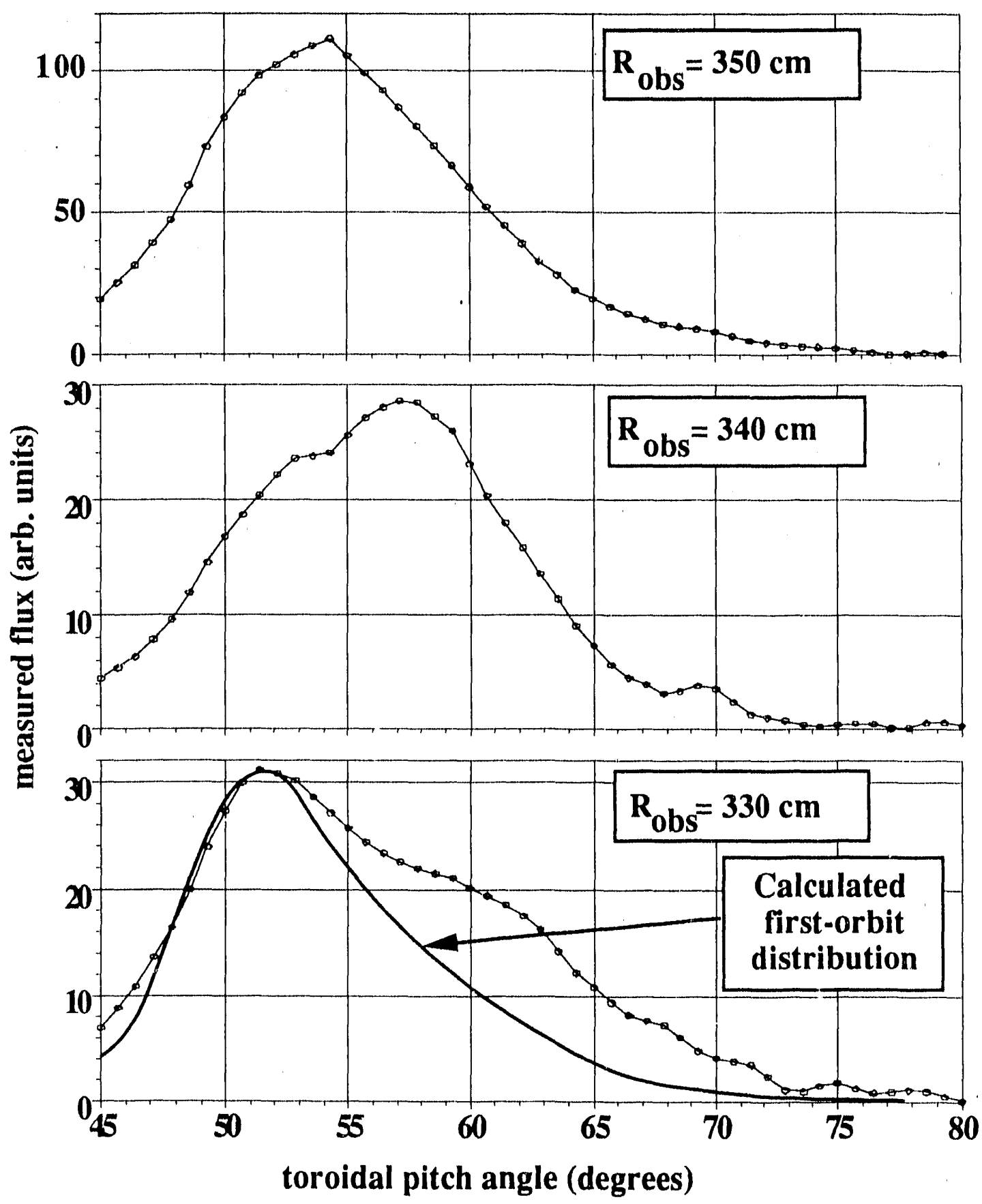

Figure 5.11: Pitch angle distributions obtained at different obstacle locations (on top, $R_{\text {otstacle }}=350 \mathrm{~cm}$, on the middle curve, $R_{\text {obstacle }}=340 \mathrm{~cm}$ ) and at the bottom $R_{\text {costucle }}=330 \mathrm{~cm}$. Diffusing particles were progressively blocked leaving only the first-orbit losses visible at lower pitch angle. Calculations from MAPLOS show the expected first-orbit loss distribution (bottom case). The gain used for the camera was increased by a factor of 3 from the rniddle case (top and middle are the same) to the bottom case (all with same neutron production). 


\section{Chapter 6}

\section{Other observed loss mechanisms}

On some occasions, especially when in the piggy-back mode, data has been acquired on phenomenon mot necessarily associated with TF ripple losses. In most cases, the experimental conditions were naturally not optimum for the study of CFP confinement. In fact, in some of these circumstances the parameters were precisely the ones that we tried to avoid (as in the experiments discussed in the previous 2 chapters) because of a possible combinations of loss mechanisms. For example, the confinement of CFPs was found to be modified during large scale MHD activity, typically at low toroidal field. Sawteeth were also found to affect moderately the loss of CFPs. We will describe some of the observations with some of their most striking features. But first, we will start with experiments performed by using short injections ( 20-50 msec) of neutral beams (beam blips).

\subsection{Neutral beam blips}

In order to study the confinement of beam ions, experiments were performed using short injections of fast neutrals and looking at the time decay of the neutron production. ${ }^{69,131}$ Experimental results were then compared with different diffusion models from which beam ion radial diffusion coefficients were inferred. Considering the fast time scale involved (beam blips), we used the set of photomultiplier tubes to look at the time evolution of the escaping flux. By doing so, we also lose sorne part of the spatial information carried in the complete image of the scintillator. Neutron production was naturally relatively small so the detector was mainly used in the "confined" region. One plasma condition was used ( $I_{p}=0.8 \mathrm{MA}, B_{T}=4 \mathrm{~T}$ and $R_{o}=2.42 \mathrm{~m}$ ) 
which by itself is not the optimum condition for the study of ripple diffusion (large first-orbit losses).

In spite of the limited scope of this study, we found an interesting phenomenon which could be used further in a future experiment. For example, we found that the time decay of these blips differ slightly depending on the inferred ion energy. In Fig. 6.1 we show the time dependence of the flux for two different gyroradii (at $\rho \sim$ 9 .m or $E \approx 3 \mathrm{Mel}$. and at $p=4 \mathrm{~cm}$ or $E \approx 0.6 \mathrm{MeV}$ for a proton) at approximately. the same pitch angle. We see immediately the differences between the two curves, especially at the end of the decay just before the next blip. Similar differences were found on single blip dincharges but were not as clearly visible as in the case shown here.

The simplest explanation to that phenomenon would be that some diffusing and partly slowed down particles reach the detector time-delayed. On the other hand, one would have to take in account the slowing down of the beam ions which are responsible for most of the fusion reactions. The Doppler shift would also decrease with their slowing down and cause some differences in the energy spectrum of the escaping CFPs. A systematic study would be necessary before concluding anyhting from these experiments, especially by doing a current scan and thus comparing different confinement regimes. A careful analysis of the gyroradius distribution is also necessary since the energy resolution of the detector (see Fig. 3.10) is only fair.

\subsection{MHD-induced losses}

Fast ion confinement has been found to be affected by . MHD activity, especially in presence of fishbone modes. ${ }^{132-138}$ CFPs were also found to be affected by large wherent MHD activity and several cases have been reported from the bottum detectors in TFTR ${ }^{91.94}$ More recently. MHD-induced losses have also been observed with the midplane detector. Illustrated in Fig. 6.2 is the signal from a low $q$ discharge (shot $\quad$ 70279, $R_{\text {aperture }}=350.5 \mathrm{~cm}$ ), along with the Mirnov coil trace which exhibits the presence of fishbone activity. The time correlation between the two curves is especially noteworthy and so is their relative amplitudas. In Fig. 6.3, we show a detail of the same two traces (but for a different shot ( $\$ 50278$ ), $R_{\text {aperture }}=352.5 \mathrm{~cm}$ ) where the modulation of the losses due to a $m=2, n=1$ mode is clearly visihle 

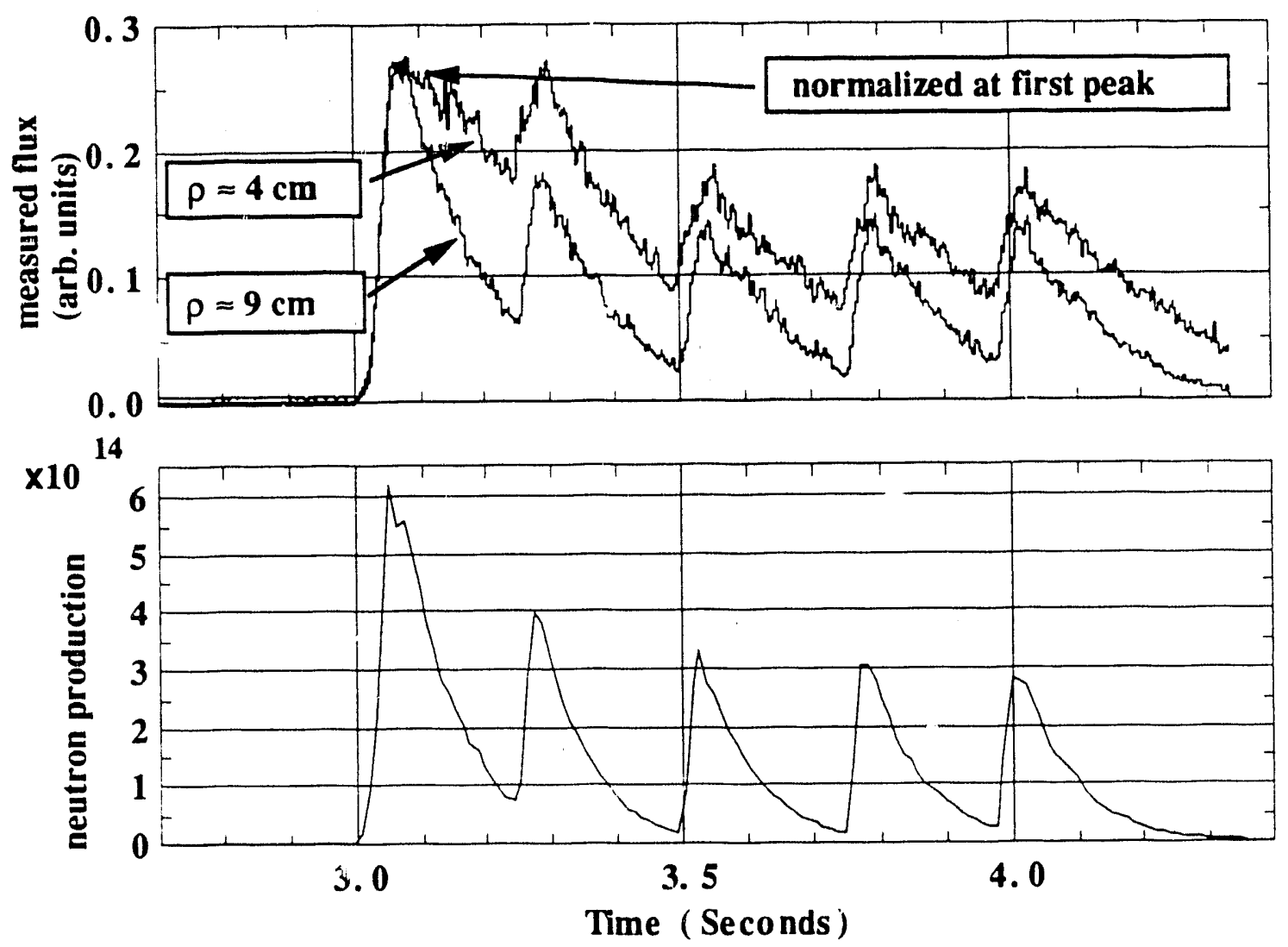

Figure 6.1: Measured flux of CFPs during short NBI blips at two different gyroradii (top tuo traces) and for neutron production (bottom trace). The time dependence of the lower tnergy component $(\rho \approx 4 \mathrm{~cm})$ dijfers significantly from the birth energy component $(\rho \approx 9 \mathrm{~cm})$ for the same pitch angle $\left(\chi \approx 55^{\circ}\right)$. A possible slou diffusive mechanism may be operative here $\left(I_{P}=0.8 \mathrm{MA}, B_{T}=4 T\right.$ and $\left.R_{0}=2.42 \mathrm{~m}\right)$. 


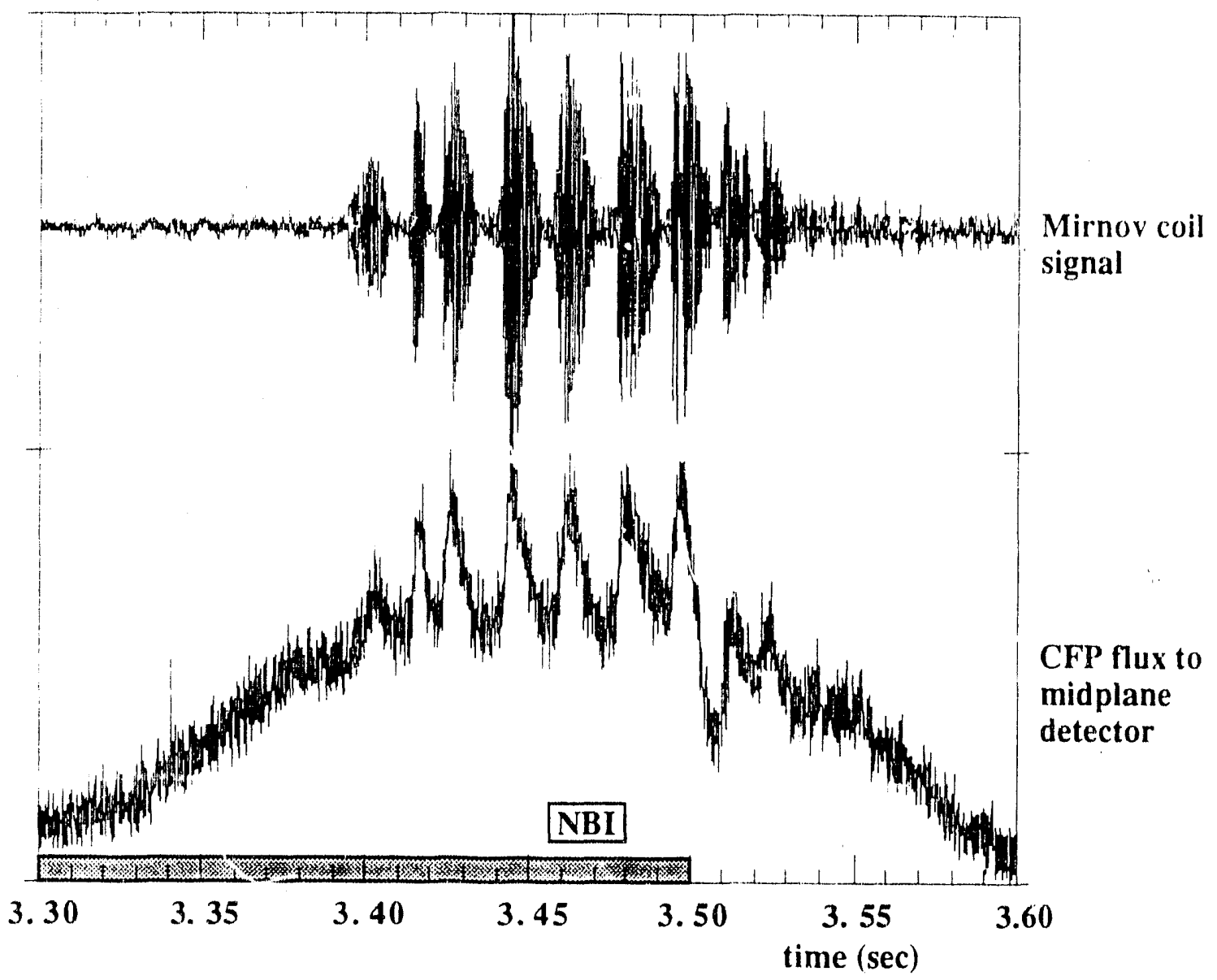

Figure 6.2: MHI)-miduced losses of (FP's during fishbone activity $\left(I_{p}=1.1\right) \mathrm{MA}$, $B_{T}=3.47$, stoot $\# 5(1379)$. On top, the Mirnor trace and below, the escaping flux recorded by a photomultiplier tube. 


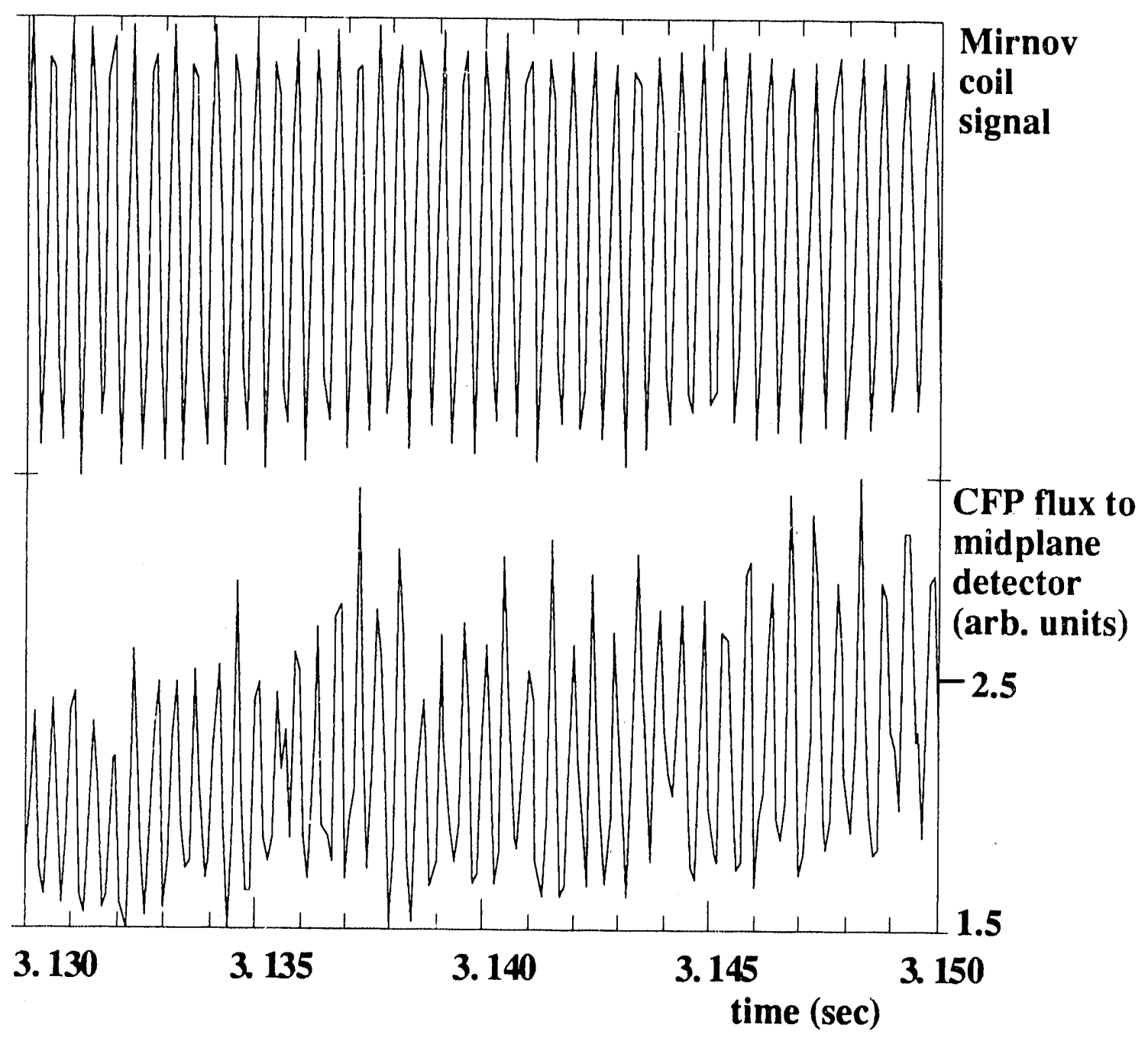

Figure 6.3: MHD-induced losses of CFPs during an $m=2, n=1$ mode $\left(l_{p}=1.0 \mathrm{MA}\right.$, $B_{T}=3.4 T$, shot \#50278). On top, the ilirnov trace and on bottom a blown-up time interval of the measured CFP flux showing the correlation with the MHD activity. 
We saw that loss processes like TF ripple and MHD-induced losses can be serious concerns for the confinement of CFPs in tokamaks. We must also be concerned by the possibility of the conjunction of different processes which could lead to more losses than the two processes taken separately. Strictly speaking, TF ripple losses are an example of synergistic effects between direct (first-orbit) losses and magnetic field perturbations. Consequently, MHD activity can open regions, composed of previously confined particles, to existing loss cones like first-orbit or even ripple losses. For example, the presence of MHD activity can cause a number of passing particles to become trapped, ${ }^{19}$ which would then be lost if the banana width is too large (equivalent to first-orbit losses) or, if their bounce point lies in the TF stochastic ripple domain.

In addition the MHD structure can create its own ripple (instead of $\mathrm{N}$ coils, the ripple would have a toroidal mode number ' $n$ ' and a poloidal number mode ' $m$ '), which can be sufficiently large to lead to stochastic diffusion. ${ }^{28}$ Although possibly only a local phenomenon around a resonance surface, the combination of this mechanism with TF ripple diffusion could lead to an unacceptable level of losses. A fluctuation level of a few percent is needed for leading to stochastic ripple diffusion. ${ }^{28}$ Some aspects of this mechanism will be briefly discussed in Ch. 7.

We believe we found, accidentally, a situation where MHD activity induced anomalous losses through two different channels in the same shot, the first-orbit channel (in fact, at the fattest banana) and the TF ripple channel. In Fig. 6.4 we show the flux measured at the midplane for the shot \#53457. First, we plotted the pitch angle distribution for two different times. In the earlier distribution the peak due to first-orbit losses is smaller than the second peak, which is attributed to TF ripple losses. In the later distribution we observed the opposite. The gain of the camera was kept the same throughout the shot. It is also interesting to follow the time dependence for those two pitch angles which are shown in Fig. 6.5. For the first-orbit loss peak we see that anomalous losses occur after $4.3 \mathrm{sec}$ but for the "ripple" peak they occur just before 4.3 msec. The mechanism is definitely not understood, the dynamics involved here is certainly complex. Unfortunately the data from the Mirnov coils happened to be of relatively poor quality on that shot (noisy) and details of the MHD activity could not obtained. However the possible presence of a locked mode unlocking at 4.3 sec has not been ruled out. 


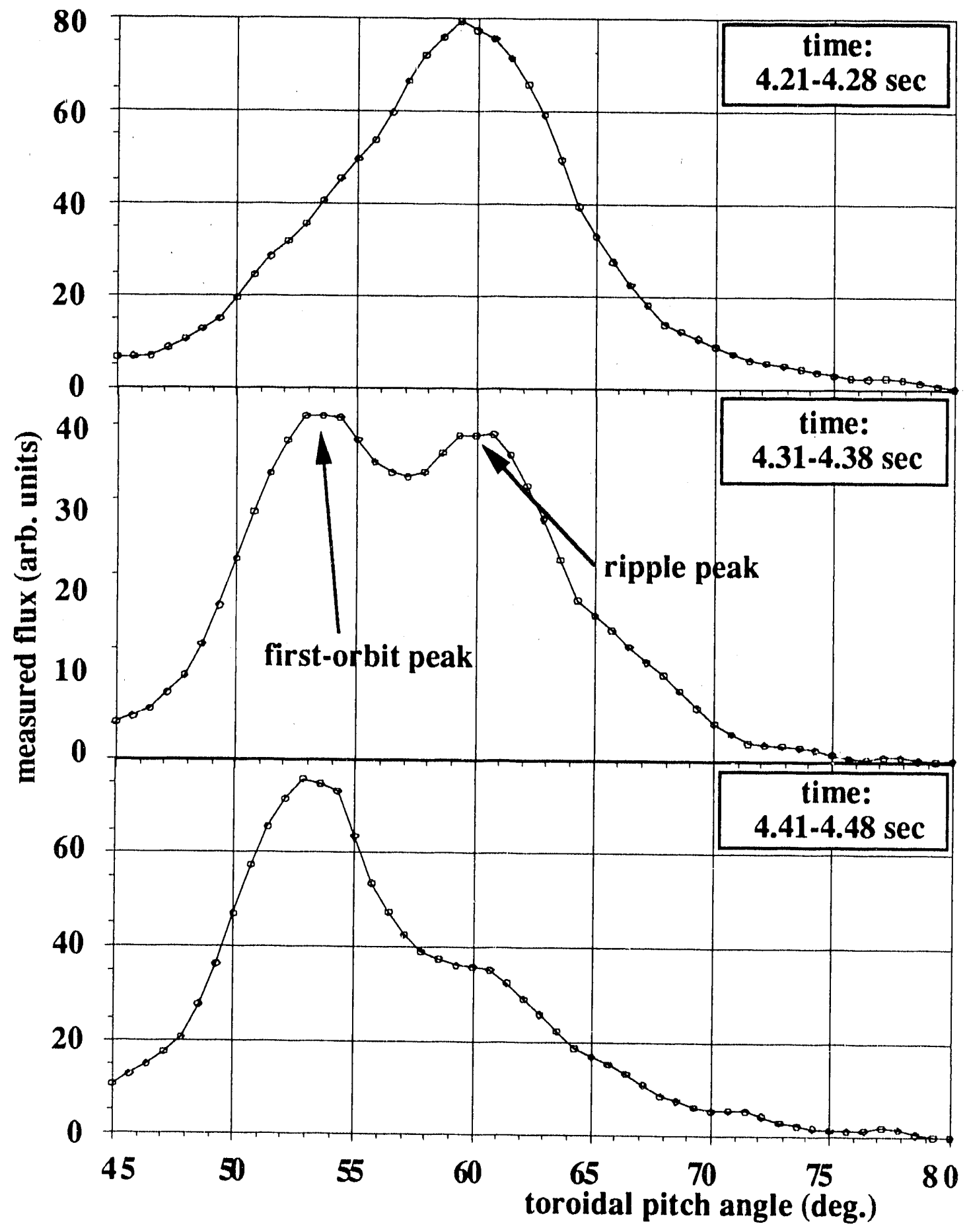

Figure 6.4: Pitch angle distribution during MHD activity (60 msec apart). The peak on the left is attributed to first-orbit losses (fattest banana) whereas the right peak is attributed to TF ripple losses. 


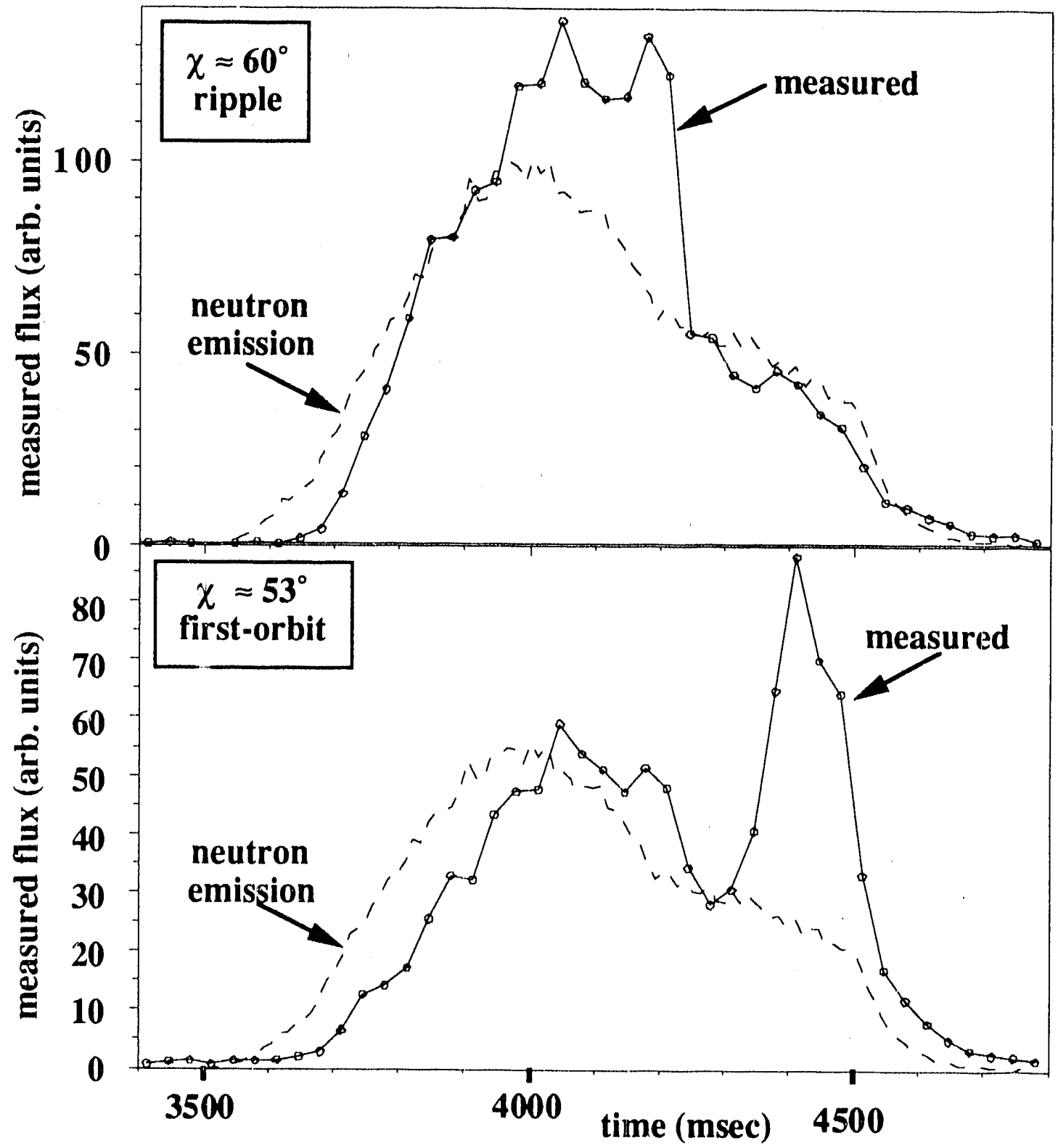

Figure 6.5: Time dependence for two different pitch angles (first-orbit on the left, ripple on the right) during $M H D$ activity, shouing the transition at $4.3 \mathrm{sec}$. Also shown in dash line, is the time dependence of the source (normalized at $3.4 \mathrm{sec}$ ) indicating the presence of anomalous losses. 


\subsection{Sawteeth-induced losses}

It is been observed previously at the bottom of TFTR ${ }^{93,94}$ and at the top of JET ${ }^{59}$ that bursts ( $\leqslant 1 \mathrm{msec}$ ) of escaping CFPs were sometimes recorded at the sawtooth crash. It is been conjectured that the CFPs have been expelled from the center of the plasma during the sawtooth crash. In TFTR, these observations were, until now, only made at one detector $\left(\# 6\right.$ at $\left.\theta=-90^{\circ}\right)$. However, as photomultiplier tubes are necessary to observe these fast bursts, little time was spent on the other detectors to look for these effects and so may they have been inadvertently missed. When the photomultiplier tubes system was used for the midplane detector, we observed occasionally some bursts of escaping CFPs during a sawtooth crash.

In Fig. 6.6 is shown a fast burst of CFPs similar to what as been observed previously. The burst is fast ( $\sim 1 \mathrm{msec}$ ) and has been observed to be maximum at $\chi \simeq 55^{\circ}$; the pitch angle corresponding to the fattest banana. In this case the flux increased by $30 \%$ over the previous level (shot $\# 50282, R_{\text {aperture }}=343.5 \mathrm{~cm}$ well inside the RF limiters).

The physical picture of the effects of sawteeth became more complex when a different behavior was also found during a sawtooth crash. In Fig. 6.7 is shown the total escaping flux to the midplane detector during a sawtoothing discharge. In this shot the camera was used, nevertheless the effects of the sawteeth are visible because of the very long time evolution of the losses ( $150 \mathrm{msec}$ comparatively to $\lesssim 1 \mathrm{msec}$ in the previous case). The central electron temperature is also displayed, showing the time correlation between the crash and the increase in escaping flux. This suggests that for some conditions, changes in the escaping flux would originate from modifications of the source and current profiles. The pitch angle distribution was not found to vary significantly during the crashes. Further study of the sawtooth mechanism by looking at the losses of CFPs to different detectors is being planned for the future. 


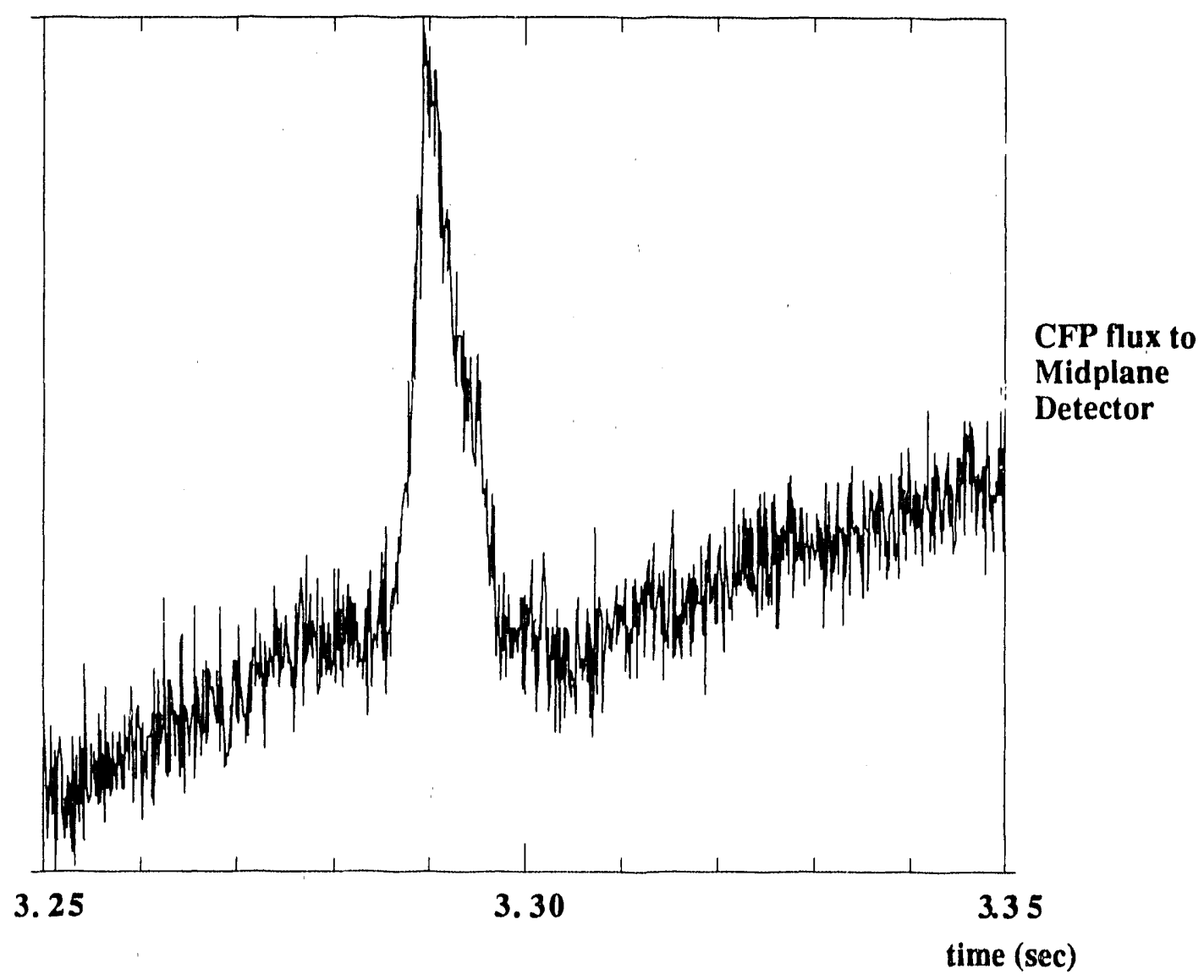

Figure 6.6: Burst of escaping CFPs (increase of $30 \%$ in signal) observed with the midplane detector with the photomultiplier tubes systern during a sawtooth crash. 


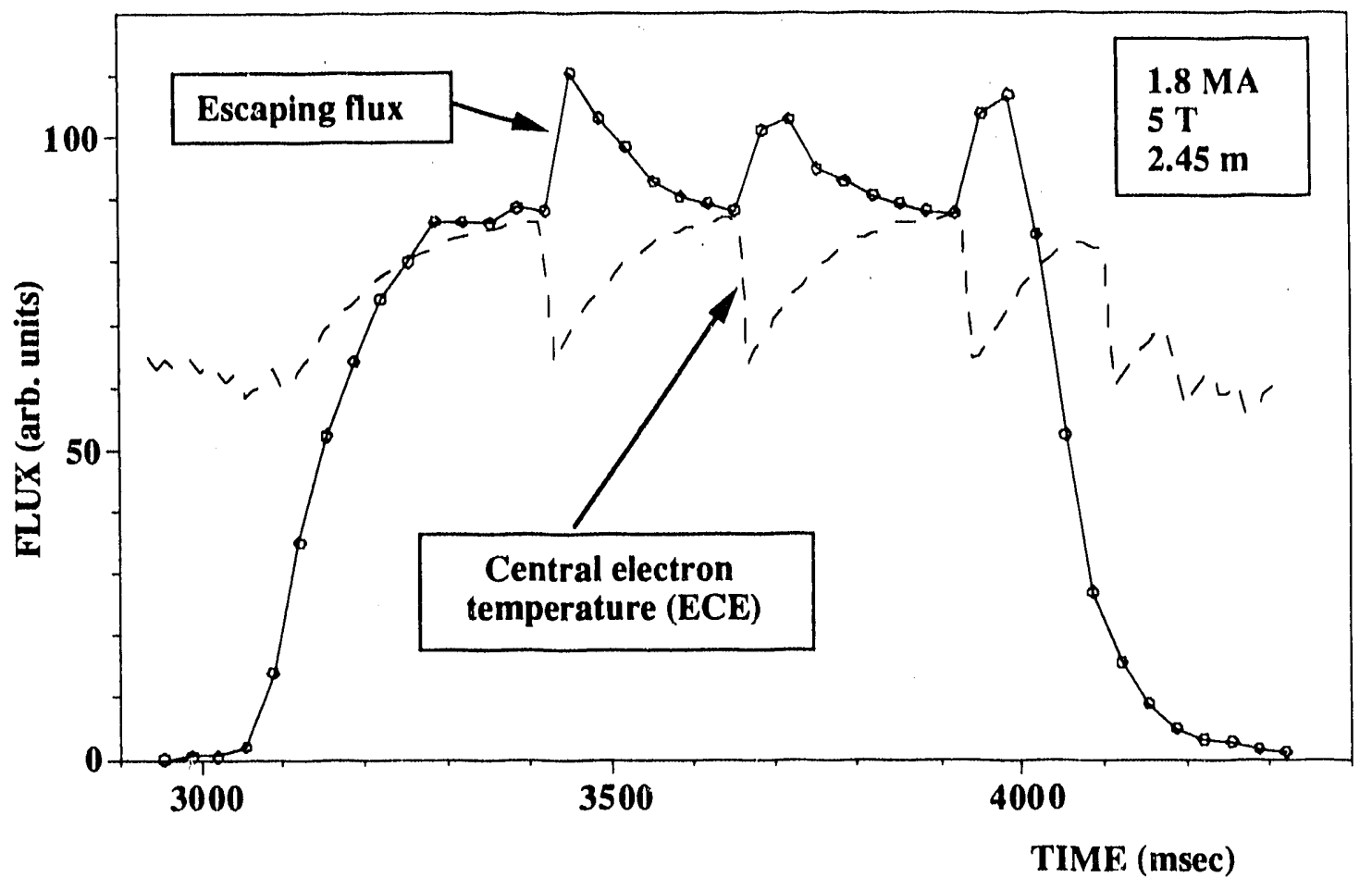

Figure 6.7: Modulation of CFP losses at the midplane detector during sawteeth. Also shown is the electron temperature measured by the ECE technique near the center of the plasma. $\left(I_{p}=1.8 \mathrm{MA}, B_{T}=5 \mathrm{~T}, R_{0}=2.45 \mathrm{~m}\right)$ 


\section{Chapter 7}

\section{Suggestions for future work}

As we mentioned previously, tokamak alpha particle physics should be a topic of increasing importance in the years to come, especially with the up-coming usage of tritium as a fuel. We explored one aspect of the single-particle confinement of CFPs, namely the phenomenon of diffusion due to Toroidal Field ripple. We expect that this phenomenon will have a significant effect on the design of fusion reactors. However, there are still other ripple loss mechanisms that need to be addressed as described in Sect. 2.1.2 and 2.1.3. We will propose some other possible avenues for research and describe their connections with the present results. Some possible improvements in cur analysis of the previous chapters will be described first. In Sect. 7.3 we will also take a brief look at the extension of this work to MHD-ripple induced losses.

\subsection{TF stochastic ripple diffusion}

It became rapidly apparent in C'hapter 4 that the predominant uncertainties were due to the first wall geometry, which also includes the detector and its probe support. This is certainly a relevant question for the future since localized heat loads due to anomalous losses (e.g. ripple losses of all types) of alpha particles would be magnified by any non-uniformity of the first wall.

One of the reasons why we were able to obtain detailed numerical calculations of CFP ripple losses is due to our use of a mapping technique rather than the full guiding-center approach. However, when a particle gets closer to a wall we had to revert to the guiding-center equations, which slows the computations quite exten- 
sively, especially if the first wall geometry is composed of many different elements. Since our measurements are made by a movable detector, we also had to establish a correspondence between calculated losses to the wall and to the detector.

One possibility for clarifying this issue would be to combine the numerical approaches of Chapters 4 and 5. For example, we developed in Sect. 5.2.2 a technique to calculate the flux of particles to the detector as a function of its position behind the RF limiters. We saw that the fall-of rate could be modeled by TF ripple diffusion. However, the calculations were done for one pitch angle only and were normalized at one specific detector location, somewhat arbitrarily. The code MAPLOS could be used here to normalize the radial fall-off curves at the wall, where losses are known as a function of pitch angle. Consequently one could simulate the pitch angle distribution and calculate the total flux to the detector by establishing the fall-off curves for different pitch angles. One could then obtain the evolution of the pitch angle distribution as a function of the detector position and compare, for example with Fig. 4.16. The additional flux of particles to the detector due to that radial dependence would probably fill the gaps shown in Fig. 4.10 and 4.17.

At the second level of uncertainties, many factors contribute to the accuracy of the calculations. The principal ones though are tied to our knowledge of the current and source profiles, where the former is still a poorly known quantity. Consequently, at the present moment, improvements in the physical representation of the plasma and its parameters (e.g. Shafranov shifts, particle slowing down, etc.) are not- yetworth the efforts.

\subsection{Ripple trapping}

One question that remains open with respect to the confinement of CFPs in the presence of $T F$ ripples is naturally the amplitude of ripple trapping losses. We saw in Sect. 2.1.2 that ripple-trapped particles can exit the plasma very rapidly because of the uncompensated drift due to the presence of the ripples. We also saw that the region of trapping is usually small, or does not contain many particle bounce points (for example compare Fig. 2.5 and Fig. 4.3 which do not overlap significantly). However, for the case of ITER, ${ }^{6}$ even a small region at the midplane near the outer edge of the plasma could mean additional flux of particlos to the wall. This represents another serious heat load problem since the lost alphas would not only be poloidally local- 
ized (above/below the midplane) but also toroidally localized, between two toroidal field coils at a minimum $B$. The problem can also be enhanced by the nearby presence of the TF stochastic ripple region with which particles could be exchanged. thus increasing the loss rate.

As for the case of TF stochastic ripple diffusion, the study of ripple-trapped losses needs specialized detectors. Since the trapping region is limited mainly to the outer edge, only particles born with a pitch angle near $90^{\circ}$ (virtually no parallel velocity) could be ripple-trapped.

That means that particles mainly drift vertically (a fraction of centimeter per gyromotion) and consequently the aperture must be located very near the top of its casing. If not, particles would be scraped off (by the detector armor for example) before they could be detected. This is the fundamental reason why the detector that we used so far, described in Sect. 3.1.1, could not look at this class of particles. In fact the maximum "visible" pitch angle was established to be approximately $80^{\circ}$.

In Fig. 7.1 we show the out line of a detector specialized for the study of rippletrapped losses of CFPs which is somewhat similar to a prototype of the lost alpha detectors installed on TFTR in $1986 .{ }^{139}$ As we mentioned above the main difficulty. in the design resides in the positioning of the aperture sufficiently close to the top to the casing. The situation is sornewhat facilitated for particles not exactly at $90^{\circ}$ in pitch angle which can approach the detector from the side. For example. a 3 Mel proton at $85^{\circ}$ in pitch angle can still travel approximately 2 to $3 \mathrm{~cm}$ parallel to the field line over a full gyroperiod.

The apertures can be made relatively big in order to maximize the flux of particles. The first aperture would be positioned far from the scintillator in order to block any direct line of slight (preventing X-rays backgrounds). Light from the scintillator could be relayed from the back of the scintillator by a mirror or a guide light to fiber optics at the bottom of the detector. A relatively thick aluminum foil $(20 \mu \mathrm{m})$ would be used to block stray light and particles and would limit the access to the $3 \mathrm{MeV}$ proton. Shielding would be minimum since the detector can be installed much lower (between two field coils) than the last closed flux surface. If the expected particle flux is not large enough for using the scintillation technique, the scintillator could be replaced by a simple silicon sample and left exposed for long periods of time (weeks or months). ${ }^{\text {i0 }}$ 

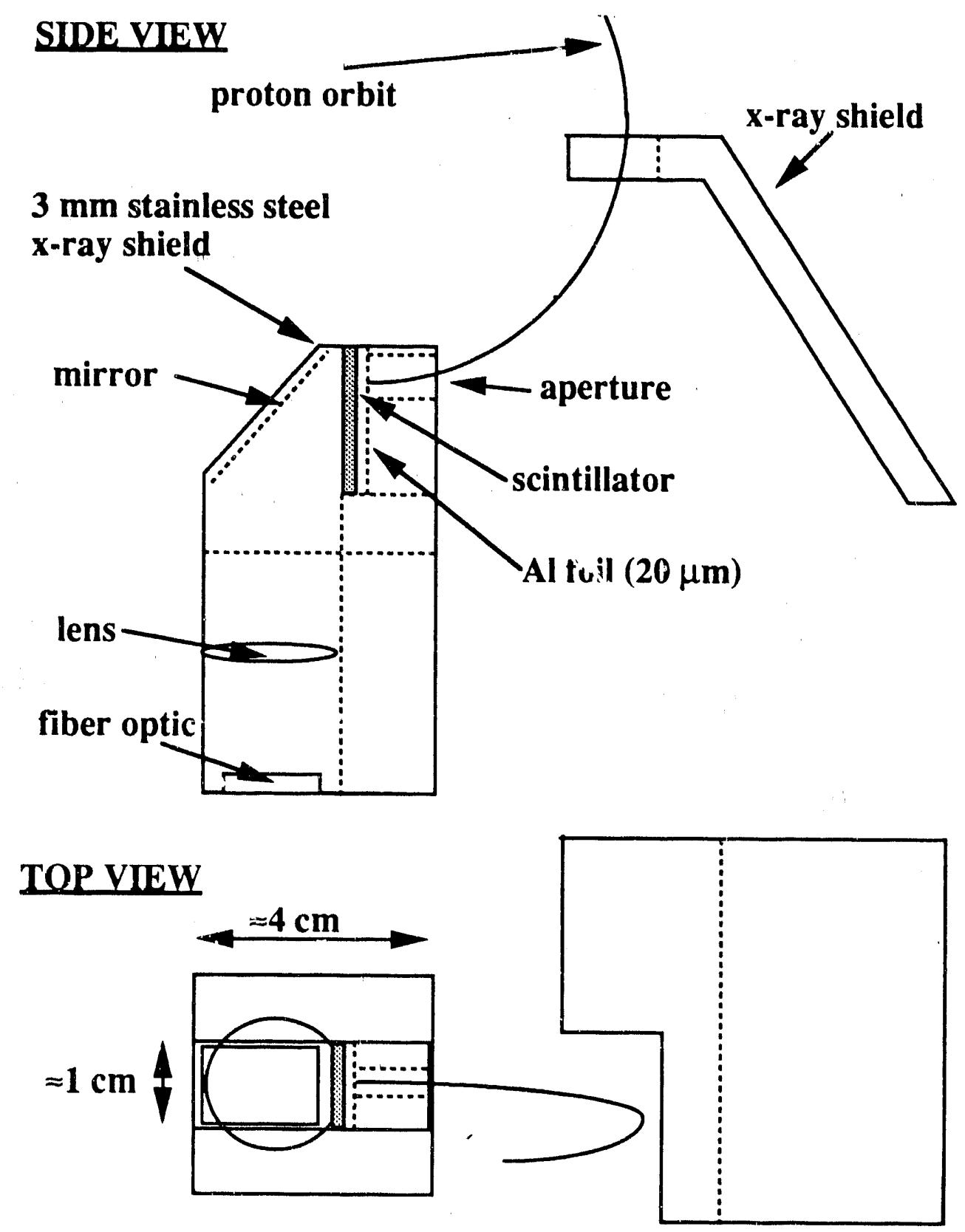

Figure 7.1: Conceptual design of a detector for the study of lost ripple-trapped CFPs. Ripple-trapped particles drift vertically in between toroidal field coils at a pitch angle near $90^{\circ}$. 


\subsection{MHD ripple-induced losses}

The effects of MHD activity on the single particle confinement of CFPs is receiving an increase attention from researchers. This interaction which could take several forms, has still an unknown effect on the global confinement of CFPs and on possible additional first wall heat loads. A certain theoretical understanding ${ }^{19,} 30,140$ is emerging from the interaction of coherent modes, i.e. internal kinks, fishbone-type activity or TAE mode, with passing particles. This process simply brings passing particles, near the passing-trapped boundary, slightly away from their birth flux sur face. Some of these particles would make large enough excursions that they would become trapped and quickly lost through a first-orbit channel.

However, there is a second possible interaction, this time directly with trapped particles. We know that MHD activity can create its own magnetic ripples (contrary to $\mathrm{TF}$ ripples they would be internally created) and consequently all the mechanisms described in Sect 2.1.2 and 2.1.3 could contribute as well to diffusion and CFP losses. Of particular interest is the collisionless stochastic diffusion (MHD equivalent to the TF stochastic ripple diffusion) where the number of coils (for TFTR $N=20$ ) can be replaced by the toroidal mode number of the mode. ${ }^{28,29}$ However, by using Eq. 2.8 we see that even with large amplitude modes (or large $\delta$ ) the process would be inoperative because of the low n number.

This phenomenon could be related to the multiple occurrences of semondary peaks at the bottom detectors. Those secondary peaks ${ }^{34}$ appeared at lower energy $(\sim 1.5$ $\mathrm{MeV}$ for the proton) and at a pitch angle corresponding to deeply trapped particles. These occurrerices seemed to be related with the presence of MHD activity and were not observed at the midplane detector.

Finally, there exists a third connection which did not receive full attention yet. We saw that ripple trapped losses should normally be small, at least in TFTR; the small amplitude of the ripple being the main reason. However we know also that MHD activity can create fluctuations close to a few percent right in the middle of the plasma. Since the particles bounce frequency (typically $100 \mathrm{kHz}$ ) is usually larger than the mode frequency (especially in presence of locked modes), partic: as could be trapped in the mode ripple and could drift out significantly, although without necessarily being lost. 


\subsection{ICRF high energy minority tail ions}

Alpha particle physics can also be studied through the high energy tail ions created by ICRF heating. In TFTR, when the hydrogen minority heating scheme was used (especially at lower density), high energy protons $(\sim 1 \mathrm{MeV})$ were detected in large number towards the outer midplane. Since the heating occurs at the bounce point of trapped particles, they would be especially susceptible to TF ripple diffusion. The flux has been found to be quite large at the midplane detector and normall, was decreasing towards the inner midplane although when the resonance layer was moved further inside the maximum of flur was found to be at the $\theta=-60^{\circ}$ detector.

One other interesting feature was found at the $\theta=-45^{\circ}$ detector at larger $\mathrm{RF}$ power $(>2 \mathrm{MW})$ in the presence of sawteeth. The signal was found to be modulated by the sawteeth. In Fig. 7.2 we show such a case where the flux of high energy protons drops to almost zero with the sawtooth crash. In this case the sawtooth crash may change the $q$ profile to the point of shutting down ripple losses (the $q$ profile, by becoming flat, would change the stochastic threshold significantly). It could be explained also by an expulsion of fast ions at the crash, consequently removing the tail from the resonance layer. The repopulation of the tail would take some time, at which point the discharge is ready to crash again. 


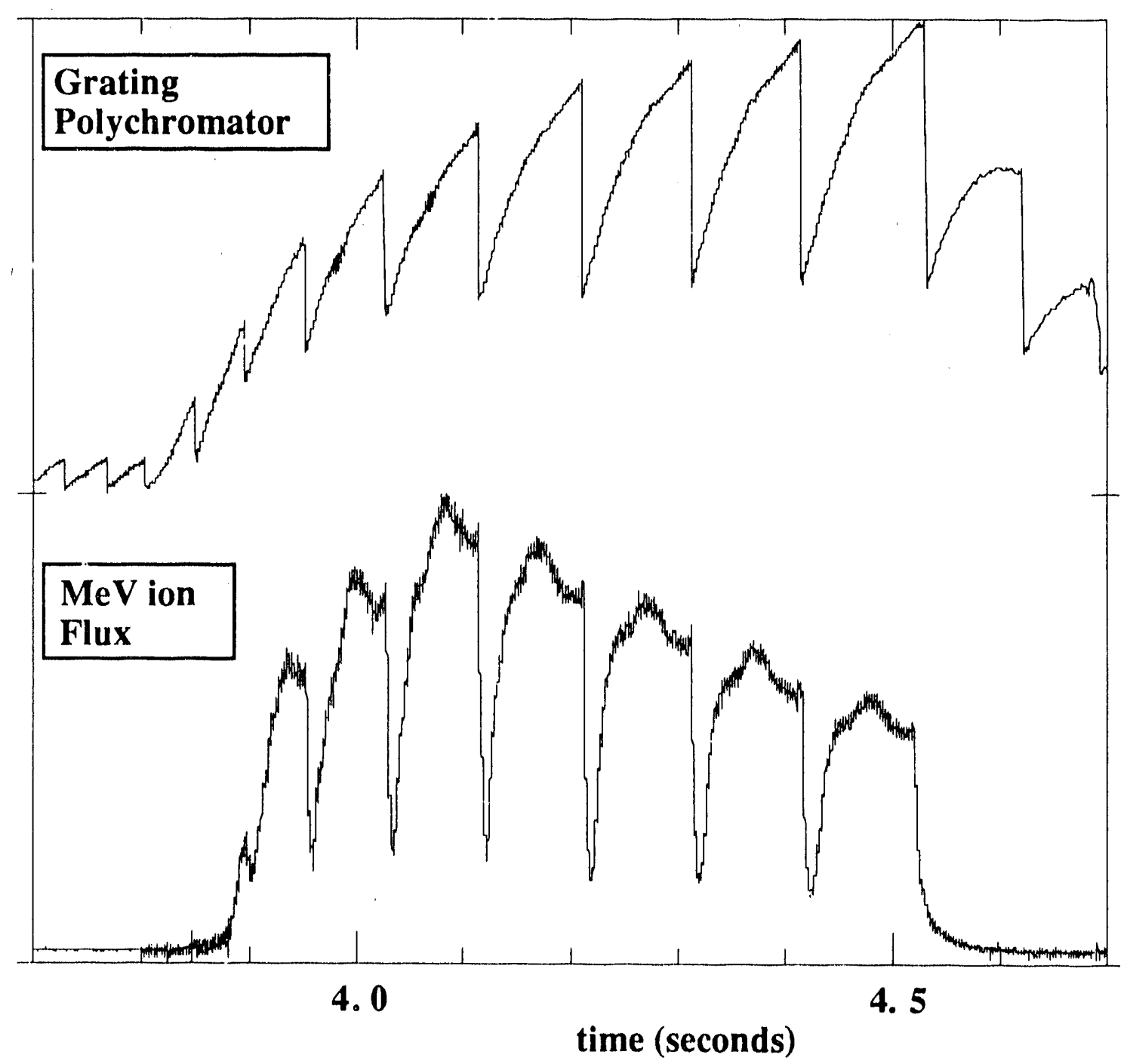

Figure 7.2: RF minority tail ions signal at the $\theta=-45^{\circ}$ detector during a sawtoothing discharge. Also shown are the electron temperature fluctuations near the center taken by the grating polychromator. 


\section{Chapter 8}

\section{Conclusions}

Vie presented the first experimental evidence for the toroidal field stochastic ripple diffusion of charged fusion products (CFPs) and compared the results with the theoretical model originally proposed by Goldston, White and Boozer. ${ }^{2}$ From theoretical considerations we established the following signatures of TF stochastic ripple diffusion of CFPs:

i. TF ripple diffusion affects trapped particles only.

ii. Ripple diffusion exhibits a spatially localized stochastic threshold. Diffusing particles are generally deeply trapped (but not ripple-trapped).

iii. Diffusion rate is relatively large, loss time is small compared to thermalization time.

iv. The losses are locali'ved poloidally (just below outer midplane).

v. The losses have a strong current dependence.

vi. The diffusion rate is set by external parameters (ripple amplitude, plasma current, plasma major radius, etc.).

Since these losses were expected to be highly localized, we installed on TFTR a radially movable detector just below the outer midplane. The detector design is based on a scintillation technique and has energy, pitch angle (magnetic moment) and time resolution. Various plasma conditions were studied which permitted us to observe the presence of that theoretically predicted loss mechanism. We also developed a 
mapping code (MAPLOS) for calculating loss distributions of particles (including the particle pitch angle distribution) at the first wall of TFTR.

Evidence for point [i.] was described in Chapter 5 where we found that the detected particles were diffusing trapped CFPs. We excluded the possibility that the anomalous flux was due to diffusing passing particles since the pitch angle of the escaping CFPs was clearly away from the passing/trapped boundary (see Fig. 4.11). Also, passing orbits are located well inside the plasma, and could not have been blocked by the obstacle or the detector during our shadowing experiments.

The presence of a well delimited stochastic threshold (point [ii.]) was shown in Fig. 4.14 and also in Fig. 4.11 and in Fig. 4.13. The clear resolution (Sect. 4.3.2, through the pitch angle distribution, of two classes of lost particles was explained by the spatially separated regions of first-orbit and ripple losses. Numerical calculations based on the TF ripple diffusion model were able to simulate quantitatively the experimental results. The predictions of the peak in the pitch angle distributions also matched the experimental results over a wide range of plasma conditions. The inclusion of the RF limiters in the calculations improved the agreement, especially at higher current where the diffusion rate is smaller.

We saw also that the presence of a spatially localized threshold would prevent many particles from diffusing, which otherwise would escape at pitch angles ranging in between the two observed peaks (Fig. 4.14). Those pitch angles correspond to orbits originating from a section of the plasma of minimum ripple amplitude, region theoretically expected to be free of stociastic ripple diffusion. Experimental results were found to be consistent with the model proposed by Goldston, White and Boozer but also with the more optimistic model oi Grua and Roubin which differs by a factor of 1.8 for the stochastic threshold.

The points [iii.], [iv.] and [vi.] are certainly connected. By using all four detectors, we observed that an additional flux was reaching the wall near the midplane (point. (iv. f), in a semi-quantitative agreement with theoretical simulations. We found also that not only was the diffusion rate consistent with theoretical expectations (Sections 5.2.3, Fig 5.10 and 5.2.2, Fig. 5.8), but that it was also very reproducible over different shots and over different run days, even with months apart (Fig. 5.4). The agreement with the theoretical expectations was found to hold even in the relatively different shadowing experiments (small and large obstacles). 
We found in Sect. 4.3.1 that the current dependence of the measured losses was at least qualitatively consistent with the presence of ripple diffusing particles, especially because of the expected peak at medium current. This behavior was found for both aspect ratios (Fig. 4.10), in which the experimental results exhibited a local maximum expected from numerical calculations. The measured flux was clearly away from calculations based on a first-orbit loss process only. In loss mechanisms other than TF ripple diffusion (and first-orbit), it is not expected that the losses would be strongly dependent with current (point [v.]). It is clear that any type of anomalous losses would be small at low current since most of the particles are lost on their first orbit. However, at high current we observed that losses were decreasing after $1.4 \mathrm{MA}$ especially in the $R_{o}=2.45 \mathrm{~m}$ case in which losses decrease significantly with current. If a loss mechanism (different than ripple diffusion) was still operational at the diffusion rate found in Chapter 5 losses (e.g. at 1.8 MA and higher) would be much larger than observed.

The reproducibility of the results and the consistency of the agreement with theoretical expectations exclude for the majority of the studied cases the possibility of coherent MHD-induced diffusion. This is also supported by the fact that when studying cases which were not low $q$ discharges and when fast time response was used (with the photomultiplier tubes) we found no indication of modulated losses (up to $100 \mathrm{kHz}$ ). We also did not observe any sign of significant losses of slowed down particles during steady-state (Fig. 4.18), especially at the pitch angle where anomalous losses were observed.

One immediate consequence of this experiment concerns the global TF stochastic ripple losses of CFPs in TFTR. We found that the experimental results were consistent with the Goldston, White and Boozer model which predicts a maximum of $5 \%$ loss fraction. This level would not be sufficiently large to explain the observed low triton burn-up. ${ }^{69}$ This also means that for the D-T phase of TFTR, TF ripple losses should not prevent the study of collective effects from alpha particles (and even less for the study of the toroidal Alfvén eigenmode [TAE mode], which interacts predominantly with passing particles).

However this does not exclude the possibility of synergistic effects, for example, between TF stochastic ripple diffusion, ripple trapping, sawteeth and MHD activity. This category of effects would be part of the next logical steps in the study of single particle confinement of CFPs, especially in TFTR. 
Finally, this experiment has long term consequences, namely in the design of fusion reactors such as BPX and ITER. We showed that there is a need to take into account the effects of the toroidal field stochastic ripple diffusion in their design. Different threshold coefficients (within a factor of 2 of the Goldston, White and Boozer criterion) should be considered in their numerical analysis. In addition, the delicate problems of first wall heat loads will necessitate a detailed analysis of the interaction of the first wall geometry with ripple losses of CFPs, such as developed here for the in the interpretation of the experimental results (MAPLOS). 
'It is a far, far better thing that I do, than I have ever done;

it is a far, far better rest that I go to than I have ever known.'

Charles Dickens, A Tale of Two Cities, Bk.iii,ch. 15. 


\section{Appendix A}

\section{Code descriptions}

In this chapter we describe briefly the different steps and approximations found in the codes (written in FORTRAN) that we used for numerical simulations. This will also permit a more global view of the uncertainties involved in those calculations. In the first section, we will describe MAPLOS which was used to calculate the different loss distributions from first-orbit and TF stochastic ripple losses. In the second, we will describe the two simpler codes used in Chapter 5 for the simulation of the shadowing experiments. Finally; will review briefly the Lorentz code ORBIT.

\section{A.1 MAPLOS}

The basic idea behind a code like MAPLOS, consists mainly in following the orbits of CFPs, sufficiently long enough (ideally until they thermalize) until they are determined to be confined or lost. It, is obvious that following $10^{20}$ particles (e.g. $3.5 \mathrm{MeV}$ alphas) for approximately 1 second using a detailed description of the toroidal and poloidal fields is an intractable problem even with today's supercomputers. Many simplifications are needed and we will attempt to describe most of them.

In our case, the first step in reducing the complexity was to reduce the number of particles to approximately 200000 . This was done by scanning through a grid of birth locations typically composed of 60 radial, 60 poloidal and 60 pitch angle points. The radial and poloidal distributions are uniform in the polar coordinates grid. The toroidal location was determined randomly. The birth pitch angle distribution is isotropic and is also jiggled slightly (small perturbations added to the isotropic 
distribution) in order to prevent aliases in the resulting distributions. This relatively large number of particles was needed in order to get good statistics for the various distributions especially for the pitch angle distribution which is calculated at a specific wall location.

Because of that large number of particles. we want to insure that the code will be fast enough. First of all, we integrate the guiding-center orbit only, treating gyroradius effects only when close to obstacles (i.e. walls). The calculations use the guiding center integration routine, developed by White and Chance. ${ }^{118}$ ' 1 he code solves the guiding center drift equations:

$$
\begin{aligned}
& \frac{d \theta}{d t}=\frac{\partial H}{\partial P_{\theta}}, \quad \frac{d P_{\theta}}{d t}=-\frac{\partial H}{\partial \theta} \\
& \frac{d \phi}{d t}=\frac{\partial H}{\partial P_{\phi}}, \quad \frac{d P_{\phi}}{d t}=-\frac{\partial H}{\partial \phi}
\end{aligned}
$$

where we implicitly assume that the parallel direction was along the toroidal angle $\phi$ only, where $P_{\phi}$ and $P_{\theta}$ are the cannonical momenta (toroidal and poloidal respectively) and where $H$ is the particle Hamiltonian given by:

$$
H=\frac{1}{2} \rho_{\|}^{2} B^{2}+\mu B,
$$

where $\rho_{\|}$is the parallel gyroradius, $\rho_{\|} \equiv v_{\|} / B$.

The differential equations involving the different canonical variables and momenta, $\theta, \phi, P_{\theta}$, and $P_{\phi}$ can then be derived from Eq. A.1 ${ }^{118}$. The time integration is performed by using a fifth-order Runge-Kutta process. ${ }^{118}$ Substantial simplifications come also from the simple, circular, concentric (low $\beta$ ) flux surfaces used for TFTR cases. For the problem at hand, we consider that particles are at fixed energy (monoenergetic and no slowing-down), and fixed magnetic moment (no collisions). The action of the TF ripples will enter only through the diffusion operator (see Eq. 2.5). The current profile is modeled by a simple parabolic dependence:

$$
J(r)=J_{o}\left(1-\frac{r^{2}}{a^{2}}\right)^{\alpha}
$$

where $\alpha$ is a parameter obtained from SNAP and/or TRANSP calculations. The source profile is vitually obtained from the Neutron Multi-channel Collimator and can be modeled by a parabolic: 
or Gaussian fit:

$$
S(r)=S_{o}\left(1-\frac{r^{2}}{a^{2}}\right)^{r}
$$

$$
S(r)=S_{0} \exp \left(-\left(\frac{r}{\sigma}\right)^{2}\right)
$$

where $\gamma$ and $\sigma$ are obtained from the experimentally found source profile width.

The TF ripple magnitude (see Fig. 2.8) is taken from a fit ${ }^{28}$ to the calculated vacuum ripple $e^{22}$ and is modeled by:

$$
\delta=\delta_{o} \exp \left(\frac{\left(\left(R_{o}+r \cos \theta-R_{\text {rip }}\right)^{2}+b_{\text {rip }}(r \sin \theta)^{2}\right)^{\frac{1}{2}}}{w_{\text {rip }}}\right)
$$

where $R_{o}$ is the plasma major radius, $R_{\text {rip }}=2.25 \mathrm{~m}$ is the major radius of the center of the ripples, $b_{\text {rip }}=1.1$ is the ellipticity, and $w_{\text {rip }}=0.185 \mathrm{~m}$ is the scale length of the ripples. Fig. A.1 show a comparison between the calculated vacuum ripple at the midplane and the above fit.

The block diagram shown in Fig. A.2 summarizes the algorithm that we used in MAPLOS for the calculations of ripple losses. By using the guiding-center equations, the first poloidal transit (also called the first orbit) is derived. At the moment of impact, first-orbit lost particles are eliminated and their impact location is recorded. Passing particles are also simply discarded. The remaining particles, trapped, firstorbit confined, are separated according to the threshold criterion 2.8. From that point, only TF ripple diffusing particles are kept and their bounce point is then followed. Because the expression for the step size (see 2.5) is only valid at $\theta_{b} \neq \pi$ and $\theta_{b} \neq 0$, first-orbit confined particles, born in the region near the midplane (in fact, a very small number), are also discarded.

Diffusion of the bounce point is then applied to the particles by using the expression for the step size (see Eq. 2.5). Since we are in the stochastic ripple regime, the toroidal angle becomes completely random (see also Fig. 2.7) except very near the threshold. Particles are simply not allowed to go in the non-stochastic region by simply "bouncing" them back towards the edge.

Up to this point, only the bounce point of the diffusing trapped particle was followed. For any positive step size (any increase in minor radius), the particle orbit is then followed from the bounce point up to the midplane, and if an impact occurs, its location is recorded. If not, the diffusion resumes until an impact is found. The 


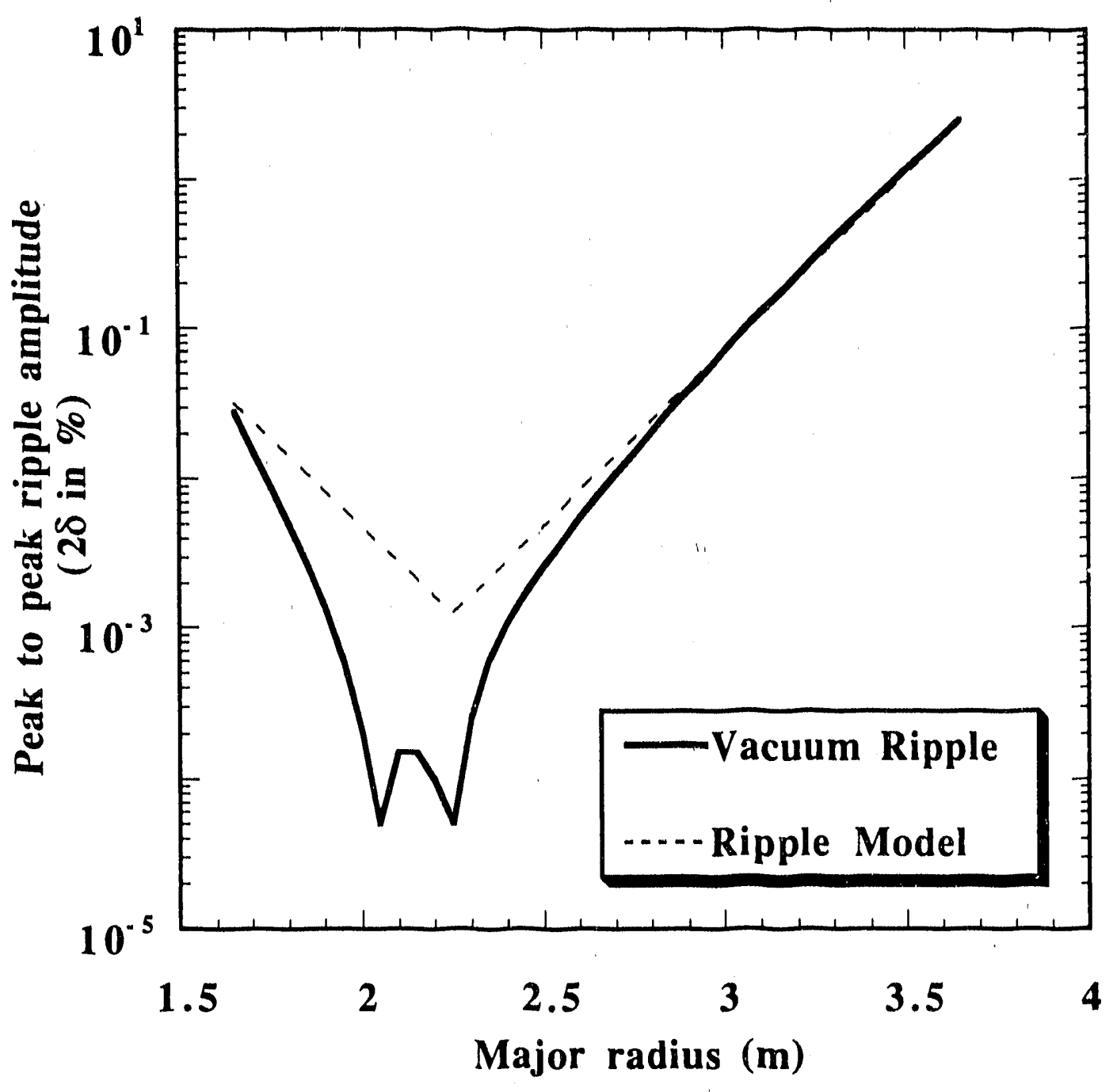

Figure A.1: Vacuum TF ripple amplitude (peak to peak $\equiv 2 \delta$ ) versus minor radius at the midplane compared to the numerical fit of Eq. A.6. 


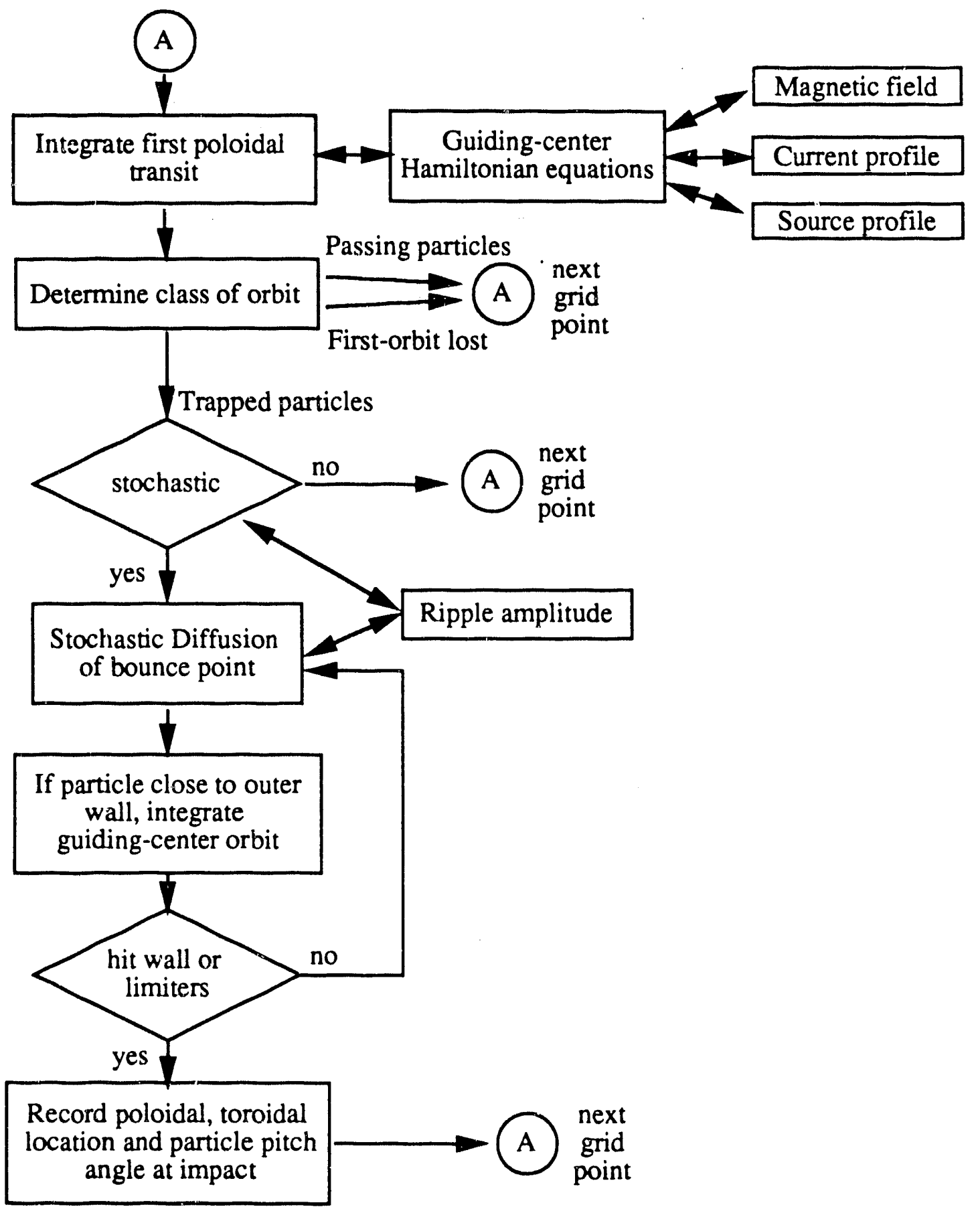

Figure A.2: Block diagram of the code MAPLOS illustrating the algorithm used to calculate ripple losses. 
wall is usually modeled as a simple circular surface and axisymmetric. However, cases have been run with the presence of the RF limiters in order to explore their effects of the different impact distributions on the wall. Future plans call for the addition of the detector as an obstacle and for calculations of the pitch angle distribution at the detector location, not just on the first wall. We show in Fig. A.3 a poloidal crosssection $^{141}$ of TFTR indicating the position of the different probes and also the major components of the first wall. Note however, that many of the features have a finite toroidal extension which is evidently not visible in this pcloidal projection.

This code was run on the CRAY - 2 and took typically between 80 and 130 minutes for 216000 grid points. Some exceptions were found at small major radii or when the threshold coefficient was increased artificially (increasing the number of lost particles).

\section{A.2 Diffusion codes}

\section{A.2.1 Diffusion in presence of a small obstacle}

As we mentioned previously, since both the detector and the obstacle are small in dimensions (e.g. poloidally), we can ignore the poloidal dimension and represent the object by a 2-D surface $(r$ and $\phi)$ in a plane located at the probe elevation. CFP orbits can then be simplified significantly and are modeled by a simple point in that plane corresponding to a crossing (for every complete poloidal transit or 2 bounce times). Consequently, we keep track of the guiding center of the CFP only and look at location of the plane crossing point.

The block diagram shown in Fig. A.4 illustrates the approach that we took for these calculations. First of all, the particle (of fixed gyroradius and pitch angle) is started at the detector aperture. The particle guiding center is recovered by simply removing one gyroradius from the radial position. For each passage at the detector elevation, the toroidal and the toroidal angles are chosen randomly and consequently determine the full radial position (with the gyroradius and the diffusing term). The particle position is then checked for any impact. If it hits the obstacle a flag is raised (but the particle not stopped) which signifies that all subsequent poloidal transits correspond to particles blocked by the obstacle only (see Fig. 5.5). Typically runs use approximately 10000 particles. 


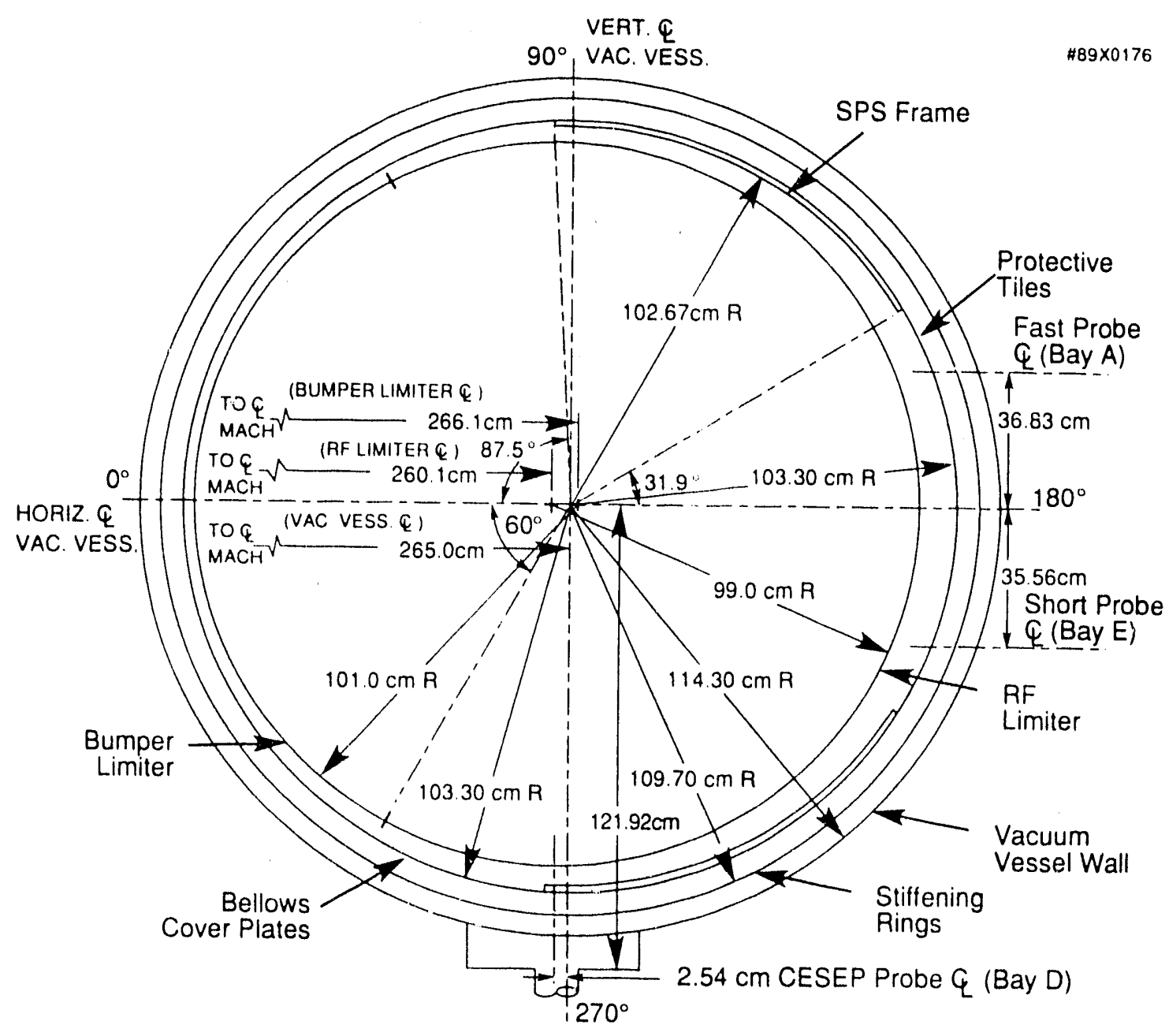

Figure A.3: Poloidal projection of TFTR cross-section indicating the location of the various probes and limiters. ${ }^{141}$ 


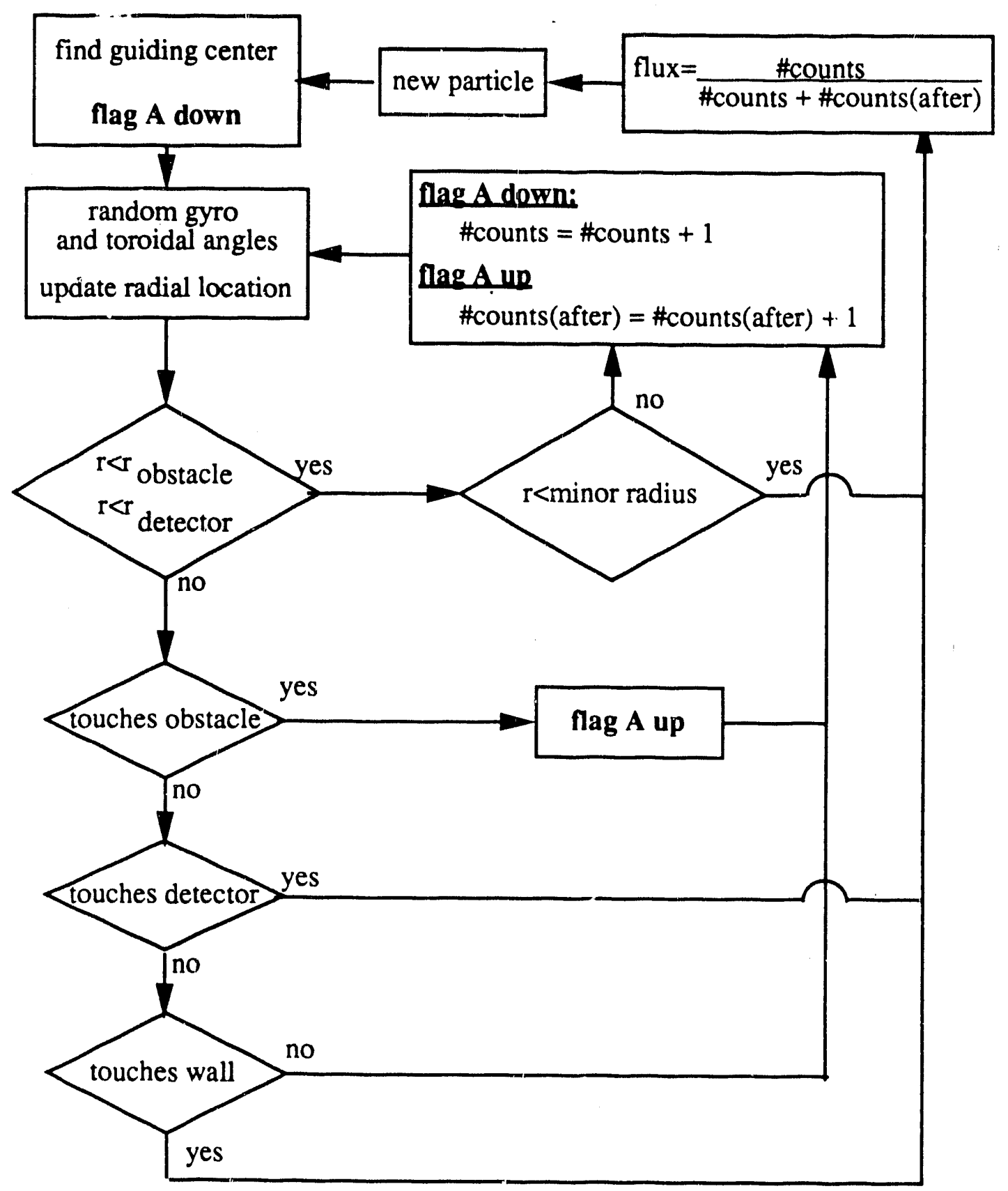

Figure A.4: Block diagram for the small obstacle diffusion code. A flag is raised when the particle hits the obstacle. The flux blocked by the obstacle corresponds to the number of poloidal transits made with the flag up. 


\section{A.2.2 Diffusion in presence of a large obstacle}

The approach used for the case of a large obstacle resembles the code described above. Since the obstacle, the RF limiters, have a large toroidal and poloidal extensions, an impact can occur at many locations and the particle orbit must be followed more closely. Using the Lorentz ORBIT code, we fitted the orbit by a quadratic expression giving the radial position as a function of $\theta$. The analytic expression for the orbit enabled us to simplify the calculations for the particle position when looking for a possible impact. Naturally, this fit was good only for a given set of plasma conditions.

We show in Fig. A.5 the block diagram for that code which again uses approximately 10000 particles. The major difference from the previous diagram consists in the addition of an inner loop where the banana orbit is followed for a possible impact. The orbit is followed from the bottom bourice point up to the top one. The toroidal angle is simply ralculated using the local $q=d \phi / d \theta$.

\section{A.3 ORBIT code}

The orbit code used to calculate first-orbit losses and absolute efficiencies of the various detectors has been described in the literature. ${ }^{53,101}$ However, it would be appropriate to review briefly the calculations of the detector efficiency and to verify its accuracy for our detectors. ORBIT integrates the particle orbit (keeping $E$ and $\mu$ constant) backwards in time from the slit inside the detector, through the front pinhole. It uses the full Lorentz force equation based on a divergence-free magnetic field which includes the poloidal field.

As we saw in Sect. A.l the particle equation of motion can be derived from an Hamiltonian which implies that phase space is conserved along the particle trajectory. That conservation of phase space is equivalent to say that the six-dimensional volume "seen" by the detector (i.e. the slit and pinhole) is equal to the six-dimensional volume of the plasma that produces the C.FPs reaching the detector. ${ }^{52}$ This equivalence of volumes is used for calculating the total efficienry of the detector,

$$
\epsilon=\frac{A \int d \Omega T(\Omega) \int S d l}{4 \pi \int S d V}
$$




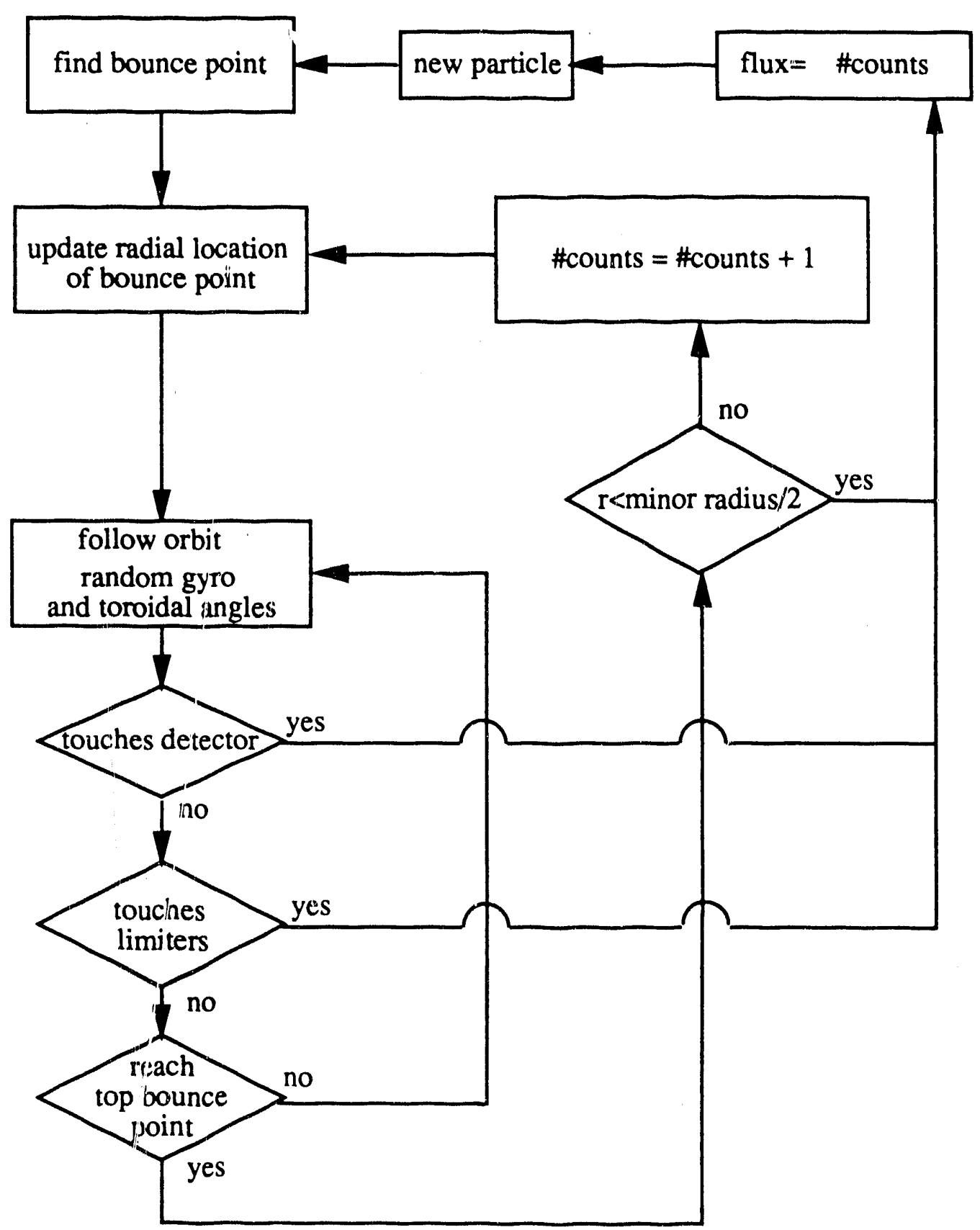

Figure A.5: Block diagram for the large obstacle ( $R F$ limiters) code. Compared to the small obstccle case a locp is added which follows the CFP orbit and checks for an impact on the limiters on the detector. 
which is the number of particles detected compared to the total CFPs produced. Here, $A$ is the total area of the slit, $T(\Omega)$ is the transmission coefficient of the collimating apertures, $S$ is the source profile function (see Eq. A.5), $d l$ is the length element along the particle orbit and $d l^{\prime}$ is a plasma volume element.

We checked the validity of this calculation by making a back-of-the-enveloppe calculation for a low plasma current case, in which all particles are simply drifting straight down $($ losses $=100 \%)$. We took detector \#6 which is located below the center of the vacuum vessel. The estimate of the efficiency is composed of three factors. The first one concerns the poloidal extension of the orbits viewed by the detector compared to the full poloidal area. In Fig. A.6 we show the poloidal projection of one orbit at very low current $(10 \mathrm{kA})$. The first factor is then:

$$
\int_{\text {poloidal }} \approx \frac{(2 \rho)\left(2 \int_{0}^{a} S(r) d r\right)}{4 \pi \int S(r) r d r} \approx 0.1
$$

The second factor is set by the range of gyroangles that can penetrate through the pinhole and slit which originate from the band shown in Fig. A.6 . By using the heights of the slit and the pinhole, and the distance between them (for an idea of the dimensions involved, see the example shown in Fig. 3.8) we find that the range is 0.17 radians. So only $0.17 / 2 \pi$ particles would be detected by the scintillator.

Finally, the third factor is simply set by the toroidal extension of the pinhole $(0.2 \mathrm{~cm})$ compared to the full length of the torus at that major radius $\left(=2 \pi R_{0}\right)$. Since the toroidal distribution of lost panticles at the bottom is uniform (axisymmetric), only $0.2 / 2 \pi R_{o}$ would be collected. Consequently, by grouping these factors one should recover the efficiency calculated by ORBIT.

$$
\begin{aligned}
& \text { efficiency } \approx \text { poloidal fraction } \times \text { gyroangle fraction } \times \text { toroidal fraction } \\
& 3.3 \times 10^{-7} \approx 0.1 \quad 0.025 \quad 1.3 \times 10^{-4}
\end{aligned}
$$

which agrees fairly well with the number obtained from ORBIT of $5.8 \times 10^{-7}$. 


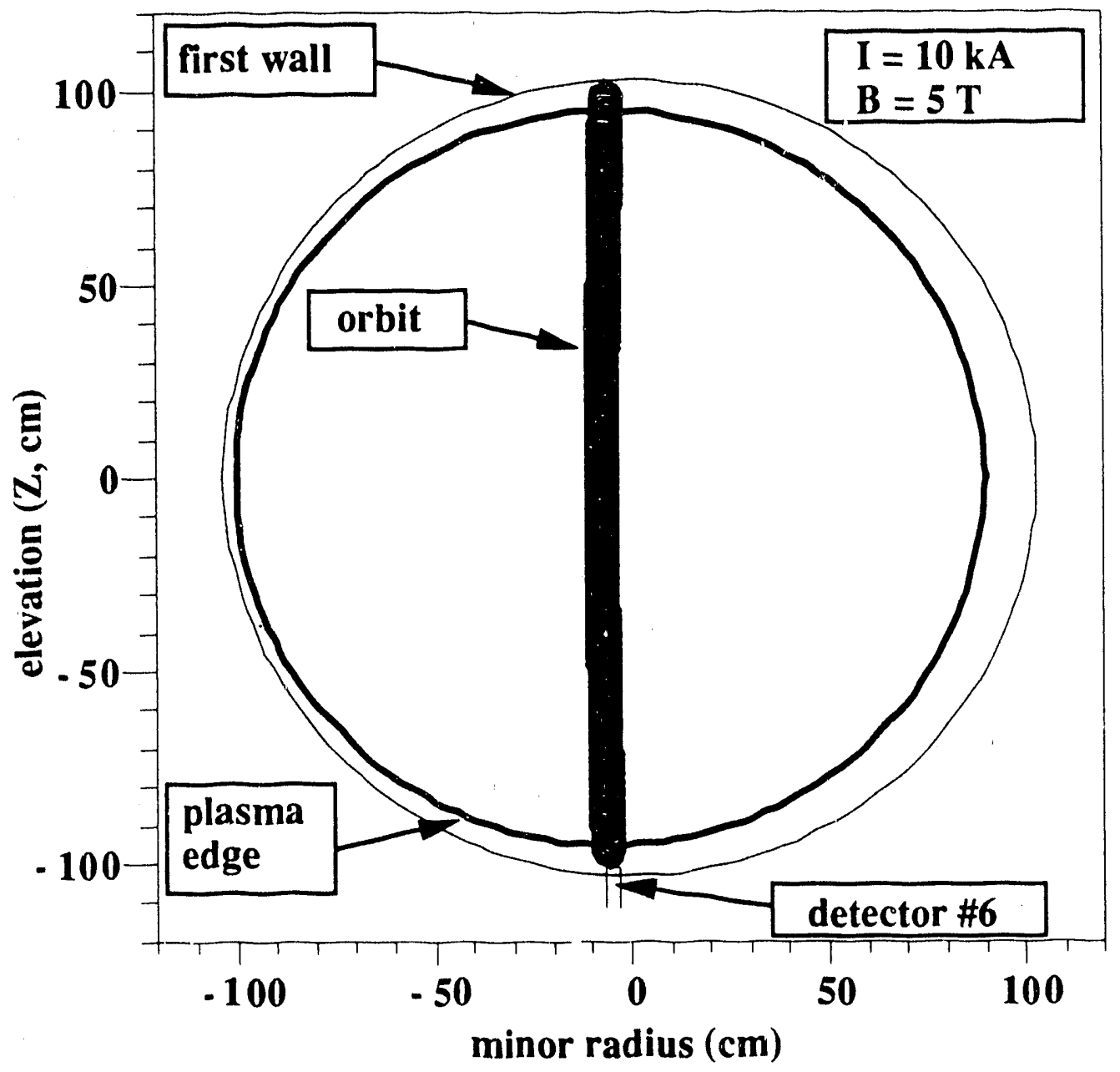

Figure A.6: Poloidal projection of an orbit reaching the bottom detector for a very low rurrent case $(10 \mathrm{kA})$. All CFPs are lost to the bottom of the vacuum vessel. The aperture can only "see" the region within 2 gyroradii directly above the detector. 


\section{Appendix B}

\section{Calibration details}

We describe in this appendix some of the details particular to our calibration setup and other elements of information germane to it.

\section{B.1 Calibration instrumentation}

The detectors used for the calibration of the $\mathrm{ZnS}(\mathrm{Ag})$ scintillator are of a readily available type. The Silicon Barrier Diode (SBD) (ORTEC model CR-21-300-300) is $300 \mu \mathrm{m}$ thick to allow full stopping of the 3 CFPs of interest and its surface is covered by a $0.2 \mu \mathrm{m}$ layer of aluminum. A negative bias of 90 volts was constantly applied to the SBD to allow full depletion of the detector. Dark current was found to disappear completely below -15 volts. When operating without the ion beam, we found that the noise level was very low; for example, with the alpha source $\left({ }^{241} \mathrm{Am}\right)$ the signal to noise ratio was better than 100 . However, in the shake-down phase, we had numerous problems with high frequency noise originating from the turbo pumps. As it was impossible to turn off the pumps during the run, we had to shes $d$ all the detector's components and use triax cables.

Signal from the SBD was sent to a preamplifier (model 142A) and at the last stage to an amplifier/single channel analyzer (Tennelec model TC 246). The output was directed to a counter (giving the number of protons, easy to discriminate) and to a Northern Tracor multi-channel analyzer model ECON II. A typical output from the multi-channel analyzer can be found in Fig. 3.18.

In parallel, we used, for looking at the scintillator, an Hamamatsu photomultiplier 
tube model R762 protected by a magnetic shield. The tube was always kept at a voltage of -800 volts; the gain was changed through the amplifier only, in order to avoid any non-linear regime of the tube. The signal was then sent to a Thorn EMI A-1 preamplifier/buffer set at maximum gain. The gain adjustment was made at the final step which was a delay line amplifier ORTEC model 460. The gain was changed only when we switched from the tritons + protons to the alpha source, in which case it was increased by a factor of 10 comparatively. The output was then sent to the multichannel analyzer described above.

\section{B.2 Beam parameters}

A Cockcroft-Walton type ion beam was used as the source of fast deuterons and, in the last stages of the calibration, could operate on a regular basis without breakdowns. The operating voltage was kept at $120 \mathrm{kV}$ and the beam current at approximately $200 \mu \mathrm{A}$ (measured by a Faraday cup). The accelerator has a set of magnets near the ion source for eliminating half and third energy components but they were removed in order to maximize the target loading of deuterjum. The beam focus was kept within a circle of less than $0.5 \mathrm{~cm}$ in diameter. We did not find that the heating of the target was a problem during the run since the fusion production did not decrease with time (in fact it increased slightly). The deuterium loading of the titanium target was done using the ion beam only. Since the shake-down phase of the detectors was spanned over many days, we estimated that the "full" target loading took several days (using only 10-20 $\mu \mathrm{A}$ of beam current for $\sim 8$ hours/day). Base pressure was usually below $10^{-6}$ torr in the target chamber except when using the alpha source, in which case it was kept at approximately 50 mtorr.

By examining the spectra from the SBD we found that in many cases half the fusion reactions were produced by the half energy component of the beam, as observed from the triton peak. The proton peak was found to be largely unaffected by the Doppler shift from the beam ions. The ${ }^{3}$ He component could be seen only with a very thin aluminum foil $(0.8 \mu \mathrm{m})$ where, with its small energy remaining, it had a negligible effect on the scintillator light output. Some runs were tried using no foil, in these cases the SBD was found to be "submerged" by scal ered beam ions and pile-up problems appeared at small pulse height.

The SBD was located approximately $9 \mathrm{~cm}$ from the target and recorded typically 
$10^{3}$ protons/sec through a circular mask (holding also the foil). Assuming an isotropic distribution of fusion products, we calculated that we produced between $10^{6}$ and $10^{7}$ fusion reactions per second. Although the sy'stem capabilities are certainly higher (at least on the point of view of beam current), we did not push the parameters in order to keep conditions the same during all the experiments and to prevent any spurious equipment failure.

\section{B.3 Particle stopping in materials}

One element that recurred many times in our discussion of the calibration was the deposition of energy by particles in a given material, namely aluminum or zinc sulfide. Directly connected is the particle range in those materials which we computed from tables edited by Ziegler et al. ${ }^{107,108}$ Since the stopping power $S \equiv d E / d x$ is a strong function of the particle energy $\left[S=S(E)_{j}\right.$, in order to know the particle range in a given material, one must integrate:

$$
\Delta x=\int_{E_{o}}^{E_{f}} \frac{d E}{S(E)}
$$

where $E_{f}$ and $E_{o}$ are respectively the final and initial energies. In Fig. 3.15 we showed the case of the full range of CFPs in $\mathrm{ZnS}$ (in this case $E_{f}=0$ ). We also needed to know the energy loss of CFPs through aluminum foils, especially through $3 \mu \mathrm{m}$. In Fig. B.1 we show the final energy of CFPs after a $3 \mu \mathrm{m} \mathrm{Al}$ foil as a function of their initial energy. We notice immediately that since this is a thin foil, the loss of energy is almost constant, showing only a small increase towards lower initial energies. This calculation was used to determinate the lower energy discriminats: of the detector (see Sect. 3.1.1).

In the case of $\mathrm{ZnS}$ we did not have the specific stopping power of the energetic ions. Instead we used a proper combination of the data from sulfur and zinc. We weighted the stopping contributions of each element by their molar weight according to the following:

$$
S_{Z_{n} S}=\frac{n_{Z_{n} S}}{M_{Z_{n} S}}\left(\frac{M_{S}}{n_{S}} S_{S}+\frac{M_{Z_{n}}}{n_{Z_{n}}} S_{Z_{n}}\right)
$$



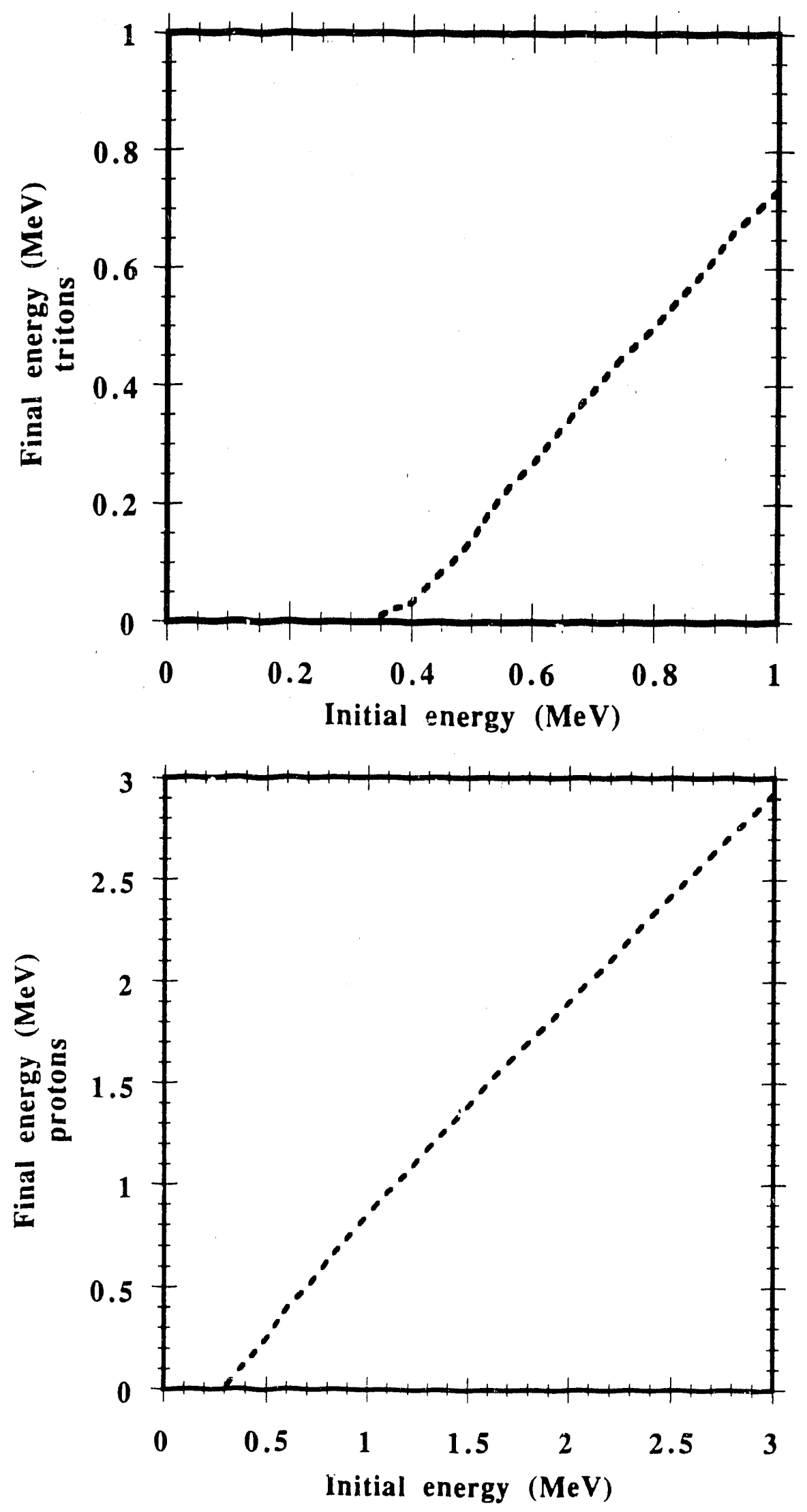


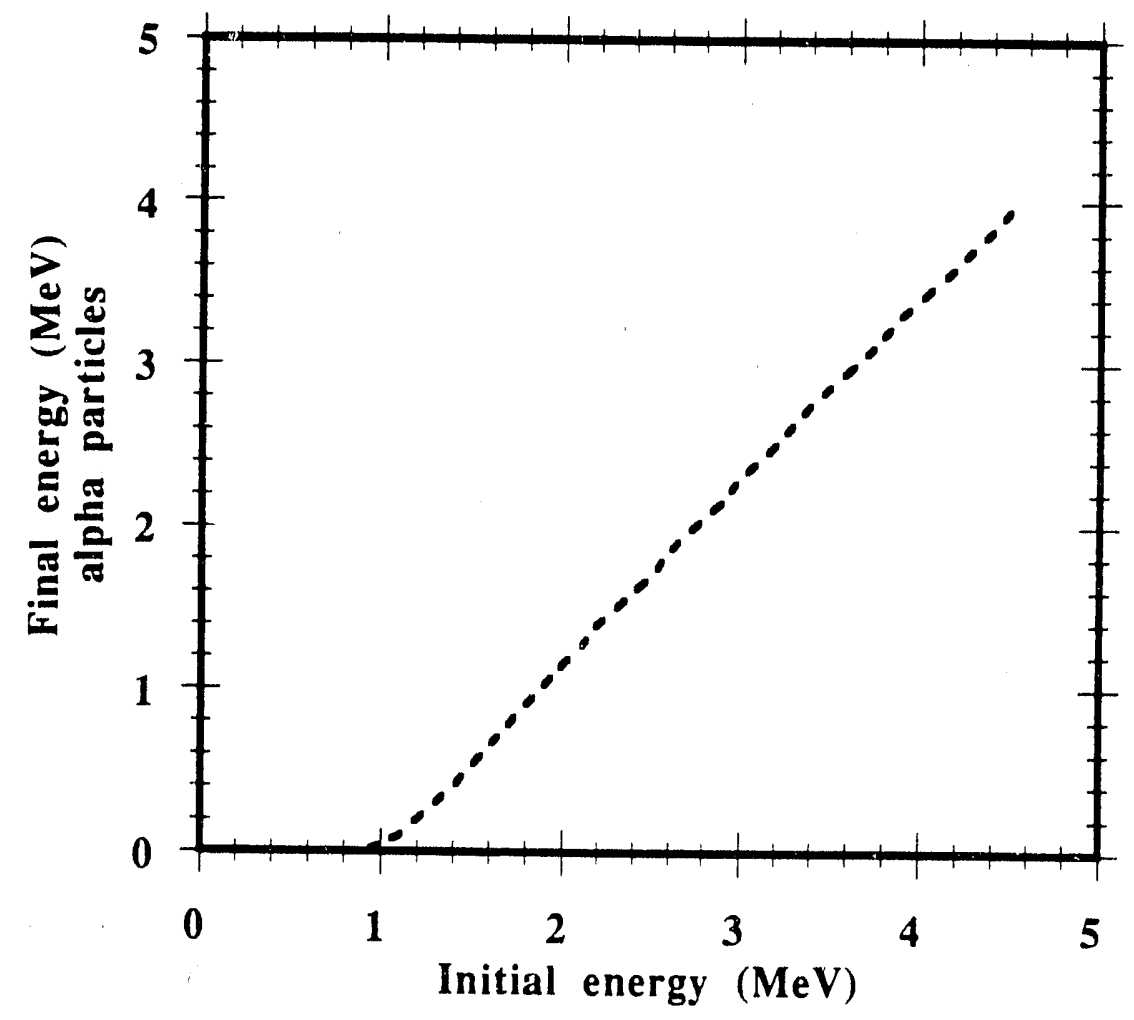

Figure B.1: Final energy of tritons (top), protons (middle), alphas (bottom) after passing through a $3 \mu \mathrm{m}$. Al foul as a function of the ir initial energy. 
where

$$
\begin{aligned}
& n_{Z n S}=4.1 \mathrm{mg} / \mathrm{cm}^{3} \text { density } \\
& n_{S}=2.1 \mathrm{mg} / \mathrm{cm}^{3} \\
& n_{Z n}=7.1 \mathrm{mg} / \mathrm{cm}^{3} \\
& M_{Z n S}=97 \mathrm{~g} / \text { mole molar weight } \\
& M_{S}=32 \mathrm{~g} / \text { mole } \\
& M_{Z n}=65 \mathrm{~g} / \text { mole } .
\end{aligned}
$$

\section{B.4 Other measurements}

The activity of the alpha source that we used $\left({ }^{241} \mathrm{Am}\right)$, was measured using the surface barrier detector. In vacuum, the $\mathrm{SBD}$ ) was positioned exactly one inch away from the source and connected to the same system described above. An aluminum mask was put in front of the detector in which a small hole was drilled through $(0.063$ " diameter). Assuming that the alpha source is isotropic (point source) we found that the activity of the source was $2.1 \times 10^{6} \pm 25 \%$ alphas $/ \mathrm{cm}^{2} / \mathrm{sec}$.

One critical aspect of our calibration was also the front to back ratio of the scintillator light emission. This measuremert. seeningly simple, was complicated by the need of having the photomultiplier tube close by the scintillator, which would block the alphas before they can reach the scintillator. Consequently we put the photomultiplier tube at $45^{\circ}$ with respect to the scintillator and at $90^{\circ}$ with respect to the stream of alphas (4.5 MeV). Shown in Fig. B.2 is the setup for both front and back measurements of the light emission. We used also a small mask (5/16" hole diameter) in order to restrict the area of particle impacts. The source was kept at the same distance (3") from the scintillator.

We also did some preliminary measurements of the light output from a P-31 $[\mathrm{ZnS}(\mathrm{Cu})]$ scintillator with approximately the same thickness and crystal grain size. We did the experiment with two different foils ( 2 and $18 \mu \mathrm{m}$ ) and we found that the P-31 scintillator is at least 5 times less efficient than the one we use, which is the P-11 $[\mathrm{ZnS}(\mathrm{Ag})]$. 
ERONT VIEW

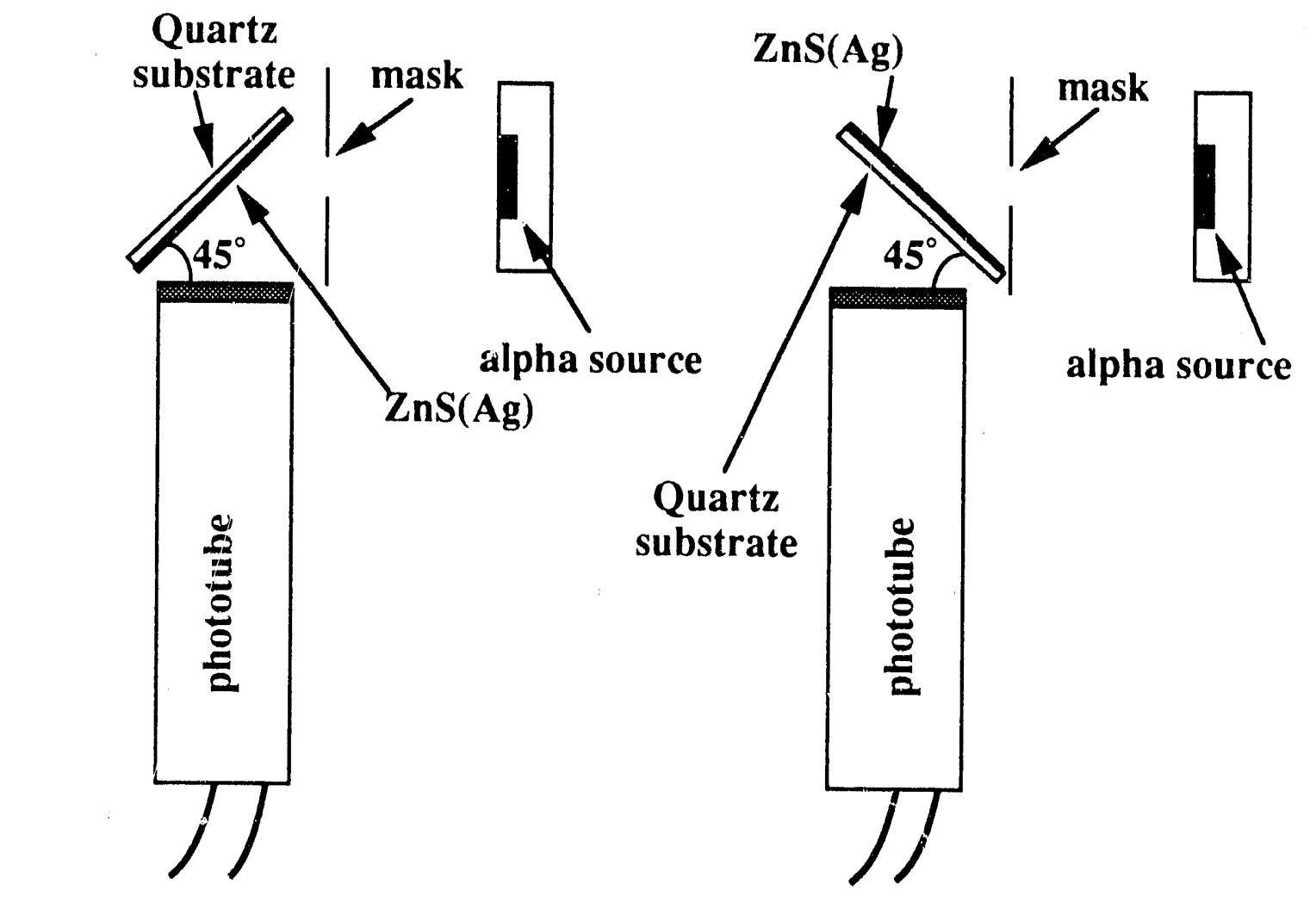

BACK VIEW

Figure B.2: Setup for front to back comparison of the light emission of the scintillator from $4.5 .1 \%$ I alphas. 


\section{Appendix $\mathbf{C}$}

\section{TFTR shot lists}

Shot list

Current scan large plasma $\left(R_{0}=2.60 \mathrm{~m}, \mathrm{~B}_{\mathrm{T}}=4 \mathrm{~T}\right)$

October $12^{\text {th }} 1990$

\begin{tabular}{lll} 
Shot number & plasma current (MA) & aperture position $(\mathrm{R}, \mathrm{cm})$ \\
\hline 55152 & 1.8 & 357.0 \\
55153 & 1.8 & 357.0 \\
55159 & 1.9 & 357.0 \\
55160 & 1.8 & 357.0 \\
55161 & 1.8 & 357.0 \\
55164 & 1.8 & 357.0 \\
55170 & 0.6 & 357.0 \\
55171 & 0.6 & 357.0 \\
55172 & 0.6 & 357.0 \\
55173 & 1.0 & 357.0 \\
55176 & 1.0 & 357.0 \\
55178 & 1.0 & 357.0 \\
55179 & 1.4 & 357.0 \\
55180 & 1.4 & 357.0 \\
55181 & 1.4 & 357.0 \\
55182 & 1.8 & 357.0 \\
55183 & 1.8 & 357.0 \\
55184 & 1.8 & 357.0
\end{tabular}

Table C.1: 
Shot list

Current scan "standard" plasma $\left(\mathrm{R}_{\mathrm{o}}=2.45 \mathrm{~m}, \mathrm{~B}_{\mathrm{T}}=5 \mathrm{~T}\right)$

September $22^{\text {nd }} 1990$

\begin{tabular}{|c|c|c|}
\hline Shot number & plasma current (MA) & aperture position $(\mathrm{R}, \mathrm{cm})$ \\
\hline 54441 & 1.6 & 354.5 \\
\hline 54446 & 1.6 & 354.5 \\
\hline 54447 & 1.6 & 347.5 \\
\hline 54449 & 1.6 & 347.5 \\
\hline 54450 & 1.6 & 347.5 \\
\hline 54451 & 1.6 & 347.5 \\
\hline 54452 & 1.6 & 347.5 \\
\hline 54453 & 1.6 & 354.5 \\
\hline 54454 & 0.8 & 354.5 \\
\hline 54455 & 0.8 & 347.5 \\
\hline 54456 & 0.8 & 347.5 \\
\hline 54457 & 0.8 & 347.5 \\
\hline 54458 & 0.8 & 347.5 \\
\hline 54459 & 0.8 & 347.5 \\
\hline 54460 & 0.8 & 347.5 \\
\hline 54461 & 1.2 & 354.5 \\
\hline 54462 & 1.2 & 347.5 \\
\hline 54463 & 1.2 & 347.5 \\
\hline 54464 & 1.8 & 354.5 \\
\hline 54466 & 1.8 & 347.5 \\
\hline 54467 & 1.8 & 347.5 \\
\hline 54468 & 2.0 & 354.5 \\
\hline 54469 & 2.0 & 347.5 \\
\hline 54470 & 2.0 & 347.5 \\
\hline 54471 & 2.0 & 347.5 \\
\hline 54472 & 2.0 & 354.5 \\
\hline 54474 & 1.4 & 354.5 \\
\hline 54475 & 1.4 & 347.5 \\
\hline 54476 & 1.4 & 347.5 \\
\hline 54481 & 1.4 & 338.5 \\
\hline 54482 & 1.4 & 343.5 \\
\hline 54157 & 1.1 & 354.5 \\
\hline 54176 & 1.1 & 347.5 \\
\hline 56247 & 0.6 & 3.54 .5 \\
\hline 56250 & 0.6 & 347.5 \\
\hline 56252 & 0.6 & 347.5 \\
\hline
\end{tabular}

Table C.2: 
Shot list

Radial and power scans small plasma $\left(R_{0}=2.25 \mathrm{~m}, \mathrm{~B}_{\mathrm{T}}=5 \mathrm{~T}, \mathrm{~L}_{\mathrm{p}}=1.15 \mathrm{MA}\right)$

September $15^{\text {th }} 1990$

\begin{tabular}{lll} 
shot number & aperture position $(\mathrm{R}, \mathrm{cm})$ & neutral beam power $(\mathrm{MW})$ \\
\hline 53920 & 350.1 & 5.0 \\
53921 & 349.1 & 7.5 \\
53922 & 347.0 & 5.2 \\
53923 & 345.0 & 7.5 \\
53924 & 343.0 & 10.4 \\
53925 & 341.0 & 7.7 \\
53926 & 341.0 & 7.7 \\
53927 & 343.0 & 7.7 \\
53929 & 343.0 & 2.7 \\
53930 & 343.0 & 5.3 \\
53931 & 343.0 & 7.8 \\
53932 & 343.0 & 2.7 \\
53933 & 343.0 & 7.7 \\
53934 & 343.0 & 10.0 \\
53935 & 343.0 & 10.0 \\
53936 & 343.0 & 10.0 \\
53937 & 343.0 & 10.0 \\
53938 & 343.0 & 10.0 \\
53939 & 343.0 & 10.0 \\
53940 & 343.0 & 10.0 \\
53941 & 343.0 & 10.0 \\
53945 & 343.0 & 10.0 \\
53946 & 343.0 & 10.0 \\
53947 & 348.0 & 7.7 \\
53948 & 340.0 & 7.7 \\
53949 & 346.0 & 7.7 \\
53950 & 342.0 & 7.7 \\
& &
\end{tabular}

Table C.3: 


\begin{tabular}{|c|c|c|}
\hline \multicolumn{3}{|c|}{$\begin{array}{l}\text { Shot list } \\
\text { Shadowing experiment (Bay A probe radial scan) } \\
\mathrm{I}_{\mathrm{p}}=1.4 \mathrm{MA}, \mathrm{B}_{\mathrm{T}}=5 \mathrm{~T}, \mathrm{R}_{\mathrm{o}}=2.45 \mathrm{~m} \\
\text { July 6th } 1990 \text {, September } 22 \text { nd } 1990\end{array}$} \\
\hline shot number & aperture position $(\mathrm{R}, \mathrm{cm})$ & probe $A$ position $(\mathrm{R}, \mathrm{cm})$ \\
\hline $\begin{array}{l}50203 \\
50204 \\
50209 \\
50210 \\
50211 \\
50212 \\
50214 \\
50215 \\
50216 \\
50217 \\
50218 \\
50219 \\
50220 \\
50221 \\
50223\end{array}$ & $\begin{array}{l}347.4 \\
347.4 \\
347.4 \\
347.4 \\
347.4 \\
347.4 \\
347.4 \\
347.4 \\
347.4 \\
347.4 \\
347.4 \\
347.4 \\
347.4 \\
347.4 \\
347.4\end{array}$ & $\begin{array}{l}370.0 \\
348.4 \\
363.4 \\
346.4 \\
344.4 \\
342.4 \\
354.4 \\
350.4 \\
340.4 \\
352.4 \\
338.4 \\
370.4 \\
338.4 \\
346.4 \\
342.4\end{array}$ \\
\hline $\begin{array}{l}54476 \\
54477 \\
54478 \\
54479 \\
54480\end{array}$ & $\begin{array}{l}347.4 \\
347.4 \\
347.4 \\
347.4 \\
347.4\end{array}$ & $\begin{array}{l}370.4 \\
349.4 \\
340.4 \\
330.4 \\
335.4\end{array}$ \\
\hline
\end{tabular}

Table C.4: 
Shot list

Detector radial scan $\left(\mathrm{I}_{\mathrm{p}}=1.6 \mathrm{MA}, \mathrm{R}_{\mathrm{o}}=2.45 \mathrm{~m}, \mathrm{~B}_{\mathrm{T}}=5 \mathrm{~T}\right)$

June $15^{\text {th }} 1990$

\begin{tabular}{ll} 
Shot Number & Aperture position $(\mathrm{cm})$ \\
\hline 49512 & 354.1 \\
49513 & 352.1 \\
49514 & 350.1 \\
49515 & 348.1 \\
49516 & 351.1 \\
49520 & 349.1 \\
49521 & 353.1 \\
49522 & 351.6 \\
49523 & 355.1 \\
49524 & 353.6 \\
49525 & 353.6 \\
49526 & 352.6 \\
49528 & 352.6 \\
49531 & 351.9 \\
49532 & 351.9 \\
49533 & 351.9 \\
49534 & 351.4 \\
49535 & 351.4 \\
49536 & 351.4 \\
49537 & 351.4 \\
49542 & 351.6 \\
49543 & 351.6 \\
49544 & 352.6 \\
49556 & 352.6 \\
49557 & 352.6 \\
49558 & 352.6 \\
49559 & 350.6 \\
& \\
\hline
\end{tabular}

Table C.5: 
Shot list

Complete detector radial scan $\left(\mathrm{I}_{\mathrm{p}}=1.15 \mathrm{MA}, \mathrm{R}_{\mathrm{o}}=2.45 \mathrm{~m}, \mathrm{~B}_{\mathrm{T}}=5 \mathrm{~T}\right)$

Sept. $19^{\text {th }} 1991$

\begin{tabular}{l|l} 
Shot number & Aperture position $(\mathrm{cm})$ \\
\hline 54173 & 351.50 \\
54174 & 350.50 \\
54175 & 349.50 \\
54176 & 347.50 \\
54177 & 345.50 \\
54178 & 343.50 \\
54179 & 341.50 \\
54180 & 338.50 \\
54181 & 339.50 \\
54182 & 342.50 \\
54183 & 344.50 \\
54184 & 340.50 \\
54185 & 346.50 \\
54186 & 350.50 \\
54187 & 352.00 \\
54188 & 354.00 \\
54189 & 354.00 \\
54190 & 354.50 \\
54191 & 355.50 \\
54192 & 355.00 \\
54193 & 355.00 \\
54194 & 353.00 \\
54195 & 348.50 \\
5496 & 348.50 \\
54197 & 352.50 \\
54198 & 353.50 \\
54199 & 353.50
\end{tabular}

Table C.6: 


\section{Bibliography}

[1] H. P. Furth, R. J. Goldston, S. J. Zweben, and D. J. Sigmar, Nucl. Fusion 30, $1799(1990)$.

[2. R. J. Goldston, R. B. White, and A. H. Boozer, Phys. Rev. Lett. 47, 647 (1!181).

(3) V. Y. Goloborod'ko and V. A. Yavorskij, Nucl. Fusion 29, 1025 (1989).

4. K. Tani, T. Takizuka, M. Azumi, and H. Kishimoto, Nucl. Fusion 23, 657 (1983).

5) F. Engelmann, Phys. Scr. T 16, 16 (1987).

6 D. E. Post et al., ITER physics, ITER Documentation Series No. 21, To be published by the IAEA, Vienna, 1991.

17. T. G. Northrop, editor, The Adiabatic Motion of Charged Particles, Interscience Publishers, New York, 1963.

8. R. B. White, Theory of Tokamak Plasmas, North-Holland, Amsterdam, 1989.

9) F. F. Chen. editor, Introduction to Plasma Physics and Controlled Fusion, Plenum Press, New York, 1984.

[10] R. J. Goldston, Fast Ion Diagnostic Experiment on ATC: Radially Resolved Measurements of $q, Z_{e f f}, T_{i \|}$ and $T_{i \downarrow}, \mathrm{PhD}$ thesis, Princeton University, Princeton, NJ, 1977.

[11] J. A. Rome and Y.-K. M. Peng, Nucl. Fusion 19, 1193 (1979).

[12] S. V. Putvinskii, Sov. J. Plasma Phys. 16, 634 (1990). 
13 S. I. Putvinskii, Sov. J. Plasma Phýs. 16, 735 (1990).

14] W. W. Heldbrink and J. D. Strachan, Rev. Sci. Instrum. 56, 501 (1985).

15! O. A. Anderson and H. P. Furth, Nucl. Fusion 12, 207 (1972).

16] T. E. Stringer, Nucl. Fusioii 12, 689 (1972).

17] J. N. Davidson, Nucl. Fusion 16, 731 (1976).

(18) R. J. Goldston and H. H. Towner, J. Plasma Phy's. 26, 283 (1981).

19! R. E. Duvall, Topics in Acton-Angle Methods Applied to Tokamak Transport and Multiphoton Excitation of Atomic Systems, PhD thesis, Princeton University, Princeton, NJ, 1990.

$20)$ P. Grua, Confinement des lons Suprathermiques dans un Tokamak Non Arisymmetrique, PhD thesis, Université Aix-Marseilles I, Provence, FRANC!E, 1989, also under report \#ELR-CEA-FC 1385.

21) P. Grua and J.-P. Roubin. Nucl. Fusion 30, 1499 (1990).

22. I). I. Brown. TFTK Magnetics Handbook, Technical Rep. PPPL-ID-2, Princeton P'lasma Physics Laboratory, 1983.

23) E. Bittoni and M. Haegi, Phys. Scr. T 16, 82 (1987).

24. E. Bittoni and M. Haegi, Fusion Tech. 18, 373 (1990).

25. L. M. Hively, Nucl. Fusion 24, 779 (1984).

26] L. M. Hively, Fusion Tech. 13, 438 (1988).

27 L. M. Hively and J. A. Rome, Nucl. Fusion 30, 1129 (1990).

28 K. B. White and H. E. Mynick, Physs. Fluids B 1, 980 (1989).

[29] H. E. Mỵnick and R. E. Duvall, Phỵs. Fluids B 1, 750 (1989).

30] S. V. Konovalov and S. V. Putvinskij, Fusion Tech. 18, 397 (1990).

31] M. Lisak, D. Andersón, H. Hammé, H. Wilhemsson, and M. Tendler, Nucl. Fusion 22, 515 (1982). 
[32] P. J. Catto and M. Tessarotto, Phys. Fluids 31, 2292 (1988).

[33] C. T. Hsu, P. J. Catto, and D. J. Sigmar, Phys. Fluids B 2, 280 (1990).

[34] S. J. Zweben, H. E. Mynick, R. Boivin, C. S. Chang, and G. Hammett, Slow Diffusion of Passing MeV lons in TFTR, Technical Report PPPL-2771, Princeton Plasma Physics Laboratory, 1991.

[35] D. Anderson and M. Lisak, Plasma Phys. Controlled Fusion 32, 141 (1990).

[36] G. J. Sadler et al., Fusion Tech. 18, 556 (1990).

[37] J. Källne et al., Nucl. Fusion 28, 1291 (1988).

[38] P. L. Colestock, J. D. Strachan, M. Ulrickson, and R. Chrien, Phys. Rev. Lett. 43, 768 (1979).

[39] F. E. Cecil, S. J. Zweben, and S. S. Medley, Nucl. Instrum. Methods 245, 547 (1986).

(40] U. Schumacher, Phys. Scr. T 16, 143 (1987).

[41] S. J. Zweb. n, Rev. Sci. Instrum. 57, 1723 (1986).

[42] S. J. Zweben, J. D. Strachan, and K. M. Young, Fusion Tech. 18, 573 (1990).

[43] J. D. Strachan, R. E. Chrien, and W. W. Heidbrink, J. Vac. Sci. Technol. A 1,811 (1983).

44] R. E. Chrien et al., Phys. Rev. Lett. 46, 535 (1981).

45] R. E. Chrien, Measurements of Fusion Reactions from a Tokamak Plasma, PhD thesis, Princeton University, Princeton, NJ, 1981.

46] K. E. Chrien, H. P. Eubank, D. M. Meade, and J. D. Strachan, Nucl. Fusion 21, 1661 (1981).

[47] R. E. Chrien and J. D. Strachan, Phys. Fluids 26, 1953 (1983).

[48] R. E. Chrien, R. Kaita, and J. D. Strachan, Nucl. Fusion 23, 1399 (1983). 
[49] W. W. Heidbrink, R. E. Chrien, and J. D. Strachan, Nucl. Fusion 23, 917 (1983).

$|50|$ W. W. Heidbrink, Nucl. Fusion 24, 636 (1984).

|51) W. W. Heidbrink, R. Hay, and J. D. Strachan, Phys. Rev. Lett. 53, 1905 (1984).

[52) W. W. Heidbrink, Tokamak Diagnostics Using Fusion Products, PhD thesis, Princeton University, Princeton, NJ, 1984.

53 W. W. Heidbrink, Nucl. Instrum. Methods 236, 380 (1985).

54 W. W. Heidbrink et al., Plasma Phys. Controlled Fusion 28, 871 (1986i).

55 W. W. Heidbrink, J. Lovberg, J. D. Strachan, and R. E. Bell, Nucl. Fusion 27, 129 (1987).

[56) J. Lovberg and J. D. Strachan, Nucl. Fusion 30, 2533 (1990).

557 T. J. Murphy and J. D. Strachan, Nucl. Fusion 25, 383 (1985).

158: S. Conroy, O. N. Jarvis, G. Sadler, and G. B. Huxtable, Nucl. Fusion 28, 2127 (1988).

159 (i. Martin et al., Phys. Scr. T 16, 171 (1987).

60) H. S. Bosch and U. Schumacher, Plasma Diagnostics with Charged Fusion Products from the D-D Reactions in ASDEX, in Basic and Advanced Diagnostics Techniques for Fusion Plasmas, Varenna, 1986, Vol 3, pages 761-766, Brussels, 1986, Commission of the European Communities.

61) H.-S. Bosch, Rev. Sci. Instrum. 61, 1699 (1990).

62 V. S. Zaveryaev et al, Sov. J. Plasma Phys. 16, 754 (1990).

63) G. Martin, P. Gilles, and P. Joyer, Fusion Profile Measurement on TORESUPRA, in Controlled Fusion and Plasma Phyizcs, (Proc. of the $17^{\text {th }}$ European Conf., Amsterdam, 1990), Vol 4, pages 1584 1587, Geneva, 1990, European Physical Society. 
[64] D. Hwang et al., High-Power ICRF and ICRF Plus Neutral-Beam Heating on PLT, in Plasma Physcis and Controlled Nuclear Fusion Research 1982, (Proc. of the $9^{\text {th }}$ Int. Conf., Baliimore, 1982), Vol. 2, pages 3-15, Vienna, 1983, IAEA.

[65] J. D. Strachan, Rev. Sci. Instrum. 57, 1771 (1986).

[66] G. Martin, Etude des Produits de Fusion Chargés dans un Tokamak, PhD thesis, Université de Paris-Sud, Paris, 1985, also under report\# EUR-CEA-FC-1273.

[67] J. D. Strachan and A. Chan, Nucl. Fusion 27, 1025 (1987).

[68] J. D. Strachan, Nucl. Fusion 29, 163 (1989).

[69] S. D. Scott et al., Local transport measurements during auxiliary heating in TFTR, paper IAEA-CN-53/A-3-6, IAEA Washington 1990.

170 R. Bastasz, D. Buchenauer, and S. J. Zweben, Rev. Sci. Instrum. 61, 3199 (1990).

171) E. V. Carruthers et al., J. Nucl. Mater. 176, 1027 (1990), Presented at the $9^{\text {th }}$ PSI Conference, Bournemouth, May 1990.

172 S. W. Conroy et al, Alpha-Particle Diagnostics for the D-T Phase, in Controlled Fusion and Plasma Physics,(Proc. of the $18^{\text {th }}$ European Conf., Berlin, 1991), Vol 4, pages 265-268, Geneva, 1991, European Physical Society.

173 A. Benninghoven et al., editors, Secondary Ion Mass Spectrometry SIMS VII, Wiley, Chicester, 1990.

[74] C. W. Magee, W. L. Harrington, and R. E. Honig, Rev. Sci. Instrum. 49, 477 (1978).

175 L. A. Artsimovich et al., Nucl. Fusion, 17 (1969), Special Supplement (Proc. Novosibirsk (ionf.).

176 W. Stodiek, K. Bol, H. Eubank, S. V. Goeler, and D. J. Grove, Effect of NonAxisymmetry Confining Fields on Tokamak Discharges, in Plasma Physics and Controlled Nuclear Fusion Research 1971, (Proc. of the $4^{\text {th }}$ Int. Conf., Madison, 1971), Vol. 1, pages 465-480, Vienna, 1971, IAEA. 
77) R. J. Goldston, S. Davis, and H. Eubank, Confinement Studies During Central Beam Injection in PLT, Technical Report PPPL-1733, Princeton Plasma Physics Laboratory, 1980.

78) S. Suckewer et al., Nucl. Fusion 21, 1301 (1981).

79] M. P. Petrov, Pis'ma Zh. Ehksp. Teor. Fiz. 17, 110 (1973).

80) V. V. Afromisov, E. L. Berez,rskii, A. B. Izvozchikov, and M. P. Petrov, Sov. J. Plasma Phys. 6, 133 (1980).

81] E. L. Berezovsky, S. L. Efremov, A. B. Izvozchikov, M. P. Petrov, and S. Y. Petrov, Peculiarities of the Ion Distribution Function in the T-10 Tokamak, in Controlled Fusion and Plasma Physics,(Proc. of the $10^{\text {th }}$ European Conf., Moscow,1981), Vol 1, pages 67-70, Geneva, 1981, European Physical Society.

82] E. L. Berezorskij et al., Nucl. Fusion 23, 1575 (1983).

83 S. D. Scott, An Experimental Investrgation of Magnetic Field Ripple Effects on Tokamak Plasmas, PhD thesis, Massachusetts Institute of Technology, Cambridge, MA, 1982.

84] S. D. Scott tt al., Nucl. Fusion 25, 359 (1985).

85 M. Greenwald, J. J. Schuss, and D. Cope, Nucl. Fusion 20, 783 (1980).

86) Equipe TFR, TFR Experiments on Superbanana Particle Diffusion, Small-Scale Turbulence and Transport, in Plasma Physcis and Controlled Nuclear Fusion Research 1980, (Proc. of the $8^{\text {th }}$ Int. Conf., Brussels, 1980), Vol. 1, pages 425437, Vienna, 1980, IAEA.

87. M. Tuszewski and J.-P. Roubin, Nucl. Fusion 28, 499 (1988).

88; J.-P. Roubin, Etude de l'Injection d'Atomes Neutres Rapides dans le Tokamak T.F.R., PhD thesis, Université de Paris-Sud, Paris, FRANCE, 1987, also under report \#FLR-CEA-FC-1331.

89| J. P. Roubin, D. Guilhem, G. Martin, B. Pégourié, and Y. Peysson, Observation of Strongly Localized Fast Particles Ripple Losses in TORE-SUPRA, in Controlled Fusion and Plasma Physics,(Proc. of the $18^{\text {th }}$ European Conf., Berlin, 1991), Vol 1, pages 53-56, Geneva, 1991, European Physical Society. 
[90] S. J. Zweben, Nucl. Fusion 29, 825 (1989).

(91) S. J. Zweben et al., Nucl. Fusion 30, 1551 (1990).

[92] S. J. Zweben, R. L. Boivin, C.S. Chang, G. Hammett, and H. E. Mynick, Search for Diffusion of Counter-Passing MeV Ions in the TFTR Tokamak, Technical Report PPPL-2770, Princeton Plasma. Physics Laboratory, 1991.

93 S. J. Zweben et al., Escaping $1 \mathrm{MeV}$ Tritons in TFTR, in Controlled Fusion and Plasma Physics,(Proc. of the $16^{\text {th }}$ European Conf., Venice, 1989), Vol 1, pages 39-42, Geneva, 1989, European Physical Society.

94) S. J. Zweben et al., Phys. Fluids B 2, 1411 (1990).

95] Z. Lin, private communication, (1991).

96] D. Curie, Luminescence in Crystals, John Wiley and Sons, New York, 1963.

[97] Phosphor Resource Manual for Industrial and Military Cathode Ray Tubes, published by the Imaging and Sensing Technology Corporation.

[98] S. J. Kilpatrick et al., J. Vac. Sci. Technol. A 4, 1817 (1986).

$99]$ R. Bastasz, S. J. Kilpatrick, and D. N. Ruzic, J. Nucl. Mater. 176, 1038 (1990), Presented at the $9^{\text {th }}$ PSI Conference, Bournemouth, May 1990.

$100\}$ R. L. Boivin, S. Kilpatrick, D. Manos, and S. J. Zweben, Rev. Sci. Instrum. $61,3208(1990)$.

[101\} J. Felt tet al., Rev. Sci. Instrum. 61, 3262 (1990).

102] F. A. Kröger, Some Aspects of the Luminescenct of Solids, Elsevier, Amsterdam. 1948.

$103]$ G. F. J. Garlick, Luminescent Matemuls, Clarendon Press, Oxford, 1949.

$104\} \mathrm{H}$. W. Leverenz, An Introduction to Luminescence of Solids, John Wiley and Sons, New York, 1950.

[105] J. B. Birks, The Theory and Practice of Scintillation Counting, Pergamon Press, Oxford, 1964. 
[106] G. F. Knoll, Radiation Detection and Measurement, John Wiley and Sons, New York, 1979.

[107| H. H. Andersen and J. F. Ziegler, Hydrogen Stopping Powers and Ranges in all Elemental Matter, volume 3, Pergarnon Press, New York, 1977.

[108] J. F. Ziegler, Helium Stopping Powers and Ranges in all Elemental Matter, volume 4, Pergamon Press, New York, 1977.

[109] P. Bayetti, F. Bottiglioni, G. Martin, and J. Paméla, Rev. Sci. Instrum. 57, 62 (1986).

[110] D. Markevich and R. R. Smith, Nucl. Instrum. Methods B 4, 388 (1984).

111] A. Stimson, Photometry and Radiometry for Engineers, John Wiley and Sons, New York, 1974.

[112] H. W. Hendel and D. L. Jassby, Nucl. Sci. and Eng. 106, 114 (1990), (and earlier references therein).

[113] A. L. Roquemore et al., Rev. Sci. Instrum. 61, 3163 (1990).

114) R. J. Hawryluk, An Empirical Approach to Tokamak Transport, in Physics of Plasmas Close to Thermonuclear Conditions, Varenna, 1979, Vol 1, pages 19-46, Brussels, 19\%0, Commission of the European Communities.

(115) M. ('. Zarnstorff et al., Convective Heat Transport in TFTR Supershots, in Controlled Fusion and Plasma Physics, (Proc. of the $15^{\text {th }}$ European Conf., Dubrovnik, 1988), Vol 1, pages 95-98, Geneva, 1988, European Physical Society.

$1116)$ E. Fredrickson, R. Colchin, K. McGuire, W. Morris, and N. Sauthoff, Rev. Sci. Instrum. 57, 2084 (1986).

[117] K. McGuire et al., Rev. Sci. Instrum. 57, 2136 (1986).

|118| K. B. White and M. S. Chance, Phys. Fluids 27, 2455 (1984).

[119] R. B. White, Phys. Fluids B 2, 845 (1990). 
[120 $\}$ J. J. Foit, K. Tani, T. Takizuka, and M. Azumi, Mapping Model for Trapped Particles in a Toroidal Ripple Field, Technical Report JAERI-M 88-249, Japan Atomic Energy Research Institute, 1988.

[121 V. Y. Goloborod'ko, Y. I. Kolesnichenko, V. V. Lutsenko, S. N. Resnik, and V. A. Yavorskij, Fusion Tech. 18, 429 (1990).

[122] W. Bauer, K. L. Wilson, C. L. Bisson, L. G. Haggmark, and R. J. Goldston, Nucl. Fusion 19, 93 (1979).

[123] V. S. Belikov, Y. I. Kolesnichenko, and V. A. Yavorskij, Nucl. Fusion 21, 1311 (1981).

124 V. Y. Goloborod'ko, Y. I. Kolesnichenko, and V. A. Yavorskij, Phys. Scr. T 16, 46 (1987).

125 K. Tani, Ripple Loss of Charged Fusion Products in TFTR and JT-60U, in Proc. of the First Workshop on Alpha Physics in TFTR, Princeton, $19^{\prime} 11$.

126 M. C. Zarnstorff et al., Advances in transport understanding using perturbative techniques in TFTR, paper IAEA-CN-53/A-2-2, IAEA Washington 1990.

127 N. N. Gorelenkov, Sov. J. Plasma Phys. 16, 241 (1990).

128 J. D. Strachan, Nucl. Fusion 16, 743 (1976).

129 D. Manos, R. V. Budny, S. Kilpatrick, P. Strangeby, and S. Zweben, Rev. Sci. Instrum. 57, $2107(1986)$.

130) D. M. Manos and S. J. Kilpatrick, Fast-Probe Plasma Diagnostics, in Process Dhagnostics: Materials, Combustion, Fusion (Proc. of the Mat. Res. Soc. Symp., Reno, 1988), Vol 117, edited by A. K. Hays, pages 263-274, Pittsburg, 1988, Materials Research Society.

131 W. W. Heidbrink et al., The Diffusion of Fast lons in Ohmic TFTR Discharges, Technical Report PPPL-2777, Princeton Plasma Physics Laboratory, 1991.

132 K. McGuire et al., Phys. Rev. Lett. 50, 891 (1983).

133| R. B. White et al., Phys. Fluids 26, 2958 (1983). 
(134) L. Chen and R. B. White, Phys. Rev. Lett. 52, 1122 (1984).

135] L. Chen et al., Theory and Simulation of Fishbone Type Instabilities in BeamHeated Tokarinaks, in Plasma Physcis and Controlled Nuclear Fusion Research 1994, (Proc. of the $10^{\text {th }}$ Int. Conf., London, 1982), Vol. 2, pages 59-67, Vienna, 1985, IAEA.

136] R. B. White, L. Chen, F. Romanelli, and R. Hay, Phys. Fluids 28, 278 (1985).

137 B. Coppi and F. Porcelli, Phys. Rev. Lett. 57, 2272 (1986).

138 K. Kaita et al. Phys. Fluids B 2, 1584 (1990).

139] S. J. Zweben, Rev. Sci. Instrum. 57, 1774 (1986).

$140 \mid$ D. J. Sigmar et al., 1991, Paper presented at the IAEA Technical Committee Neeting on Alpha Particles, Aspenas, SWEDEN.

141| S. Kilpatrick, private communication, (1991). 


\section{EXTERNAL DISTRIBUTION IN ADDITION TO UC-420}

Dr. F. Paoloni, Univ. of Wollongong, AUSTRALIA

Prof. M.H. Brennan, Univ. of Sydney, AUSTRALIA

Plasma Research Lab., Australian Nat Univ. AUSTRALIA

Prot. I.R. Jones. Flinders Univ, AUSTRALIA

Prol F. Cap, Inst for Theoretical Physics, AUSTRIA

Prot. M. Heindler, Institut für Theoretische Physik, AUSTRIA

Prot M Goossens, Astronomisch Instituut, BELGIUM

Ecole Royale Militaire, Lab. de Phy. Plasmas, BELGIUM

Commission-European, DG. XII-Fusion Prog., BELGIUM

Prof R. Boucique, Rijksuniversiteit Gent, BELGIUM

Mr PH. Sakanaka, Instituto Fisica, BRAZIL

instituto De Pesquisas Espaciais.INPE, BRAZIL

Documents OHtice, Atomic Energy of Canada Lid., CANADA

Dr. M.P. Bachynski, MPB Technologies, Inc., CANADA

Dr. H.M. Skarsgard, Univ. of Saskatchewan, CANADA

Prof J Teichmann, Univ. of Montreal, CANADA

Prot S R. Sreenivasan, Univ. of Calgary, CANADA

Prol. T.W. Johnston, INRS-Energie, CANADA

Dr $R$ Bolton, Centre canadien de fusion magnétique. CANADA

Dr. C.R James., Univ of Alberta, CANADA

Dr. P Lukac, Komenskeho Universzite, CZECHOSLOVAKIA

The Librarian, Culham Laboratory, ENGLAND

Library, R61. Ruttientord Appleton Laboratory, ENGLAND

Mis. S.A Hutchinson, JET Libran, ENGLAND

P Mahonen, Univ of Helsink, FINLAND

C Mouttel, Lab de Physique des Milieux lonisés, FRANCE

$\checkmark$ Radel. CEN/CADARACHE - Bat 506. FRANCE

MS C RInni. Univ of loannina. GREECE

Dr T Mual, Academy Biblıographic Ser. HONG KONG

Preprint Library. Hungarian Academy of Sci. HUNGARY

Dr B Das Gupta, Saha Inst of Nuclear Physics, INDIA

Di $P$ Kaw, Inst for Plasma Research, INDIA

Dr P Rosenau, Israel Inst of Technology. ISRAEL

Libranan, International Center for Theo Physics. ITALY

Miss C De Palo, Associazione EURATOM-ENEA, ITALY

Dr G Grosso, Istituto di Fisica del Plasma, ITALY

Dr $H$ Yamato. Toshiba Res \& Devel Center, JAPAN

Prof I Kawakami. Alomic Energy Res Inst., JAPAN

Prof K Nishikawa. Hiroshima Univ., JAPAN
Director, Japan Atomic Energy Research Inst., JAPAN

Prot. S. Itoh, Kyushu Univ., JAPAN

Data and Planning Center, Nagoya Univ, JAPAN

Prot. S. Tanaka, Kyoto Univ. JAPAN

Library, Kyolo Univ., JAPAN

Prot N. Inove, Univ of Tokyo, JAPAN

S. Mori, Technical Advisor, JAERI, JAPAN

O. Mitaral, Kumamoto Inst of Technology, JAPAN

$H$. Jeong, Korea Advanced Energy Research Insi. KOREA

Prot D.I. Chol. The Korea Adv Inst of Sci. \& Tech, KOREA

Prof. B.S. Liley, Univ. of Waikato, NEW ZEALAND

Inst. of Plasma Physics, PEOPLE'S REPUBLIC OF CHINA Libranan, Inst. of Physics, PEOPLE'S REPUBLIC OF CHINA Library, Tsinghua Univ., PEOPLE'S REPUBLIC OF CHINA

Z. Li, S.W. Inst Physics, PEOPLE'S REPUBLIC OF CHINA

Prot. J.A C Cabral, Instituto Superior Tecnico, PORTUGAL

Dr. O. Petrus, AL I CUZA Univ. ROMANIA

Dr. J. de Villiers, Fusion Studies, AEC, S AFRICA

Prot. M.A. Hellberg, Univ of Natal, S. AFRICA

C.I.EM.A.T, Fusion Division Library, SPAIN

Dr. L Stenflo, Univ. of UMEA, SWEDEN

Library, Royal Inst. of Technology, SWEDEN

Prof $H$ Wilhelmson. Chalmers Univ of Tech, SWEDEN

Centro Phys. Jes Plasmas, Ecole Polytech, SWITZERLAND

Bibliotheok, Inst Voor Plasma-Fysica. THE NETHERLANDS

M. Durgut, Viœ Chairman. Middle East Tech. Univ, TURKEY

Dr D D Ryutov, Sibenan Branch of Academy of Sci, USSR

Dr. GA Elıseev, Kurchatov Inst, USSR

Libranan. The UkJ SSR Academy of Sciences, USSR

Dr LM Kovrizhnykh, Inst of General Physics. USSR

Kerntorschungsanlage GmbH. Zentralbibliothek. W GERMANY

Bibliothek, Inst Fur Plasmatorschung. W GERMANY

Prof K Schındler, Ruhr-Universitál Bochum, W GERMANY

Dr F Wagner, (A.SDEX), Max-Planck-Instutut, W GERMANY

Libranan, Max-Planck-Instotut, W GERMANY

Prof RK Janev, Inst of Physics, YUGOSLAVIA 

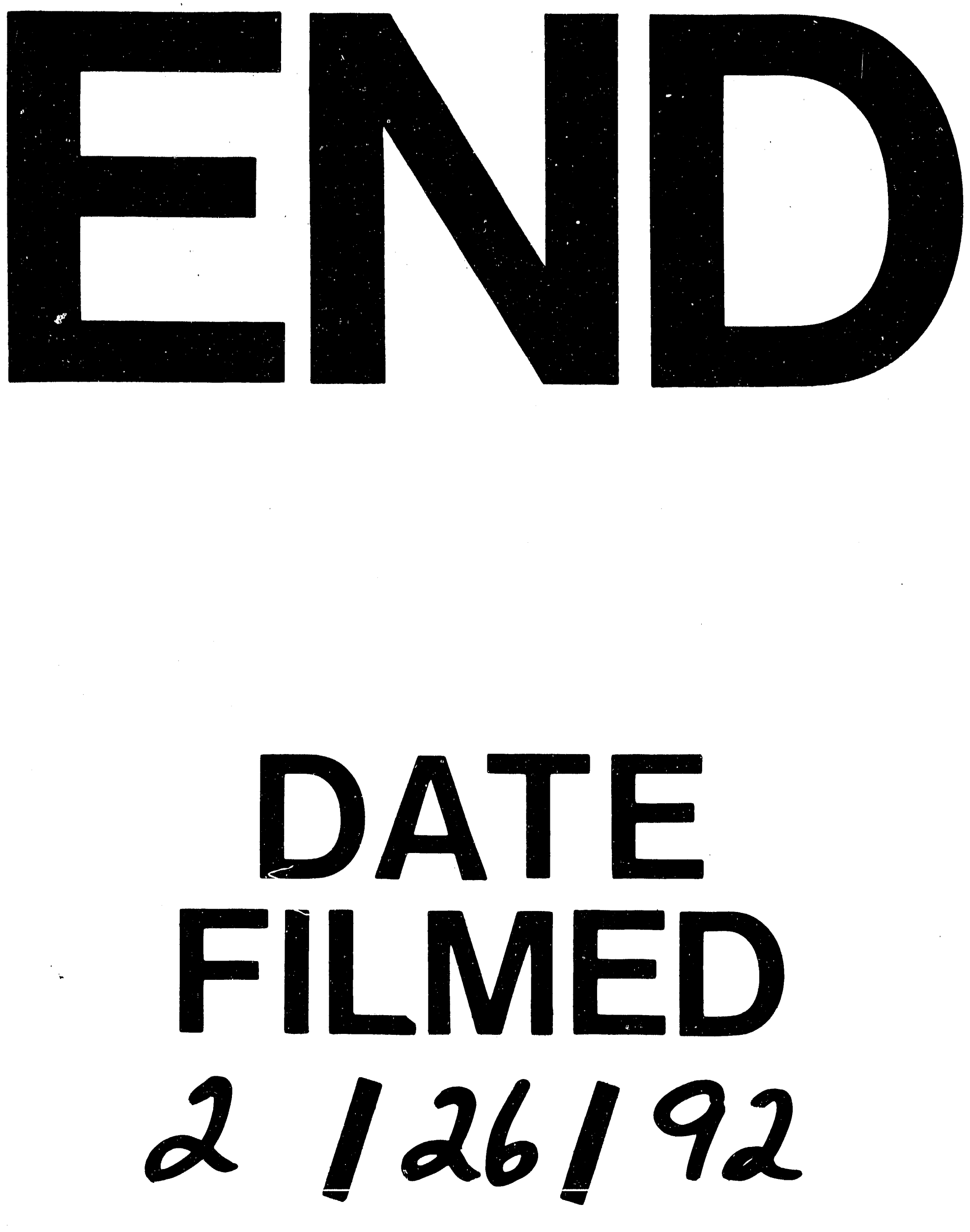
